\title{
Palladium-Catalyzed Carbonylative Cross-Coupling of Difluoroalkyl Halides with Alkylboranes under 1 atm of CO
}

Hai-Yang Zhao, ${ }^{\text {a, }}$ Minqi Zhou ${ }^{\mathrm{b}, *}$ and Xingang Zhanga,*

${ }^{a}$ Key Laboratory of Organofluorine Chemistry, Center for Excellence in Molecular Synthesis, Shanghai Institute of Organic Chemistry, University of Chinese Academy of Sciences, Chinese Academy of Sciences, 345 Lingling Lu, Shanghai 200032, China

${ }^{\mathrm{b} C o l l e g e}$ of Chemistry, Henan Institute of Advanced Technology Zhengzhou University, Zhengzhou 450001, China

${ }^{\ddagger}$. $-Y$. Zhao and M. Zhou contributed equally to this work. 


\section{Table of Contents}

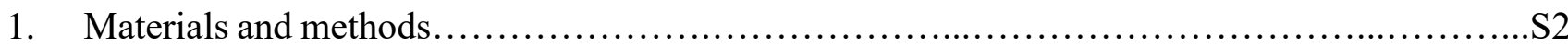

2. Preparation of alkyl-9-BBN reagents 1 and difluoroalkyl halides $2 \ldots \ldots \ldots \ldots \ldots \ldots \ldots \ldots \ldots . . . . . \ldots \ldots$

3. Optimization of the Pd-catalyzed carbonylation of alkyl-9-BBN 1 with difluoroalkyl halides 2

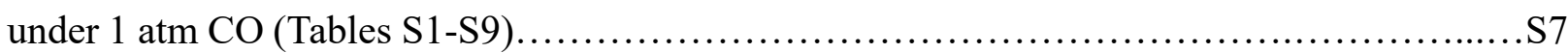

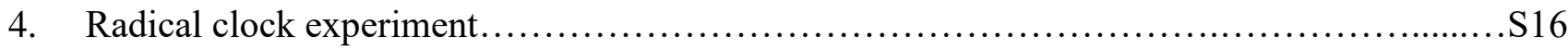

5. General procedure for the Pd-catalyzed carbonylation of alkyl-9-BBN 1 with difluoroalkyl

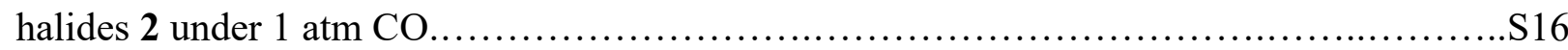

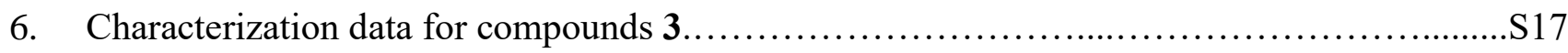

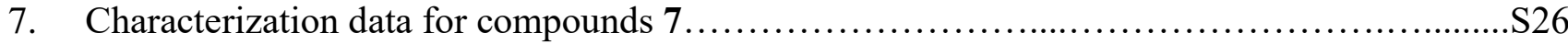

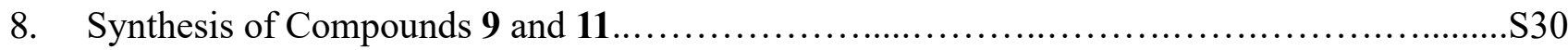

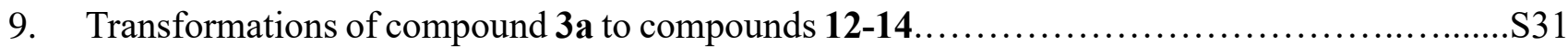

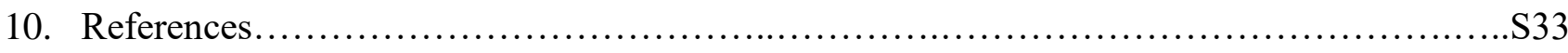

11. Copies of ${ }^{1} \mathrm{H}$ NMR, ${ }^{19} \mathrm{~F}$ NMR and ${ }^{13} \mathrm{C}$ NMR spectra of compounds 2-3, 7, 9, 11-14,

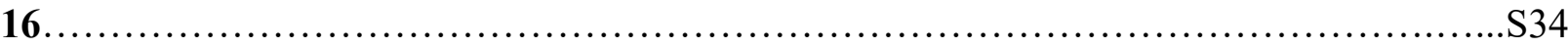




\section{Materials and methods}

General information: ${ }^{1} \mathrm{H}$ NMR and ${ }^{13} \mathrm{C}$ NMR spectra were recorded on an Agilent MR 400 and Agilent MR 500 spectrometer. ${ }^{19} \mathrm{~F}$ NMR was recorded on an Agilent MR 400 spectrometer $\left(\mathrm{CHCl}_{3}\right.$ at $7.26 \mathrm{ppm}{ }^{1} \mathrm{H}$ NMR; $\mathrm{CDCl}_{3}$ at $77.0 \mathrm{ppm}{ }^{13} \mathrm{C} \mathrm{NMR} ; \mathrm{CFCl}_{3}$ as an external standard and low field is positive ${ }^{19} \mathrm{~F}$ NMR). Chemical shifts $(\delta)$ are reported in ppm and coupling constants $(J)$ are in Hertz $(\mathrm{Hz})$. The following abbreviations were used to explain the multiplicities: $\mathrm{s}=$ singlet, $\mathrm{d}=$ doublet, $\mathrm{t}=$ triplet, $\mathrm{q}=$ quartet, $\mathrm{m}=$ multiplet, $\mathrm{br}=$ broad. $\mathrm{NMR}$ yield was determined by ${ }^{19} \mathrm{~F} \mathrm{NMR}$ using fluorobenzene as an internal standard before working up the reaction. HRMS measurements were recorded using EI with Waters Premier GC-TOF MS and DART with Thermo Fisher Scientific LTQ FTICR-MS.

Materials: All reagents were used as received from commercial sources or prepared as described in the literature. All reagents were handled under air and refilled with an inert atmosphere of Ar gas at room temperature. Dioxane was distilled from sodium immediately before use. alkyl-9-BBN reagents $\mathbf{1}$ were prepared according to the literature ${ }^{1}$. Compounds $\mathbf{2}$ were prepared according to the literature ${ }^{2-}$ 7 . 


\section{Preparation of alkyl-9-BBN reagents 1 and difluoroalkyl halides 2.}

2.1. General procedure for the preparation of alkyl-9-BBN reagents $1^{1}$ : In a glove box, the alkene $(10.0 \mathrm{mmol})$ was added to a suspension of 9-BBN dimer $(5.0 \mathrm{mmol})$ in dioxane $(8.0 \mathrm{~mL})$. The resulting mixture was stirred at $60^{\circ} \mathrm{C}$ for $30 \mathrm{~min}$ until the solution become clear. The mixture was then allowed to cool to room temperature, and dioxane was added to produce a total volume of $10 \mathrm{~mL}(1.0 \mathrm{M})$. The mixture could be used directly without further purification.

2.2. Preparation of difluoroalkyl halides 2: Compounds $2 \mathbf{a}-\mathbf{i},{ }^{2} \mathbf{2 j},{ }^{3} \mathbf{2 k},{ }^{4} \mathbf{2 m},{ }^{5} \mathbf{2 n},{ }^{6} \mathbf{2 o}{ }^{7}$ were prepared according to the literature. $\mathbf{2 g},{ }^{2} \mathbf{2} \mathbf{i},{ }^{2} \mathbf{2 j},{ }^{3} \mathbf{2 k},{ }^{4} \mathbf{2} \mathbf{m},{ }^{5} \mathbf{2 n},{ }^{6} \mathbf{2 o}{ }^{7}$ were known compounds. $\mathbf{2 l}$ is commercially available.<smiles>FC(F)(I)CCc1ccccc1</smiles>

$2 a$<smiles>FC(F)(I)CCCCCCl</smiles>

$2 e$

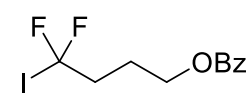

$2 \mathbf{i}$

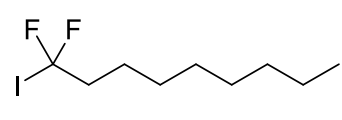

2b

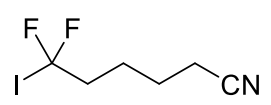

$2 f$

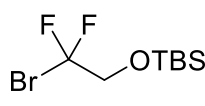

2j

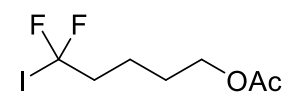

2c

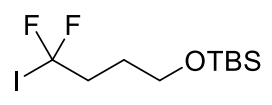

2d

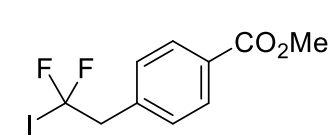

2g<smiles>O=C(OCCCC(F)(F)I)c1ccc(Cl)cc1</smiles>

$2 \mathrm{~h}$
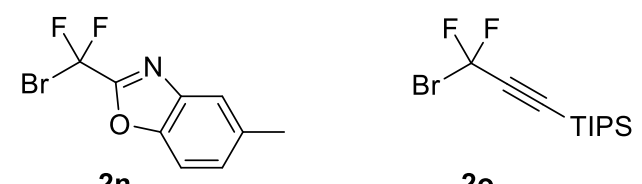

20 


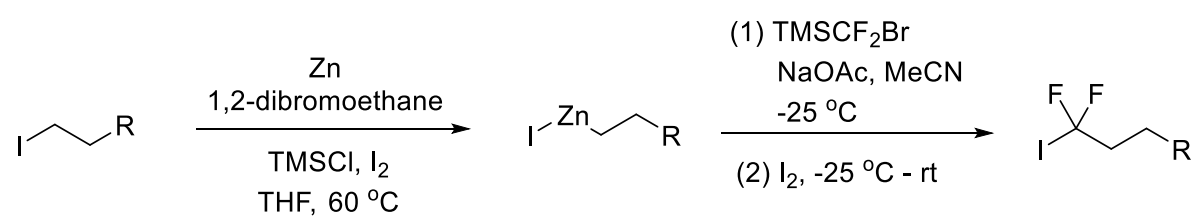

To a stirred suspension of zinc dust ( $45 \mathrm{mmol}, 1.5$ equiv) in THF (30 mL) was added one drop of 1,2dibromoethane. The mixture was stirred at $60^{\circ} \mathrm{C}$, then two drops of $\mathrm{Me}_{3} \mathrm{SiCl}$ were added. The resulting mixture was vigorously stirred for 15 minutes at $60{ }^{\circ} \mathrm{C}$. At this moment, the formation of gas was observed and zinc dust appeared as a dark-grey fuzzy material. Alkyl iodide (30 mmol, 1.0 equiv) was slowly added. After the resulting mixture was stirred at $60{ }^{\circ} \mathrm{C}$ for $18 \mathrm{~h}$, the unreacted zinc was allowed to settle down. The concentration of organozinc reagent was determined by iodometric titration.

A freshly titrated THF solution of organozinc reagent ( $5 \mathrm{mmol}, 1.0$ equiv) was concentrated under vacuum until the solid or viscous residue was formed. The residue was dissolved in freshly distilled $\mathrm{MeCN}(5 \mathrm{~mL})$. To the resulting solution was added sodium acetate (6 mmol, 1.2 equiv) at room temperature, the reaction flask was immersed in a cold bath at $-25{ }^{\circ} \mathrm{C}$, and the mixture was stirred for 10 minutes at $-25{ }^{\circ} \mathrm{C}$. Then, $\mathrm{Me}_{3} \mathrm{SiCF}_{2} \mathrm{Br}$ (6 mmol, 1.2 equiv) was added dropwise at $-25{ }^{\circ} \mathrm{C}$, and the reaction mixture was stirred at this temperature for $18 \mathrm{~h}$. Iodine $(5.25 \mathrm{mmol}, 1.05$ equiv) was added at $-25{ }^{\circ} \mathrm{C}$ to the reaction mixture with vigorous stirring. After dissolution of iodine, the cooling bath was removed, the mixture was allowed to warm to room temperature and was stirred for additional 5 hours. The resulting reddish-brown mixture was quenched by addition of aqueous $0.5 \mathrm{M}$ solution of $\mathrm{Na}_{2} \mathrm{~S}_{2} \mathrm{O}_{3}$ until decoloration, diluted with water, and extracted with PE. The combined organic layers were filtered through $\mathrm{Na}_{2} \mathrm{SO}_{4}$, concentrated under vacuum. The residue was purified by column chromatography on silica gel.

(3,3-Difluoro-3-iodopropyl)benzene (2a). The reaction was carried out on 9.8 mmol-scale from organozinc reagent. Compound $2 \mathbf{a}(2.4 \mathrm{~g}, 88 \%$ yield) as a colorless oil was purified with silica gel chromatography (Petroleum ether). ${ }^{1} \mathrm{H}$ NMR (400 MHz, $\left.\mathrm{CDCl}_{3}\right) \delta 7.35-7.18(\mathrm{~m}, 5 \mathrm{H}), 2.93-2.85$ $(\mathrm{m}, 2 \mathrm{H}), 2.71-2.57(\mathrm{~m}, 2 \mathrm{H}) .{ }^{19} \mathrm{~F}$ NMR $\left(376 \mathrm{MHz}, \mathrm{CDCl}_{3}\right) \delta-40.2(\mathrm{t}, J=14.7 \mathrm{~Hz}, 2 \mathrm{~F}) .{ }^{13} \mathrm{C}$ NMR $(101$ $\left.\mathrm{MHz} \mathrm{CDCl}_{3}\right) \delta 138.5,128.7,128.4,126.6,101.2(\mathrm{t}, J=315.4 \mathrm{~Hz}), 50.0(\mathrm{t}, J=19.5 \mathrm{~Hz}), 31.3(\mathrm{t}, J=$ 3.6 Hz). MS (EI): m/z (\%) $282(\mathrm{M})^{+}, 155,91(100), 77,65$. HRMS (EI) m/z: [M] ${ }^{+}$Calcd for $\mathrm{C}_{9} \mathrm{H}_{9} \mathrm{~F}_{2} \mathrm{I}$ : 281.9712; Found: 281.9713.

1,1-Difluoro-1-iodononane (2b). The reaction was carried out on $5 \mathrm{mmol}$-scale from organozinc reagent. Compound $\mathbf{2 b}$ (1.0 g, 72\% yield) as a colorless oil was purified with silica gel chromatography (Petroleum ether). ${ }^{1} \mathrm{H}$ NMR (400 MHz, $\left.\mathrm{CDCl}_{3}\right) \delta 2.35-2.22(\mathrm{~m}, 2 \mathrm{H}), 1.61-1.52(\mathrm{~m}, 2 \mathrm{H}), 1.42-$ 
$1.25(\mathrm{~m}, 10 \mathrm{H}), 0.89(\mathrm{t}, J=6.8 \mathrm{~Hz}, 3 \mathrm{H}) .{ }^{19} \mathrm{~F} \mathrm{NMR}\left(376 \mathrm{MHz}, \mathrm{CDCl}_{3}\right) \delta-35.2(\mathrm{t}, J=15.0 \mathrm{~Hz}, 2 \mathrm{~F}) .{ }^{13} \mathrm{C}$ NMR $\left(101 \mathrm{MHz}, \mathrm{CDCl}_{3}\right) \delta 102.8(\mathrm{t}, J=315.0 \mathrm{~Hz}), 48.2(\mathrm{t}, J=19.2 \mathrm{~Hz}), 31.8,29.2,29.1,28.3,25.2$ $(\mathrm{t}, J=3.2 \mathrm{~Hz}), 22.6,14.1$. MS (EI): m/z (\%) $270(\mathrm{M}-\mathrm{HF})^{+}, 121,107,81,57$ (100). HRMS (FI) m/z: $[\mathrm{M}-\mathrm{H}]^{+}$Calcd for $\mathrm{C}_{9} \mathrm{H}_{16} \mathrm{~F}_{2} \mathrm{I}: 289.0259$; Found: 289.0253 .

5,5-Difluoro-5-iodopentyl acetate (2c). The reaction was carried out on 5 mmol-scale from organozinc reagent. Compound $2 \mathrm{c}(1.5 \mathrm{~g}, 78 \%$ yield $)$ as a colorless oil was purified with silica gel chromatography (Petroleum ether: Ethyl acetate $=50: 1) .{ }^{1} \mathrm{H} \mathrm{NMR}\left(400 \mathrm{MHz}, \mathrm{CDCl}_{3}\right) \delta 4.08(\mathrm{t}, J=$ $6.4 \mathrm{~Hz}, 2 \mathrm{H}), 2.40-2.27(\mathrm{~m}, 2 \mathrm{H}), 2.05(\mathrm{~s}, 3 \mathrm{H}), 1.76-1.59(\mathrm{~m}, 4 \mathrm{H}) .{ }^{19} \mathrm{~F}$ NMR $\left(376 \mathrm{MHz}, \mathrm{CDCl}_{3}\right) \delta$ $35.9(\mathrm{t}, J=15.0 \mathrm{~Hz}, 2 \mathrm{~F}) .{ }^{13} \mathrm{C} \mathrm{NMR}\left(126 \mathrm{MHz}, \mathrm{CDCl}_{3}\right) \delta 171.1,101.9(\mathrm{t}, J=313.7 \mathrm{~Hz}), 63.6,47.6(\mathrm{t}$, $J=19.7 \mathrm{~Hz}), 27.1,21.9(\mathrm{t}, J=3.2 \mathrm{~Hz}), 20.9$. MS (EI): m/z (\%) $293(\mathrm{M}+\mathrm{H})^{+}, 185,127,105,77(100)$. HRMS (FI) m/z: [M-H] ${ }^{+}$Calcd for $\mathrm{C}_{7} \mathrm{H}_{11} \mathrm{O}_{2} \mathrm{~F}_{2} \mathrm{I}$ : 291.9766; Found: 291.9770.

tert-Butyl(4,4-difluoro-4-iodobutoxy)dimethylsilane (2d). The reaction was carried out on 5 mmolscale from organozinc reagent. Compound $2 \mathrm{~d}(1.1 \mathrm{~g}, 65 \%$ yield) as a colorless oil was purified with silica gel chromatography (Petroleum ether). ${ }^{1} \mathrm{H} \mathrm{NMR}\left(400 \mathrm{MHz}, \mathrm{CDCl}_{3}\right) \delta 3.68(\mathrm{t}, J=6.0 \mathrm{~Hz}, 2 \mathrm{H})$, $2.47-2.34(\mathrm{~m}, 2 \mathrm{H}), 1.82-1.73(\mathrm{~m}, 2 \mathrm{H}), 0.90(\mathrm{~s}, 9 \mathrm{H}),-0.06(\mathrm{~s}, 6 \mathrm{H}) .{ }^{19} \mathrm{~F} \mathrm{NMR}\left(376 \mathrm{MHz}, \mathrm{CDCl}_{3}\right) \delta-$ $35.2(\mathrm{t}, J=15.4 \mathrm{~Hz}, 2 \mathrm{~F}) .{ }^{13} \mathrm{C} \mathrm{NMR}\left(101 \mathrm{MHz}, \mathrm{CDCl}_{3}\right) \delta 102.5(\mathrm{t}, J=314.5 \mathrm{~Hz}), 61.0,45.1(\mathrm{t}, J=19.7$ $\mathrm{Hz}), 28.6(\mathrm{t}, J=3.1 \mathrm{~Hz}), 25.9,18.3$, -5.37. MS (EI): m/z (\%) $349(\mathrm{M}-\mathrm{H})^{+}, 199$ (100), 169, 155, 89, 72. HRMS (FI) m/z: [M+H $]^{+}$Calcd for $\mathrm{C}_{10} \mathrm{H}_{22} \mathrm{OF}_{2} \mathrm{ISi}$ : 351.0447; Found: 351.0452.

6-Chloro-1,1-difluoro-1-iodohexane (2e). The reaction was carried out on 5 mmol-scale from organozinc reagent. Compound $2 \mathrm{e}(1.2 \mathrm{~g}, 56 \%$ yield) as a yellow oil was purified with silica gel chromatography (Petroleum ether). This compound (64\%) was mixed with 1-chloro-5-iodopentane. ${ }^{1} \mathrm{H}$ NMR $\left(400 \mathrm{MHz}, \mathrm{CDCl}_{3}\right) \delta 3.55(\mathrm{t}, J=6.8 \mathrm{~Hz}, 2 \mathrm{H}), 2.39-2.26(\mathrm{~m}, 2 \mathrm{H}), 1.65-1.49(\mathrm{~m}, 6 \mathrm{H}) .{ }^{19} \mathrm{~F}$ NMR $\left(376 \mathrm{MHz}, \mathrm{CDCl}_{3}\right) \delta-35.6(\mathrm{t}, J=15.0 \mathrm{~Hz}, 2 \mathrm{~F}) .{ }^{13} \mathrm{C} \mathrm{NMR}\left(126 \mathrm{MHz}, \mathrm{CDCl}_{3}\right) \delta 102.1(\mathrm{t}, J=$ $313.7 \mathrm{~Hz}), 47.9$ (t, $J=19.7 \mathrm{~Hz}), 44.6,32.1,25.5,24.6(\mathrm{t}, J=3.3 \mathrm{~Hz}) . \mathrm{MS}(\mathrm{EI}): \mathrm{m} / \mathrm{z}(\%) 262(\mathrm{M}-\mathrm{HF})^{+}$, 155, 135, 127, 119 (100), 99. HRMS (FI) m/z: [M] ${ }^{+}$Calcd for $\mathrm{C}_{6} \mathrm{H}_{10} \mathrm{ClF}_{2} \mathrm{I}: 281.9478$; Found: 281.9482.

6,6-Difluoro-6-iodohexanenitrile (2f). The reaction was carried out on $5 \mathrm{mmol}$-scale from organozinc reagent. Compound $\mathbf{2 f}(1.0 \mathrm{~g}, 80 \%$ yield $)$ as a colorless oil was purified with silica gel chromatography (Petroleum ether). ${ }^{1} \mathrm{H}$ NMR (400 MHz, $\left.\mathrm{CDCl}_{3}\right) \delta 2.42-2.29(\mathrm{~m}, 4 \mathrm{H}), 1.80-1.67(\mathrm{~m}, 4 \mathrm{H}) .{ }^{19} \mathrm{~F} \mathrm{NMR}$ $\left(376 \mathrm{MHz}, \mathrm{CDCl}_{3}\right) \delta-36.1(\mathrm{t}, J=14.7 \mathrm{~Hz}, 2 \mathrm{~F}) .{ }^{13} \mathrm{C} \mathrm{NMR}\left(101 \mathrm{MHz}, \mathrm{CDCl}_{3}\right) \delta 118.9,101.0(\mathrm{t}, J=$ 
314.4 Hz), $47.1(\mathrm{t}, J=20.0 \mathrm{~Hz}), 24.3(\mathrm{t}, J=3.4 \mathrm{~Hz}), 23.9,16.9 . \mathrm{MS}(\mathrm{EI}): \mathrm{m} / \mathrm{z}(\%) 259(\mathrm{M})^{+}, 132(100)$, 127, 112, 85, 77. HRMS (FI) m/z: [M] ${ }^{+}$Calcd for $\mathrm{C}_{6} \mathrm{H}_{8} \mathrm{NF}_{2} \mathrm{I}: 258.9664$; Found: 258.9661.

4,4-Difluoro-4-iodobutyl 4-chlorobenzoate $(2 \mathrm{~h})$. The reaction was carried out on $5 \mathrm{mmol}$-scale from organozinc reagent. Compound $2 \mathrm{c}(1.3 \mathrm{~g}, 67 \%$ yield) as a yellow oil was purified with silica gel chromatography (Petroleum ether: Ethyl acetate = $100: 1) .{ }^{1} \mathrm{H}$ NMR $\left(400 \mathrm{MHz}, \mathrm{CDCl}_{3}\right) \delta 7.95(\mathrm{~d}, J=$ $7.6 \mathrm{~Hz}, 2 \mathrm{H}), 7.40$ (d, $J=7.6 \mathrm{~Hz}, 2 \mathrm{H}), 4.37$ (t, $J=5.2 \mathrm{~Hz}, 2 \mathrm{H}), 2.57-2.42(\mathrm{~m}, 2 \mathrm{H}), 2.10-2.00(\mathrm{~m}$, 2H). ${ }^{19} \mathrm{~F} \mathrm{NMR}\left(376 \mathrm{MHz}, \mathrm{CDCl}_{3}\right) \delta-36.4(\mathrm{t}, J=15.0 \mathrm{~Hz}, 2 \mathrm{~F}) .{ }^{13} \mathrm{C} \mathrm{NMR}\left(101 \mathrm{MHz}, \mathrm{CDCl}_{3}\right) \delta 165.4$, 139.6, 130.9, 128.8, 128.3, 100.9 (t, $J=313.8 \mathrm{~Hz}), 63.0,45.0(\mathrm{t}, J=20.4 \mathrm{~Hz}), 24.3(\mathrm{t}, J=3.5 \mathrm{~Hz})$. MS (EI): m/z (\%) $373(\mathrm{M})^{+}, 247,156,139$ (100), 111, 91. HRMS (FI) m/z: [M] ${ }^{+}$Calcd for $\mathrm{C}_{11} \mathrm{H}_{10} \mathrm{O}_{2} \mathrm{ClF}_{2} \mathrm{I}$ : 373.9377; Found: 373.9383. 
3. Optimization of the Pd-catalyzed carbonylation of alkyl-9-BBN 1 with difluoroalkyl halides 2 under 1 atm CO (Tables S1-S9).

Table S1. Screening of the palladium sources. ${ }^{a}$

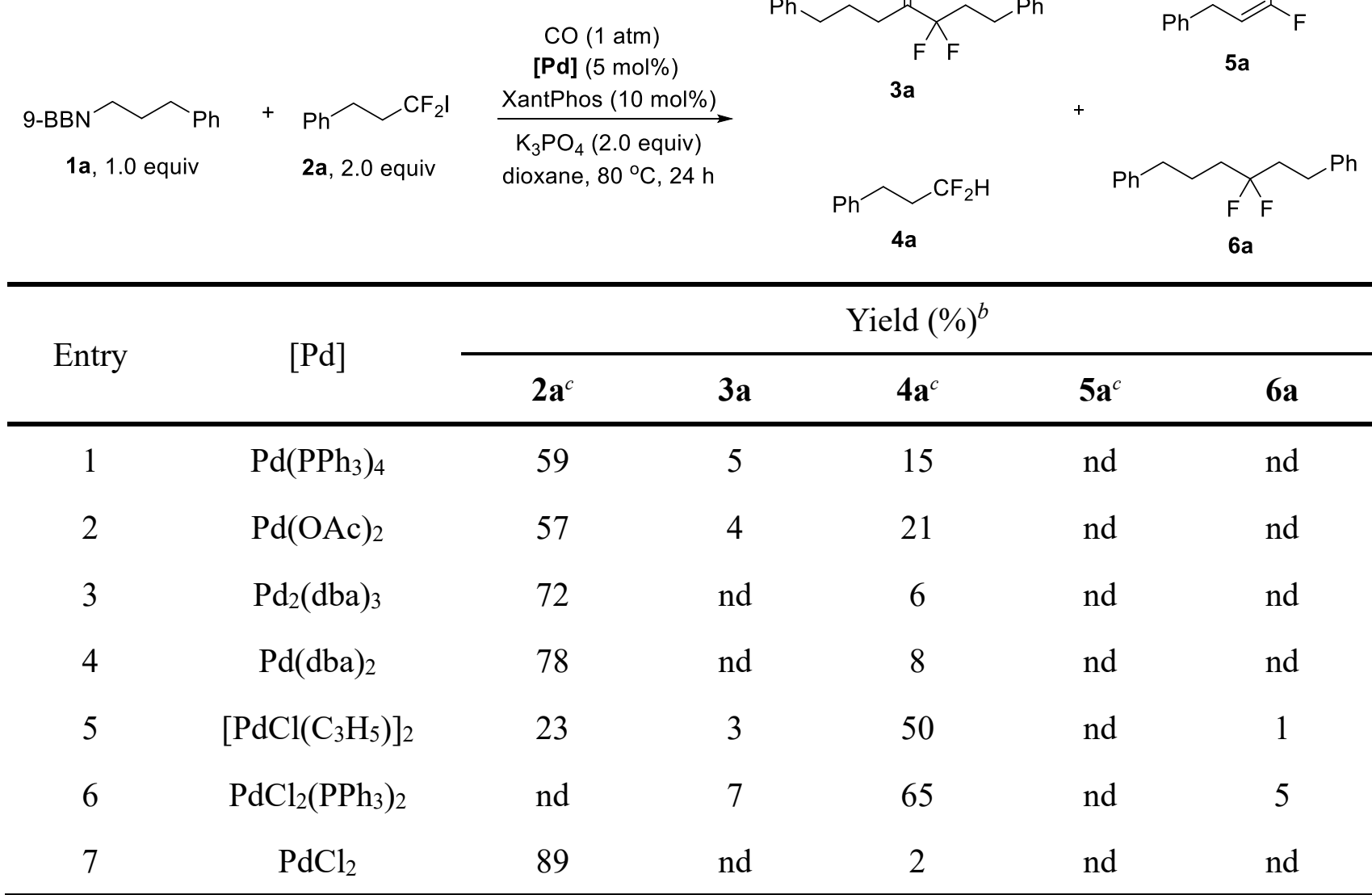

${ }^{a}$ Reaction conditions: $1 \mathbf{a}\left(0.4 \mathrm{mmol}, 1.0\right.$ equiv), $\mathbf{2 a}\left(0.8 \mathrm{mmol}, 2.0\right.$ equiv), dioxane $(2.0 \mathrm{~mL}), 24 \mathrm{~h}, 80{ }^{\circ} \mathrm{C} .{ }^{b}$ Determined by ${ }^{19} \mathrm{~F}$ NMR using fluorobenzene as the internal standard; nd, not detected. ${ }^{c}$ The yields of $\mathbf{2 a}, \mathbf{4 a}, \mathbf{5 a}$ were calculated based on $\mathbf{2 a}$. 
Table S2. Screening of the reaction temperature. ${ }^{a}$

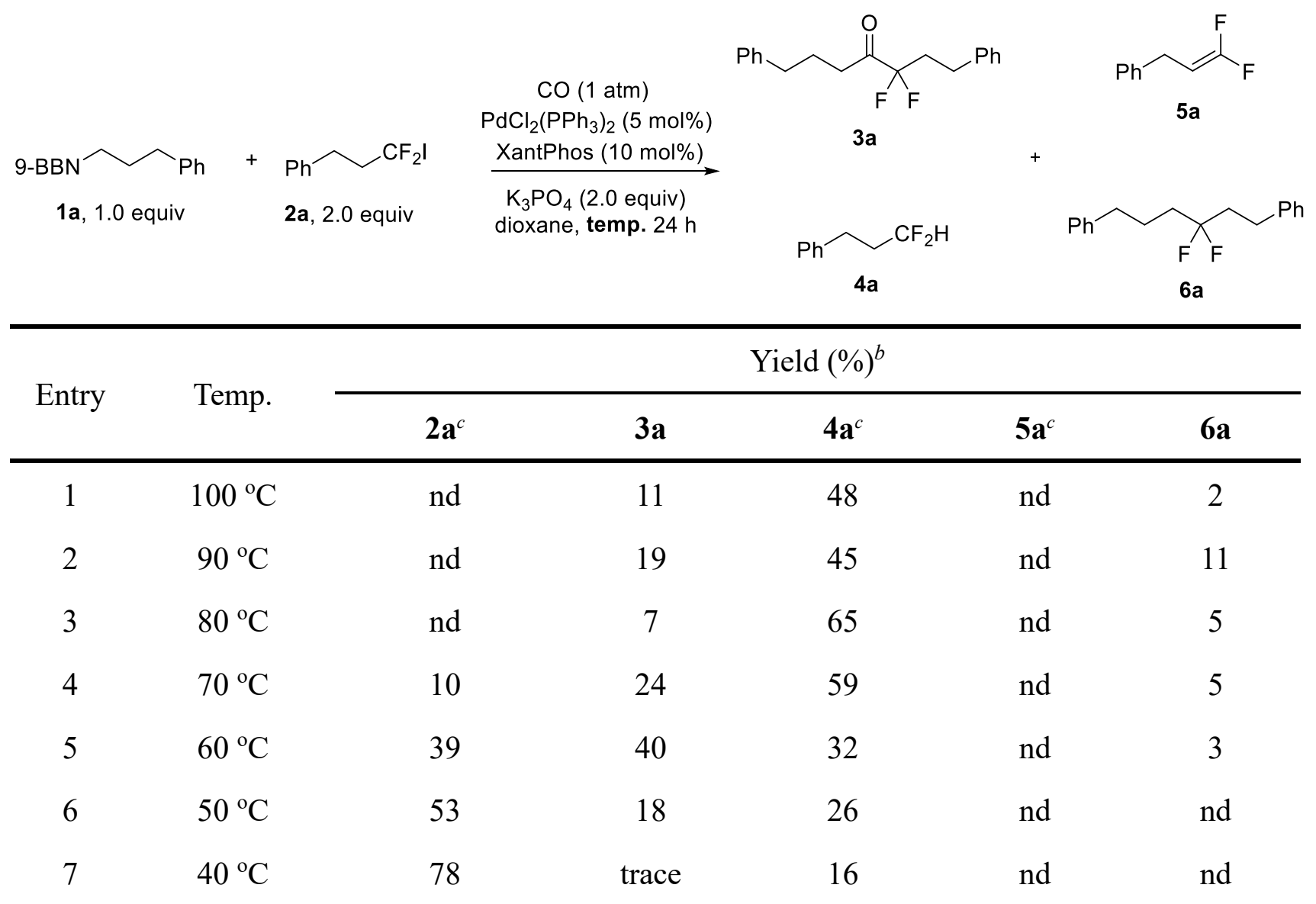

${ }^{a}$ Reaction conditions: 1a $\left(0.4 \mathrm{mmol}, 1.0\right.$ equiv), 2a $\left(0.8 \mathrm{mmol}, 2.0\right.$ equiv), dioxane $(2.0 \mathrm{~mL}), 24 \mathrm{~h} .{ }^{b}$ Determined by

${ }^{19} \mathrm{~F}$ NMR using fluorobenzene as the internal standard; nd, not detected. ${ }^{\mathrm{C}}$ The yields of $\mathbf{2 a}, \mathbf{4 a}, \mathbf{5 a}$ were calculated based on $\mathbf{2 a}$. 
Table S3. Screening of the ligands. ${ }^{a}$

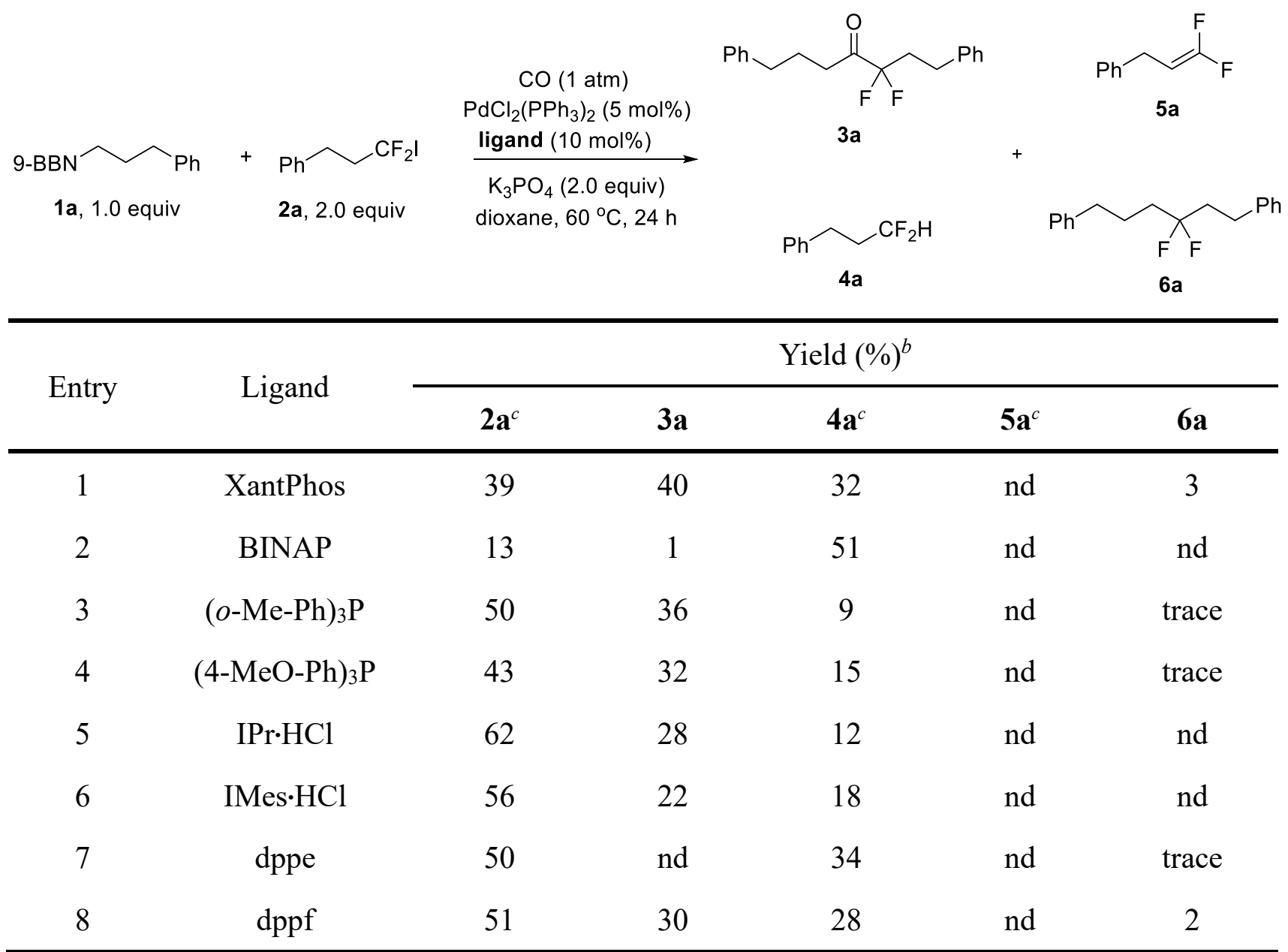

$\overline{{ }^{a} \text { Reaction conditions: 1a }\left(0.4 \mathrm{mmol}, 1.0 \text { equiv), } \mathbf{2 a}\left(0.8 \mathrm{mmol}, 2.0 \text { equiv), dioxane }(2.0 \mathrm{~mL}), 24 \mathrm{~h}, 60^{\circ} \mathrm{C} .{ }^{b} \text { Determined }\right.\right.}$ by ${ }^{19} \mathrm{~F}$ NMR using fluorobenzene as the internal standard; nd, not detected. ${ }^{c}$ The yields of $\mathbf{2 a}, \mathbf{4 a}, \mathbf{5 a}$ are calculated based on $\mathbf{2 a}$. 
Table S4. Screening of the bases. ${ }^{a}$

\begin{tabular}{|c|c|c|c|c|c|c|}
\hline \multirow{2}{*}{ 1a, 1.0 equiv } & \multirow{2}{*}{$\begin{aligned}+ & \mathrm{Ph} \sim \mathrm{CF}_{2} \mathrm{I} \\
& \mathbf{2 a}, 2.0 \text { equiv }\end{aligned}$} & \multirow{2}{*}{\multicolumn{2}{|c|}{$\begin{array}{c}\mathrm{CO}(1 \mathrm{~atm}) \\
\mathrm{PdCl}_{2}\left(\mathrm{PPh}_{3}\right)_{2}(5 \mathrm{~mol} \%) \\
\mathrm{XantPhos}(10 \mathrm{~mol} \%) \\
\begin{array}{c}\text { base }(2.0 \text { equiv }) \\
\text { dioxane, } 60^{\circ} \mathrm{C}, 24 \mathrm{~h}\end{array}\end{array}$}} & & \multirow{2}{*}{+} & a \\
\hline & & & & & & \\
\hline \multirow{2}{*}{ Entry } & \multirow{2}{*}{ Base } & \multicolumn{5}{|c|}{ Yield $(\%)^{b}$} \\
\hline & & $\mathbf{2} \mathbf{a}^{c}$ & $\mathbf{3 a}$ & $4 \mathbf{a}^{c}$ & $\mathbf{5} \mathbf{a}^{c}$ & 6 \\
\hline 1 & $\mathrm{Na}_{2} \mathrm{CO}_{3}$ & 65 & nd & 13 & nd & nc \\
\hline 2 & $\mathrm{~K}_{2} \mathrm{CO}_{3}$ & 62 & 4 & 12 & 1 & nc \\
\hline 3 & $\mathrm{Cs}_{2} \mathrm{CO}_{3}$ & 3 & 21 & 19 & 32 & nc \\
\hline 4 & $\mathrm{~K}_{3} \mathrm{PO}_{4}$ & 39 & 40 & 32 & nd & 3 \\
\hline 5 & $\mathrm{NaOMe}$ & 56 & 6 & 18 & 4 & 6 \\
\hline 6 & KOMe & 40 & 32 & 16 & 3 & 7 \\
\hline 7 & $\mathrm{KO} t \mathrm{Bu}$ & nd & nd & nd & 90 & nc \\
\hline $8^{c}$ & $\mathrm{~K}_{3} \mathrm{PO}_{4}$ & 31 & 56 & 25 & nd & 4 \\
\hline
\end{tabular}

${ }^{a}$ Reaction conditions: 1a $\left(0.4 \mathrm{mmol}, 1.0\right.$ equiv), $\mathbf{2 a}\left(0.8 \mathrm{mmol}, 2.0\right.$ equiv), dioxane $(2.0 \mathrm{~mL}), 24 \mathrm{~h}, 60^{\circ} \mathrm{C} .{ }^{b}$ Determined by ${ }^{19} \mathrm{~F}$ NMR using fluorobenzene as the internal standard; nd, not detected. ${ }^{C} \mathrm{~K}_{3} \mathrm{PO}_{4}(1.2 \mathrm{mmol}, 3.0$ equiv) was used. ${ }^{c}$ The yields of $\mathbf{2 a}, \mathbf{4 a}, \mathbf{5 a}$ were calculated based on $\mathbf{2 a}$. 
Table S5. Screening of the loading amount of $\mathrm{H}_{2} \mathrm{O}{ }^{a}$

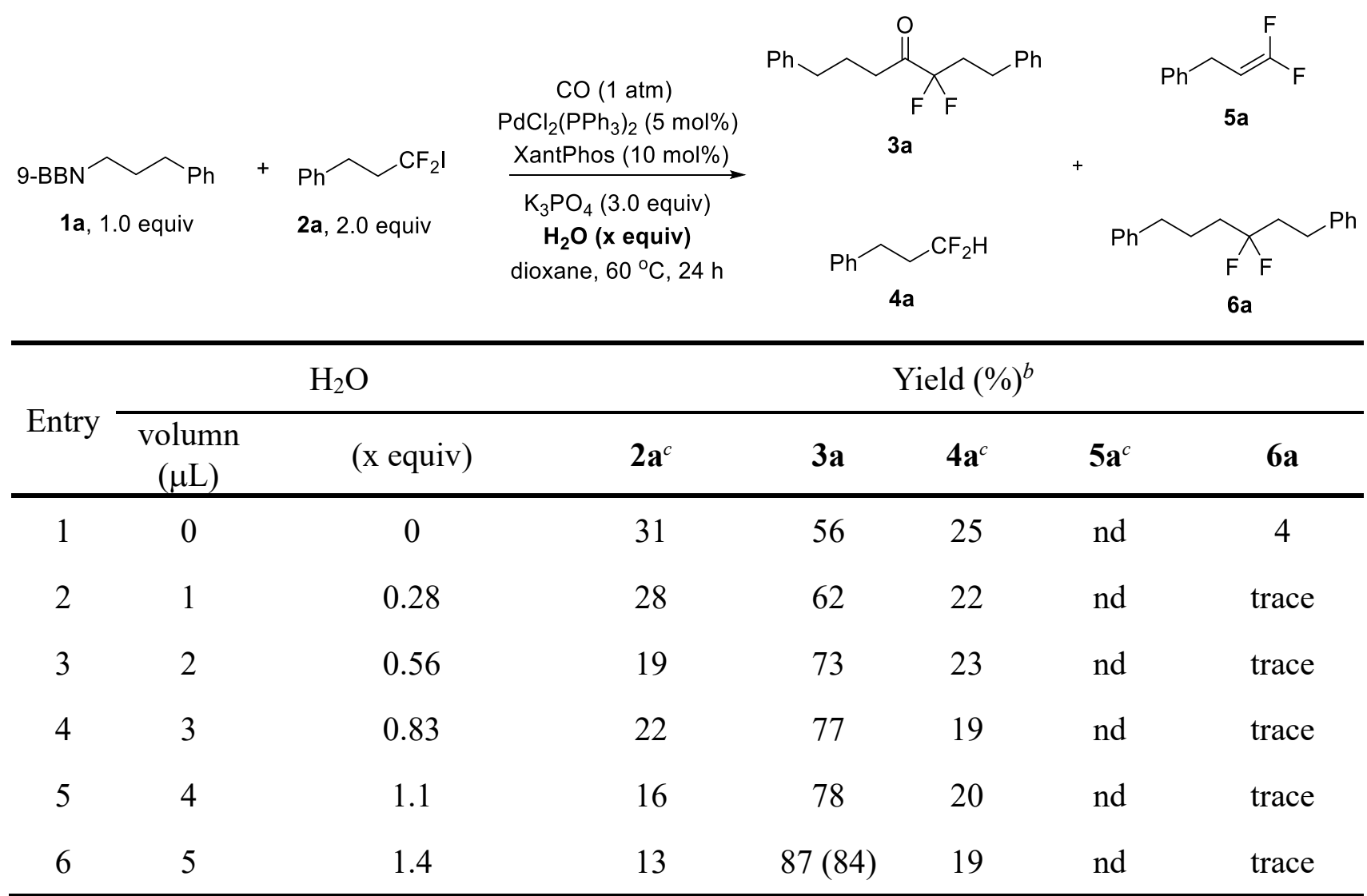

$\overline{{ }^{a} \text { Reaction conditions: 1a }\left(0.4 \mathrm{mmol}, 1.0 \text { equiv), } 2 \mathbf{a}\left(0.8 \mathrm{mmol}, 2.0 \text { equiv), dioxane }(2.0 \mathrm{~mL}), 24 \mathrm{~h}, 80^{\circ} \mathrm{C} .{ }^{b} \text { Determined }\right.\right.}$ by ${ }^{19} \mathrm{~F}$ NMR using fluorobenzene as internal standard and number in parenthesis is isolated yield; nd, not detected. ${ }^{c}$ The yields of $\mathbf{2 a}, \mathbf{4 a}, \mathbf{5 a}$ were calculated based on $\mathbf{2 a}$. 
Table S6. Control experiments ${ }^{a}$

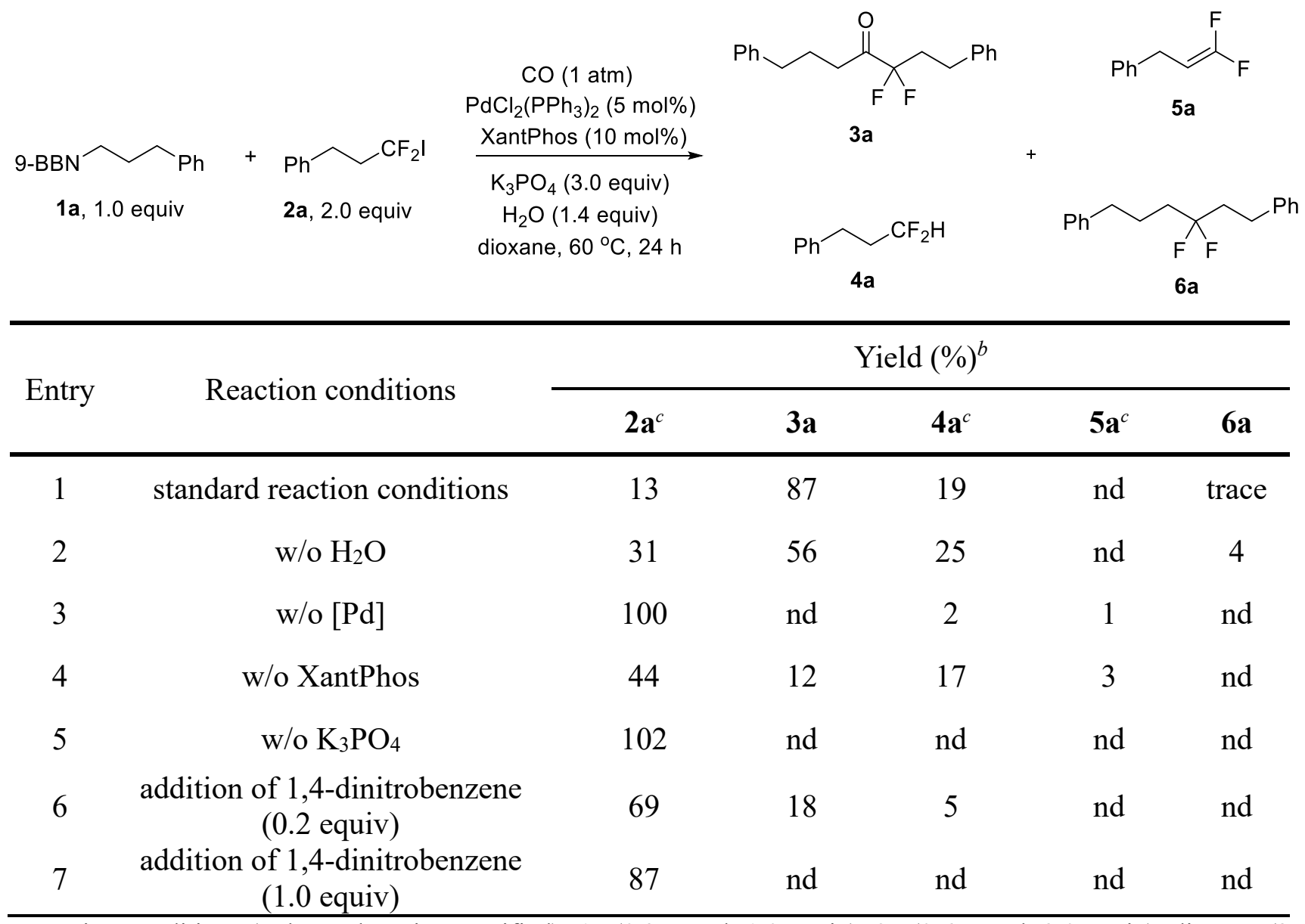

${ }^{a}$ Reaction conditions (unless otherwise specified): 1a $(0.3 \mathrm{mmol}, 1.0$ equiv), 2a (0.6 mmol, 2.0 equiv), dioxane (2 $\mathrm{mL}) .{ }^{b}$ Determined by ${ }^{19} \mathrm{~F}$ NMR using fluorobenzene as the internal standard; nd, not detected. ${ }^{c}$ The yields of 2a, 4a, 5a were calculated based on $\mathbf{2 a}$. 
Table S7. Ligand effect on the Pd-catalyzed carbonylation of $1 \mathrm{a}$ with $\mathbf{2 j}$. $^{a}$

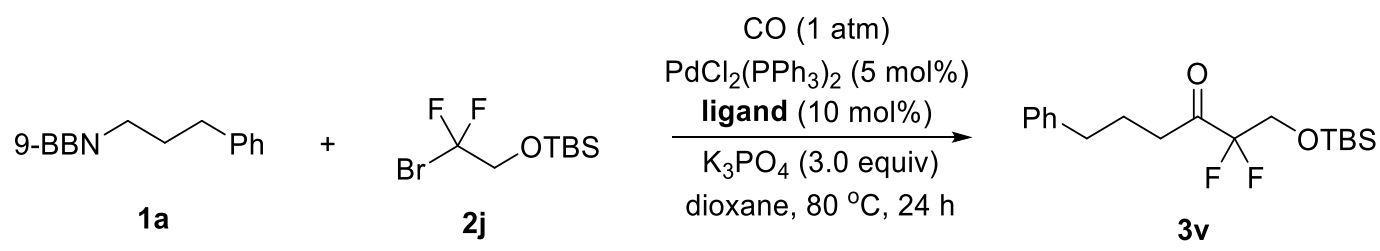

\begin{tabular}{ccc}
\hline Entry & Ligand & Yield $(\%)^{b}$ \\
\hline 1 & IPr·HCl & 15 \\
$2^{c}$ & IPr.HCl & 30 \\
3 & IMes·HCl & nd \\
4 & SIMes.HCl & nd \\
5 & SIPr·HCl & trace \\
6 & 1,3-Bis(1-adamantyl) imidazolinium chloride & nd \\
7 & XantPhos & trace \\
8 & ditBu-XantPhos & nd \\
9 & dppp & nd \\
10 & dppf & nd \\
\hline
\end{tabular}

${ }^{a}$ Reaction conditions: 1a ( $0.4 \mathrm{mmol}, 1.0$ equiv), 2 a $\left(0.8 \mathrm{mmol}, 2.0\right.$ equiv), dioxane $(2 \mathrm{~mL}), 24 \mathrm{~h} .{ }^{b}$ Determined by ${ }^{19} \mathrm{~F}$

NMR using fluorobenzene as the internal standard; nd, not detected. ${ }^{c}$ Reaction run at $100{ }^{\circ} \mathrm{C}$. 
Table S8. Screening palladium sources on the Pd-catalyzed carbonylation of 1 a with 2 l. $^{a}$

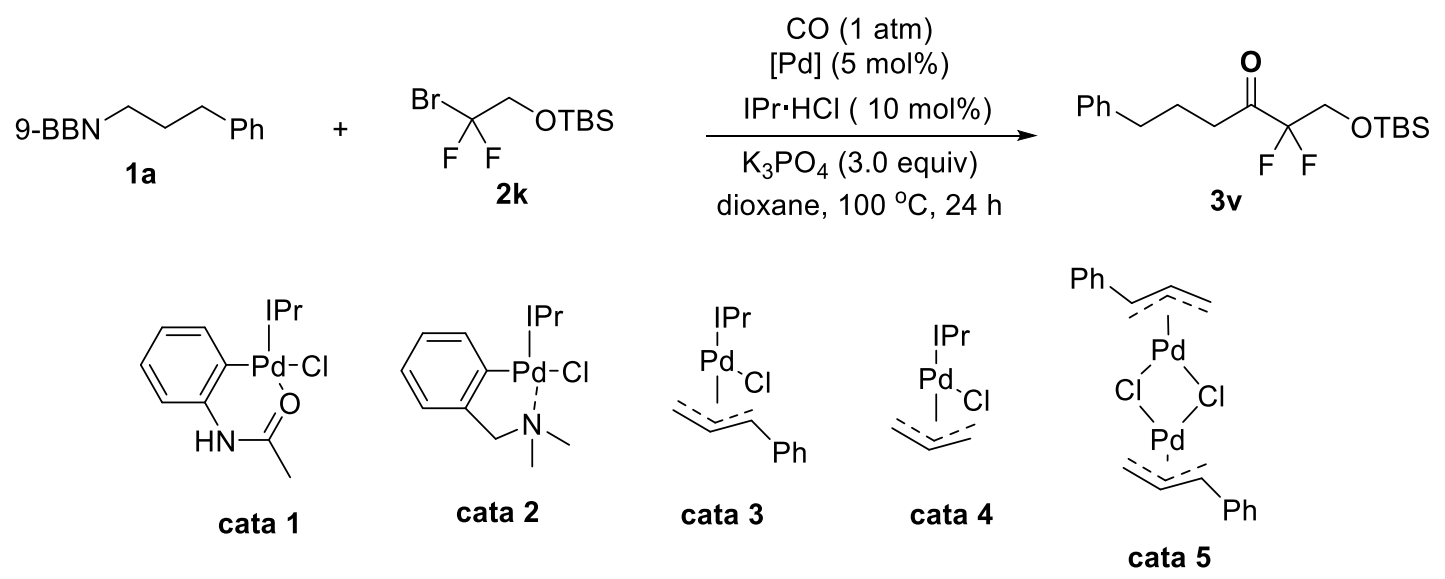

\begin{tabular}{ccc}
\hline Entry & {$[\mathrm{Pd}](5 \mathrm{~mol} \%)$} & Yield $(\%)^{b}$ \\
\hline 1 & $\mathrm{Pd}^{b}\left(\mathrm{PPh}_{3}\right)_{4}$ & 13 \\
2 & $\mathrm{PdCl}_{2}\left(\mathrm{PPh}_{3}\right)_{2}$ & 30 \\
3 & $\mathrm{Pd}_{2}(\mathrm{dba})_{3}$ & nd \\
4 & {$\left[\mathrm{PdCl}_{3}\left(\mathrm{C}_{3} \mathrm{H}_{5}\right)\right]_{2}$} & 44 \\
5 & cata 1 & 40 \\
6 & cata 2 & 12 \\
7 & cata 3 & 41 \\
8 & cata 4 & 42 \\
9 & cata 5 & 40 \\
\hline
\end{tabular}

${ }^{a}$ Reaction conditions: 1a (0.4 mmol, 1.0 equiv), 2a ( $0.8 \mathrm{mmol}, 2.0$ equiv), dioxane (2.0 mL), 24 h. ${ }^{b}$ Determined by ${ }^{19} \mathrm{~F}$ NMR using fluorobenzene as the internal standard; nd, not detected. 
Table S9. Optimizations of the Pd-Catalyzed Carbonylation of 1a with $2 \mathbf{k} .^{a}$

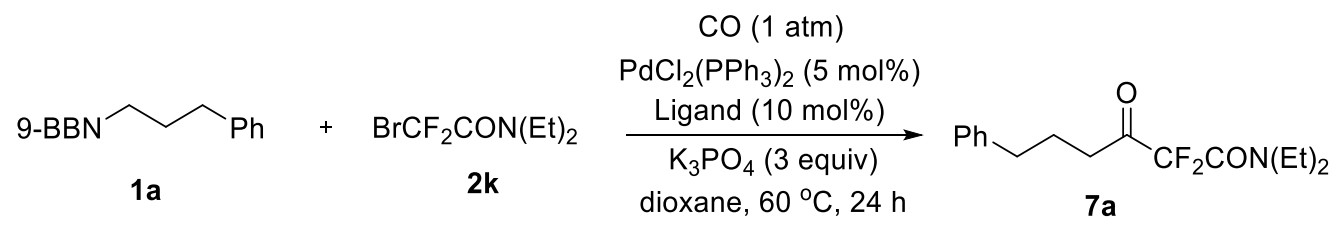

\begin{tabular}{ccccc}
\hline Entry & Ligand & $i$ PrOH/(equiv) & NaI/(equiv) & ${\text { Yield }(\%)^{b}}^{b}$ \\
\hline 1 & XantPhos & $/$ & $/$ & 13 \\
2 & IMes $\cdot \mathrm{HCl}$ & $/$ & $/$ & 64 \\
3 & dppf & $/$ & 0.25 & 4 \\
4 & $\mathrm{IMes} \cdot \mathrm{HCl}$ & 2.0 & 0.5 & 71 \\
5 & $\mathrm{IMes} \cdot \mathrm{HCl}$ & 2.0 & 0.75 & 77 \\
6 & $\mathrm{IMes} \cdot \mathrm{HCl}$ & 2.0 & 0.5 & 71 \\
7 & $\mathrm{IMes} \cdot \mathrm{HCl}$ & 1.5 & 0.5 & 73 \\
8 & $\mathrm{IMes} \cdot \mathrm{HCl}$ & 3.0 & $84^{c}$
\end{tabular}

${ }^{a}$ Reaction conditions: 1a ( $0.4 \mathrm{mmol}, 1.0$ equiv), $\mathbf{2 a}\left(0.8 \mathrm{mmol}, 2.0\right.$ equiv), dioxane $(2 \mathrm{~mL}), 24 \mathrm{~h} .{ }^{b}$ Determined by ${ }^{19} \mathrm{~F}$ NMR using fluorobenzene as internal standard. ${ }^{c}$ Isolated yield. 


\section{Radical clock experiment.}
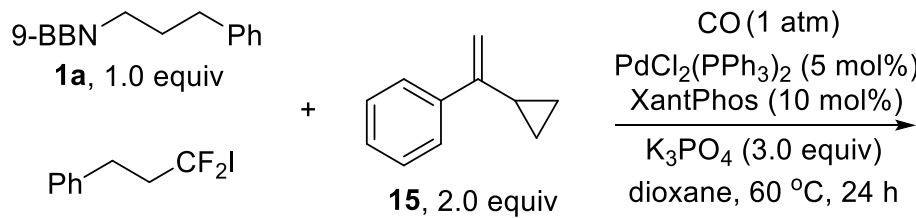

2a, 2.0 equiv

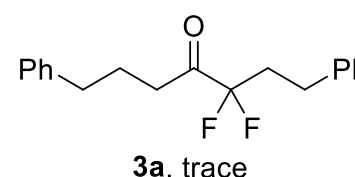

3a, trace

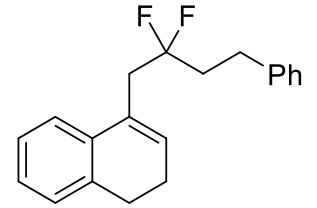

$16,84 \%$

Procedure: To a $25 \mathrm{~mL}$ of Schlenck tube were added $\mathrm{PdCl}_{2}\left(\mathrm{PPh}_{3}\right)_{2}(0.02 \mathrm{mmol}, 5 \mathrm{~mol} \%)$, XantPhos (0.04 mmol, $10 \mathrm{~mol} \%)$ under air, followed by $\mathrm{K}_{3} \mathrm{PO}_{4}$ (1.2 mmol, 3.0 equiv). The reaction mixture was then evacuated and backfilled with Ar (3 times). The mixture was evacuated again and backfilled with carbon monoxide CO (1 atm, balloon). 1a ( 0.4 mmol, 1.0 equiv), 2a ( 0.8 mmol, 2.0 equiv), 15 (0.8 mmol, 2.0 equiv) and dioxane $(2.0 \mathrm{~mL})$ were added subsequently. The Schlenck tube was screw capped and put into a preheated oil bath $\left(60^{\circ} \mathrm{C}\right)$. After stirring for $24 \mathrm{~h}$, the reaction mixture was cooled to room temperature. The resulting mixture was extracted with ethyl acetate, the combined organic layers were washed with brine, dried over $\mathrm{Na}_{2} \mathrm{SO}_{4}$, filtered and concentrated. The residue was purified with silica gel chromatography to give the product 16.

4-(2,2-Difluoro-4-phenylbutyl)-1,2-dihydronaphthalene (16). Compound $\mathbf{1 6}$ (201 mg, 84\% yield) as a colorless oil was purified with silica gel chromatography (Petroleum ether: Ethyl acetate $=100$ : 1). ${ }^{1} \mathrm{H}$ NMR (400 MHz, $\left.\mathrm{CDCl}_{3}\right) \delta 7.42(\mathrm{t}, J=6.8 \mathrm{~Hz}, 2 \mathrm{H}), 7.38-7.29(\mathrm{~m}, 3 \mathrm{H}), 7.27-7.21(\mathrm{~m}, 2 \mathrm{H})$, $7.11(\mathrm{~d}, J=7.2 \mathrm{~Hz}, 2 \mathrm{H}), 5.69(\mathrm{t}, J=7.2 \mathrm{~Hz}, 1 \mathrm{H}), 3.15(\mathrm{t}, J=6.8 \mathrm{~Hz}, 2 \mathrm{H}), 3.04(\mathrm{t}, J=15.2 \mathrm{~Hz}, 2 \mathrm{H})$, $2.77-2.70(\mathrm{~m}, 2 \mathrm{H}), 2.64(\mathrm{q}, J=7.2 \mathrm{~Hz}, 2 \mathrm{H}), 2.19-2.04(\mathrm{~m}, 2 \mathrm{H}) .{ }^{19} \mathrm{~F}$ NMR $\left(376 \mathrm{MHz}, \mathrm{CDCl}_{3}\right) \delta-$ $95.0(\mathrm{~m}, J=15.8 \mathrm{~Hz}, 2 \mathrm{~F}) .{ }^{13} \mathrm{C} \mathrm{NMR}\left(101 \mathrm{MHz}, \mathrm{CDCl}_{3}\right) \delta 140.5,139.8,135.4(\mathrm{t}, J=5.1 \mathrm{~Hz}), 132.1$, $128.44,128.39,128.29,128.26,128.2,127.2,126.6,126.1,123.5$ (t, $J=244.4 \mathrm{~Hz}), 45.6$ (t, $J=26.3$ $\mathrm{Hz}), 38.1(\mathrm{t}, J=25.3 \mathrm{~Hz}), 32.6,28.4(\mathrm{t}, J=5.1 \mathrm{~Hz}), 5.2 . \mathrm{MS}(\mathrm{FI}): \mathrm{m} / \mathrm{z}(\%) 298(\mathrm{M})^{+} . \mathrm{HRMS}(\mathrm{FI}) \mathrm{m} / \mathrm{z}$ : $[\mathrm{M}]^{+}$Calcd for $\mathrm{C}_{20} \mathrm{H}_{20} \mathrm{~F}_{2}: 298.1528$; Found: 298.1531 .

\section{General procedure for the Pd-catalyzed carbonylation of alkyl-9-BBN 1 with difluoroalkyl halides 2 under $1 \mathrm{~atm}$ CO. \\ General Procedure A: Pd-catalyzed carbonylation of alkyl-9-BBN 1 with unactivated difluoroalkyl iodides $2 \mathrm{a}-\mathrm{i}$.}

To a $25 \mathrm{~mL}$ of Schlenck tube were added $\mathrm{PdCl}_{2}\left(\mathrm{PPh}_{3}\right)_{2}(0.02 \mathrm{mmol}, 5 \mathrm{~mol} \%)$, XantPhos (0.04 mmol, $10 \mathrm{~mol} \%$ ) under air, followed by $\mathrm{K}_{3} \mathrm{PO}_{4}(1.2 \mathrm{mmol}, 3.0$ equiv). The reaction mixture was then evacuated and backfilled with Ar (3 times). The mixture was evacuated again and backfilled with carbon monoxide CO (1 atm, balloon). Alkyl-9-BBN 1 (0.4 mmol, 1.0 equiv), compound 2 (0.8 mmol, 
2.0 equiv) and dioxane $(2.0 \mathrm{~mL})$ were added subsequently. The Schlenck tube was screw capped and put into a preheated oil bath $\left(60^{\circ} \mathrm{C}\right)$. After stirring for $24 \mathrm{~h}$, the reaction mixture was cooled to room temperature. The resulting mixture was extracted with ethyl acetate, the combined organic layers were washed with brine, dried over $\mathrm{Na}_{2} \mathrm{SO}_{4}$, filtered and concentrated. The residue was purified with silica gel chromatography to give product 3,9 or 11 .

Note: The resulting difluoroketones are stable during working up and isolation. CO is toxic, for safety, the reaction should be conducted with a ventilation system.

\section{General Procedure B: Pd-catalyzed carbonylation of alkyl-9-BBN 1 with difluoroalkyl bromides} 2k-p.

To a $25 \mathrm{~mL}$ of Schlenck tube were added $\mathrm{PdCl}_{2}\left(\mathrm{PPh}_{3}\right)_{2}(0.02 \mathrm{mmol}, 5 \mathrm{~mol} \%)$, IMes $\cdot \mathrm{HCl}(0.04 \mathrm{mmol}$, $10 \mathrm{~mol} \%$ ) under air, followed by $\mathrm{K}_{3} \mathrm{PO}_{4}$ ( $1.2 \mathrm{mmol}, 3.0$ equiv), $\mathrm{NaI}(0.2 \mathrm{mmol}, 0.5$ equiv). The reaction mixture was then evacuated and backfilled with Ar (3 times). The mixture was evacuated again and backfilled with carbon monoxide CO (1 atm, balloon). Alkyl-9-BBN 1 (0.4 mmol, 1.0 equiv), compound 2 ( $0.8 \mathrm{mmol}, 2.0$ equiv), $i \mathrm{PrOH}$ (1.2 mmol, 3.0 equiv) and dioxane $(2 \mathrm{~mL})$ were added subsequently. The Schlenck tube was screw capped and put into a preheated oil bath $\left(60^{\circ} \mathrm{C}\right)$. After stirring for $24 \mathrm{~h}$, the reaction mixture was cooled to room temperature. The resulting mixture was extracted with ethyl acetate, the combined organic layers were washed with brine, dried over $\mathrm{Na}_{2} \mathrm{SO}_{4}$, filtered and concentrated. The residue was purified with silica gel chromatography to give product 7 .

Note: The resulting difluoroketones are stable during working up and isolation.

\section{Characterization data for compounds 3 .}<smiles>O=C(CCCc1ccccc1)C(F)(F)CCc1ccccc1</smiles>

3,3-Difluoro-1,7-diphenylheptan-4-one (3a). The reaction was carried out using General Procedure A. Compound 3a (102 mg, 84\% yield) as a colorless oil was purified with silica gel chromatography $\left(\right.$ Petroleum ether: Ethyl acetate = 30: 1). ${ }^{1} \mathrm{H}$ NMR $\left(400 \mathrm{MHz}, \mathrm{CDCl}_{3}\right) \delta 7.37-7.32(\mathrm{~m}, 4 \mathrm{H}), 7.29-$ $7.21(\mathrm{~m}, 6 \mathrm{H}), 2.85-2.79(\mathrm{~m}, 2 \mathrm{H}), 2.76-2.66(\mathrm{~m}, 4 \mathrm{H}), 2.41-2.26(\mathrm{~m}, 2 \mathrm{H}), 2.05-1.96(\mathrm{~m}, 2 \mathrm{H}) .{ }^{19} \mathrm{~F}$ $\operatorname{NMR}\left(376 \mathrm{MHz}, \mathrm{CDCl}_{3}\right) \delta-107.5(\mathrm{t}, J=17.3 \mathrm{~Hz}, 2 \mathrm{~F}) .{ }^{13} \mathrm{C} \mathrm{NMR}\left(126 \mathrm{MHz}, \mathrm{CDCl}_{3}\right) \delta 200.8(\mathrm{t}, J=$ 31.4 Hz), 141.1, 139.8, 128.6, 128.42, 128.40, 128.2, 126.4, 126.1, 117.7 (t, $J=252.8 \mathrm{~Hz}), 35.4,34.8$, $34.3(\mathrm{t}, J=22.9 \mathrm{~Hz}), 27.5(\mathrm{t}, J=4.9 \mathrm{~Hz}), 24.1$. MS (DART): $\mathrm{m} / \mathrm{z}(\%) 320\left(\mathrm{M}+\mathrm{NH}_{4}\right)^{+}$. HRMS (DART) $\mathrm{m} / \mathrm{z}:\left[\mathrm{M}+\mathrm{NH}_{4}\right]^{+}$Calcd for $\mathrm{C}_{19} \mathrm{H}_{24} \mathrm{NOF}_{2}: 320.1820$; Found: 320.1815 . 


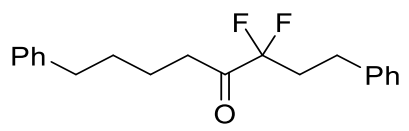

3,3-Difluoro-1,8-diphenyloctan-4-one (3b). The reaction was carried out using General Procedure A. Compound 3b (99 mg, 78\% yield) as a colorless oil was purified with silica gel chromatography $($ Petroleum ether: Ethyl acetate $=80: 1) .{ }^{1} \mathrm{H}$ NMR $\left(400 \mathrm{MHz}, \mathrm{CDCl}_{3}\right) \delta 7.33-7.26(\mathrm{~m}, 4 \mathrm{H}), 7.24-$ $7.16(\mathrm{~m}, 6 \mathrm{H}), 2.81-2.75(\mathrm{~m}, 2 \mathrm{H}), 2.70(\mathrm{t}, J=5.6 \mathrm{~Hz}, 2 \mathrm{H}), 2.64(\mathrm{t}, J=6.8 \mathrm{~Hz}, 2 \mathrm{H}), 2.36-2.22(\mathrm{~m}$, 2H), $1.72-1.60(\mathrm{~m}, 4 \mathrm{H}) .{ }^{19} \mathrm{~F}$ NMR $\left(376 \mathrm{MHz}, \mathrm{CDCl}_{3}\right) \delta-107.5(\mathrm{t}, J=17.3 \mathrm{~Hz}, 2 \mathrm{~F}) .{ }^{13} \mathrm{C}$ NMR $(101$ $\left.\mathrm{MHz}, \mathrm{CDCl}_{3}\right) \delta 200.9(\mathrm{t}, J=31.5 \mathrm{~Hz}), 141.9,139.8,128.6,128.34,128.32,128.26,126.4,125.8,117.7$ $(\mathrm{t}, J=253.4 \mathrm{~Hz}), 36.1,35.6,34.4(\mathrm{t}, J=22.9 \mathrm{~Hz}), 30.7,27.5(\mathrm{t}, J=4.9 \mathrm{~Hz}), 22.2 . \mathrm{MS}(\mathrm{DART}): \mathrm{m} / \mathrm{z}$ (\%) $334\left(\mathrm{M}+\mathrm{NH}_{4}\right)^{+}$. HRMS (DART) m/z: $\left[\mathrm{M}+\mathrm{NH}_{4}\right]^{+}$Calcd for $\mathrm{C}_{20} \mathrm{H}_{26} \mathrm{NOF}_{2}$ : 334.1977; Found: 334.1976.

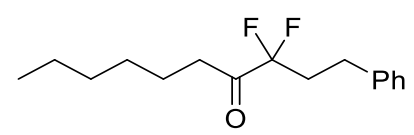

3,3-Difluoro-1-phenyldecan-4-one. (3c). The reaction was carried out using General Procedure A. Compound 3c (75 mg, 70\% yield) as a colorless oil was purified with silica gel chromatography (Petroleum ether: Ethyl acetate $=40: 1) .{ }^{1} \mathrm{H}$ NMR $\left(400 \mathrm{MHz}, \mathrm{CDCl}_{3}\right) \delta 7.31(\mathrm{t}, J=7.6 \mathrm{~Hz}, 2 \mathrm{H}), 7.26$ $-7.19(\mathrm{~m}, 3 \mathrm{H}), 2.83-2.77(\mathrm{~m}, 2 \mathrm{H}), 2.68(\mathrm{t}, J=7.2 \mathrm{~Hz}, 2 \mathrm{H}), 2.39-2.24(\mathrm{~m}, 2 \mathrm{H}), 1.67-1.58(\mathrm{~m}, 2 \mathrm{H})$, $1.38-1.26(\mathrm{~m}, 6 \mathrm{H}), 0.91(\mathrm{t}, J=6.8 \mathrm{~Hz}, 3 \mathrm{H}) .{ }^{19} \mathrm{~F}$ NMR $\left(376 \mathrm{MHz}, \mathrm{CDCl}_{3}\right) \delta-107.6(\mathrm{t}, J=17.3 \mathrm{~Hz}$, 2F). ${ }^{13} \mathrm{C}$ NMR $\left(126 \mathrm{MHz}, \mathrm{CDCl}_{3}\right) \delta 201.2(\mathrm{t}, J=31.4 \mathrm{~Hz}), 139.9,128.6,128.3,126.4,117.7(\mathrm{t}, J=$ $252.9 \mathrm{~Hz}), 36.3,34.4(\mathrm{t}, J=22.9 \mathrm{~Hz}), 31.5,28.6,27.6$ (t, $J=4.8 \mathrm{~Hz}), 22.6,22.4,14.0$. MS (DART): $\mathrm{m} / \mathrm{z}(\%) 286\left(\mathrm{M}+\mathrm{NH}_{4}\right)^{+}$. HRMS (DART) m/z: $\left[\mathrm{M}+\mathrm{NH}_{4}\right]^{+}$Calcd for $\mathrm{C}_{16} \mathrm{H}_{26} \mathrm{NOF}_{2}: 286.1977$; Found: 286.1980 .

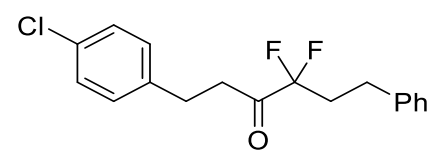

1-(4-Chlorophenyl)-4,4-difluoro-6-phenylhexan-3-one (3d). The reaction was carried out using General Procedure A. Compound 3d (97 mg, 75\% yield) as a yellow oil was purified with silica gel chromatography (Petroleum ether: Ethyl acetate $=50: 1) .{ }^{1} \mathrm{H}$ NMR $\left(400 \mathrm{MHz}, \mathrm{CDCl}_{3}\right) \delta 7.33-7.21$ $(\mathrm{m}, 5 \mathrm{H}), 7.18-7.10(\mathrm{~m}, 4 \mathrm{H}), 2.98(\mathrm{t}, J=7.2 \mathrm{~Hz}, 2 \mathrm{H}), 2.89(\mathrm{t}, J=7.2 \mathrm{~Hz}, 2 \mathrm{H}), 2.77-2.71(\mathrm{~m}, 2 \mathrm{H})$, $2.35-2.20(\mathrm{~m}, 2 \mathrm{H}) .{ }^{19} \mathrm{~F}$ NMR $\left(376 \mathrm{MHz}, \mathrm{CDCl}_{3}\right) \delta-107.4(\mathrm{t}, J=17.3 \mathrm{~Hz}, 2 \mathrm{~F}) .{ }^{13} \mathrm{C} \mathrm{NMR}(101 \mathrm{MHz}$, $\left.\mathrm{CDCl}_{3}\right) \delta 199.8(\mathrm{t}, J=31.9 \mathrm{~Hz}), 139.7,138.6,132.2,129.7,128.63,128.58,128.2,126.4,117.7(\mathrm{t}, J$ 
$=253.5 \mathrm{~Hz}), 37.7,34.3(\mathrm{t}, J=22.9 \mathrm{~Hz}), 27.9,27.5(\mathrm{t}, J=4.8 \mathrm{~Hz}) . \mathrm{MS}(\mathrm{DART}): \mathrm{m} / \mathrm{z}(\%) 340\left(\mathrm{M}+\mathrm{NH}_{4}\right)^{+}$. HRMS (DART) m/z: [M+NH$]^{+}$Calcd for $\mathrm{C}_{18} \mathrm{H}_{21} \mathrm{NOF}_{2} \mathrm{Cl}$ : 340.1274; Found: 340.1276.

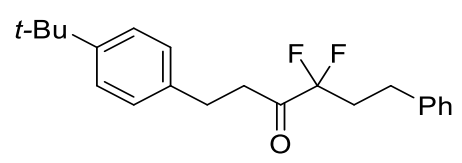

1-(4-(tert-Butyl)phenyl)-4,4-difluoro-6-phenylhexan-3-one (3e). The reaction was carried out using General Procedure A. Compound $\mathbf{3 e}(80 \mathrm{mg}, 58 \%$ yield) as a colorless oil was purified with silica gel chromatography (Petroleum ether: Ethyl acetate $=80: 1) .{ }^{1} \mathrm{H}$ NMR $\left(400 \mathrm{MHz}, \mathrm{CDCl}_{3}\right) \delta 7.34-$ $7.27(\mathrm{~m}, 4 \mathrm{H}), 7.24-7.11(\mathrm{~m}, 5 \mathrm{H}), 3.00(\mathrm{t}, J=7.6 \mathrm{~Hz}, 2 \mathrm{H}), 2.90(\mathrm{t}, J=7.6 \mathrm{~Hz}, 2 \mathrm{H}), 2.77-2.71(\mathrm{~m}$, 2H), $2.35-2.21(\mathrm{~m}, 2 \mathrm{H}), 1.30(\mathrm{~s}, 9 \mathrm{H}) .{ }^{19} \mathrm{~F} \mathrm{NMR}\left(376 \mathrm{MHz}, \mathrm{CDCl}_{3}\right) \delta-107.5(\mathrm{t}, J=16.9 \mathrm{~Hz}, 2 \mathrm{~F}) .{ }^{13} \mathrm{C}$ NMR (101 MHz, $\left.\mathrm{CDCl}_{3}\right) \delta 200.3(\mathrm{t}, J=31.4 \mathrm{~Hz}), 149.2,139.8,137.0,128.6,128.3,128.0,126.4$, 125.5, $117.7(\mathrm{t}, J=253.6 \mathrm{~Hz}), 38.1,34.42(\mathrm{t}, J=23.2 \mathrm{~Hz}), 34.36,31.3,28.0,27.5(\mathrm{t}, J=4.7 \mathrm{~Hz}) . \mathrm{MS}$ (DART): m/z (\%) $362\left(\mathrm{M}+\mathrm{NH}_{4}\right)^{+}$. HRMS (DART) m/z: [M+NH$]^{+}$Calcd for $\mathrm{C}_{22} \mathrm{H}_{30} \mathrm{NOF}_{2}: 362.2290$; Found: 362.2288 .<smiles>CC(F)(F)OCCCCC(=O)C(F)(F)CCc1ccccc1</smiles>

8-((tert-Butyldimethylsilyl)oxy)-3,3-difluoro-1-phenylheptan-4-one (3f). The reaction was carried out using General Procedure A. Compound 3f (126 mg, 85\% yield) as a colorless oil was purified with silica gel chromatography (Petroleum ether: Ethyl acetate $=40: 1) .{ }^{1} \mathrm{H}$ NMR $\left(400 \mathrm{MHz}, \mathrm{CDCl}_{3}\right)$ $\delta 7.31(\mathrm{t}, J=7.2 \mathrm{~Hz}, 2 \mathrm{H}), 7.25-7.19(\mathrm{~m}, 3 \mathrm{H}), 3.64(\mathrm{t}, J=6.0 \mathrm{~Hz}, 2 \mathrm{H}), 2.82-2.77(\mathrm{~m}, 2 \mathrm{H}), 2.73(\mathrm{t}, J$ $=7.2 \mathrm{~Hz}, 2 \mathrm{H}), 2.38-2.24(\mathrm{~m}, 2 \mathrm{H}), 1.74-1.65(\mathrm{~m}, 2 \mathrm{H}), 1.59-1.51(\mathrm{~m}, 2 \mathrm{H}), 0.91(\mathrm{~s}, 9 \mathrm{H}), 0.07(\mathrm{~s}$, $6 \mathrm{H}) .{ }^{19} \mathrm{~F}$ NMR $\left(376 \mathrm{MHz}, \mathrm{CDCl}_{3}\right) \delta-107.6(\mathrm{t}, J=17.3 \mathrm{~Hz}, 2 \mathrm{~F}) .{ }^{13} \mathrm{C} \mathrm{NMR}\left(101 \mathrm{MHz}, \mathrm{CDCl}_{3}\right) \delta 201.0$ $(\mathrm{t}, J=31.3 \mathrm{~Hz}), 139.9,128.6,128.3,126.4,117.7(\mathrm{t}, J=253.5 \mathrm{~Hz}), 62.7,36.0,34.4(\mathrm{t}, J=23.0 \mathrm{~Hz})$, 31.9, $27.6(\mathrm{t}, J=4.7 \mathrm{~Hz}), 25.9,19.2,18.3,-5.4 . \mathrm{MS}(\mathrm{FI}): \mathrm{m} / \mathrm{z}(\%) 370(\mathrm{M})^{+}, 342,313,215,199,181$, 133, 91 (100). HRMS (FI) m/z: [M] ${ }^{+}$Calcd for $\mathrm{C}_{20} \mathrm{H}_{32} \mathrm{O}_{2} \mathrm{~F}_{2} \mathrm{Si}$ : 370.2134; Found: 370.2131.

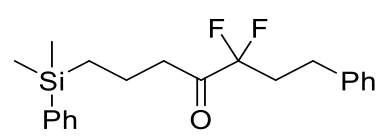

7-(Dimethyl(phenyl)silyl)-3,3-difluoro-1-phenylheptan-4-one. (3g). The reaction was carried out using General Procedure A. Compound 3g (91 mg, 63\% yield) as a colorless oil was purified with silica gel chromatography (Petroleum ether: Ethyl acetate $=40: 1) .{ }^{1} \mathrm{H}$ NMR $\left(400 \mathrm{MHz}, \mathrm{CDCl}_{3}\right) \delta 7.53$ 
- 7.49 (m, 2H), 7.38 - 7.34 (m, 3H), 7.33 - 7.27 (m, 2H), 7.24 - 7.17 (m, 3H), 2.80 - $2.74(\mathrm{~m}, 2 \mathrm{H})$, $2.68(\mathrm{t}, J=7.2 \mathrm{~Hz}, 2 \mathrm{H}), 2.35-2.20(\mathrm{~m}, 2 \mathrm{H}), 1.70-1.60(\mathrm{~m}, 2 \mathrm{H}), 0.79-0.73(\mathrm{~m}, 2 \mathrm{H}), 0.30(\mathrm{~s}, 6 \mathrm{H})$. ${ }^{19} \mathrm{~F} \mathrm{NMR}\left(376 \mathrm{MHz}, \mathrm{CDCl}_{3}\right) \delta-107.7(\mathrm{t}, J=17.3 \mathrm{~Hz}, 2 \mathrm{~F}) .{ }^{13} \mathrm{C} \mathrm{NMR}\left(126 \mathrm{MHz}, \mathrm{CDCl}_{3}\right) \delta 201.0(\mathrm{t}, J$ $=31.1 \mathrm{~Hz}), 139.9,138.8,133.5,129.0,128.6,128.3,127.8,126.4,117.7$ (t, $J=253.1 \mathrm{~Hz}), 39.8,34.4$ (t, $J=23.1 \mathrm{~Hz}), 27.5(\mathrm{t}, J=4.9 \mathrm{~Hz}), 17.4,15.5,-3.2 . \mathrm{MS}(\mathrm{DART}): \mathrm{m} / \mathrm{z}(\%) 378\left(\mathrm{M}+\mathrm{NH}_{4}\right)^{+}$. HRMS (DART) m/z: $\left[\mathrm{M}+\mathrm{NH}_{4}\right]^{+}$Calcd for $\mathrm{C}_{21} \mathrm{H}_{30} \mathrm{NOF}_{2} \mathrm{Si}$ : 378.2059; Found: 378.2058 .<smiles>CC(C)(C)OC(=O)CCCC(=O)C(F)(F)CCc1ccccc1</smiles>

tert-Butyl 6,6-difluoro-5-oxo-8-phenyloctanoate (3h). The reaction was carried out using General Procedure A. Compound $3 \mathrm{~h}$ (71 mg, 54\% yield) as a colorless oil was purified with silica gel chromatography (Petroleum ether: Ethyl acetate = 15: 1). ${ }^{1} \mathrm{H} \mathrm{NMR}\left(400 \mathrm{MHz}, \mathrm{CDCl}_{3}\right) \delta 7.30(\mathrm{t}, J=$ $7.2 \mathrm{~Hz}, 2 \mathrm{H}), 7.24-7.17(\mathrm{~m}, 3 \mathrm{H}), 2.81-2.72(\mathrm{~m}, 4 \mathrm{H}), 2.37-2.23(\mathrm{~m}, 4 \mathrm{H}), 1.94-1.85$ (m, 2H), 1.45 (s, 9H). ${ }^{19} \mathrm{~F} \mathrm{NMR}\left(376 \mathrm{MHz}, \mathrm{CDCl}_{3}\right) \delta-107.6(\mathrm{t}, J=16.9 \mathrm{~Hz}, 2 \mathrm{~F}) .{ }^{13} \mathrm{C} \mathrm{NMR}\left(126 \mathrm{MHz}, \mathrm{CDCl}_{3}\right) \delta$ $200.5(\mathrm{t}, J=31.6 \mathrm{~Hz}), 172.1,139.8,128.6,128.3,126.4,117.7(\mathrm{t}, J=252.8 \mathrm{~Hz}), 80.5,35.2,34.3(\mathrm{t}, J$ $=22.8 \mathrm{~Hz}), 34.1,28.1,27.5(\mathrm{t}, J=4.9 \mathrm{~Hz}), 18.1 . \mathrm{MS}(\mathrm{DART}): \mathrm{m} / \mathrm{z}(\%) 344\left(\mathrm{M}+\mathrm{NH}_{4}\right)^{+}$. HRMS (DART) $\mathrm{m} / \mathrm{z}:\left[\mathrm{M}+\mathrm{NH}_{4}\right]^{+}$Calcd for $\mathrm{C}_{18} \mathrm{H}_{28} \mathrm{NO}_{3} \mathrm{~F}_{2}$ : 344.2032; Found: 344.2031 .

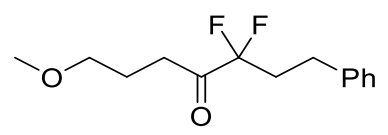

3,3-Difluoro-7-methoxy-1-phenylheptan-4-one. (3i). The reaction was carried out using General Procedure A. Compound 3i (60 mg, 58\% yield) as a colorless oil was purified with silica gel chromatography (Petroleum ether: Ethyl acetate = 30: 1). ${ }^{1} \mathrm{H}$ NMR $\left(400 \mathrm{MHz}, \mathrm{CDCl}_{3}\right) \delta 7.30(\mathrm{t}, J=$ $7.2 \mathrm{~Hz}, 2 \mathrm{H}), 7.24-7.18(\mathrm{~m}, 3 \mathrm{H}), 3.40(\mathrm{t}, J=6.0 \mathrm{~Hz}, 2 \mathrm{H}), 3.32(\mathrm{~s}, 3 \mathrm{H}), 2.82-2.76(\mathrm{~m}, 4 \mathrm{H}), 2.38-$ $2.23(\mathrm{~m}, 2 \mathrm{H}), 1.94-1.85(\mathrm{~m}, 2 \mathrm{H}) .{ }^{19} \mathrm{~F}$ NMR $\left(376 \mathrm{MHz}, \mathrm{CDCl}_{3}\right) \delta-107.7(\mathrm{t}, J=16.9 \mathrm{~Hz}, 2 \mathrm{~F}) .{ }^{13} \mathrm{C}$ NMR (126 MHz, $\left.\mathrm{CDCl}_{3}\right) \delta 200.9(\mathrm{t}, J=31.2 \mathrm{~Hz}), 139.9,128.6,128.3,126.4,117.7(\mathrm{t}, J=252.9 \mathrm{~Hz})$, 71.2, 58.5, $34.5(\mathrm{t}, J=22.9 \mathrm{~Hz}), 33.1,27.5(\mathrm{t}, J=4.9 \mathrm{~Hz}), 22.8$. MS (EI): m/z (\%) $256(\mathrm{M})^{+}, 236,218$, 152, 101 (100), 91, 69. HRMS (EI) m/z: [M] ${ }^{+}$Calcd for $\mathrm{C}_{14} \mathrm{H}_{18} \mathrm{O}_{2} \mathrm{~F}_{2}$ : 256.1269; Found: 256.1272.

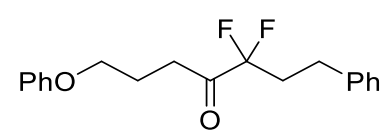

3,3-Difluoro-7-phenoxy-1-phenylheptan-4-one (3j). The reaction was carried out using General Procedure A. Compound 3j (76 mg, 60\% yield) as a yellow oil was purified with silica gel 
chromatography (Petroleum ether: Ethyl acetate = 30: 1). ${ }^{1} \mathrm{H}$ NMR $\left(400 \mathrm{MHz}, \mathrm{CDCl}_{3}\right) \delta 7.33-7.27$ $(\mathrm{m}, 4 \mathrm{H}), 7.25-7.17(\mathrm{~m}, 3 \mathrm{H}), 6.96(\mathrm{t}, J=7.2 \mathrm{~Hz}, 1 \mathrm{H}), 6.90(\mathrm{~d}, J=8.0 \mathrm{~Hz}, 2 \mathrm{H}), 4.01(\mathrm{t}, J=6.0 \mathrm{~Hz}$, 2H), $2.93(\mathrm{t}, J=6.8 \mathrm{~Hz}, 2 \mathrm{H}), 2.84-2.77(\mathrm{~m}, 2 \mathrm{H}), 2.40-2.26(\mathrm{~m}, 2 \mathrm{H}), 2.16-2.08(\mathrm{~m}, 2 \mathrm{H}) .{ }^{19} \mathrm{~F}$ NMR $\left(376 \mathrm{MHz}, \mathrm{CDCl}_{3}\right) \delta-107.5(\mathrm{t}, J=17.3 \mathrm{~Hz}, 2 \mathrm{~F}) .{ }^{13} \mathrm{C} \mathrm{NMR}\left(126 \mathrm{MHz}, \mathrm{CDCl}_{3}\right) \delta 200.7(\mathrm{t}, J=31.6 \mathrm{~Hz})$, 158.6, 139.8, 129.5, 128.6, 128.3, 126.4, 120.8, 117.7 (t, $J=252.8 \mathrm{~Hz}), 114.4$, 66.1, $34.4(\mathrm{t}, J=22.9$ Hz), 32.9, 27.5 (t, $J=4.7 \mathrm{~Hz})$, 22.4. MS (EI): m/z (\%) $318(\mathrm{M})^{+}, 225$, 205, 141, 91 (100), 77. HRMS (EI) $\mathrm{m} / \mathrm{z}:[\mathrm{M}]^{+}$Calcd for $\mathrm{C}_{19} \mathrm{H}_{20} \mathrm{O}_{2} \mathrm{~F}_{2}: 318.1426$; Found: 318.1425 .

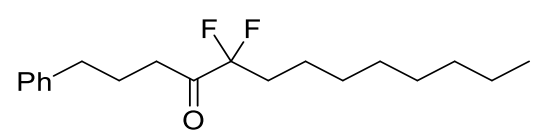

5,5-Difluoro-1-phenyltridecan-4-one (3k). The reaction was carried out using General Procedure A. Compound 3k (80 mg, 64\% yield) as a colorless oil was purified with silica gel chromatography $($ Petroleum ether: Ethyl acetate $=40: 1) .{ }^{1} \mathrm{H}$ NMR $\left(400 \mathrm{MHz}, \mathrm{CDCl}_{3}\right) \delta 7.30(\mathrm{t}, J=7.2 \mathrm{~Hz}, 2 \mathrm{H}), 7.24$ $-7.16(\mathrm{~m}, 3 \mathrm{H}), 2.72-2.63(\mathrm{~m}, 4 \mathrm{H}), 2.02-1.92(\mathrm{~m}, 4 \mathrm{H}), 1.47-1.37(\mathrm{~m}, 2 \mathrm{H}), 1.36-1.22(\mathrm{~m}, 10 \mathrm{H})$, $0.90(\mathrm{t}, J=6.4 \mathrm{~Hz}, 3 \mathrm{H}) .{ }^{19} \mathrm{~F}$ NMR $\left(376 \mathrm{MHz}, \mathrm{CDCl}_{3}\right) \delta-107.2(\mathrm{t}, J=17.7 \mathrm{~Hz}, 2 \mathrm{~F}) .{ }^{13} \mathrm{C}$ NMR $(101$ $\left.\mathrm{MHz} \mathrm{CDCl}_{3}\right) \delta 201.3(\mathrm{t}, J=31.6 \mathrm{~Hz}), 141.2,128.4,126.1,118.4(\mathrm{t}, J=252.6 \mathrm{~Hz}), 35.6,34.8,32.6(\mathrm{t}$, $J=23.0 \mathrm{~Hz}), 31.8,29.3,29.2,29.0,24.2,22.6,21.2(\mathrm{t}, J=4.2 \mathrm{~Hz}), 14.1 . \mathrm{MS}(\mathrm{EI}): \mathrm{m} / \mathrm{z}(\%) 310(\mathrm{M})^{+}$, 147 (100), 129, 104, 91. HRMS (EI) m/z: [M] Calcd for $\mathrm{C}_{19} \mathrm{H}_{28} \mathrm{OF}_{2}: 310.2103$; Found: 310.2098 .<smiles>CC(=O)OCCCCC(F)(F)C(=O)CCCc1ccccc1</smiles>

5,5-Difluoro-6-oxo-9-phenylnonyl acetate (3I). The reaction was carried out using General Procedure A. Compound 31 (101 mg, 81\% yield) as a colorless oil was purified with silica gel chromatography (Petroleum ether: Ethyl acetate $=20: 1) .{ }^{1} \mathrm{H}$ NMR $\left(400 \mathrm{MHz}, \mathrm{CDCl}_{3}\right) \delta 7.29(\mathrm{t}, J=$ $7.2 \mathrm{~Hz}, 2 \mathrm{H}), 7.23-7.16(\mathrm{~m}, 3 \mathrm{H}), 4.06$ (t, $J=6.4 \mathrm{~Hz}, 2 \mathrm{H}), 2.72-2.62(\mathrm{~m}, 4 \mathrm{H}), 2.05(\mathrm{~s}, 3 \mathrm{H}), 2.02-$ $1.91(\mathrm{~m}, 4 \mathrm{H}), 1.73-1.63(\mathrm{~m}, 2 \mathrm{H}), 1.57-1.47(\mathrm{~m}, 2 \mathrm{H}) .{ }^{19} \mathrm{~F}$ NMR $\left(376 \mathrm{MHz}, \mathrm{CDCl}_{3}\right) \delta-107.4(\mathrm{t}, J=$ $17.3 \mathrm{~Hz}, 2 \mathrm{~F}) .{ }^{13} \mathrm{C} \mathrm{NMR}\left(126 \mathrm{MHz}, \mathrm{CDCl}_{3}\right) \delta 201.0(\mathrm{t}, J=31.9 \mathrm{~Hz}), 171.1,141.1,128.5,128.4,126.1$, $118.0(\mathrm{t}, J=252.4 \mathrm{~Hz}), 63.8,35.4,34.8,32.0(\mathrm{t}, J=22.9 \mathrm{~Hz}), 28.2,24.1,20.9,18.0(\mathrm{t}, J=4.3 \mathrm{~Hz})$. MS (EI): m/z (\%) 312 (M) , 292, 274, 147, 104 (100), 91. HRMS (EI) m/z: [M] Calcd for $\mathrm{C}_{17} \mathrm{H}_{22} \mathrm{O}_{3} \mathrm{~F}_{2}$ : 312.1537; Found: 312.1533. 
$\overbrace{0}^{Y_{0}^{F}}$ OTBS

8-((tert-Butyldimethylsilyl)oxy)-5,5-difluoro-1-phenyloctan-4-one (3m). The reaction was carried out using General Procedure A. Compound 3m (93 mg, 63\% yield) as a colorless oil was purified with silica gel chromatography (Petroleum ether: Ethyl acetate $=50: 1) .{ }^{1} \mathrm{H} \mathrm{NMR}\left(400 \mathrm{MHz}, \mathrm{CDCl}_{3}\right)$ $\delta 7.30(\mathrm{t}, J=7.2 \mathrm{~Hz}, 2 \mathrm{H}), 7.24-7.16(\mathrm{~m}, 3 \mathrm{H}), 3.64(\mathrm{t}, J=6.0 \mathrm{~Hz}, 2 \mathrm{H}), 2.72-2.61(\mathrm{~m}, 4 \mathrm{H}), 2.08-$ $1.92(\mathrm{~m}, 4 \mathrm{H}), 1.70-1.61(\mathrm{~m}, 2 \mathrm{H}), 0.90(\mathrm{~s}, 9 \mathrm{H}), 0.05(\mathrm{~s}, 6 \mathrm{H}) .{ }^{19} \mathrm{~F}$ NMR $\left(376 \mathrm{MHz}, \mathrm{CDCl}_{3}\right) \delta-107.3(\mathrm{t}$, $J=17.7 \mathrm{~Hz}, 2 \mathrm{~F}) .{ }^{13} \mathrm{C} \mathrm{NMR}\left(101 \mathrm{MHz}, \mathrm{CDCl}_{3}\right) \delta 201.0(\mathrm{t}, J=31.5 \mathrm{~Hz}), 141.1,128.42,128.40,126.1$, $118.4(\mathrm{t}, J=252.7 \mathrm{~Hz}), 62.0,35.5,34.8,29.2(\mathrm{t}, J=23.2 \mathrm{~Hz}), 25.9,24.6(\mathrm{t}, J=4.0 \mathrm{~Hz}), 24.2,18.3$, 5.4. MS (DART): $\mathrm{m} / \mathrm{z}(\%) 371(\mathrm{M}+\mathrm{H})^{+}$. HRMS (DART) $\mathrm{m} / \mathrm{z}:[\mathrm{M}+\mathrm{H}]^{+}$Calcd for $\mathrm{C}_{20} \mathrm{H}_{33} \mathrm{O}_{2} \mathrm{~F}_{2} \mathrm{Si}$ : 371.2212; Found: 371.2208.<smiles>O=C(CCCc1ccccc1)C(F)(F)CCCCCCl</smiles>

10-Chloro-5,5-difluoro-1-phenyldecan-4-one (3n). The reaction was carried out using General Procedure A. Compound 3n (75 mg, 62\% yield) as a yellow oil was purified with reverse-phase flash column chromatography $\left(\mathrm{CH}_{3} \mathrm{CN}: \mathrm{H}_{2} \mathrm{O}=8: 2\right) .{ }^{1} \mathrm{H} \mathrm{NMR}\left(400 \mathrm{MHz}, \mathrm{CDCl}_{3}\right) \delta 7.30(\mathrm{t}, J=7.2 \mathrm{~Hz}, 2 \mathrm{H})$, $7.24-7.17(\mathrm{~m}, 3 \mathrm{H}), 3.54(\mathrm{t}, J=6.8 \mathrm{~Hz}, 2 \mathrm{H}), 2.72-2.63(\mathrm{~m}, 4 \mathrm{H}), 2.05-1.90(\mathrm{~m}, 4 \mathrm{H}), 1.83-1.75$ $(\mathrm{m}, 2 \mathrm{H}), 1.55-1.42(\mathrm{~m}, 4 \mathrm{H}) .{ }^{19} \mathrm{~F}$ NMR $\left(376 \mathrm{MHz}, \mathrm{CDCl}_{3}\right) \delta-107.3(\mathrm{t}, J=17.3 \mathrm{~Hz}, 2 \mathrm{~F}) .{ }^{13} \mathrm{C}$ NMR $\left(126 \mathrm{MHz}, \mathrm{CDCl}_{3}\right) \delta 201.1(\mathrm{t}, J=31.5 \mathrm{~Hz}), 141.1,128.42,128.40,126.1,118.0(\mathrm{t}, J=252.3 \mathrm{~Hz}), 44.6$, 35.5, 34.7, 32.3 (t, $J=22.9 \mathrm{~Hz}), 32.1,26.4,24.1,20.6(\mathrm{t}, J=4.4 \mathrm{~Hz})$. MS (EI): m/z (\%) $302(\mathrm{M})^{+}, 147$, 129, 104 (100), 91. HRMS (EI) m/z: [M] Calcd for $\mathrm{C}_{16} \mathrm{H}_{21} \mathrm{OF}_{2} \mathrm{Cl}$ : 302.1249; Found: 302.1252.<smiles>N#CCCCCC(F)(F)C(=O)CCCc1ccccc1</smiles>

6,6-Difluoro-7-oxo-10-phenyldecanenitrile (3o). The reaction was carried out using General Procedure A. Compound 30 (55 mg, 49\% yield) as a yellow oil was purified with silica gel chromatography (Petroleum ether: Ethyl acetate = 10: 1). ${ }^{1} \mathrm{H}$ NMR $\left(400 \mathrm{MHz}, \mathrm{CDCl}_{3}\right) \delta 7.30(\mathrm{t}, J=$ $7.6 \mathrm{~Hz}, 2 \mathrm{H}), 7.23-7.15(\mathrm{~m}, 3 \mathrm{H}), 2.72-2.62(\mathrm{~m}, 4 \mathrm{H}), 2.37(\mathrm{t}, J=6.4 \mathrm{~Hz}, 2 \mathrm{H}), 2.07-1.92(\mathrm{~m}, 4 \mathrm{H})$, $1.77-1.68(\mathrm{~m}, 2 \mathrm{H}), 1.67-1.59(\mathrm{~m}, 2 \mathrm{H}) .{ }^{19} \mathrm{~F} \mathrm{NMR}\left(376 \mathrm{MHz}, \mathrm{CDCl}_{3}\right) \delta-107.2(\mathrm{t}, J=17.7 \mathrm{~Hz}, 2 \mathrm{~F})$. ${ }^{13} \mathrm{C} \mathrm{NMR}\left(126 \mathrm{MHz}, \mathrm{CDCl}_{3}\right) \delta 200.7(\mathrm{t}, J=31.9 \mathrm{~Hz}), 141.0,128.5,128.4,126.1,119.1,117.6(\mathrm{t}, J=$ 252.6 Hz), 35.3, 34.7, 31.5 (t, $J=23.4 \mathrm{~Hz}), 25.0,24.1,20.6$ (t, $J=4.4 \mathrm{~Hz}), 17.0$. MS (DART): m/z (\%) $297\left(\mathrm{M}+\mathrm{NH}_{4}\right)^{+}$. HRMS (DART) m/z: $\left[\mathrm{M}+\mathrm{NH}_{4}\right]^{+}$Calcd for $\mathrm{C}_{16} \mathrm{H}_{23} \mathrm{~N}_{2} \mathrm{OF}_{2}$ : 297.1773; Found: 


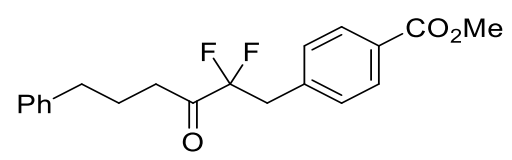

Methyl 4-(2,2-difluoro-3-oxo-6-phenylhexyl)benzoate (3p). The reaction was carried out using General Procedure A. Compound 3p (97 mg, 70\% yield) as a colorless oil was purified with silica gel chromatography (Petroleum ether: Ethyl acetate = 30: 1). ${ }^{1} \mathrm{H}$ NMR $\left(400 \mathrm{MHz}, \mathrm{CDCl}_{3}\right) \delta 7.98(\mathrm{~d}$, $J=8.0 \mathrm{~Hz}, 2 \mathrm{H}), 7.31(\mathrm{~d}, J=8.0 \mathrm{~Hz}, 2 \mathrm{H}), 7.26(\mathrm{t}, J=7.2 \mathrm{~Hz}, 2 \mathrm{H}), 7.18(\mathrm{t}, J=7.2 \mathrm{~Hz}, 1 \mathrm{H}), 7.10(\mathrm{~d}, J$ $=7.2 \mathrm{~Hz}, 2 \mathrm{H}), 3.91(\mathrm{~s}, 3 \mathrm{H}), 3.34(\mathrm{t}, J=16.8 \mathrm{~Hz}, 2 \mathrm{H}), 2.58-2.49(\mathrm{~m}, 4 \mathrm{H}), 1.90-1.81(\mathrm{~m}, 2 \mathrm{H}) .{ }^{19} \mathrm{~F}$ $\operatorname{NMR}\left(376 \mathrm{MHz}, \mathrm{CDCl}_{3}\right) \delta-105.2(\mathrm{t}, J=16.9 \mathrm{~Hz}, 2 \mathrm{~F}) .{ }^{13} \mathrm{C} \mathrm{NMR}\left(126 \mathrm{MHz}, \mathrm{CDCl}_{3}\right) \delta 200.8(\mathrm{t}, J=$ $31.0 \mathrm{~Hz}), 166.7,141.0,136.2(\mathrm{t}, J=4.2 \mathrm{~Hz}), 130.7,129.7,129.6,128.4,128.3,126.1,116.7(\mathrm{t}, J=$ $255.2 \mathrm{~Hz}), 52.1,39.0$ (t, $J=23.7 \mathrm{~Hz}), 36.0,34.6,23.9 . \mathrm{MS}(\mathrm{EI}): \mathrm{m} / \mathrm{z}(\%) 346(\mathrm{M})^{+}, 104(100) . \mathrm{HRMS}$ (EI) $\mathrm{m} / \mathrm{z}:[\mathrm{M}]^{+}$Calcd for $\mathrm{C}_{20} \mathrm{H}_{20} \mathrm{~F}_{2} \mathrm{O}_{3}$ : 346.1381; Found: 346.1377 .<smiles>O=C(OCCCC(F)(F)C(=O)CCCc1ccccc1)c1ccc(Cl)cc1</smiles>

4,4-Difluoro-5-oxo-8-phenyloctyl 4-chlorobenzoate. (3q). The reaction was carried out using General Procedure A. Compound 3q (139 mg, 88\% yield) as a yellow oil was purified with silica gel chromatography (Petroleum ether: Ethyl acetate $=30: 1) .{ }^{1} \mathrm{H} \mathrm{NMR}\left(400 \mathrm{MHz}, \mathrm{CDCl}_{3}\right) \delta 7.97(\mathrm{~d}, J=$ $8.8 \mathrm{~Hz}, 2 \mathrm{H}), 7.42(\mathrm{~d}, J=8.8 \mathrm{~Hz}, 2 \mathrm{H}), 7.29(\mathrm{t}, J=7.2 \mathrm{~Hz}, 2 \mathrm{H}), 7.23-7.15(\mathrm{~m}, 3 \mathrm{H}), 4.35(\mathrm{t}, J=6.4 \mathrm{~Hz}$, 2H), $2.71(\mathrm{t}, J=7.2 \mathrm{~Hz}, 2 \mathrm{H}), 2.65(\mathrm{t}, J=7.6 \mathrm{~Hz}, 2 \mathrm{H}), 2.20-2.06(\mathrm{~m}, 2 \mathrm{H}), 2.01-1.90(\mathrm{~m}, 4 \mathrm{H}) .{ }^{19} \mathrm{~F}$ $\operatorname{NMR}\left(376 \mathrm{MHz}, \mathrm{CDCl}_{3}\right) \delta-107.3(\mathrm{t}, J=17.3 \mathrm{~Hz}, 2 \mathrm{~F}) .{ }^{13} \mathrm{C} \mathrm{NMR}\left(126 \mathrm{MHz}, \mathrm{CDCl}_{3}\right) \delta 200.7(\mathrm{t}, J=$ $31.8 \mathrm{~Hz}), 165.5,141.0,139.5,130.9,128.8,128.5,128.4,126.1,117.7$ (t, $J=252.6 \mathrm{~Hz}), 64.1,35.3$, 34.8, $29.2(\mathrm{t}, J=23.6 \mathrm{~Hz}), 24.1,21.0(\mathrm{t}, J=4.2 \mathrm{~Hz}) . \mathrm{MS}(\mathrm{ESI}): \mathrm{m} / \mathrm{z}(\%) 417(\mathrm{M}+\mathrm{Na})^{+}$. HRMS (ESI) $\mathrm{m} / \mathrm{z}:[\mathrm{M}+\mathrm{Na}]^{+}$Calcd for $\mathrm{C}_{21} \mathrm{H}_{21} \mathrm{O}_{3} \mathrm{ClF}_{2} \mathrm{Na}$ : 417.1039; Found: 417.1040.<smiles>O=C(OCCCC(F)(F)C(=O)CCCc1ccccc1)c1ccccc1</smiles>

4,4-Difluoro-5-oxo-8-phenyloctyl benzoate (3r). The reaction was carried out using General Procedure A. Compound 3r (94 mg, 65\% yield) as a colorless oil was purified with silica gel chromatography (Petroleum ether: Ethyl acetate $=30: 1) .{ }^{1} \mathrm{H}$ NMR $\left(400 \mathrm{MHz}, \mathrm{CDCl}_{3}\right) \delta 8.06-8.01$ (m, 2H), 7.57 (t, $J=7.6 \mathrm{~Hz}, 1 \mathrm{H}), 7.45$ (t, $J=7.6 \mathrm{~Hz}, 2 \mathrm{H}), 7.29$ (t, $J=7.2 \mathrm{~Hz}, 2 \mathrm{H}), 7.23-7.15$ (m, 
$3 \mathrm{H}), 4.36(\mathrm{t}, J=6.4 \mathrm{~Hz}, 2 \mathrm{H}), 2.71(\mathrm{t}, J=7.2 \mathrm{~Hz}, 2 \mathrm{H}), 2.65(\mathrm{t}, J=7.2 \mathrm{~Hz}, 2 \mathrm{H}), 2.22-2.07(\mathrm{~m}, 2 \mathrm{H})$, $2.02-1.91(\mathrm{~m}, 4 \mathrm{H}) .{ }^{19} \mathrm{~F}$ NMR $\left(376 \mathrm{MHz}, \mathrm{CDCl}_{3}\right) \delta-107.3(\mathrm{t}, J=17.3 \mathrm{~Hz}, 2 \mathrm{~F}) .{ }^{13} \mathrm{C} \mathrm{NMR}(126 \mathrm{MHz}$, $\left.\mathrm{CDCl}_{3}\right) \delta 200.7(\mathrm{t}, J=31.6 \mathrm{~Hz}), 166.4,141.1,133.1,130.0,129.6,128.5,128.42,128.40,126.1,117.8$ (t, $J=252.5 \mathrm{~Hz}), 63.8,35.3,34.8,29.2(\mathrm{t}, J=23.2 \mathrm{~Hz}), 24.1,21.0(\mathrm{t}, J=4.4 \mathrm{~Hz}) . \mathrm{MS}(\mathrm{ESI}): \mathrm{m} / \mathrm{z}(\%)$ $383(\mathrm{M}+\mathrm{Na})^{+}$. HRMS (ESI) m/z: [M+Na] $]^{+}$Calcd for $\mathrm{C}_{21} \mathrm{H}_{22} \mathrm{O}_{3} \mathrm{~F}_{2} \mathrm{Na}$ : 383.1429; Found: 383.1427.

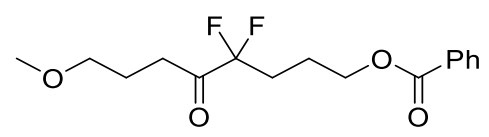

4,4-Difluoro-8-methoxy-5-oxooctyl benzoate (3s). The reaction was carried out using General Procedure A. Compound 3s (93 mg, 74\% yield) as a yellow oil was purified with silica gel chromatography (Petroleum ether: Ethyl acetate $=15: 1) .{ }^{1} \mathrm{H}$ NMR $\left(400 \mathrm{MHz}, \mathrm{CDCl}_{3}\right) \delta 8.02(\mathrm{~d}, J=$ $7.6 \mathrm{~Hz}, 2 \mathrm{H}), 7.55(\mathrm{t}, J=7.6 \mathrm{~Hz}, 1 \mathrm{H}), 7.43(\mathrm{t}, J=7.6 \mathrm{~Hz}, 2 \mathrm{H}), 4.35(\mathrm{t}, J=6.0 \mathrm{~Hz}, 2 \mathrm{H}), 3.38(\mathrm{t}, J=6.0$ $\mathrm{Hz}, 2 \mathrm{H}), 3.29(\mathrm{~s}, 3 \mathrm{H}), 2.78(\mathrm{t}, J=7.2 \mathrm{~Hz}, 2 \mathrm{H}), 2.23-2.08(\mathrm{~m}, 2 \mathrm{H}), 2.00-1.85(\mathrm{~m}, 4 \mathrm{H}) .{ }^{19} \mathrm{~F}$ NMR $\left(376 \mathrm{MHz}, \mathrm{CDCl}_{3}\right) \delta-107.5(\mathrm{t}, J=17.7 \mathrm{~Hz}, 2 \mathrm{~F}) .{ }^{13} \mathrm{C} \mathrm{NMR}\left(126 \mathrm{MHz}, \mathrm{CDCl}_{3}\right) \delta 200.7(\mathrm{t}, J=31.6 \mathrm{~Hz})$, 166.3, 133.0, 130.0, 129.5, 128.3, 117.8 (t, $J=252.6 \mathrm{~Hz}), 71.1,63.8,58.5,32.9,29.3$ (t, $J=23.3 \mathrm{~Hz})$, 22.8, $21.0(\mathrm{t}, J=4.5 \mathrm{~Hz})$. MS (EI): m/z (\%) 294 (M-HF) $)^{+}, 101$ (100). HRMS (EI) m/z: [M-HF] Calcd for $\mathrm{C}_{16} \mathrm{H}_{19} \mathrm{FO}_{4}$ : 294.1267; Found: 294.1279.<smiles>CC(C)(C)OCCCC(F)(F)C(=O)CCCC(=O)OC(C)(C)C</smiles>

9-(tert-Butoxy)-4,4-difluoro-5,9-dioxononyl benzoate (3t). The reaction was carried out using General Procedure A. Compound 3t (95 mg, 62\% yield) as a yellow oil was purified with silica gel chromatography (Petroleum ether: Ethyl acetate $=15: 1) .{ }^{1} \mathrm{H}$ NMR $\left(400 \mathrm{MHz}, \mathrm{CDCl}_{3}\right) \delta 8.01(\mathrm{~d}, J=$ $7.6 \mathrm{~Hz}, 2 \mathrm{H}), 7.54(\mathrm{t}, J=7.6 \mathrm{~Hz}, 1 \mathrm{H}), 7.42(\mathrm{t}, J=7.6 \mathrm{~Hz}, 2 \mathrm{H}), 4.34(\mathrm{t}, J=6.0 \mathrm{~Hz}, 2 \mathrm{H}), 2.75(\mathrm{t}, J=6.8$ $\mathrm{Hz}, 2 \mathrm{H}), 2.25(\mathrm{t}, J=6.8 \mathrm{~Hz}, 2 \mathrm{H}), 2.22-2.07(\mathrm{~m}, 2 \mathrm{H}), 1.99-1.84(\mathrm{~m}, 4 \mathrm{H}), 1.42(\mathrm{~s}, 9 \mathrm{H}) .{ }^{19} \mathrm{~F}$ NMR $\left(376 \mathrm{MHz}, \mathrm{CDCl}_{3}\right) \delta-107.4(\mathrm{t}, J=17.7 \mathrm{~Hz}, 2 \mathrm{~F}) .{ }^{13} \mathrm{C} \mathrm{NMR}\left(126 \mathrm{MHz}, \mathrm{CDCl}_{3}\right) \delta 200.2(\mathrm{t}, J=31.8 \mathrm{~Hz})$, 172.0, 166.3, 133.0, 130.0, 129.5, 128.3, 117.7 (t, $J=252.6 \mathrm{~Hz}), 80.4,63.7,35.0,34.0,29.2(\mathrm{t}, J=$ $23.3 \mathrm{~Hz}), 28.0,21.0(\mathrm{t}, J=4.4 \mathrm{~Hz}), 18.0 . \mathrm{MS}(\mathrm{EI}): \mathrm{m} / \mathrm{z}(\%) 328(\mathrm{M}-\mathrm{Bu}+\mathrm{H})^{+}, 105(100)$. HRMS (EI) $\mathrm{m} / \mathrm{z}:[\mathrm{M}-\mathrm{Bu}+\mathrm{H}]^{+}$Calcd for $\mathrm{C}_{16} \mathrm{H}_{18} \mathrm{~F}_{2} \mathrm{O}_{5}$ : 328.1122; Found: 328.1118 . 


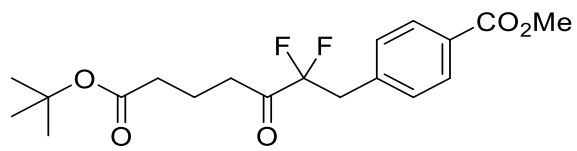

Methyl 4-(7-(tert-butoxy)-2,2-difluoro-3,7-dioxoheptyl)benzoate (3u). The reaction was carried out using General Procedure A. Compound 3u (96 mg, 65\% yield) as a yellow oil was purified with silica gel chromatography (Petroleum ether: Ethyl acetate = 30: 1). ${ }^{1} \mathrm{H}$ NMR $\left(400 \mathrm{MHz}, \mathrm{CDCl}_{3}\right) \delta 7.97(\mathrm{~d}$, $J=8.0 \mathrm{~Hz}, 2 \mathrm{H}), 7.30(\mathrm{~d}, J=8.0 \mathrm{~Hz}, 2 \mathrm{H}), 3.89(\mathrm{~s}, 3 \mathrm{H}), 3.34$ (t, $J=17.2 \mathrm{~Hz}, 2 \mathrm{H}), 2.58$ (t, $J=7.2 \mathrm{~Hz}$, 2H), $2.17(\mathrm{t}, J=7.2 \mathrm{~Hz}, 2 \mathrm{H}), 1.84-1.75(\mathrm{~m}, 2 \mathrm{H}), 1.40(\mathrm{~s}, 9 \mathrm{H}) .{ }^{19} \mathrm{~F}$ NMR $\left(376 \mathrm{MHz}, \mathrm{CDCl}_{3}\right) \delta-105.4$ $(\mathrm{t}, J=16.9 \mathrm{~Hz}, 2 \mathrm{~F}) .{ }^{13} \mathrm{C} \mathrm{NMR}\left(101 \mathrm{MHz}, \mathrm{CDCl}_{3}\right) \delta 200.4(\mathrm{t}, J=31.1 \mathrm{~Hz}), 172.0,166.6,136.1(\mathrm{t}, J=$ $3.7 \mathrm{~Hz}), 130.6,129.7,129.6,116.6(\mathrm{t}, J=255.5 \mathrm{~Hz}), 80.4,52.1,38.8$ (t, $J=23.7 \mathrm{~Hz}), 35.7,34.0,28.0$, 17.9. MS (DART): $\mathrm{m} / \mathrm{z}(\%) 388.19\left(\mathrm{M}+\mathrm{NH}_{4}\right)^{+}$. HRMS (DART) $\mathrm{m} / \mathrm{z}:\left[\mathrm{M}+\mathrm{NH}_{4}\right]^{+}$calcd for $\mathrm{C}_{19} \mathrm{H}_{28} \mathrm{O}_{5} \mathrm{NF}_{2}$ : 388.1930 ; Found: 388.1931 .<smiles>O=C(CCCc1ccccc1)C(F)(F)CO[Mg]</smiles>

1-((tert-Butyldimethylsilyl)oxy)-2,2-difluoro-6-phenylhexan-3-one (3v). The reaction was carried out using General Procedure A at $100{ }^{\circ} \mathrm{C}$, in which $\left[\mathrm{PdCl}\left(\mathrm{C}_{3} \mathrm{H}_{5}\right)\right]_{2}(2.5 \mathrm{~mol} \%)$ was used as the catalyst and $\mathrm{IPr} \cdot \mathrm{HCl}(10 \mathrm{~mol} \%)$ was used as the ligand. Compound 3v (62 mg, 45\% yield) as a yellow oil was purified with silica gel chromatography (Petroleum ether: Ethyl acetate $=50: 1) .{ }^{1} \mathrm{H} \mathrm{NMR}(300 \mathrm{MHz}$, $\left.\mathrm{CDCl}_{3}\right) \delta 7.34-7.26(\mathrm{~m}, 2 \mathrm{H}), 7.24-7.17(\mathrm{~m}, 3 \mathrm{H}), 3.96(\mathrm{t}, J=16.0 \mathrm{~Hz}, 2 \mathrm{H}), 2.73(\mathrm{t}, J=7.2 \mathrm{~Hz}, 2 \mathrm{H})$, $2.66(\mathrm{t}, J=7.2 \mathrm{~Hz}, 2 \mathrm{H}), 2.03-1.91(\mathrm{~m}, 2 \mathrm{H}), 0.87(\mathrm{~s}, 9 \mathrm{H}), 0.07(\mathrm{~s}, 6 \mathrm{H}) .{ }^{19} \mathrm{~F}$ NMR $\left(282 \mathrm{MHz}, \mathrm{CDCl}_{3}\right)$ $\delta-117.0(\mathrm{t}, J=12.4 \mathrm{~Hz}, 2 \mathrm{~F}) .{ }^{13} \mathrm{C} \mathrm{NMR}\left(101 \mathrm{MHz}, \mathrm{CDCl}_{3}\right) \delta 201.5(\mathrm{t}, J=28.1 \mathrm{~Hz}), 141.2,128.4,126.0$, $115.5(\mathrm{t}, J=256.2 \mathrm{~Hz}), 63.2(\mathrm{t}, J=31.1 \mathrm{~Hz}), 37.5,34.8,25.6,24.0,18.1,-5.7 . \mathrm{MS}(\mathrm{EI}): \mathrm{m} / \mathrm{z}(\%) 285$ $(\mathrm{M}-t \mathrm{Bu})^{+}, 91$ (100). HRMS (EI) m/z: [M- $\left.t \mathrm{Bu}\right]^{+}$Calcd for $\mathrm{C}_{18} \mathrm{H}_{28} \mathrm{~F}_{2} \mathrm{O}_{2} \mathrm{Si}$ : 285.1122; Found: 285.1130 .<smiles>CC(C)(C)OC(=O)CCCC(=O)C(F)(F)CO[GaH2]</smiles>

tert-Butyl-7-((tert-butyldimethylsilyl)oxy)-6,6-difluoro-5-oxoheptanoate $(3 \mathbf{w})$. The reaction was carried out using General Procedure A at $100{ }^{\circ} \mathrm{C}$, in which $\left[\mathrm{PdCl}\left(\mathrm{C}_{3} \mathrm{H}_{5}\right)\right]_{2}(2.5 \mathrm{~mol} \%)$ was used as the catalyst and $\mathrm{IPr} \cdot \mathrm{HCl}(10 \mathrm{~mol} \%)$ was used as the ligand. Compound $\mathbf{3 w}(92.4 \mathrm{mg}, 63 \%$ yield $)$ as a colorless oil was purified with silica gel chromatography (Petroleum ether: Ethyl acetate $=50: 1) .{ }^{1} \mathrm{H}$ NMR (400 MHz, $\left.\mathrm{CDCl}_{3}\right) \delta 3.94(\mathrm{t}, J=12.4 \mathrm{~Hz}, 2 \mathrm{H}), 2.75(\mathrm{t}, J=7.2 \mathrm{~Hz}, 2 \mathrm{H}), 2.25$ (t, J=7.2 Hz, 2H), $1.93-1.84(\mathrm{~m}, 2 \mathrm{H}), 1.43(\mathrm{~s}, 9 \mathrm{H}), 0.86(\mathrm{~s}, 9 \mathrm{H}), 0.06(\mathrm{~s}, 6 \mathrm{H}) .{ }^{19} \mathrm{~F} \mathrm{NMR}\left(376 \mathrm{MHz}, \mathrm{CDCl}_{3}\right) \delta-116.7(\mathrm{t}$, 
$J=12.4 \mathrm{~Hz}, 2 \mathrm{~F}) .{ }^{13} \mathrm{C} \mathrm{NMR}\left(126 \mathrm{MHz}, \mathrm{CDCl}_{3}\right) \delta 201.0(\mathrm{t}, J=28.7 \mathrm{~Hz}), 172.1,115.5(\mathrm{t}, J=255.3 \mathrm{~Hz})$, 80.4, $63.1(\mathrm{t}, J=31.2 \mathrm{~Hz}), 37.2,34.2,28.1,25.6,18.2,17.9,-5.7 . \mathrm{MS}(\mathrm{EI}): \mathrm{m} / \mathrm{z}(\%) 293\left(\mathrm{M}-\mathrm{OC}_{4} \mathrm{H}_{9}\right)^{+}$, 253, 235, 193 (100), 159, 115, 57. HRMS (EI) m/z: [M-OC $\left.{ }_{4} \mathrm{H}_{9}\right]^{+}$Calcd for $\mathrm{C}_{13} \mathrm{H}_{23} \mathrm{O}_{3} \mathrm{~F}_{2} \mathrm{Si}$ : 293.1379; Found: 293.1377.

\section{Characterization data for compounds 7.}<smiles>CCOC(=O)C(F)(F)C(=O)CCCCc1ccccc1</smiles>

$N, N$-Diethyl-2,2-difluoro-3-oxo-6-phenylhexanamide (7a). The reaction was carried out using General Procedure B. Compound 7a (100 mg, 84\% yield) as a yellow oil was purified with silica gel chromatography (Petroleum ether: Ethyl acetate $=10: 1) .{ }^{1} \mathrm{H}$ NMR $\left(400 \mathrm{MHz}, \mathrm{CDCl}_{3}\right) \delta 7.31-7.25$ $(\mathrm{m}, 2 \mathrm{H}), 7.23-7.15(\mathrm{~m}, 3 \mathrm{H}), 3.47(\mathrm{q}, J=7.0 \mathrm{~Hz}, 2 \mathrm{H}), 3.38(\mathrm{q}, J=7.1 \mathrm{~Hz}, 2 \mathrm{H}), 2.72(\mathrm{t}, J=7.2 \mathrm{~Hz}$, 2H), $2.67(\mathrm{t}, J=7.7 \mathrm{~Hz}, 2 \mathrm{H}), 2.06-1.93(\mathrm{~m}, 2 \mathrm{H}), 1.23(\mathrm{t}, J=7.1 \mathrm{~Hz}, 3 \mathrm{H}), 1.16(\mathrm{t}, J=7.1 \mathrm{~Hz}, 3 \mathrm{H})$. ${ }^{19} \mathrm{~F} \mathrm{NMR}\left(376 \mathrm{MHz}, \mathrm{CDCl}_{3}\right) \delta-109.7$ (s, 2F). ${ }^{13} \mathrm{C} \mathrm{NMR}\left(126 \mathrm{MHz}, \mathrm{CDCl}_{3}\right) \delta 197.6(\mathrm{t}, J=27.2 \mathrm{~Hz})$, $160.9(\mathrm{t}, J=27.1 \mathrm{~Hz}), 141.3,128.4,128.4,126.0,110.8(\mathrm{t}, J=269.3 \mathrm{~Hz}), 41.5(\mathrm{t}, J=5.2 \mathrm{~Hz}), 41.2$, 36.6, 34.8, 24.4, 14.2, 12.2. MS (EI): m/z (\%) $269(\mathrm{M}-\mathrm{Et}+\mathrm{H})^{+}, 147$ (100). HRMS (EI) m/z: [M] $]^{+}$Calcd for $\mathrm{C}_{16} \mathrm{H}_{21} \mathrm{NO}_{2} \mathrm{~F}_{2}$ : 297.1540; Found: 297.1535.

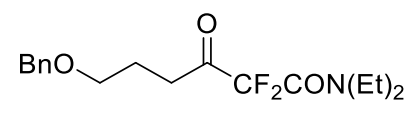

6-(Benzyloxy)- $N, N$-diethyl-2,2-difluoro-3-oxohexanamide (7b). The reaction was carried out using General Procedure B. Compound $7 \mathbf{b}$ The product (102 mg, $78 \%$ yield) as a yellow oil was purified with silica gel chromatography (Petroleum ether: Ethyl acetate $=10: 1) .{ }^{1} \mathrm{H} \mathrm{NMR}\left(400 \mathrm{MHz}, \mathrm{CDCl}_{3}\right)$ $\delta 7.36-7.24(\mathrm{~m}, 5 \mathrm{H}), 4.49$ (s, 2H), $3.50(\mathrm{t}, J=6.1 \mathrm{~Hz}, 2 \mathrm{H}), 3.45(\mathrm{q}, J=7.1 \mathrm{~Hz}, 2 \mathrm{H}), 3.38(\mathrm{q}, J=7.1$ $\mathrm{Hz}, 2 \mathrm{H}), 2.83(\mathrm{t}, J=7.1 \mathrm{~Hz}, 2 \mathrm{H}), 2.06-1.94(\mathrm{~m}, 2 \mathrm{H}), 1.23(\mathrm{t}, J=7.1 \mathrm{~Hz}, 3 \mathrm{H}), 1.16(\mathrm{t}, J=7.1 \mathrm{~Hz}$, 3H). ${ }^{19} \mathrm{~F} \mathrm{NMR}\left(376 \mathrm{MHz}, \mathrm{CDCl}_{3}\right) \delta-109.7(\mathrm{~s}, 2 \mathrm{~F}) .{ }^{13} \mathrm{C} \mathrm{NMR}\left(126 \mathrm{MHz}, \mathrm{CDCl}_{3}\right) \delta 197.6(\mathrm{t}, J=27.2$ Hz), $160.9(\mathrm{t}, J=27.1 \mathrm{~Hz}), 138.4,128.3,127.6,127.5,110.8(\mathrm{t}, J=268.7 \mathrm{~Hz}), 72.9,68.9,41.5(\mathrm{t}, J=$ $5.2 \mathrm{~Hz}), 41.1,34.2,23.2,14.2,12.2$. MS (EI): m/z (\%) 307 (M-HF) ${ }^{+}$, 91(100). HRMS (EI) m/z: [M$\mathrm{HF}]^{+}$Calcd for $\mathrm{C}_{17} \mathrm{H}_{22} \mathrm{NO}_{3} \mathrm{~F}: 307.1584$; Found: 307.1582 .<smiles>CCOC(=O)C(F)(F)C(=O)CCCC(=O)OC(C)(C)C</smiles>

tert-Butyl-7-(diethylamino)-6,6-difluoro-5,7-dioxoheptanoate (7c). The reaction was carried out using General Procedure B. Compound 7c (84 mg, 65\% yield) as a yellow oil was purified with silica 
gel chromatography (Petroleum ether: Ethyl acetate = 10: 1). ${ }^{1} \mathrm{H}$ NMR $\left(400 \mathrm{MHz}, \mathrm{CDCl}_{3}\right) \delta 3.46(\mathrm{q}$, $J=7.0 \mathrm{~Hz}, 2 \mathrm{H}), 3.36(\mathrm{q}, J=7.1 \mathrm{~Hz}, 2 \mathrm{H}), 2.74(\mathrm{t}, J=7.1 \mathrm{~Hz}, 2 \mathrm{H}), 2.27$ (t, $J=7.3 \mathrm{~Hz}, 2 \mathrm{H}), 1.99-1.85$ $(\mathrm{m}, 2 \mathrm{H}), 1.42(\mathrm{~s}, 9 \mathrm{H}), 1.21(\mathrm{t}, J=7.0 \mathrm{~Hz}, 3 \mathrm{H}), 1.14(\mathrm{t}, J=7.1 \mathrm{~Hz}, 3 \mathrm{H}) .{ }^{19} \mathrm{~F} \mathrm{NMR}\left(376 \mathrm{MHz}, \mathrm{CDCl}_{3}\right)$ $\delta$-109.9 (s, 2F). ${ }^{13} \mathrm{C} \mathrm{NMR}\left(126 \mathrm{MHz}, \mathrm{CDCl}_{3}\right) \delta 197.1(\mathrm{t}, J=27.3 \mathrm{~Hz}), 172.2,160.9(\mathrm{t}, J=26.9 \mathrm{~Hz})$, $110.7(\mathrm{t}, J=268.9 \mathrm{~Hz}), 80.3,41.4(\mathrm{t}, J=5.2 \mathrm{~Hz}), 41.1,36.3,34.1,28.0,18.3,14.1,12.2 . \mathrm{MS}(\mathrm{EI}): \mathrm{m} / \mathrm{z}$ (\%) $265(\mathrm{M}-t \mathrm{Bu}+\mathrm{H})^{+}, 248(100)$. HRMS (EI) m/z: $[\mathrm{M}-t \mathrm{Bu}+\mathrm{H}]^{+}$Calcd for $\mathrm{C}_{11} \mathrm{H}_{17} \mathrm{NO}_{4} \mathrm{~F}_{2}$ : 265.1126; Found: 265.1120.<smiles>CCOC(=O)C(F)(F)C(=O)CCCCCCCOC(=O)c1c(F)cc(F)cc1F</smiles>

9-(Diethylamino)-8,8-difluoro-7,9-dioxononyl 2,4,6-trifluorobenzoate (7d). The reaction was carried out using General Procedure B. Compound 7d (110 mg, 63\% yield) as a yellow oil was purified with silica gel chromatography (Petroleum ether: Ethyl acetate $=10: 1) .{ }^{1} \mathrm{H} \mathrm{NMR}(400 \mathrm{MHz}$, $\left.\mathrm{CDCl}_{3}\right) \delta 6.71(\mathrm{t}, J=8.4 \mathrm{~Hz}, 2 \mathrm{H}), 4.33(\mathrm{t}, J=6.4 \mathrm{~Hz}, 2 \mathrm{H}), 3.48(\mathrm{q}, J=7.2 \mathrm{~Hz}, 2 \mathrm{H}), 3.38(\mathrm{q}, J=7.2$ $\mathrm{Hz}, 2 \mathrm{H}), 2.70(\mathrm{t}, J=7.2 \mathrm{~Hz}, 2 \mathrm{H}), 1.79-1.60(\mathrm{~m}, 4 \mathrm{H}), 1.45-1.39(\mathrm{~m}, 4 \mathrm{H}), 1.23(\mathrm{t}, J=7.2 \mathrm{~Hz}, 3 \mathrm{H})$, $1.15(\mathrm{t}, J=7.2 \mathrm{~Hz}, 3 \mathrm{H}) .{ }^{19} \mathrm{~F}$ NMR $\left(376 \mathrm{MHz} \mathrm{CDCl}_{3}\right) \delta-102.2(\mathrm{~m}, 1 \mathrm{~F}),-106.4(\mathrm{t}, J=8.4 \mathrm{~Hz}, 2 \mathrm{~F}),-$ $109.9(\mathrm{~s}, 2 \mathrm{~F}) .{ }^{13} \mathrm{C} \mathrm{NMR}\left(126 \mathrm{MHz}, \mathrm{CDCl}_{3}\right) \delta 197.8(\mathrm{t}, J=27.1 \mathrm{~Hz}), 164.1(\mathrm{dt}, J=255.3 \mathrm{~Hz}, 15.4 \mathrm{~Hz})$, $161.6(\mathrm{ddd}, J=258.7 \mathrm{~Hz}, 15.2 \mathrm{~Hz}, 8.9 \mathrm{~Hz}), 160.9$ (t, $J=27.0 \mathrm{~Hz}), 160.9,110.8$ (t, $J=269.3 \mathrm{~Hz}), 107.9$ (td, $J=18.0 \mathrm{~Hz}, 4.9 \mathrm{~Hz}), 101.0(\mathrm{td}, J=26.1 \mathrm{~Hz}, 3.8 \mathrm{~Hz}), 66.0,41.5$ (t, $J=5.0 \mathrm{~Hz}), 41.1,37.2,28.3$, 28.2, 25.6, 22.6, 14.2, 12.2. MS (EI): m/z (\%) $437(\mathrm{M})^{+}, 159$ (100). HRMS (EI) m/z: [M] Calcd for $\mathrm{C}_{20} \mathrm{H}_{24} \mathrm{NO}_{4} \mathrm{~F}_{5}$ : 437.1625; Found: 437.1621.<smiles>CCOC(=O)C(F)(F)C(=O)CCCCCCOC(=O)c1scnc1C</smiles>

9-(Diethylamino)-8,8-difluoro-7,9-dioxononyl-4-methylthiazole-5-carboxylate (7e). The reaction was carried out using General Procedure B. Compound 7 e (97 $\mathrm{mg}, 60 \%$ yield) as a yellow oil was purified with silica gel chromatography (Petroleum ether: Ethyl acetate $=10: 1) .{ }^{1} \mathrm{H} \mathrm{NMR}(400 \mathrm{MHz}$, $\left.\mathrm{CDCl}_{3}\right) \delta 8.76(\mathrm{~s}, 1 \mathrm{H}), 4.27(\mathrm{t}, J=6.4 \mathrm{~Hz}, 2 \mathrm{H}), 3.48(\mathrm{q}, J=7.2 \mathrm{~Hz}, 2 \mathrm{H}), 3.38(\mathrm{q}, J=7.2 \mathrm{~Hz}, 2 \mathrm{H}), 2.76$ (s, 3H), 2.70 (t, $J=7.2 \mathrm{~Hz}, 2 \mathrm{H}), 1.72(\mathrm{~m}, 4 \mathrm{H}), 1.51-1.33(\mathrm{~m}, 4 \mathrm{H}), 1.23$ (t, $J=7.2 \mathrm{~Hz}, 3 \mathrm{H}), 1.15$ (t, $J$ $=7.2 \mathrm{~Hz}, 3 \mathrm{H}) .{ }^{19} \mathrm{~F} \mathrm{NMR}\left(376 \mathrm{MHz}, \mathrm{CDCl}_{3}\right) \delta-109.8(\mathrm{~s}, 2 \mathrm{~F}) .{ }^{13} \mathrm{C} \mathrm{NMR}\left(101 \mathrm{MHz}, \mathrm{CDCl}_{3}\right) \delta 197.7(\mathrm{t}$, $J=26.9 \mathrm{~Hz}), 162.1,160.9(\mathrm{t}, J=26.8 \mathrm{~Hz}), 160.5,155.2,122.3,110.8(\mathrm{t}, J=268.8 \mathrm{~Hz}), 65.3,41.5(\mathrm{t}$, 
$J=5.2 \mathrm{~Hz}), 41.1,37.2,28.39,28.36,25.7,22.6,17.3,14.2,12.2 . \mathrm{MS}(\mathrm{EI}): \mathrm{m} / \mathrm{z}(\%) 404(\mathrm{M})^{+}, 126$ (100). HRMS (EI) m/z: [M] $]^{+}$Calcd for $\mathrm{C}_{18} \mathrm{H}_{26} \mathrm{~N}_{2} \mathrm{O}_{4} \mathrm{~F}_{2} \mathrm{~S}: 404.1581$; Found: 404.1582.<smiles>CCOC(=O)CCCCCCOC(=O)C(C)NC(=O)OCc1ccccc1</smiles>

(S)-9-(Diethylamino)-8,8-difluoro-7,9-dioxononyl 2-((tert-butoxycarbonyl)amino)propanoate (7f). The reaction was carried out using General Procedure B. Compound $7 \mathbf{f}$ ( $85 \mathrm{mg}, 47 \%$ yield) as a yellow oil was purified with silica gel chromatography (Petroleum ether: Ethyl acetate $=5: 1$ ). ${ }^{1} \mathrm{H}$ NMR (400 MHz, $\left.\mathrm{CDCl}_{3}\right) \delta 5.09(\mathrm{~d}, J=4.0 \mathrm{~Hz}, 1 \mathrm{H}), 4.30-4.20(\mathrm{~m}, 1 \mathrm{H}), 4.11-4.04(\mathrm{~m}, 2 \mathrm{H}), 3.44$ (q, $J=7.2 \mathrm{~Hz}, 2 \mathrm{H}), 3.34$ (q, $J=7.2 \mathrm{~Hz}, 2 \mathrm{H}), 2.65(\mathrm{t}, J=7.2 \mathrm{~Hz}, 2 \mathrm{H}), 1.70-1.59$ (m, 4H), 1.39 (s, 9H), $1.34-1.32(\mathrm{~m}, 7 \mathrm{H}), 1.22(\mathrm{t}, J=7.2 \mathrm{~Hz}, 3 \mathrm{H}), 1.14(\mathrm{t}, J=7.2 \mathrm{~Hz}, 3 \mathrm{H}) .{ }^{19} \mathrm{~F} \mathrm{NMR}\left(376 \mathrm{MHz}, \mathrm{CDCl}_{3}\right) \delta$ -109.9 (s, 2F). ${ }^{13} \mathrm{C}$ NMR (101 MHz, $\left.\mathrm{CDCl}_{3}\right) \delta 197.6(\mathrm{t}, J=27.3 \mathrm{~Hz}), 173.3,160.9$ (t, $\left.J=27.3 \mathrm{~Hz}\right)$, 155.0, $110.8(\mathrm{t}, J=270.7 \mathrm{~Hz}), 79.6,65.1,49.2,41.4(\mathrm{t}, J=5.1 \mathrm{~Hz}), 41.1,37.1,28.3,28.2,25.5,22.6$, 18.6, 14.1, 12.2. MS (DART): m/z (\%) 451(M+H) ${ }^{+}$. HRMS (DART) m/z: $[\mathrm{M}+\mathrm{H}]^{+}$calcd for $\mathrm{C}_{21} \mathrm{H}_{37} \mathrm{O}_{6} \mathrm{~F}_{2} \mathrm{~N}_{2}$ : 451.2614; Found: 451.2613 .<smiles>CCCCNC(=O)C(F)(F)C(=O)CCCc1ccccc1</smiles>

$N$-Butyl-2,2-difluoro-3-oxo-6-phenylhexanamide (7g). The reaction was carried out using General Procedure B. Compound $7 \mathrm{~g}(62 \mathrm{mg}, 52 \%$ yield) as a yellow oil was purified with silica gel chromatography (Petroleum ether: Ethyl acetate = 10: 1). ${ }^{1} \mathrm{H} \mathrm{NMR}\left(400 \mathrm{MHz}, \mathrm{CDCl}_{3}\right) \delta 7.25-7.30$ $(\mathrm{m}, 2 \mathrm{H}), 7.15-7.20(\mathrm{~m}, 3 \mathrm{H}), 6.45(\mathrm{~s}, 1 \mathrm{H}), 3.31(\mathrm{q}, J=6.8 \mathrm{~Hz}, 2 \mathrm{H}), 2.82(\mathrm{t}, J=6.8 \mathrm{~Hz}, 2 \mathrm{H}), 2.63(\mathrm{t}, J$ $=7.6 \mathrm{~Hz}, 2 \mathrm{H}), 2.05-1.84(\mathrm{~m}, 2 \mathrm{H}), 1.59-1.47(\mathrm{~m}, 2 \mathrm{H}), 1.29-1.38(\mathrm{~m}, 2 \mathrm{H}), 0.92(\mathrm{t}, J=7.2 \mathrm{~Hz}, 3 \mathrm{H})$. ${ }^{19} \mathrm{~F} \mathrm{NMR}\left(376 \mathrm{MHz}, \mathrm{CDCl}_{3}\right) \delta-115.2(\mathrm{~s}, 2 \mathrm{~F}) .{ }^{13} \mathrm{C} \mathrm{NMR}\left(101 \mathrm{MHz}, \mathrm{CDCl}_{3}\right) \delta 198.9(\mathrm{t}, J=27.4 \mathrm{~Hz})$, $161.2(\mathrm{t}, J=26.4 \mathrm{~Hz}), 141.1,128.6,128.5,126.2,109.36(\mathrm{t}, J=265.5 \mathrm{~Hz}), 39.6,37.1,34.7,31.2,24.1$, 20.0, 13.8. MS (DART): m/z (\%) $298(\mathrm{M}+\mathrm{H})^{+}$. HRMS (DART) m/z: $[\mathrm{M}+\mathrm{H}]^{+}$calcd for $\mathrm{C}_{16} \mathrm{H}_{22} \mathrm{O}_{2} \mathrm{~F}_{2} \mathrm{~N}$ : 298.1613; Found: 298.1616.<smiles>CCOC(=O)C(=O)CCCCCCOC(=O)c1c(F)cc(F)cc1F</smiles>

9-Ethoxy-8,8-difluoro-7,9-dioxononyl-2,4,6-trifluorobenzoate (7h). The reaction was carried out using General Procedure B. Compound 7 h $(66 \mathrm{mg}, 40 \%$ yield) as a yellow oil was purified with 
silica gel chromatography (Petroleum ether: Ethyl acetate $=10: 1) .{ }^{1} \mathrm{H}$ NMR $\left(400 \mathrm{MHz}, \mathrm{CDCl}_{3}\right) \delta 6.72$ $(\mathrm{t}, J=8.4 \mathrm{~Hz}, 2 \mathrm{H}), 4.36(\mathrm{q}, J=7.2 \mathrm{~Hz}, 2 \mathrm{H}), 4.33(\mathrm{t}, J=6.4 \mathrm{~Hz}, 2 \mathrm{H}), 2.74(\mathrm{t}, J=7.2 \mathrm{~Hz}, 2 \mathrm{H}), 1.77-$ $1.70(\mathrm{~m}, 2 \mathrm{H}), 1.69-1.60(\mathrm{~m}, 2 \mathrm{H}), 1.47-1.37(\mathrm{~m}, 4 \mathrm{H}), 1.34(\mathrm{t}, J=7.2 \mathrm{~Hz}, 3 \mathrm{H}) .{ }^{19} \mathrm{~F}$ NMR $(376 \mathrm{MHz}$, $\left.\mathrm{CDCl}_{3}\right) \delta-102.1(\mathrm{~m}, 1 \mathrm{~F}),-106.4(\mathrm{t}, J=8.6 \mathrm{~Hz}, 2 \mathrm{~F}),-113.9(\mathrm{~s}, 2 \mathrm{~F}) .{ }^{13} \mathrm{C} \mathrm{NMR}\left(101 \mathrm{MHz}, \mathrm{CDCl}_{3}\right) \delta$ 197.3 (t, $J=28.2 \mathrm{~Hz}), 164.1(\mathrm{dt}, J=261.0 \mathrm{~Hz}, 15.6 \mathrm{~Hz}), 161.4(\mathrm{ddd}, J=259.4 \mathrm{~Hz}, 15.5 \mathrm{~Hz}, 8.7 \mathrm{~Hz}$ ), $161.4(\mathrm{t}, J=30.8 \mathrm{~Hz}), 160.9,108.1(\mathrm{t}, J=262.9 \mathrm{~Hz}), 107.8(\mathrm{td}, J=18.4 \mathrm{~Hz}, 4.6 \mathrm{~Hz}), 101.1(\mathrm{td}, J=$ $26.3 \mathrm{~Hz}, 3.7 \mathrm{~Hz}), 65.9,63.7,36.4,28.2,28.2,25.5,22.2,13.8$. MS (DART): m/z (\%) $411.1(\mathrm{M}+\mathrm{H})^{+}$. HRMS (DART) m/z: [M+H] $]^{+}$calcd for $\mathrm{C}_{18} \mathrm{H}_{20} \mathrm{O}_{5} \mathrm{~F}_{5}$ : 411.1225; Found: 411.1227.

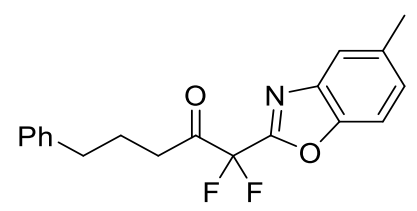

1,1-Difluoro-1-(5-methylbenzo[ $d]$ oxazol-2-yl)-5-phenylpentan-2-one (7i). The reaction was carried out using General Procedure B. Compound 7i (60 mg, 45\% yield) as a yellow oil was purified with silica gel chromatography (Petroleum ether: Ethyl acetate $=20: 1) .{ }^{1} \mathrm{H}$ NMR $\left(400 \mathrm{MHz}, \mathrm{CDCl}_{3}\right) \delta 7.60$ $(\mathrm{s}, 1 \mathrm{H}), 7.50(\mathrm{~d}, J=8.4 \mathrm{~Hz}, 1 \mathrm{H}), 7.32-7.23(\mathrm{~m}, 3 \mathrm{H}), 7.22-7.15(\mathrm{~m}, 3 \mathrm{H}), 2.91(\mathrm{t}, J=6.8 \mathrm{~Hz}, 2 \mathrm{H})$, $2.69(\mathrm{t}, J=7.6 \mathrm{~Hz}, 2 \mathrm{H}), 2.50(\mathrm{~s}, 3 \mathrm{H}), 2.10-2.01(\mathrm{~m}, 2 \mathrm{H}) .{ }^{19} \mathrm{~F}$ NMR $\left(376 \mathrm{MHz}, \mathrm{CDCl}_{3}\right) \delta-108.0(\mathrm{~s}$, 2F). ${ }^{13} \mathrm{C} \mathrm{NMR}\left(126 \mathrm{MHz}, \mathrm{CDCl}_{3}\right) \delta 196.3(\mathrm{t}, J=28.4 \mathrm{~Hz}), 154.6(\mathrm{t}, J=32.3 \mathrm{~Hz}), 149.0,140.9,140.0$, 135.7, 128.6, 128.5, 128.4, 126.1, 121.1, 110.9, 109.3 (t, $J=256.0 \mathrm{~Hz}), 36.1,34.6,24.2$, 21.5. MS (EI): m/z (\%) $329(\mathrm{M})^{+}, 91$ (100). HRMS (EI) m/z: [M] ${ }^{+}$Calcd for $\mathrm{C}_{19} \mathrm{H}_{17} \mathrm{NO}_{2} \mathrm{~F}_{2}$ : 329.1227; Found: 329.1224.

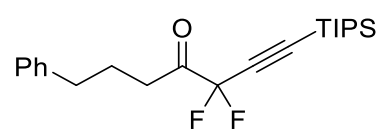

3,3-Difluoro-7-phenyl-1-(triisopropylsilyl)hept-1-yn-4-one (7j). The reaction was carried out using General Procedure B. $\mathrm{P}(\mathrm{o}-\mathrm{MePh})_{3}(10 \mathrm{~mol} \%)$ was used as the ligand. Compound $7 \mathbf{j}$ (59 mg, 52\% yield, $0.3 \mathrm{mmol}$ scale) as a yellow oil was purified with silica gel chromatography (Petroleum ether: Ethyl acetate = 20: 1). ${ }^{1} \mathrm{H} \mathrm{NMR}\left(400 \mathrm{MHz}, \mathrm{CDCl}_{3}\right) \delta 7.32-7.28(\mathrm{~m}, 2 \mathrm{H}), 7.23-7.17(\mathrm{~m}, 3 \mathrm{H}), 2.77$ $(\mathrm{t}, J=7.2 \mathrm{~Hz}, 2 \mathrm{H}), 2.67(\mathrm{t}, J=7.5 \mathrm{~Hz}, 2 \mathrm{H}), 2.09-1.95(\mathrm{~m}, 2 \mathrm{H}), 1.09(\mathrm{~m}, 21 \mathrm{H}) .{ }^{19} \mathrm{~F} \mathrm{NMR}(376 \mathrm{MHz}$, $\left.\mathrm{CDCl}_{3}\right) \delta-94.4(\mathrm{~s}, 2 \mathrm{~F}) .{ }^{13} \mathrm{C} \mathrm{NMR}\left(126 \mathrm{MHz}, \mathrm{CDCl}_{3}\right) \delta 195.5(\mathrm{t}, J=30.5 \mathrm{~Hz}), 140.9,128.44,128.36$, 126.1, 106.4 (t, $J=245.2 \mathrm{~Hz}), 96.5$ (t, $J=5.5 \mathrm{~Hz}), 95.0$ (t, $J=36.9 \mathrm{~Hz}), 34.9,34.7,24.5,18.3,10.8$. MS (DART): m/z (\%) $396\left(\mathrm{M}+\mathrm{NH}_{4}\right)^{+}$. HRMS (DART) m/z: $\left[\mathrm{M}+\mathrm{NH}_{4}\right]^{+}$calcd for $\mathrm{C}_{22} \mathrm{H}_{36} \mathrm{ONF}_{2} \mathrm{Si}$ : 378.2190; Found: 396.2530. 


\section{Synthesis of Compounds 9 and 11.}

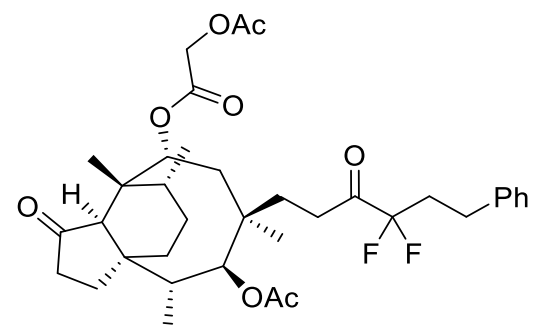

$(3 \mathrm{a} R, 4 R, 5 R, 7 R, 8 S, 9 R, 9 \mathrm{a} R, 12 R)-8$-Acetoxy-7-(4,4-difluoro-3-oxo-6-phenylhexyl)-4,7,9,12tetramethyl-3-oxodecahydro-4,9a-propanocyclopenta[8]annulen-5-yl 2-acetoxyacetate (9). Compound 9 (186.3 $\mathrm{mg}, 72 \%$ yield) as a brown oil was purified with silica gel chromatography $\left(\right.$ Petroleum ether: Ethyl acetate = 10: 1). ${ }^{1} \mathrm{H}$ NMR $\left(400 \mathrm{MHz}, \mathrm{CDCl}_{3}\right) \delta 7.29-7.14(\mathrm{~m}, 5 \mathrm{H}), 5.53(\mathrm{~d}$, $J=8.0 \mathrm{~Hz}, 1 \mathrm{H}), 4.83(\mathrm{~d}, J=6.0 \mathrm{~Hz}, 1 \mathrm{H}), 4.36(\mathrm{~s}, 2 \mathrm{H}), 2.82-2.75(\mathrm{~m}, 2 \mathrm{H}), 2.66-2.42(\mathrm{~m}, 3 \mathrm{H}), 2.38$ $-2.10(\mathrm{~m}, 6 \mathrm{H}), 2.06(\mathrm{~s}, 3 \mathrm{H}), 1.97(\mathrm{~s}, 3 \mathrm{H}), 1.95-1.80(\mathrm{~m}, 3 \mathrm{H}), 1.76-1.57(\mathrm{~m}, 2 \mathrm{H}), 1.53-1.44(\mathrm{~m}$, $1 \mathrm{H}), 1.42(\mathrm{~s}, 3 \mathrm{H}), 1.37-1.22(\mathrm{~m}, 3 \mathrm{H}), 1.15-1.04(\mathrm{~m}, 1 \mathrm{H}), 0.81(\mathrm{~s}, 3 \mathrm{H}), 0.79(\mathrm{~s}, 3 \mathrm{H}), 0.67(\mathrm{~d}, J=6.8$ $\mathrm{Hz}, 3 \mathrm{H}) .{ }^{19} \mathrm{~F} \mathrm{NMR}\left(376 \mathrm{MHz}, \mathrm{CDCl}_{3}\right) \delta-106.8(\mathrm{dt}, J=270.7 \mathrm{~Hz}, 15.0 \mathrm{~Hz}, 1 \mathrm{~F}),-108.3(\mathrm{dt}, J=270.7$ $\mathrm{Hz}, 15.0 \mathrm{~Hz}, 1 \mathrm{~F}) .{ }^{13} \mathrm{C} \mathrm{NMR}\left(101 \mathrm{MHz}, \mathrm{CDCl}_{3}\right) \delta 216.5,200.8(\mathrm{t}, J=31.3 \mathrm{~Hz}), 170.2,167.0,140.0$, $128.4,128.2,126.2,117.8(\mathrm{t}, J=253.5 \mathrm{~Hz}), 77.2,69.4,61.3,58.0,45.1,41.6,40.5,39.3,36.3,34.4(\mathrm{t}$, $J=23.2 \mathrm{~Hz}), 34.3,30.8,30.0,27.4$ (t, $J=5.1 \mathrm{~Hz}), 26.8,25.8,24.6,21.6,20.6,20.0,16.5,14.5,11.6$. MS (DART): m/z (\%) $664\left(\mathrm{M}+\mathrm{NH}_{4}\right)^{+}$. HRMS (DART) $\mathrm{m} / \mathrm{z}:\left[\mathrm{M}+\mathrm{NH}_{4}\right]^{+}$Calcd for $\mathrm{C}_{36} \mathrm{H}_{52} \mathrm{O}_{8} \mathrm{NF}_{2}$ : 664.3656; Found: 664.3641.

Gram-scale synthesis of 9: To a $500 \mathrm{~mL}$ of Schlenk tube, $\mathrm{PdCl}_{2}\left(\mathrm{PPh}_{3}\right)_{2}(140.4 \mathrm{mg}, 0.2 \mathrm{mmol}, 0.05$ equiv), XantPhos (231.4 mg, $0.4 \mathrm{mmol}, 0.1$ equiv) and $\mathrm{K}_{3} \mathrm{PO}_{4}(2.55 \mathrm{~g}, 12 \mathrm{mmol}, 3.0$ equiv) were added. The reaction mixture was then evacuated and backfilled with Ar (3 times). The mixture was evacuated again and backfilled with carbon monoxide $\mathrm{CO}(1 \mathrm{~atm})$, followed by addition of alkyl-9-BBN (4.0 mmol, 1.0 equiv), difluoroalkyl iodide $2 \mathrm{a}$ (2.26 g, $8.0 \mathrm{mmol}, 2.0$ equiv), $\mathrm{H}_{2} \mathrm{O}(100.8 \mu \mathrm{L}, 5.6 \mathrm{mmol}$, 1.4 equiv) and dioxane $(20 \mathrm{~mL})$. The tube was screw-capped and heated to $60{ }^{\circ} \mathrm{C}$ (oil bath). After stirring for $24 \mathrm{~h}$, the reaction mixture was cooled to room temperature, the reaction mixture was diluted with ethyl acetate and $\mathrm{H}_{2} \mathrm{O}$. The resulting mixture was extracted with ethyl acetate, the combined organic layers were washed with brine, dried over $\mathrm{Na}_{2} \mathrm{SO}_{4}$, filtered and concentrated. The residue was purified with silica gel chromatography to give the product $9(1.55 \mathrm{~g}, 60 \%)$. 


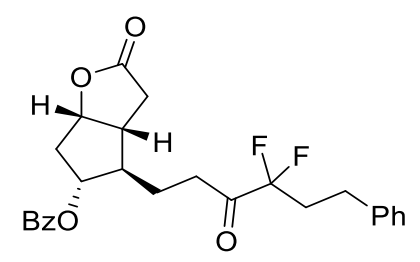

(3aR,4R,5R,6aS)-4-(4,4-Difluoro-3-oxo-6-phenylhexyl)-2-oxohexahydro-2H-

cyclopenta[b]furan-5-yl benzoate (11). Compound $11(103.3 \mathrm{mg}, 62 \%$ yield) as a yellow oil was purified with silica gel chromatography (Petroleum ether: Ethyl acetate $=5: 1) .{ }^{1} \mathrm{H} \mathrm{NMR}(400 \mathrm{MHz}$, $\left.\mathrm{CDCl}_{3}\right) \delta 7.97(\mathrm{~d}, J=7.2 \mathrm{~Hz}, 2 \mathrm{H}), 7.55(\mathrm{t}, J=7.2 \mathrm{~Hz}, 1 \mathrm{H}), 7.43(\mathrm{t}, J=7.6 \mathrm{~Hz}, 2 \mathrm{H}), 7.29$ (t, $J=7.2$ $\mathrm{Hz}, 2 \mathrm{H}), 7.24-7.15(\mathrm{~m}, 3 \mathrm{H}), 5.20-5.15(\mathrm{~m}, 1 \mathrm{H}), 5.08(\mathrm{t}, J=6.0 \mathrm{~Hz}, 1 \mathrm{H}), 2.95-2.84(\mathrm{~m}, 3 \mathrm{H}), 2.81$ $-2.75(\mathrm{~m}, 2 \mathrm{H}), 2.70-2.61(\mathrm{~m}, 1 \mathrm{H}), 2.53-2.40(\mathrm{~m}, 2 \mathrm{H}), 2.39-2.24(\mathrm{~m}, 3 \mathrm{H}), 2.15-2.07(\mathrm{~m}, 1 \mathrm{H})$, $1.77-1.59(\mathrm{~m}, 2 \mathrm{H}) .{ }^{19} \mathrm{~F} \mathrm{NMR}\left(376 \mathrm{MHz}, \mathrm{CDCl}_{3}\right) \delta-107.3(\mathrm{t}, J=15.0 \mathrm{~Hz}, 2 \mathrm{~F}) .{ }^{13} \mathrm{C}$ NMR $(101 \mathrm{MHz}$, $\left.\mathrm{CDCl}_{3}\right) \delta 200.1(\mathrm{t}, J=31.3 \mathrm{~Hz}), 176.4,165.9,139.6,133.2,129.5,129.4,128.5,128.4,128.2,126.3$, 117.7 (t, $J=253.5 \mathrm{~Hz}), 84.0,79.6,51.8,43.5,37.5,35.9,34.14$ (t, $J=23.2 \mathrm{~Hz}), 34.10,27.4$ (t, $J=5.1$ Hz), 25.7. MS (EI): m/z (\%) $456(\mathrm{M})^{+}, 334,270,230,105$ (100), 91, 77. HRMS (FI) m/z: [M] Calcd for $\mathrm{C}_{26} \mathrm{H}_{26} \mathrm{O}_{5} \mathrm{~F}_{2}$ : 456.1743; Found: 456.1747.

\section{Transformations of compound 3a to compounds 12-14.}<smiles>O=C(CCCc1ccccc1)C(F)(F)CCc1ccccc1</smiles>

$3 \mathbf{a}$

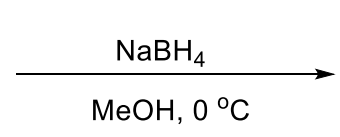

$\mathrm{MeOH}, 0^{\circ} \mathrm{C}$

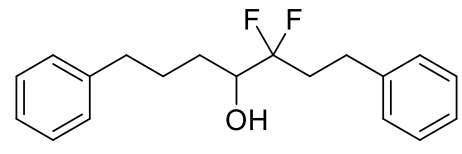

$12,99 \%$

Procedure: To a $25 \mathrm{~mL}$ of round bottomed flask were added compound $\mathbf{3 a}(0.3 \mathrm{mmol}, 1.0$ equiv) and methanol $(5 \mathrm{~mL}) . \mathrm{NaBH}_{4}\left(0.3 \mathrm{mmol}, 1.0\right.$ equiv) was then added at $0{ }^{\circ} \mathrm{C}$. After stirring for $1 \mathrm{~h}$ at $0{ }^{\circ} \mathrm{C}$, the reaction mixture was quenched by diluted hydrochloric acid, and diluted with DCM and $\mathrm{H}_{2} \mathrm{O}$. The resulting mixture was extracted with DCM for three times, the combined organic layers were dried over $\mathrm{Na}_{2} \mathrm{SO}_{4}$, filtered and concentrated to give a colorless oil 12 (90 mg, 99\% yield).

3,3-Difluoro-1,7-diphenylheptan-4-ol (12). ${ }^{1} \mathrm{H}$ NMR (400 MHz, $\left.\mathrm{CDCl}_{3}\right) \delta 7.36-7.29$ (m, 4H), 7.27 - $7.19(\mathrm{~m}, 6 \mathrm{H}), 3.82-3.71(\mathrm{~m}, 1 \mathrm{H}), 2.94-2.79(\mathrm{~m}, 2 \mathrm{H}), 2.75-2.61(\mathrm{~m}, 2 \mathrm{H}), 2.37-2.06(\mathrm{~m}, 2 \mathrm{H})$, $2.02-1.88(\mathrm{~m}, 2 \mathrm{H}), 1.80-1.67(\mathrm{~m}, 2 \mathrm{H}), 1.60-1.48(\mathrm{~m}, 1 \mathrm{H}) .{ }^{19} \mathrm{~F} \mathrm{NMR}\left(376 \mathrm{MHz}, \mathrm{CDCl}_{3}\right) \delta-110.4$ $(\mathrm{dm}, J=248.2 \mathrm{~Hz}, 1 \mathrm{~F}),-112.9(\mathrm{dm}, J=248.2 \mathrm{~Hz}, 1 \mathrm{~F}) .{ }^{13} \mathrm{C} \mathrm{NMR}\left(101 \mathrm{MHz}, \mathrm{CDCl}_{3}\right) \delta 141.9,140.7$, 128.5, 128.4, 128.3, 126.4, 125.9, $123.8(\mathrm{t}, J=246.4 \mathrm{~Hz}), 73.1(\mathrm{t}, J=29.3 \mathrm{~Hz}), 35.6,34.1(\mathrm{t}, J=24.2$ $\mathrm{Hz}), 29.6(\mathrm{t}, J=2.0 \mathrm{~Hz}), 27.6$ (t, $J=5.1 \mathrm{~Hz}), 27.4$. MS (EI): m/z (\%) $304(\mathrm{M})^{+}, 286,162,131(100)$, 104, 91. HRMS (EI) m/z: [M] Calcd for $\mathrm{C}_{19} \mathrm{H}_{22} \mathrm{OF}_{2}$ : 304.1633; Found: 304.1637. 

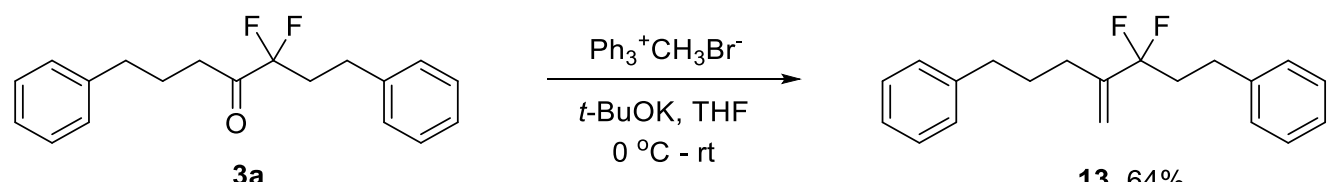

Procedure: To a $25 \mathrm{~mL}$ of Schlenck tube were added $\mathrm{Ph}_{3} \mathrm{P}^{+} \mathrm{CH}_{3} \mathrm{Br}^{-}$(0.36 mmol, 1.2 equiv), $t \mathrm{BuOK}$ (0.36 mmol, 1.2 equiv) and THF $(3 \mathrm{~mL})$. The resulting solution was stirred for $0.5 \mathrm{~h}$ at $0{ }^{\circ} \mathrm{C} .3 \mathbf{a}(0.3$ mmol, 1.0 equiv) was then added dropwise at the same temperature. After stirring for $8 \mathrm{~h}$ at room temperature, the reaction mixture was quenched by $\mathrm{H}_{2} \mathrm{O}$, and extracted with EtOAc for 3 times. The combined organic layers were washed with brine, dried over $\mathrm{Na}_{2} \mathrm{SO}_{4}$, and filtered. The filtrate was concentrated and the residue was purified with silica gel chromatography (Petroleum ether: Ethyl acetate $=70: 1)$ to give compound $13(57.5 \mathrm{mg}, 64 \%$ yield $)$ as a colorless oil.

(3,3-Difluoro-4-methyleneheptane-1,7-diyl)dibenzene (13). ${ }^{1} \mathrm{H}$ NMR $\left(400 \mathrm{MHz}, \mathrm{CDCl}_{3}\right) \delta 7.34-$ $7.28(\mathrm{~m}, 4 \mathrm{H}), 7.25-7.16(\mathrm{~m}, 6 \mathrm{H}), 5.46(\mathrm{~s}, 1 \mathrm{H}), 5.18(\mathrm{~s}, 1 \mathrm{H}), 2.80-2.73(\mathrm{~m}, 2 \mathrm{H}), 2.68(\mathrm{t}, J=7.6 \mathrm{~Hz}$, 2H), $2.31-2.15(\mathrm{~m}, 4 \mathrm{H}), 1.87(\mathrm{~m}, 2 \mathrm{H}) .{ }^{19} \mathrm{~F} \mathrm{NMR}\left(376 \mathrm{MHz}, \mathrm{CDCl}_{3}\right) \delta-99.4(\mathrm{t}, J=15.0 \mathrm{~Hz}, 2 \mathrm{~F}) .{ }^{13} \mathrm{C}$ NMR $\left(126 \mathrm{MHz}, \mathrm{CDCl}_{3}\right) \delta 144.1(\mathrm{t}, J=23.1 \mathrm{~Hz}), 141.9,140.6,128.5,128.39,128.36,128.3,126.2$, 125.9, 122.6 (t, $J=242.8 \mathrm{~Hz}), 114.2(\mathrm{t}, J=8.8 \mathrm{~Hz}), 37.9$ (t, $J=26.8 \mathrm{~Hz}), 35.5,29.8,29.5,28.6(\mathrm{t}, J=$ 4.4 Hz). MS (EI): m/z (\%) $300(\mathrm{M})^{+}, 145,104$ (100), 91, 65. HRMS (EI) m/z: [M] ${ }^{+}$Calcd for $\mathrm{C}_{20} \mathrm{H}_{22} \mathrm{~F}_{2}$ : 300.1684; Found: 300.1682.
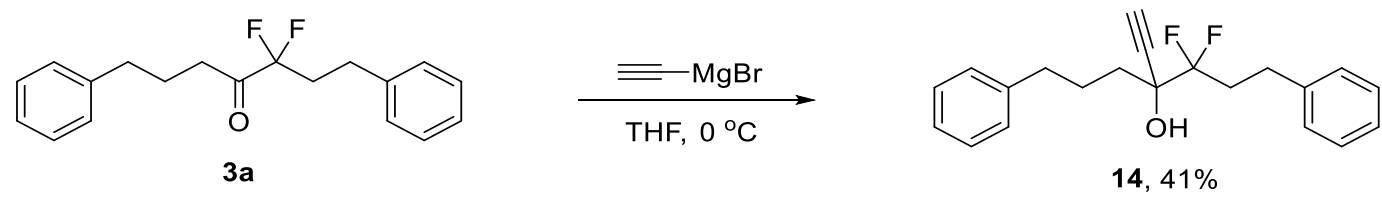

Procedure: To a $25 \mathrm{~mL}$ of Schlenck tube were added $3 \mathbf{a}(0.3 \mathrm{mmol}, 1.0$ equiv) and THF ( $2 \mathrm{~mL})$. The resulting solution was stirred at $0{ }^{\circ} \mathrm{C}$. Ethynylmagnesium bromide $(0.45 \mathrm{mmol}, 1.5$ equiv) was then added dropwise at the same temperature. After stirring for $10 \mathrm{~h}$ at $0{ }^{\circ} \mathrm{C}$, the reaction mixture was quenched by $\mathrm{H}_{2} \mathrm{O}$, and extracted with EtOAc for 3 times. The combined organic layers were washed with brine, dried over $\mathrm{Na}_{2} \mathrm{SO}_{4}$, and filtered. The filtrate was concentrated and the residue was purified with silica gel chromatography (Petroleum ether: Ethyl acetate $=15: 1)$ to give compound 14 (40.5 $\mathrm{mg}, 41 \%$ yield) as a colorless oil.

4-Ethynyl-3,3-difluoro-1,7-diphenylheptan-4-ol (14). ${ }^{1} \mathrm{H} \mathrm{NMR}\left(400 \mathrm{MHz}, \mathrm{CDCl}_{3}\right) \delta 7.36-7.29$ (m, 4H), $7.28-7.19(\mathrm{~m}, 6 \mathrm{H}), 2.93(\mathrm{t}, J=8.4 \mathrm{~Hz}, 2 \mathrm{H}), 2.72(\mathrm{t}, J=6.8 \mathrm{~Hz}, 2 \mathrm{H}), 2.56(\mathrm{~s}, 1 \mathrm{H}), 2.47-2.31$ $(\mathrm{m}, 3 \mathrm{H}), 2.08-1.96(\mathrm{~m}, 2 \mathrm{H}), 1.88-1.82(\mathrm{~m}, 2 \mathrm{H}) .{ }^{19} \mathrm{~F}$ NMR $\left(376 \mathrm{MHz}, \mathrm{CDCl}_{3}\right) \delta-112.9(\mathrm{dm}, J=$ 
$224.4 \mathrm{~Hz}, 1 \mathrm{~F}),-114.9(\mathrm{dm}, J=224.4 \mathrm{~Hz}, 1 \mathrm{~F}) .{ }^{13} \mathrm{C} \mathrm{NMR}\left(101 \mathrm{MHz}, \mathrm{CDCl}_{3}\right) \delta 141.8,140.7,128.5$, 128.4, 126.2, 125.9, 125.2, 122.7 (t, $J=252.5 \mathrm{~Hz}), 81.2,75.3,73.9$ (t, $J=28.3 \mathrm{~Hz}), 35.7,34.4,33.5$ (t, $J=24.2 \mathrm{~Hz}), 27.4(\mathrm{t}, J=4.0 \mathrm{~Hz}), 25.4 . \mathrm{MS}(\mathrm{EI}): \mathrm{m} / \mathrm{z}(\%) 328(\mathrm{M})^{+}, 310,290,206,173,155,104$ (100), 91. HRMS (FI) m/z: [M] Calcd for $\mathrm{C}_{21} \mathrm{H}_{22} \mathrm{OF}_{2}$ : 328.1633; Found: 328.1638.

\section{References.}

(1) Saito, B.; Fu, G. C. J. Am. Chem. Soc. 2008, 130, 6694.

(2) Levin, V. V.; Zemtsov, A. A.; Struchkova, M. I.; Dilman, A. D. Org. Lett. 2013, 15, 917.

(3) Xiao, Y.-L.; Min, Q.-Q.; Xu, C.; Wang, R.-W.; Zhang, X. Angew. Chem. Int. Ed. 2016, 55, 5837.

(4) Ge, S.-Z.; Hartwig, J. F. J. Am. Chem. Soc. 2014, 136, 14401.

(5) Zhang, M.; Li, W.; Duan, Y.; Xu, P.; Zhang, S.; Zhu, C. Org. Lett. 2016, 18, 3266.

(6) Dolbier Jr, W. R.; Burkholder, C. R.; Médebielle, M. J. Fluorine Chem. 1999, 95, 127.

(7) Guo, W.-H.; Luo, Z.-J.; Zeng, W.; Zhang, X. ACS Catal. 2017, 7, 896. 
11. Copies of ${ }^{1} \mathrm{H}$ NMR, ${ }^{19} \mathrm{~F}$ NMR and ${ }^{13} \mathrm{C}$ NMR spectra of compounds $2-3,7,9,11-14,16$. (3,3-Difluoro-3-iodopropyl)benzene (2a).
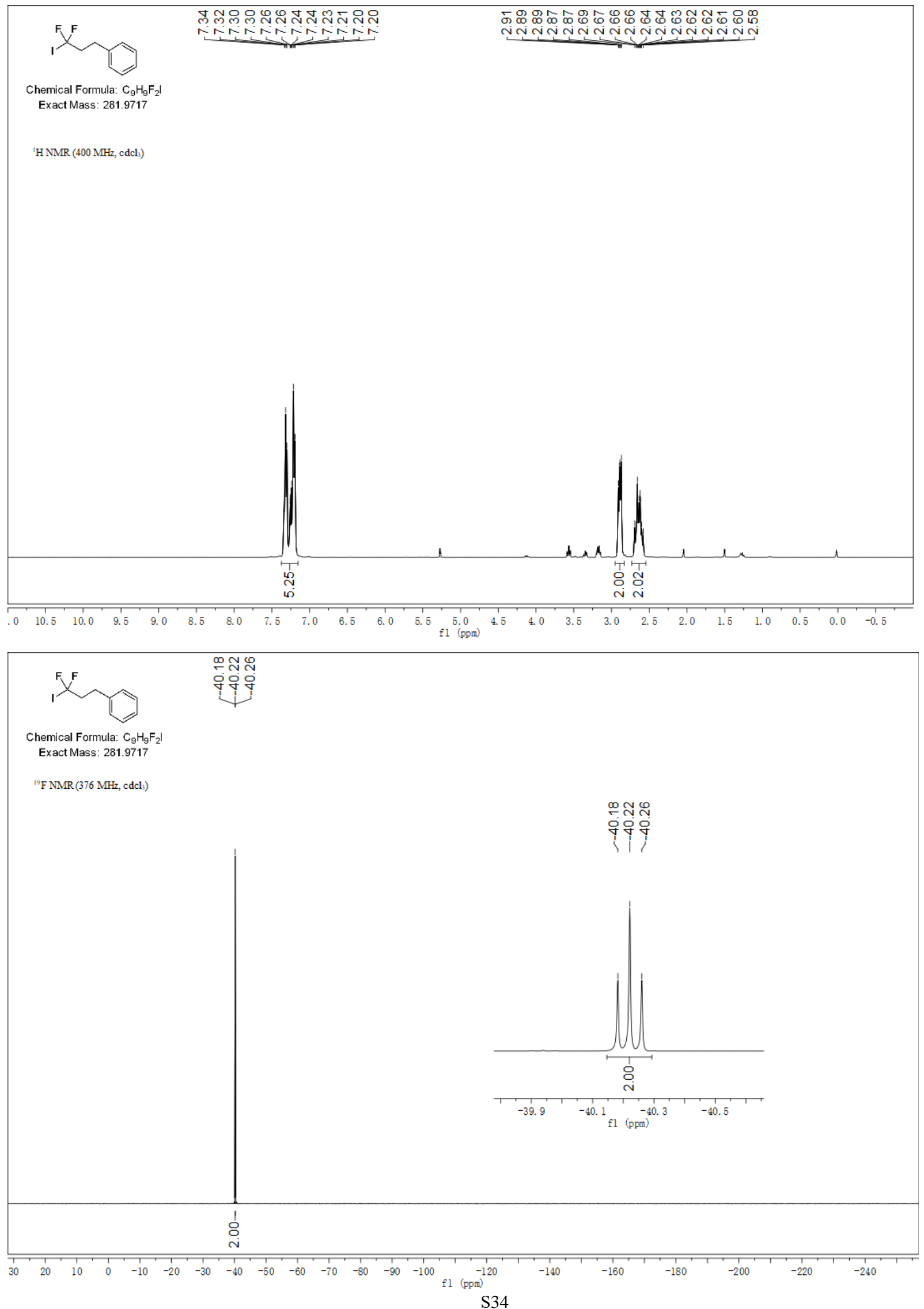


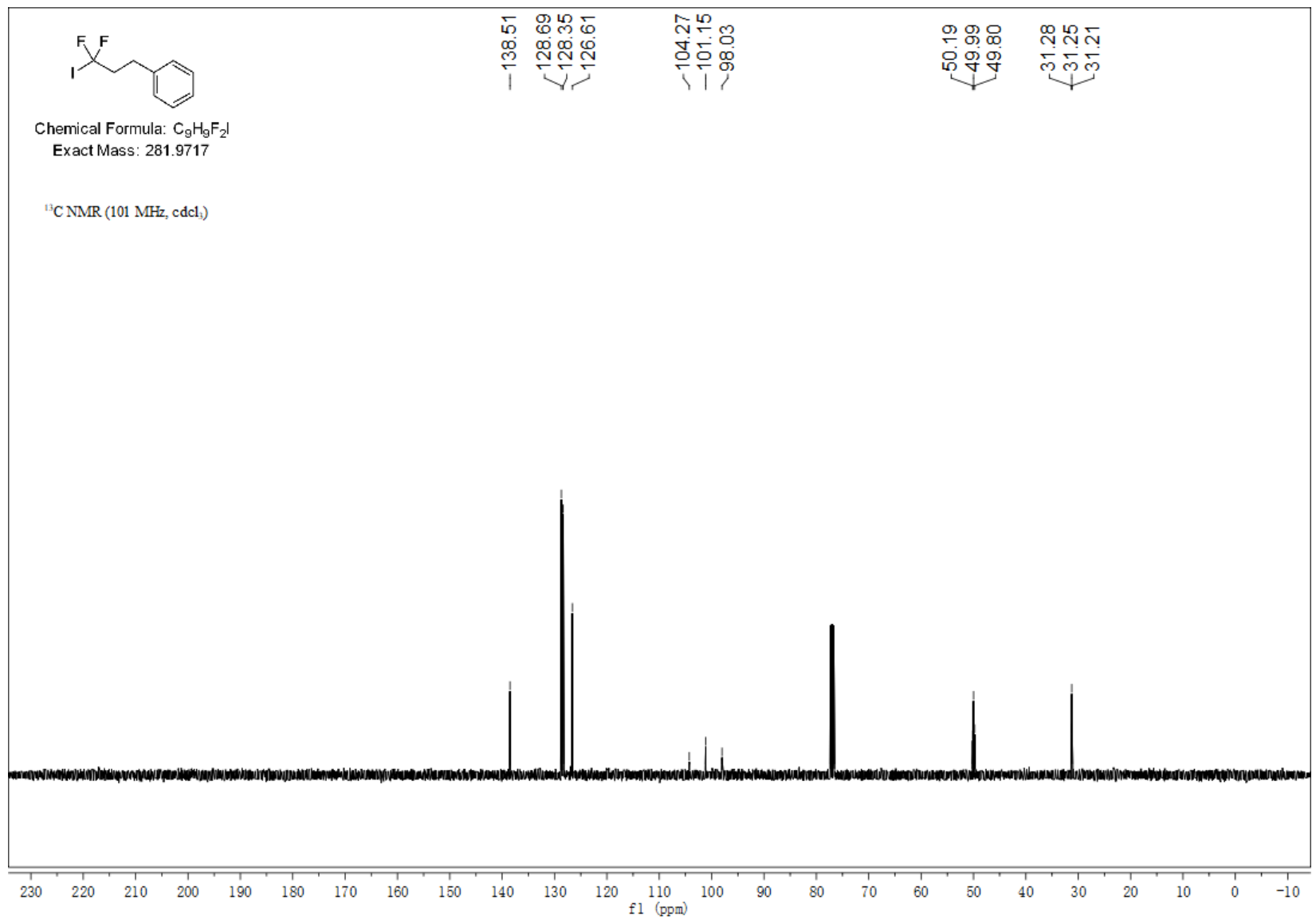

\section{1,1-Difluoro-1-iodononane (2b).}

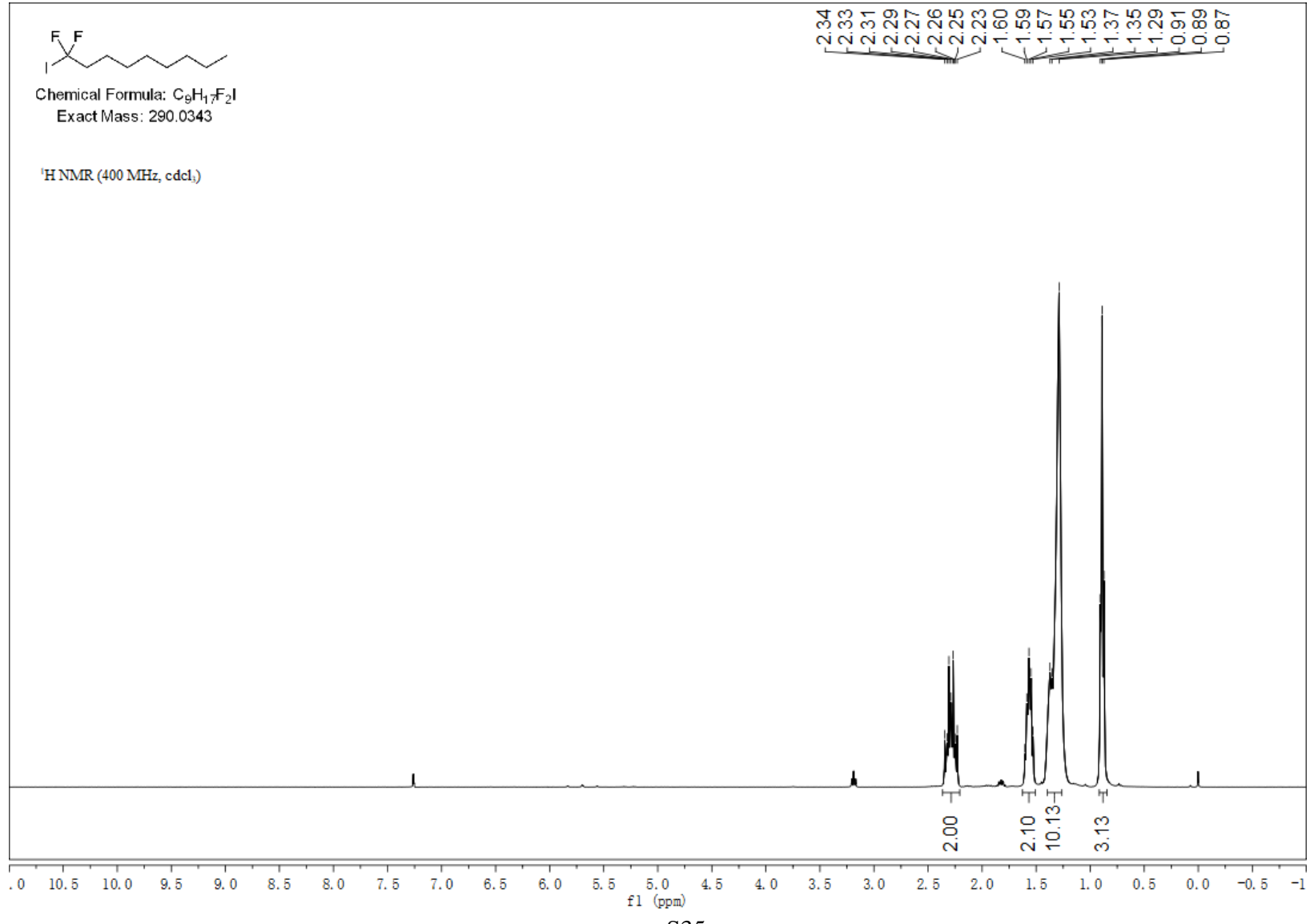




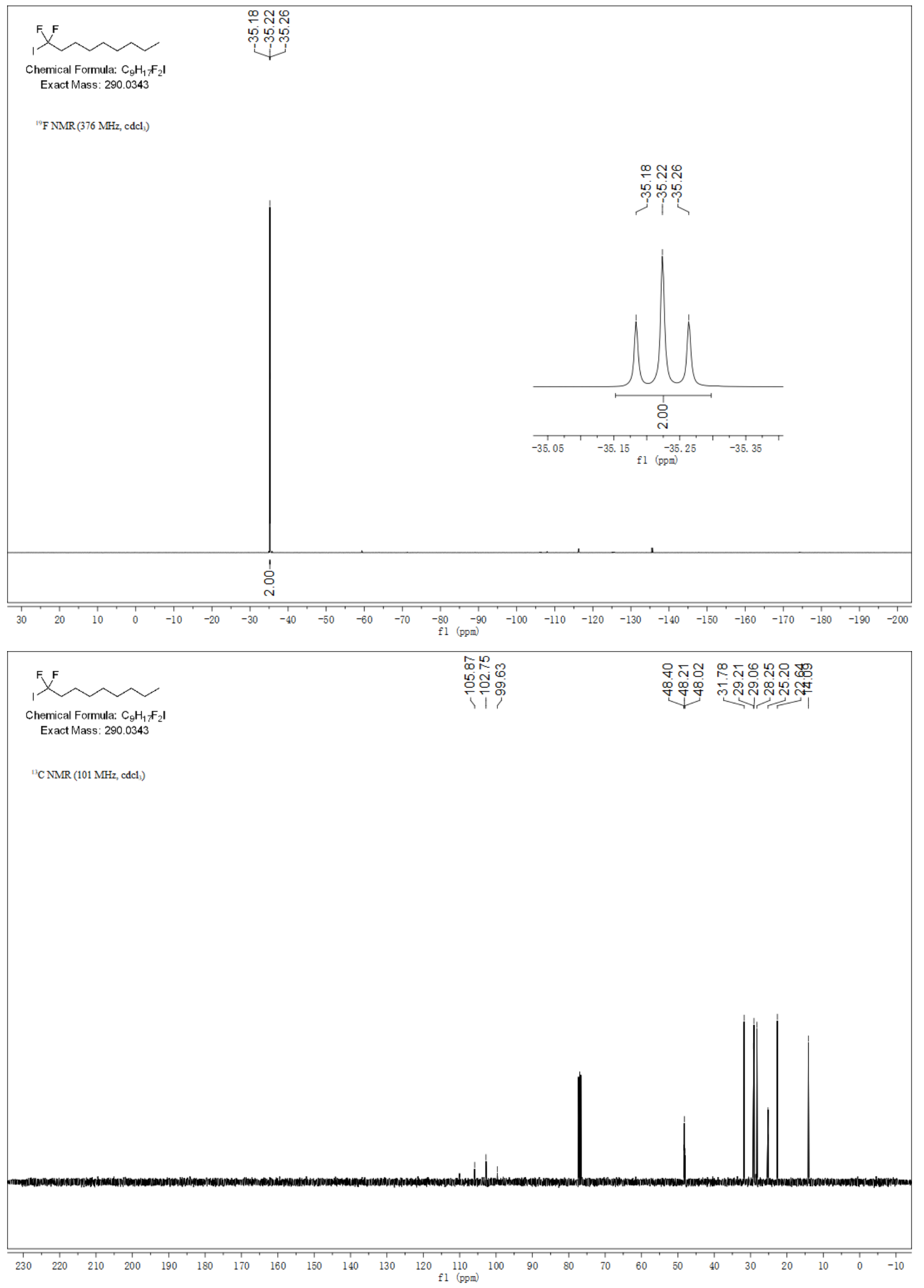


5,5-Difluoro-5-iodopentyl acetate (2c).
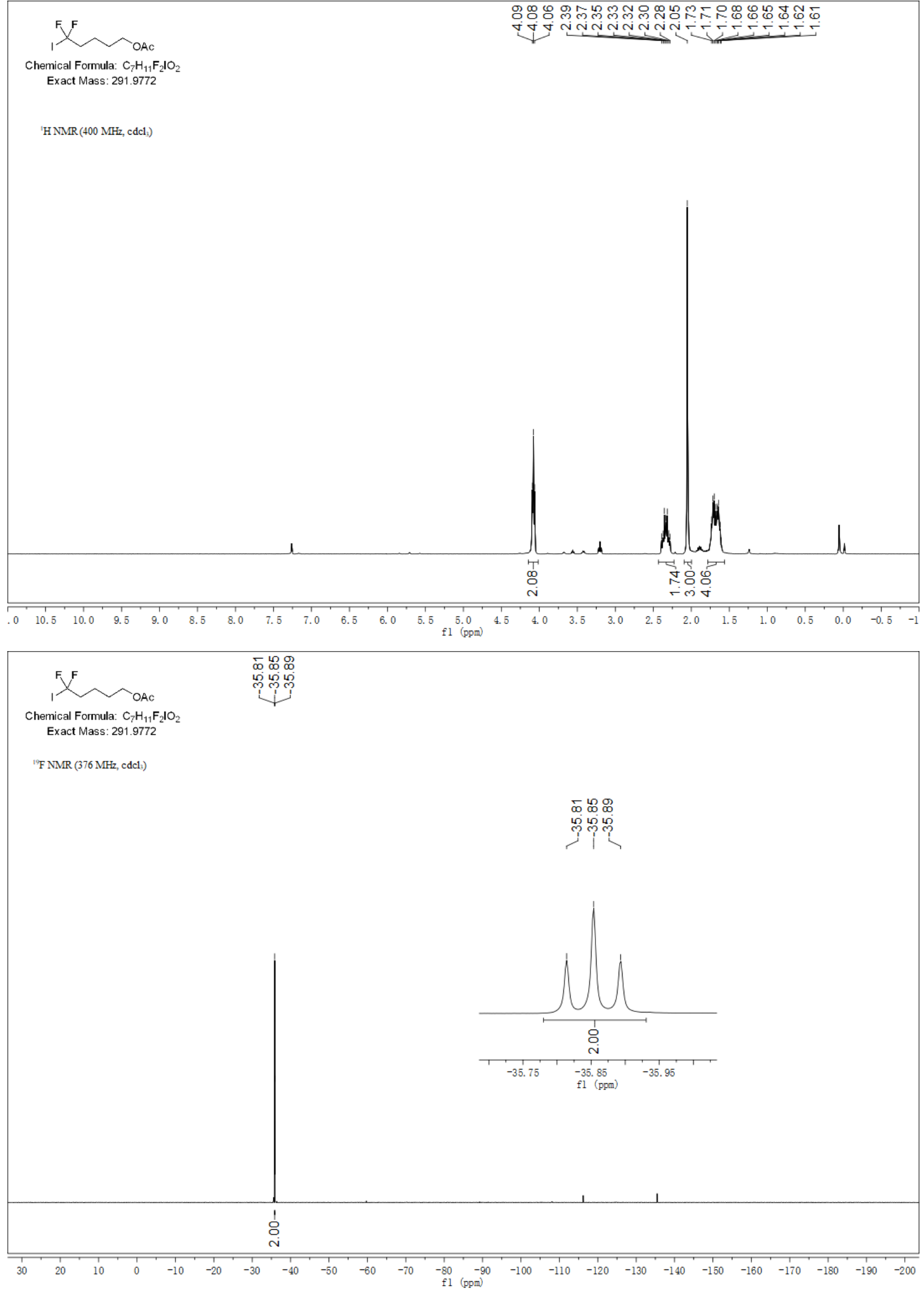


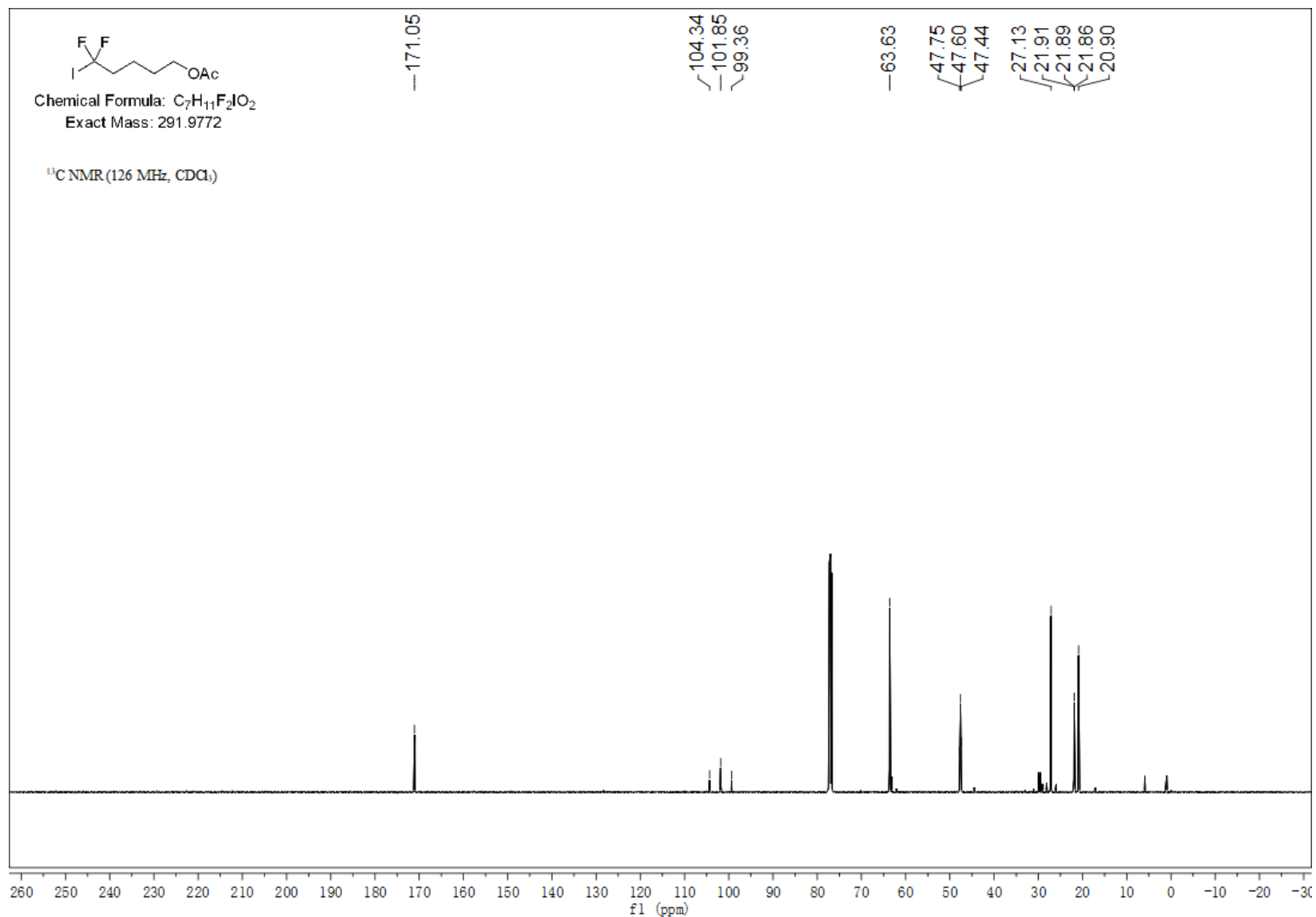

tert-Butyl(4,4-difluoro-4-iodobutoxy)dimethylsilane (2d).

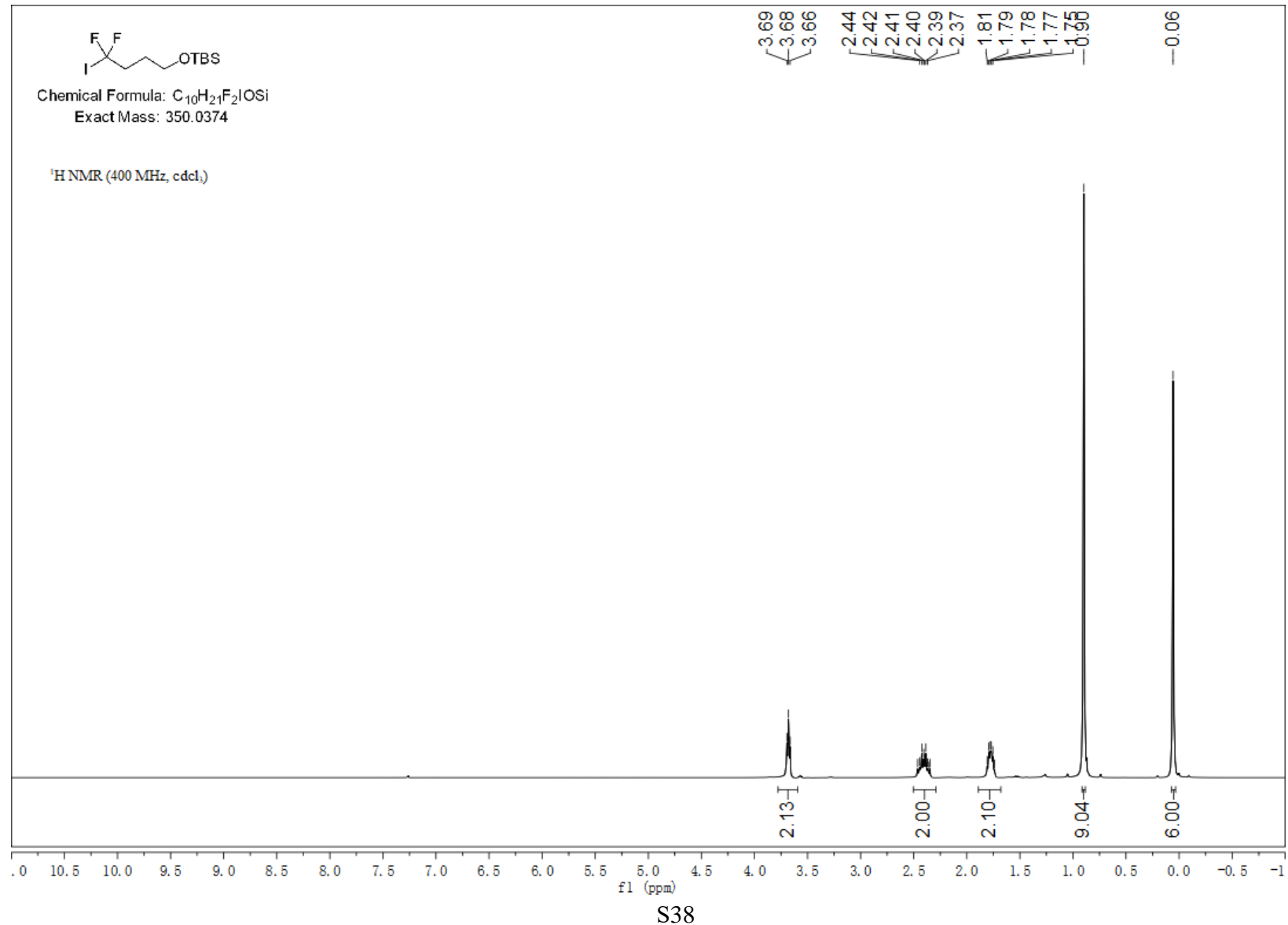



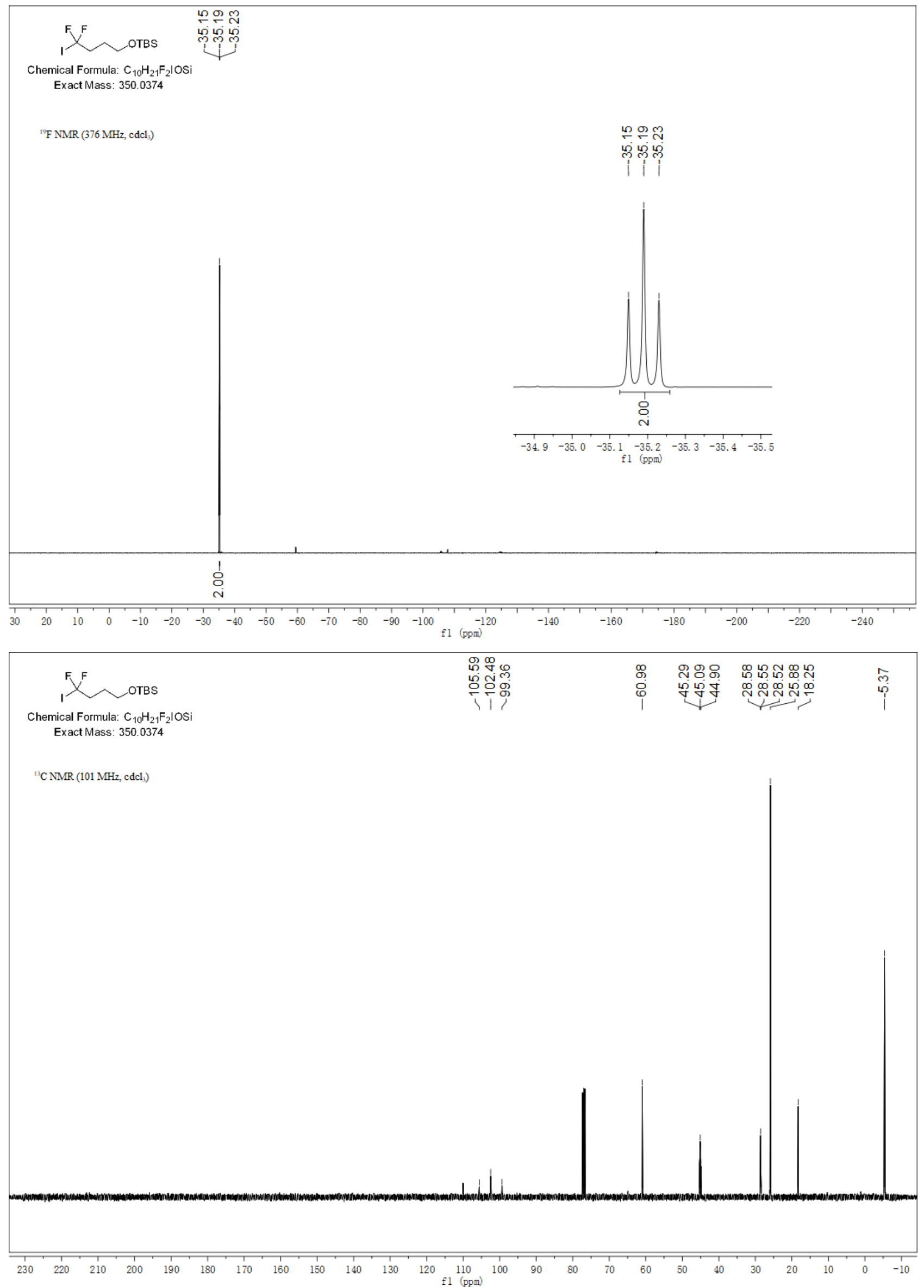
6-Chloro-1,1-difluoro-1-iodohexane (2e).
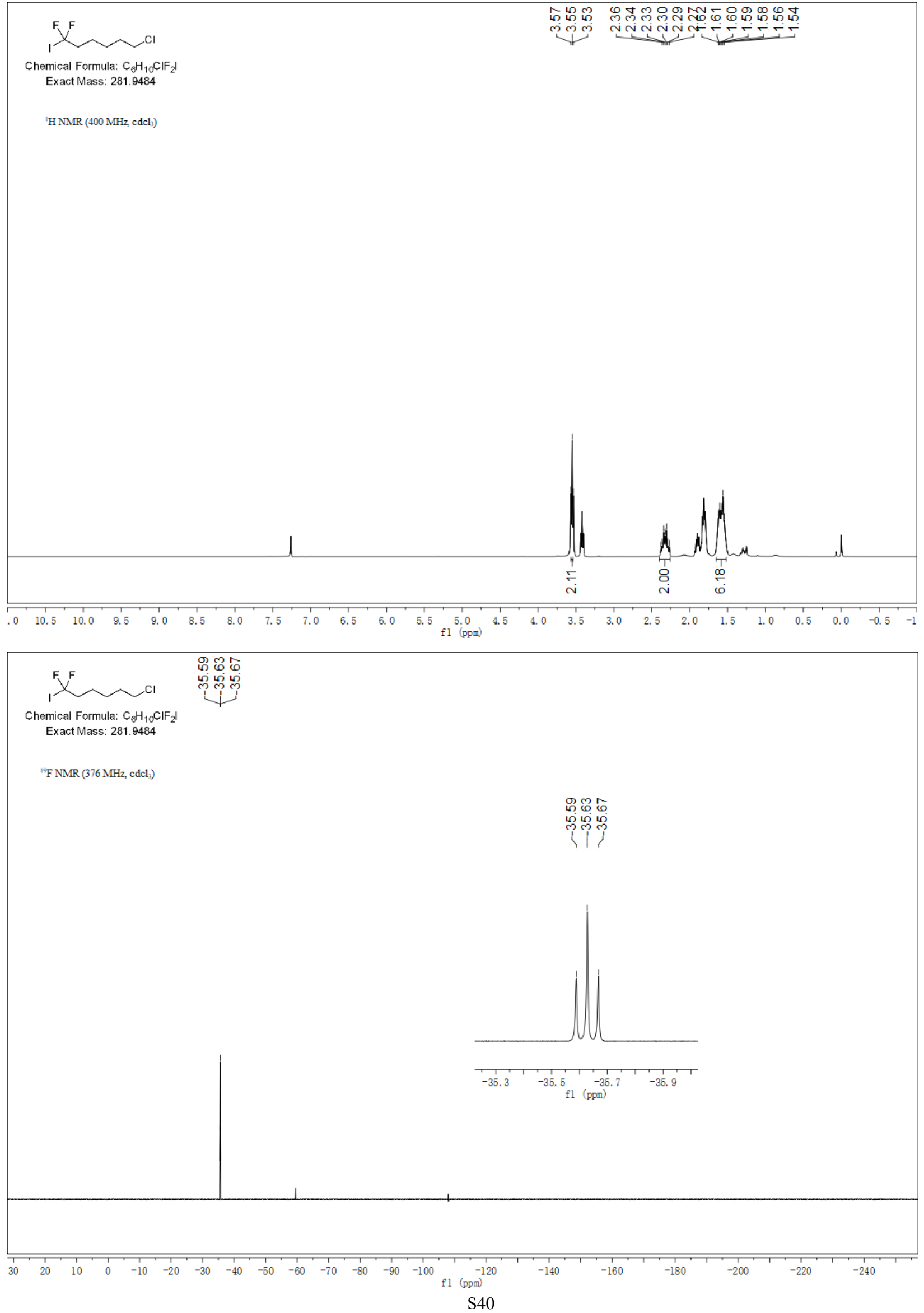


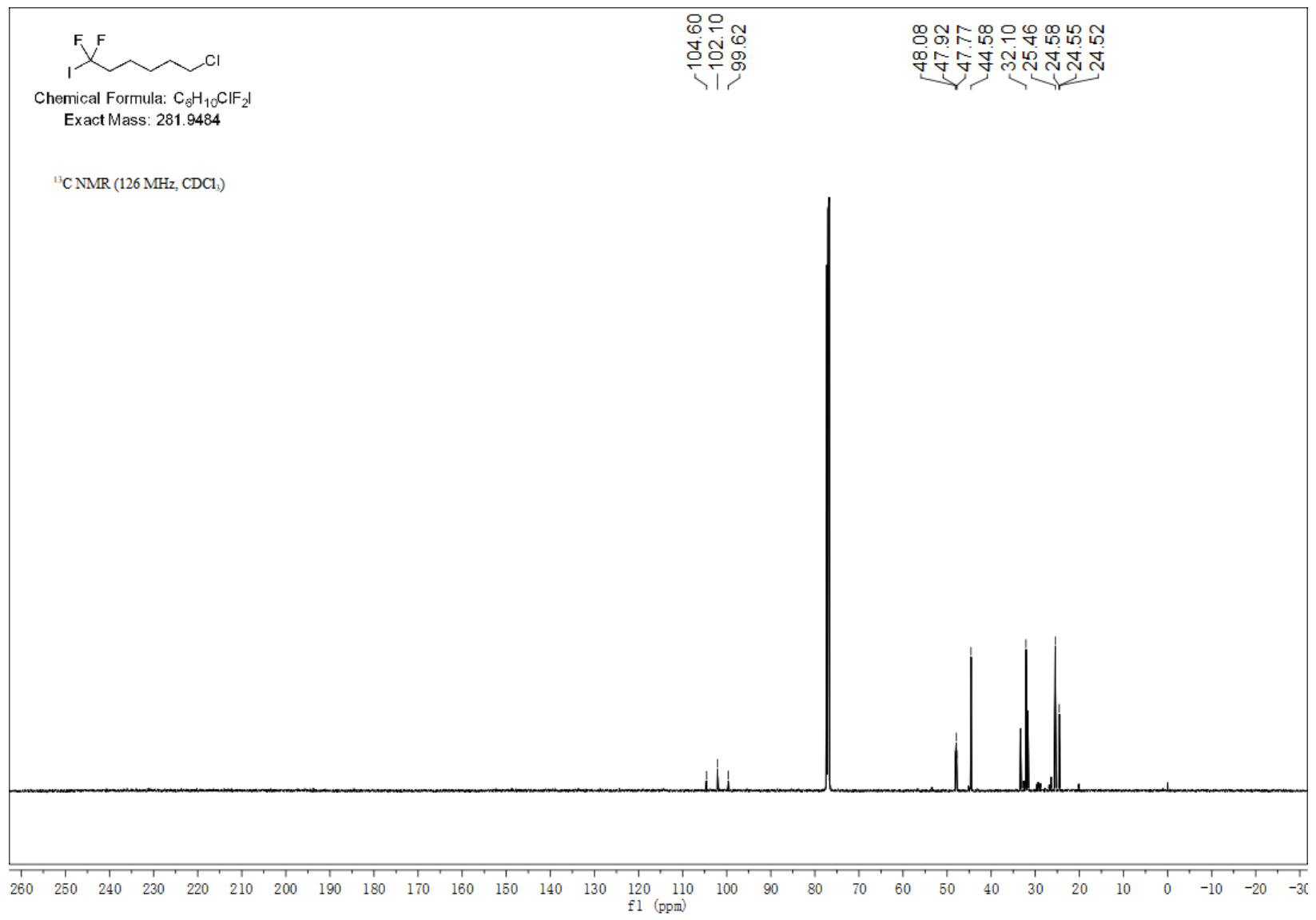

6,6-Difluoro-6-iodohexanenitrile (2f).

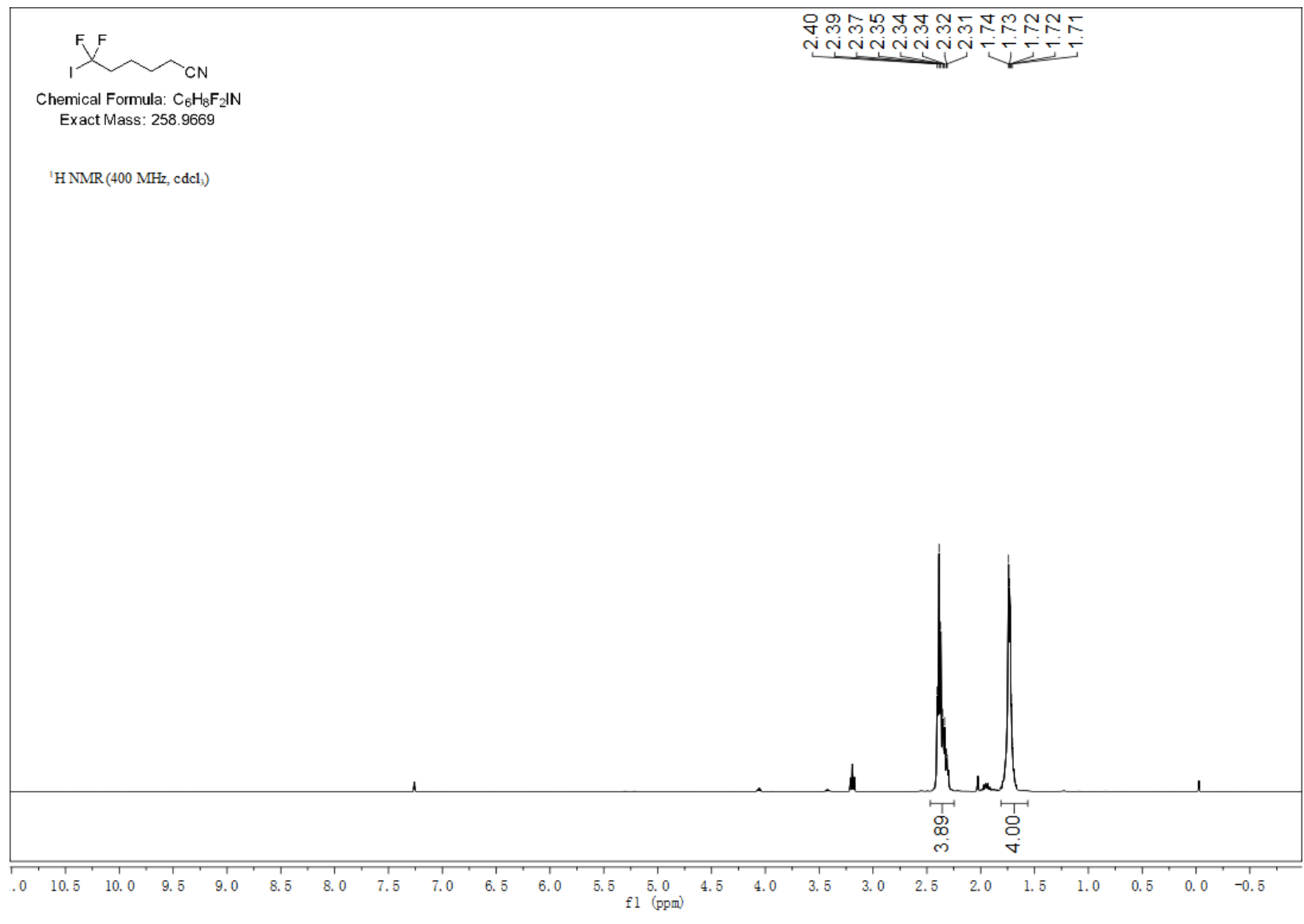




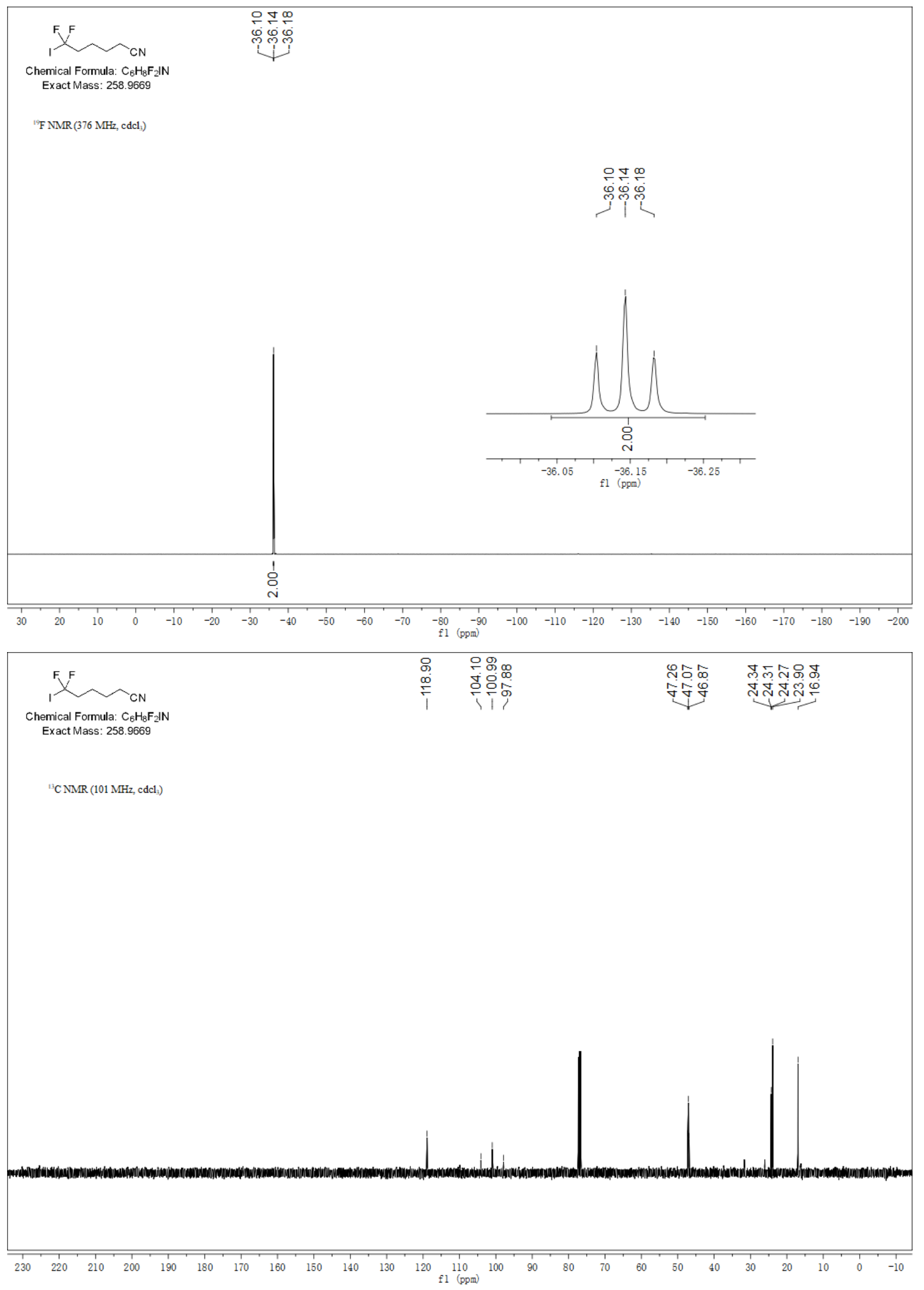


4,4-Difluoro-4-iodobutyl 4-chlorobenzoate $(2 \mathrm{~h})$.
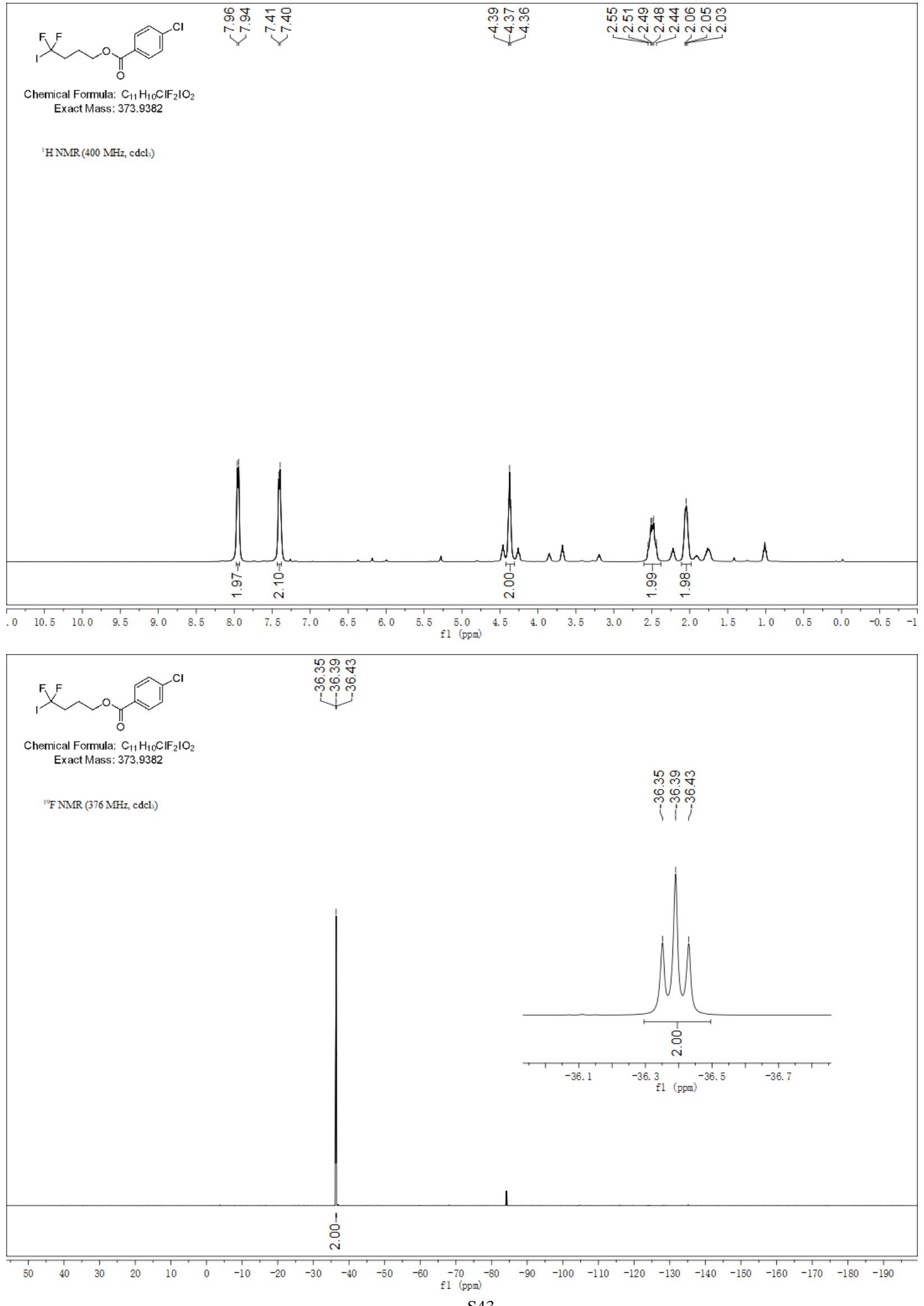


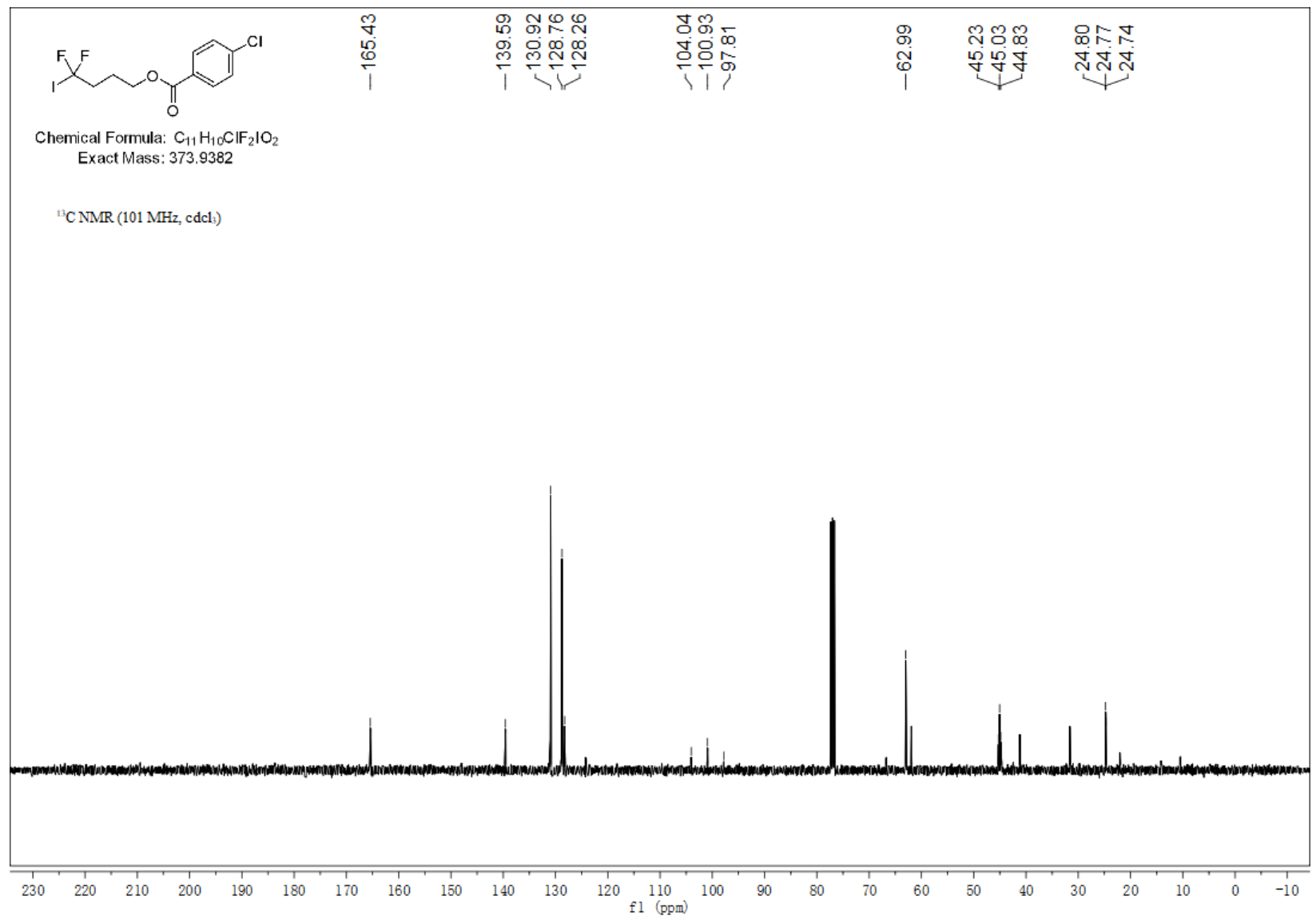

3,3-Difluoro-1,7-diphenylheptan-4-one (3a).

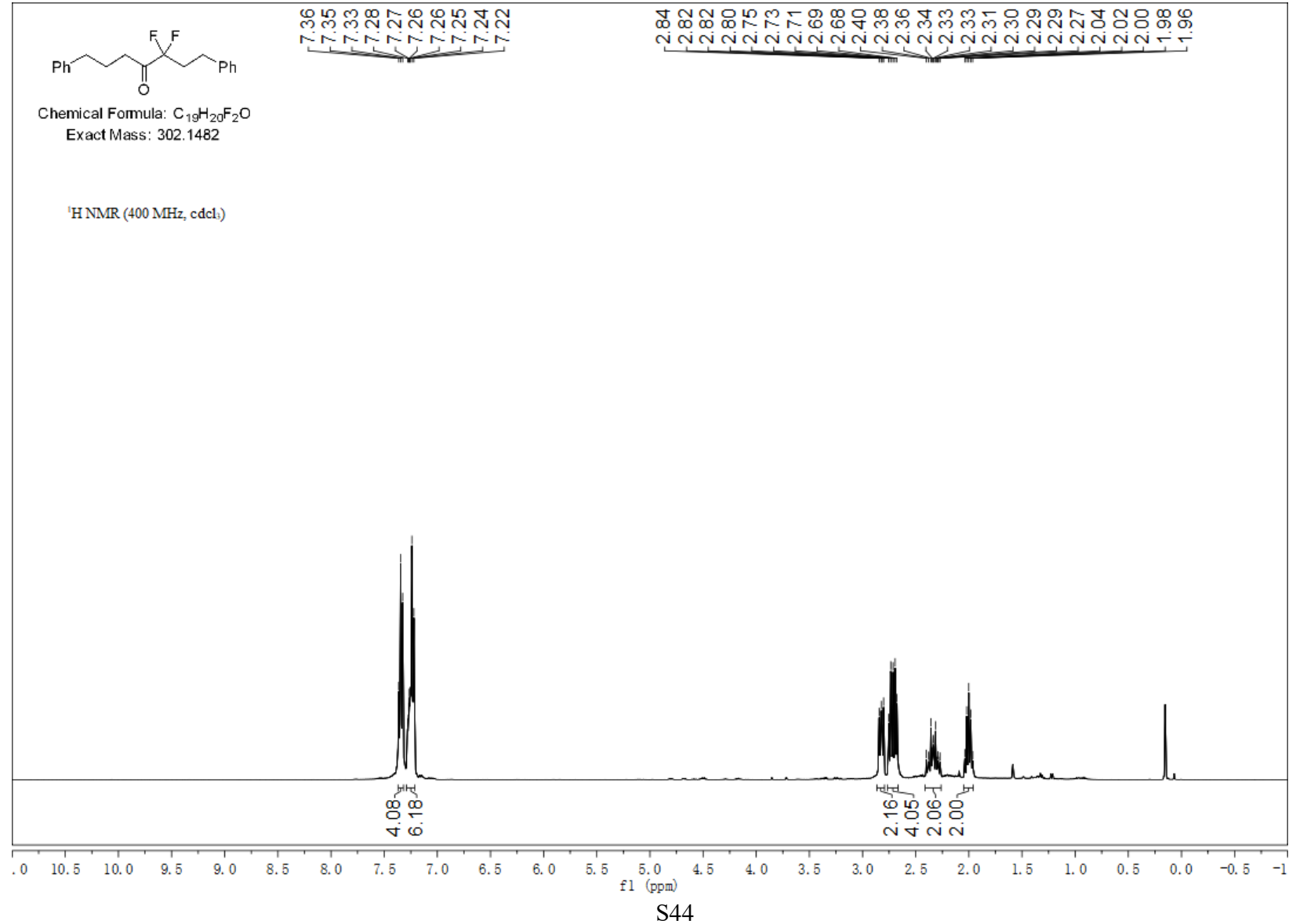




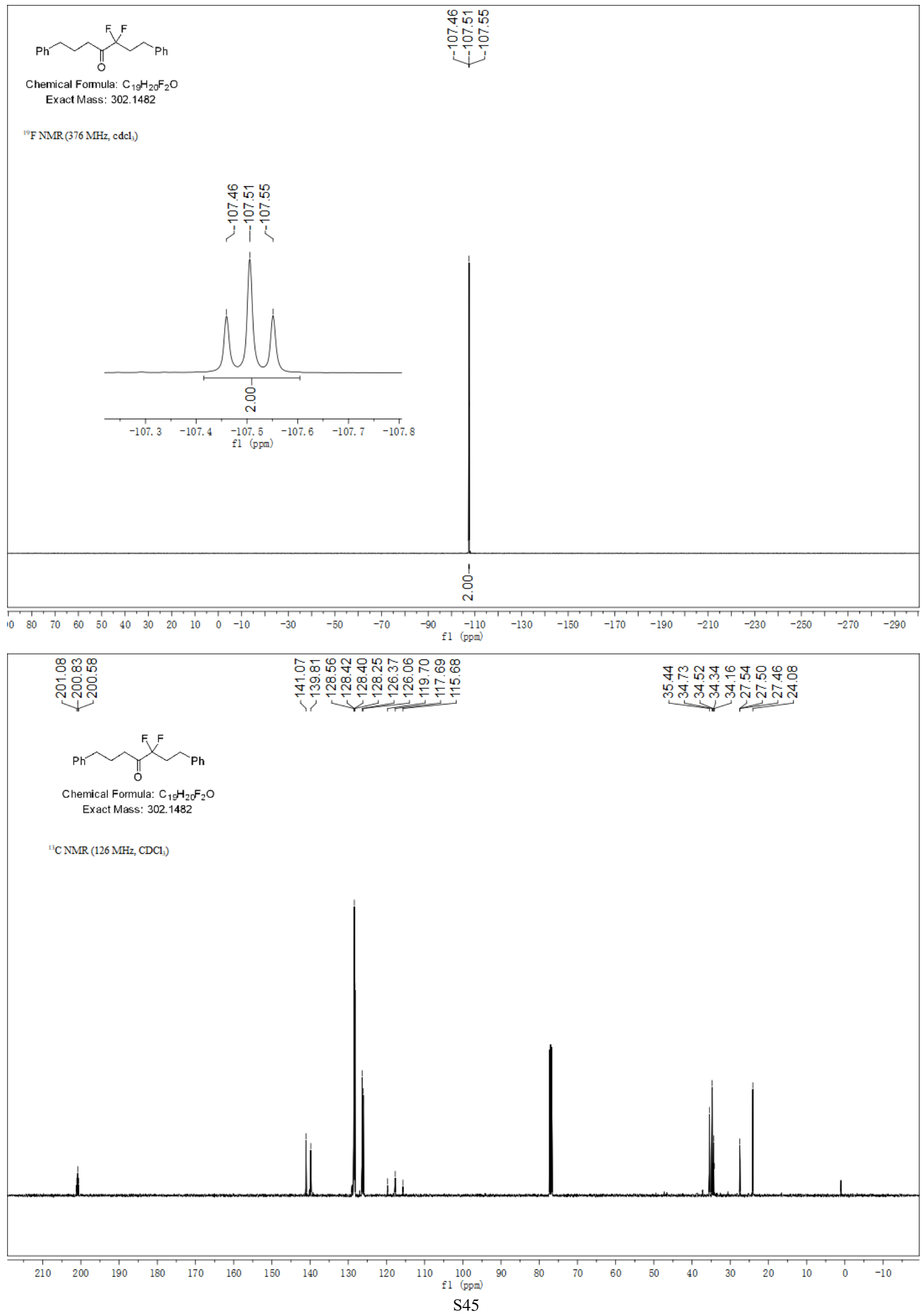


3,3-Difluoro-1,8-diphenyloctan-4-one (3b).
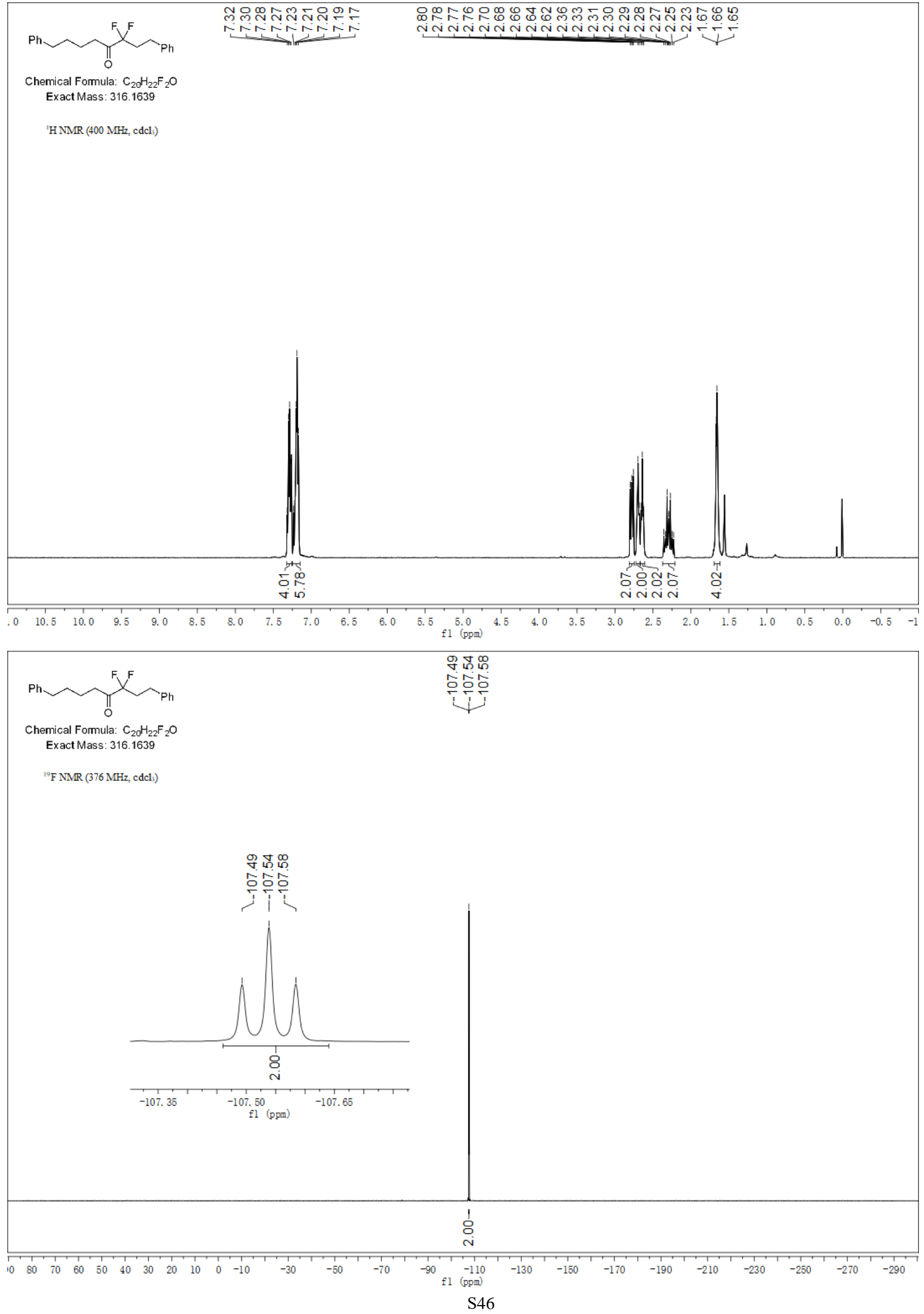


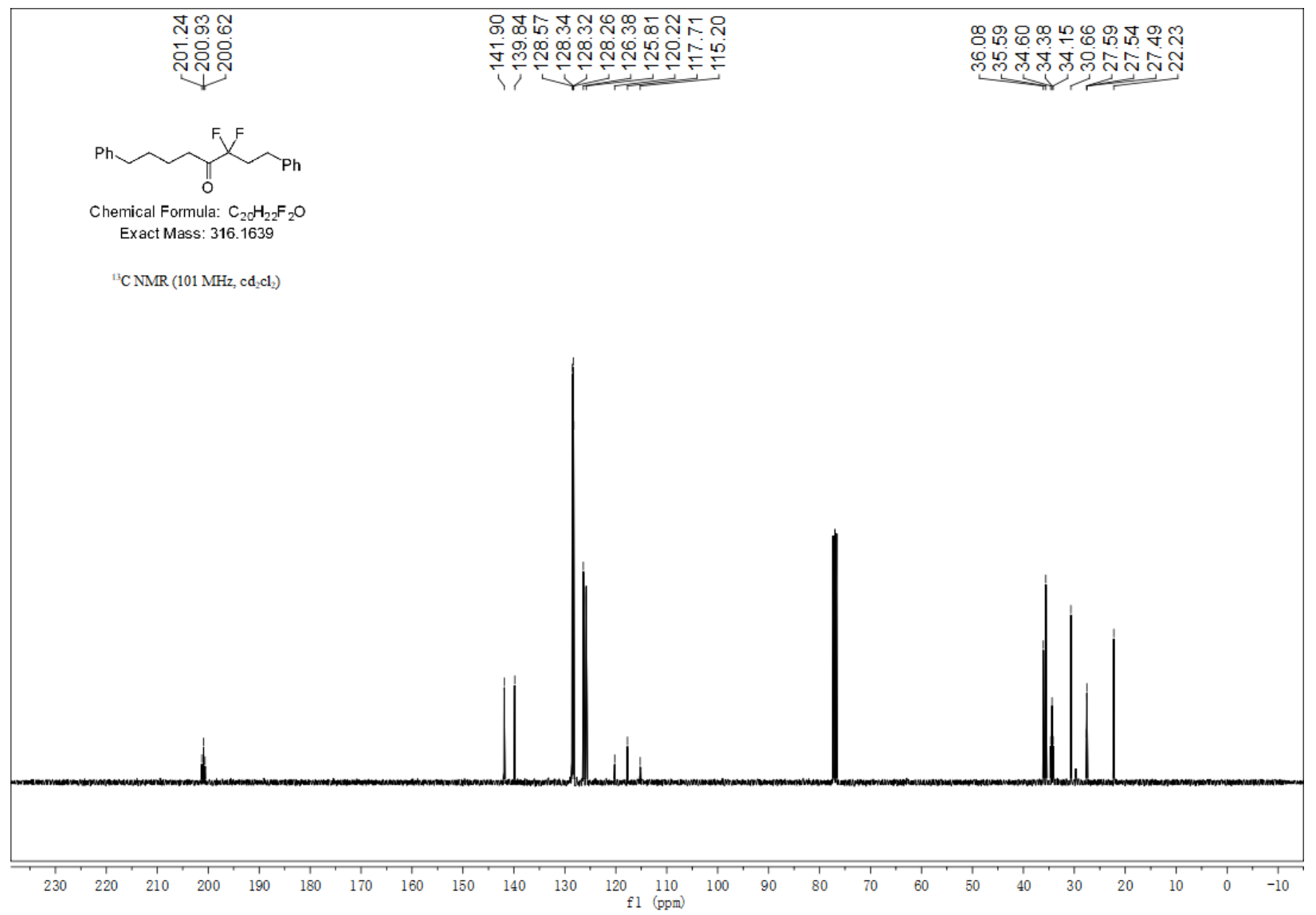

3,3-Difluoro-1-phenyldecan-4-one (3c).

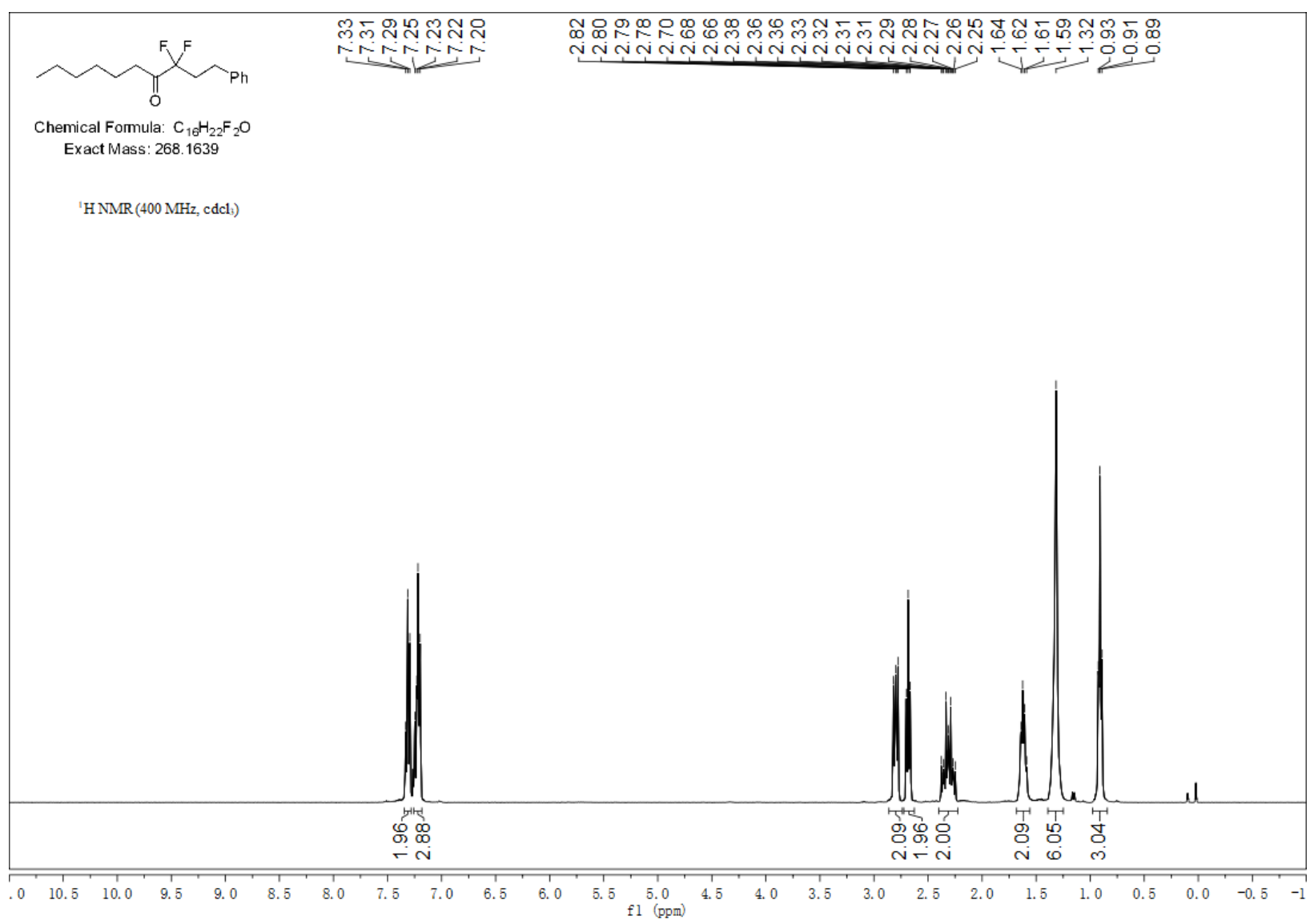



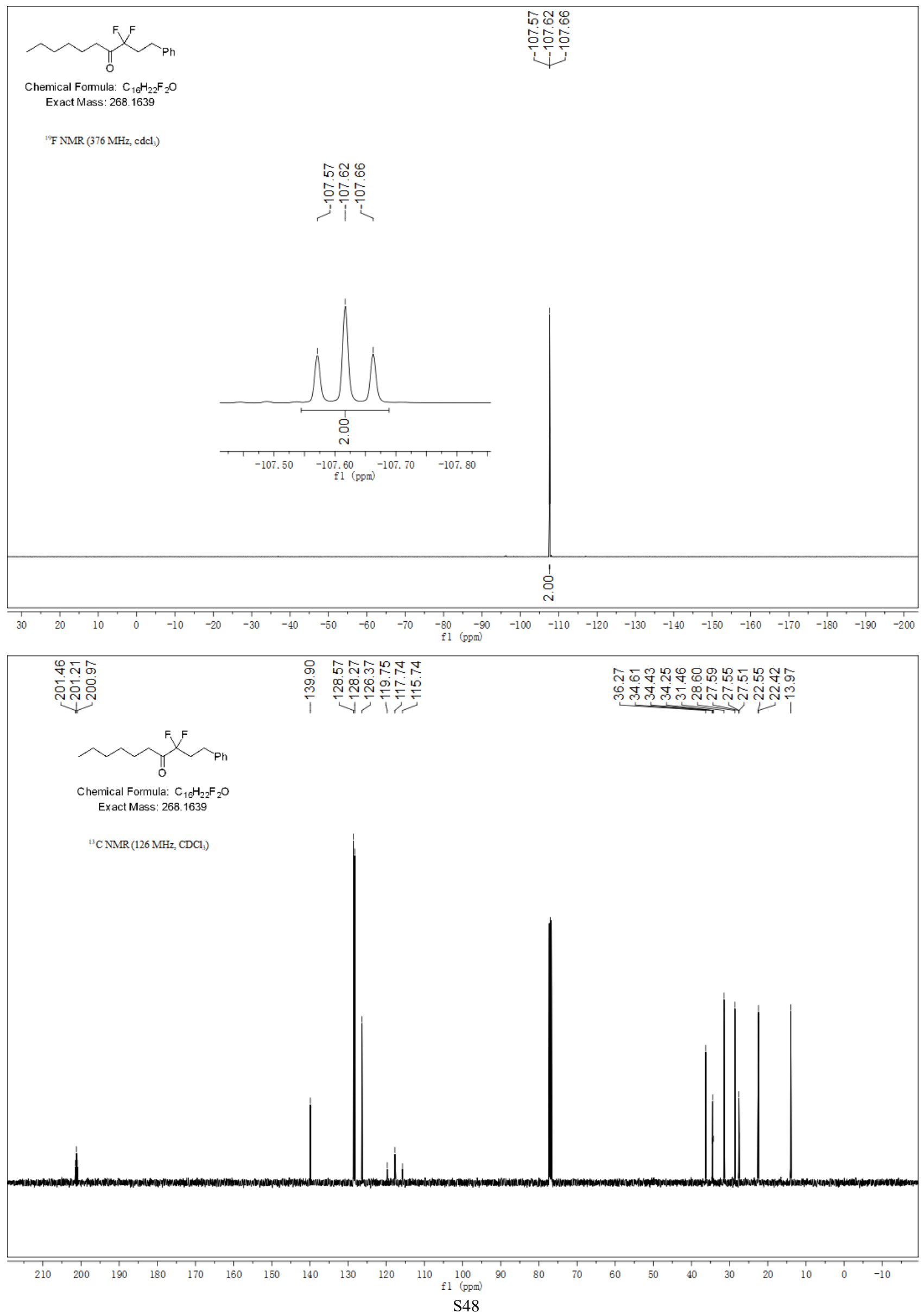
1-(4-Chlorophenyl)-4,4-difluoro-6-phenylhexan-3-one (3d).
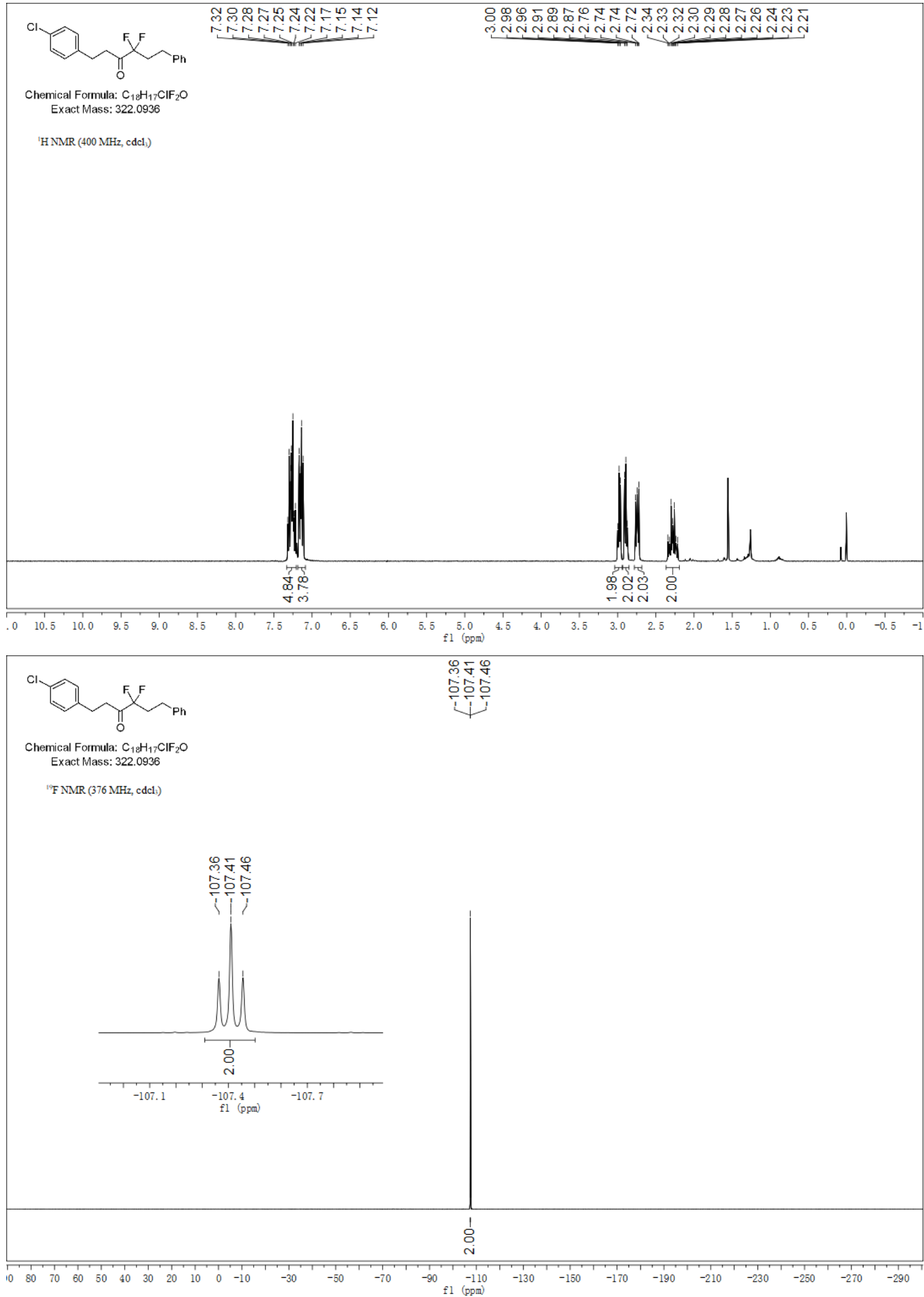


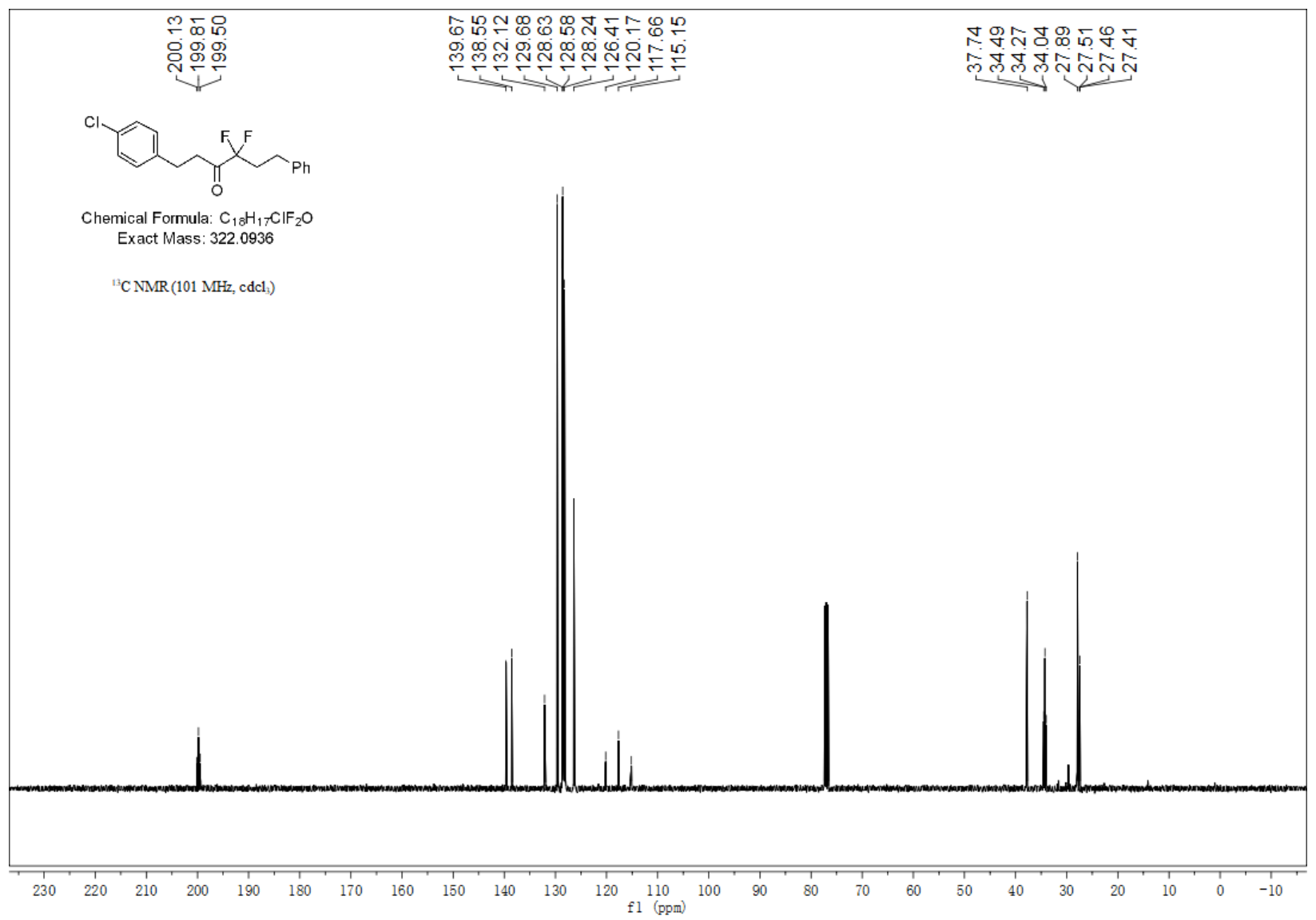

1-(4-(tert-Butyl)phenyl)-4,4-difluoro-6-phenylhexan-3-one (3e).

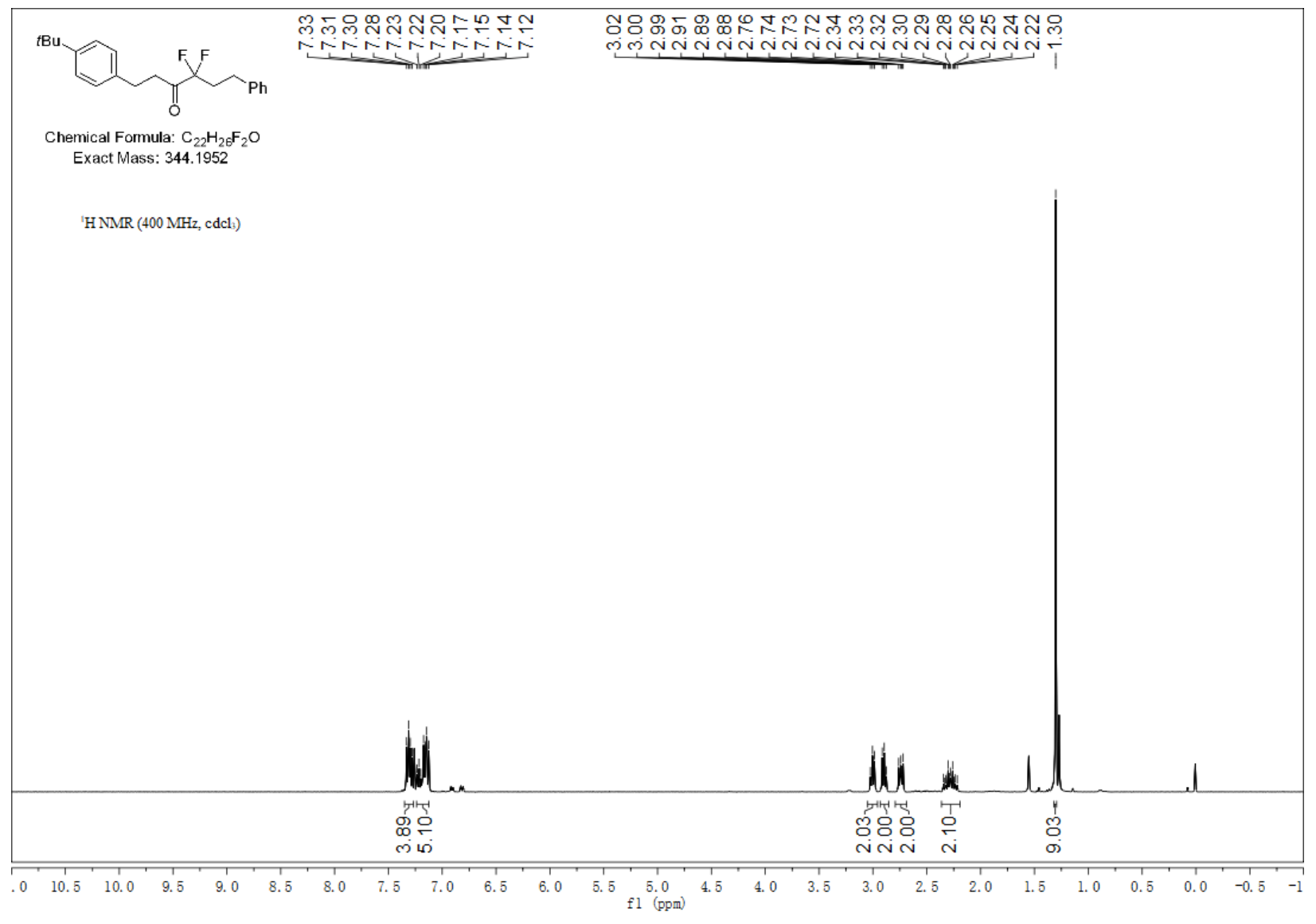



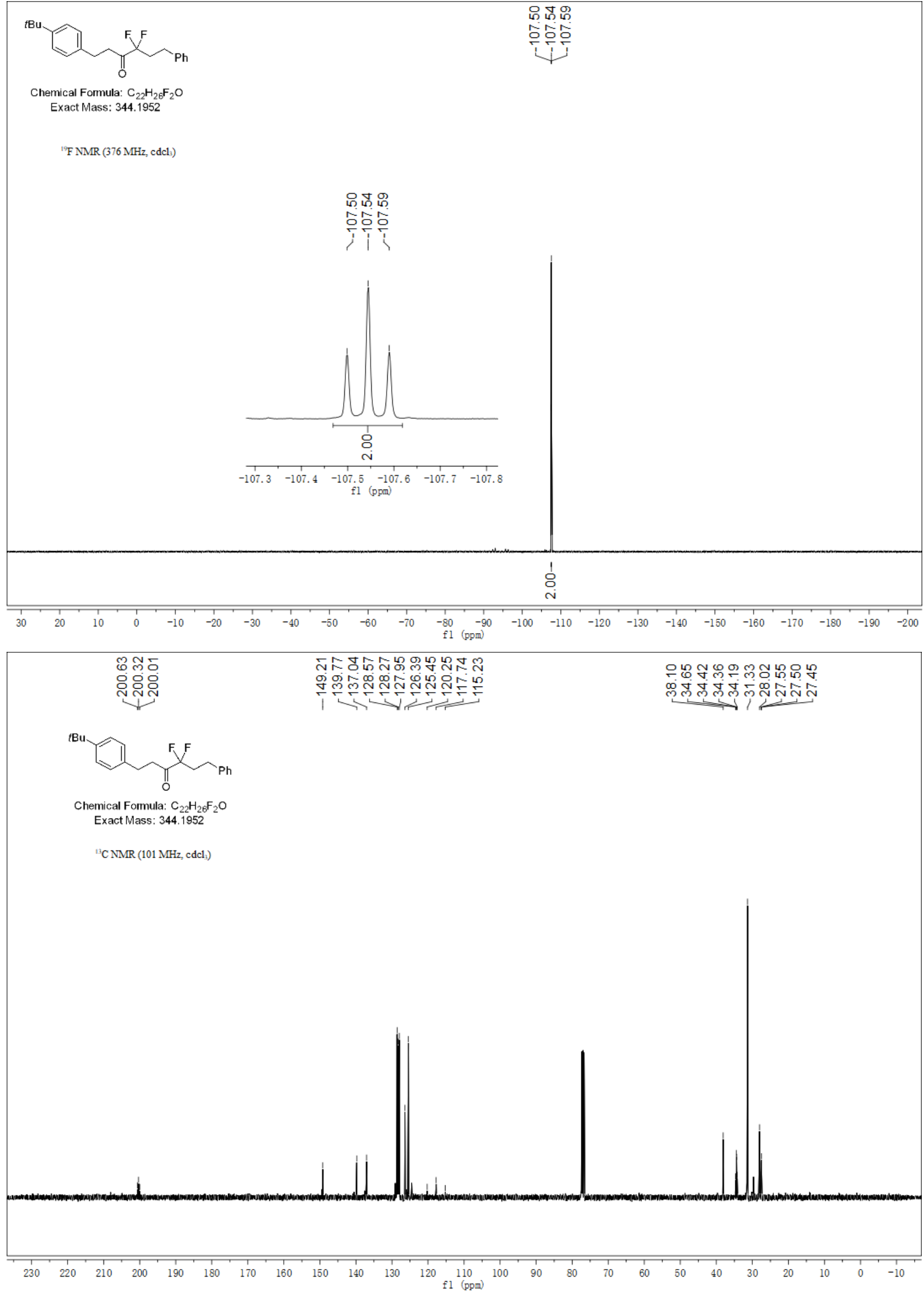
7-((tert-Butyldimethylsilyl)oxy)-3,3-difluoro-1-phenylheptan-4-one (3f).
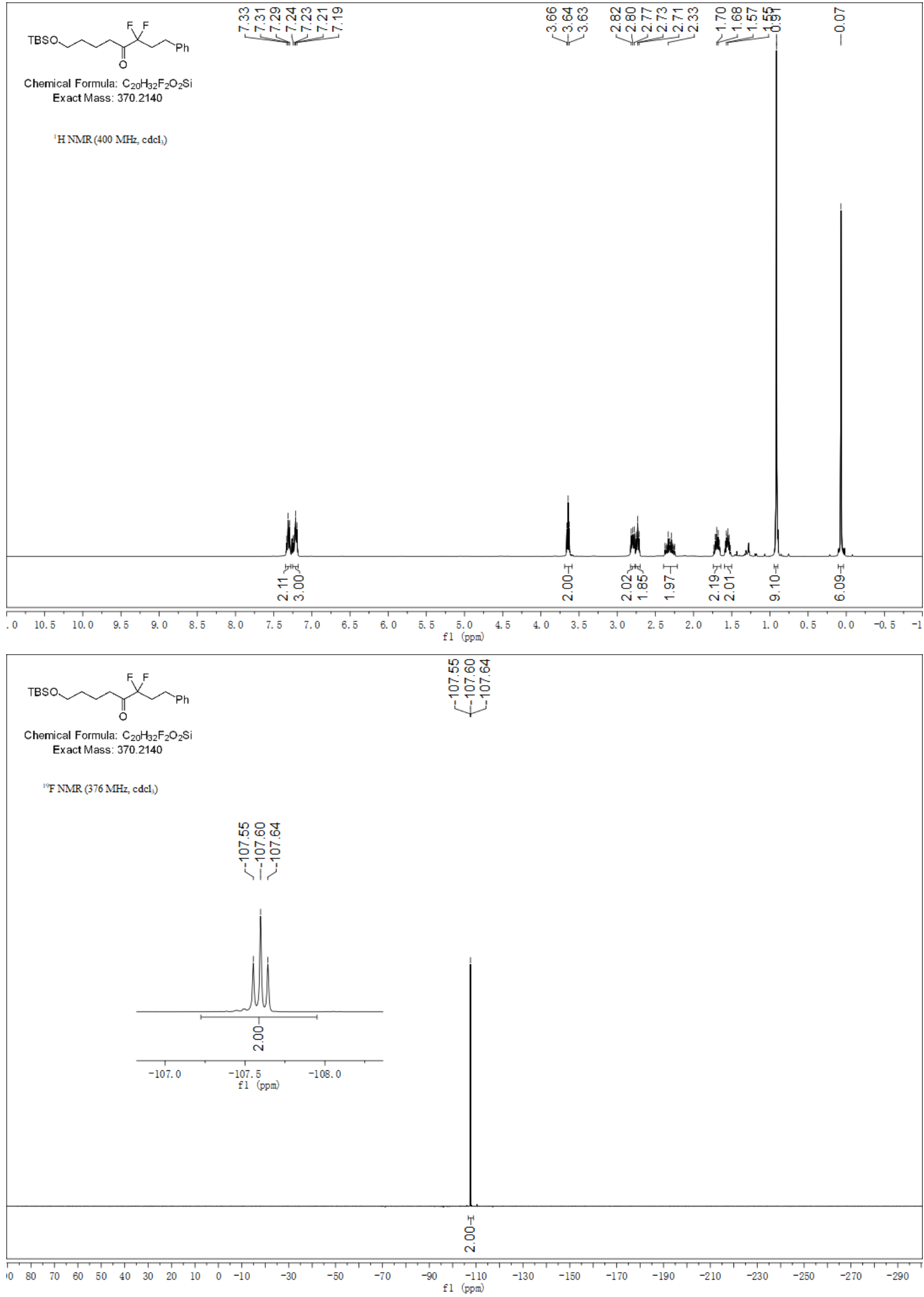


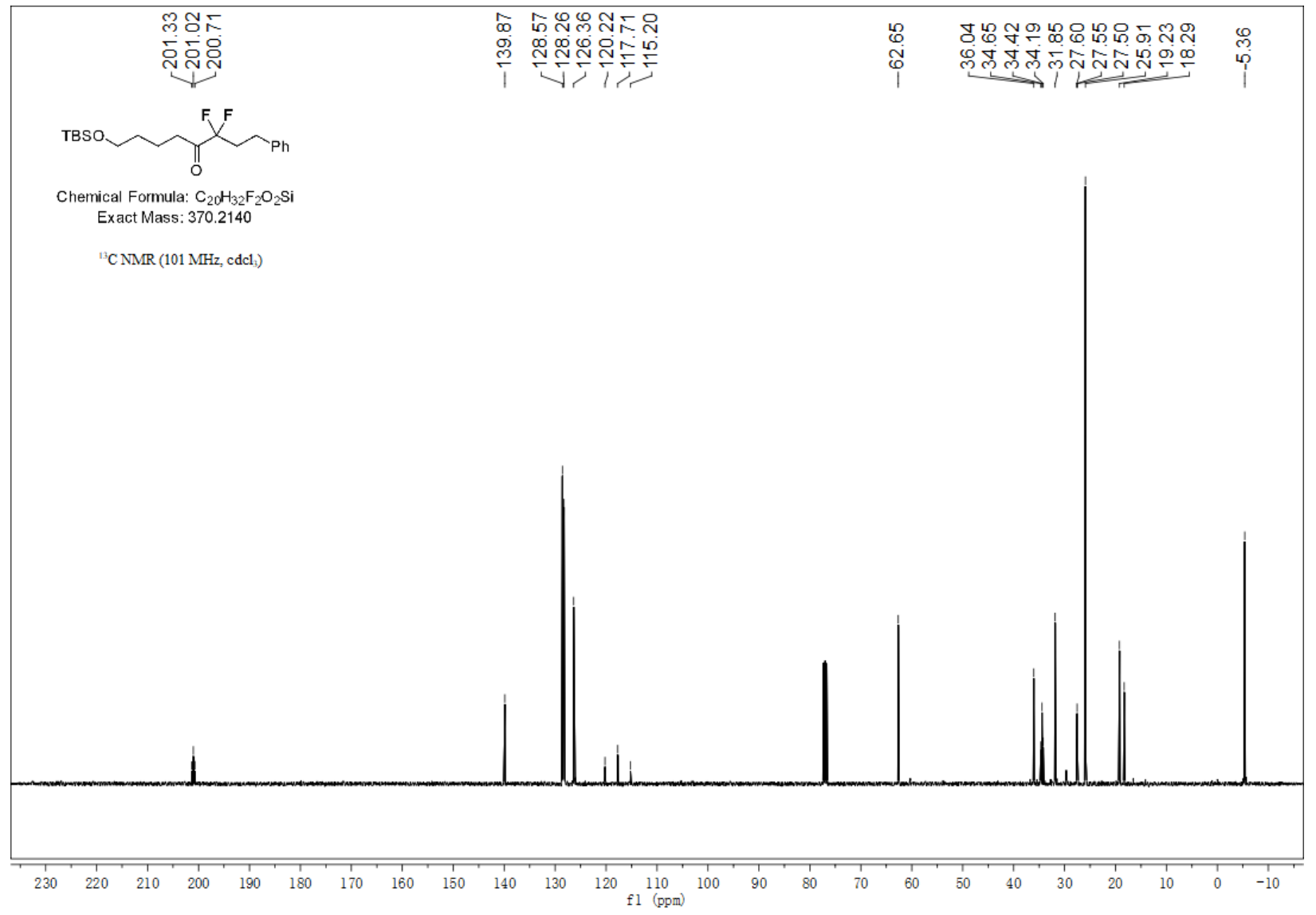

7-(Dimethyl(phenyl)silyl)-3,3-difluoro-1-phenylheptan-4-one (3g).

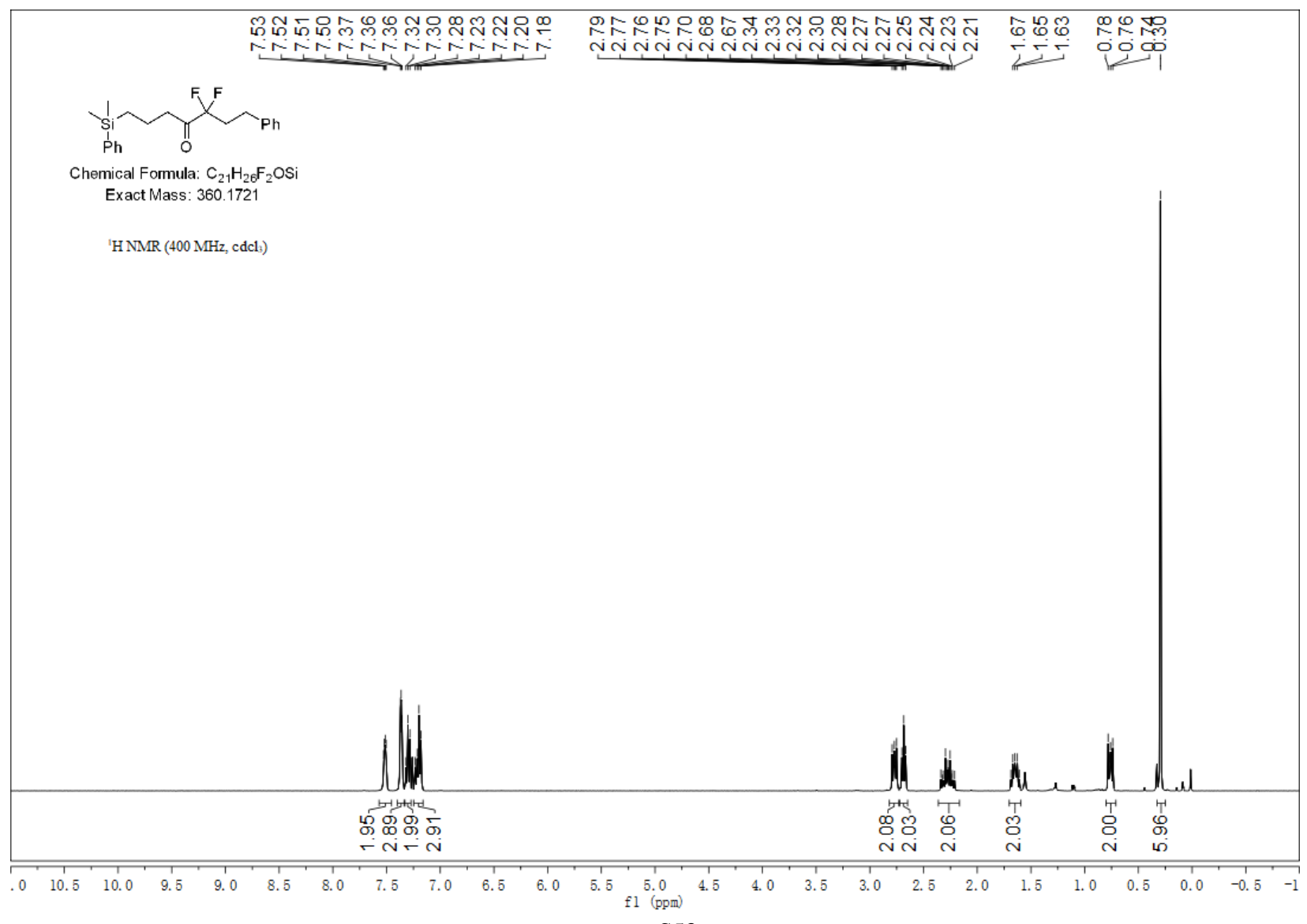



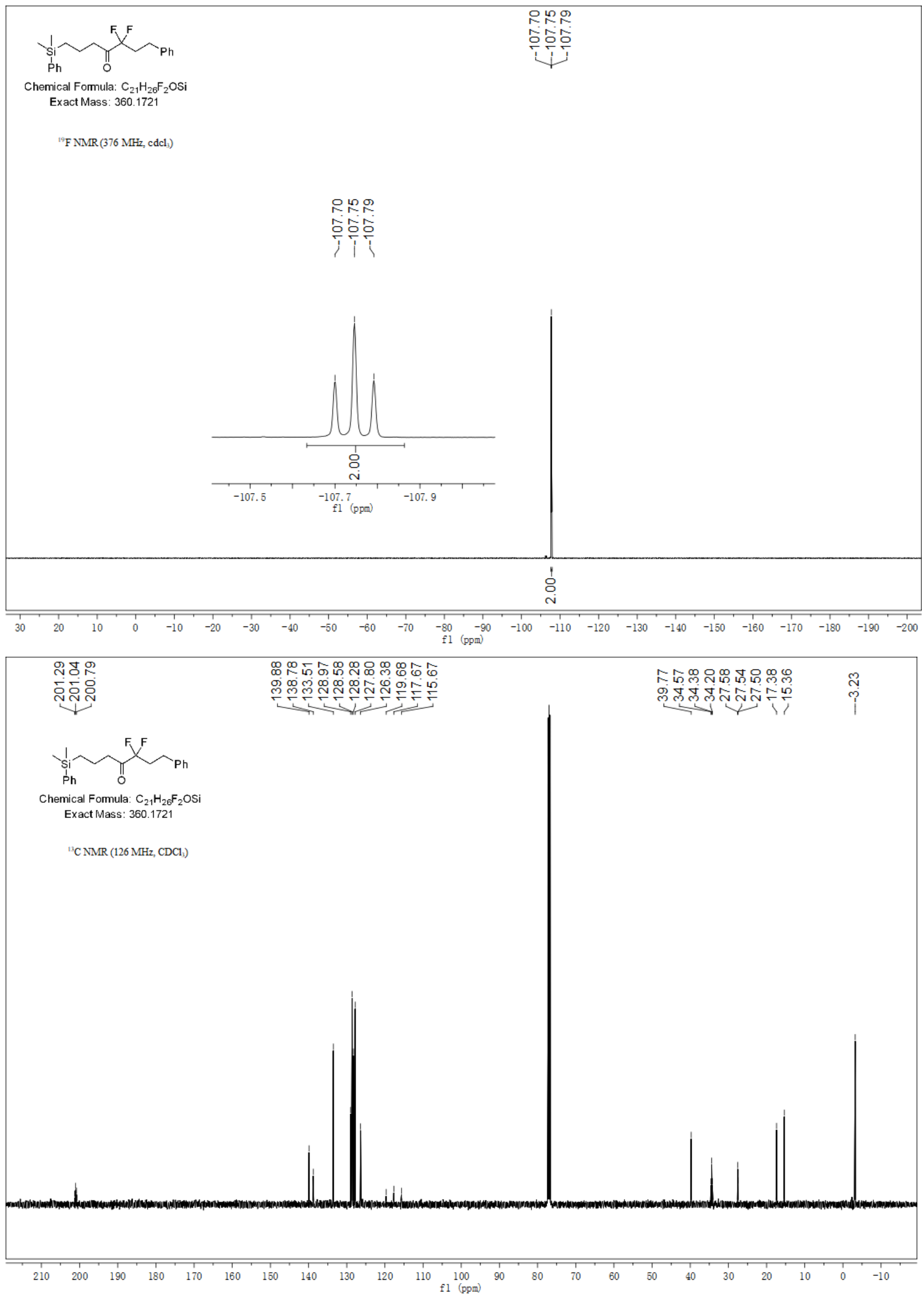
tert-Butyl 6,6-difluoro-5-0xo-8-phenyloctanoate (3h).
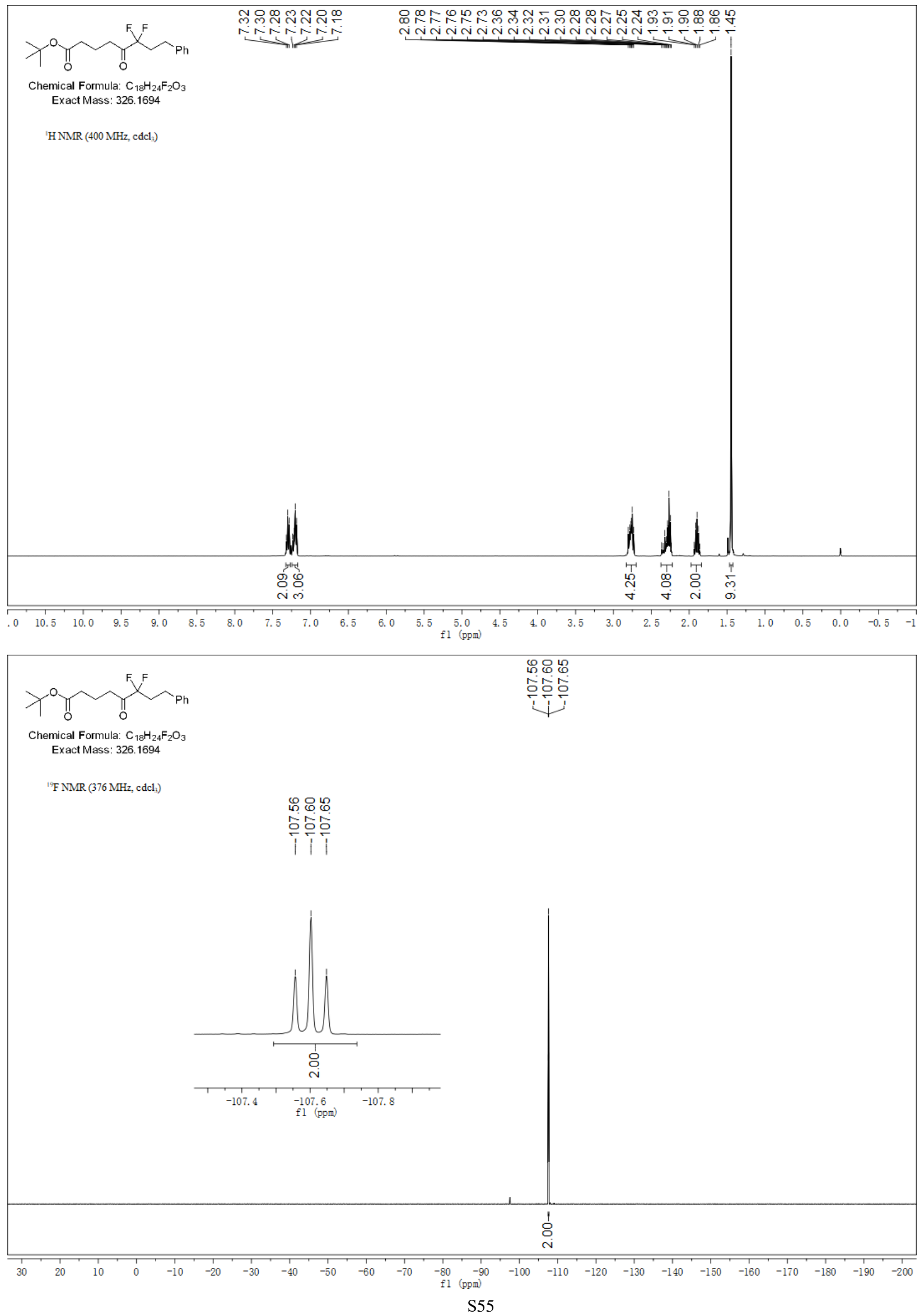


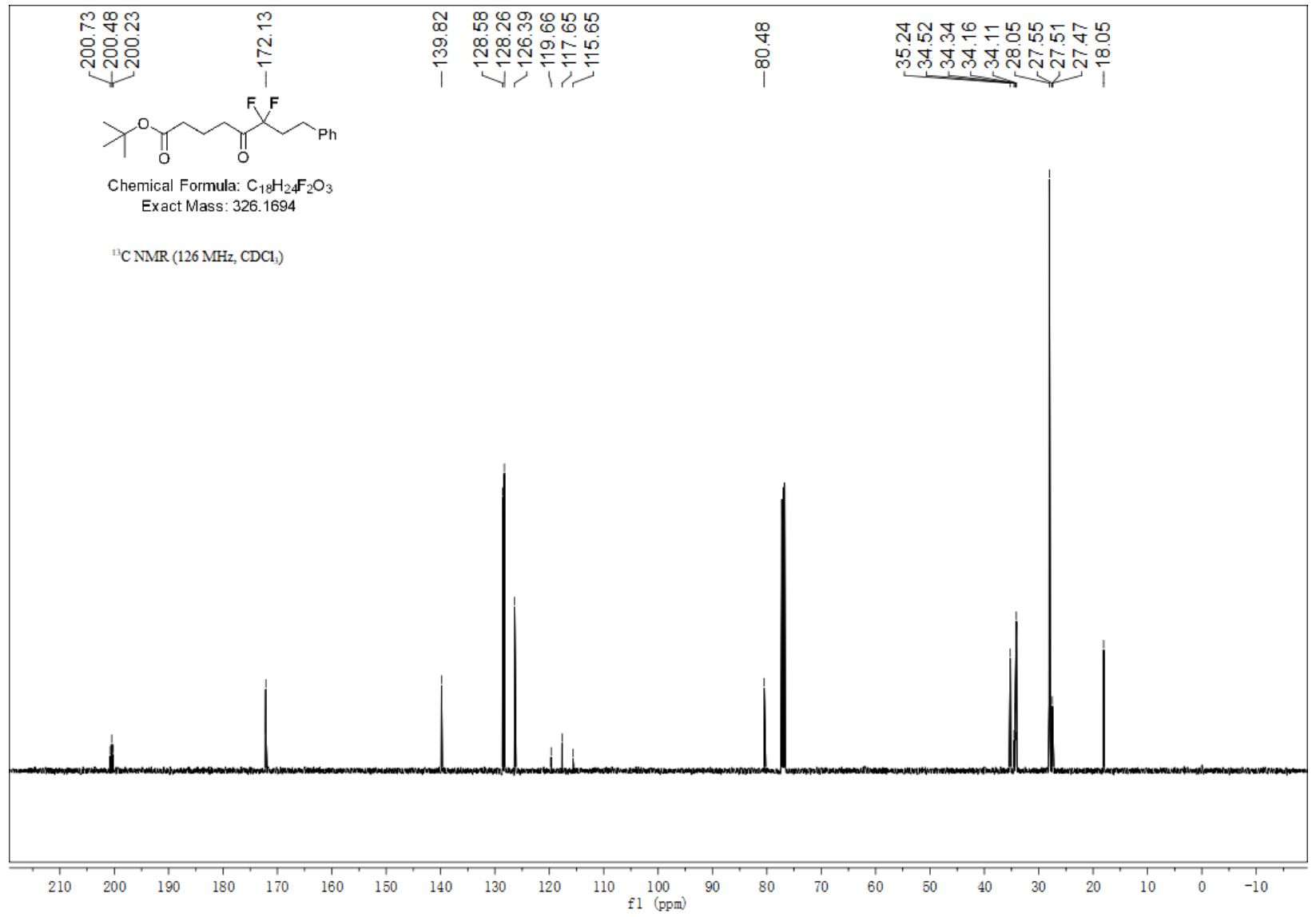

3,3-Difluoro-7-methoxy-1-phenylheptan-4-one (3i).

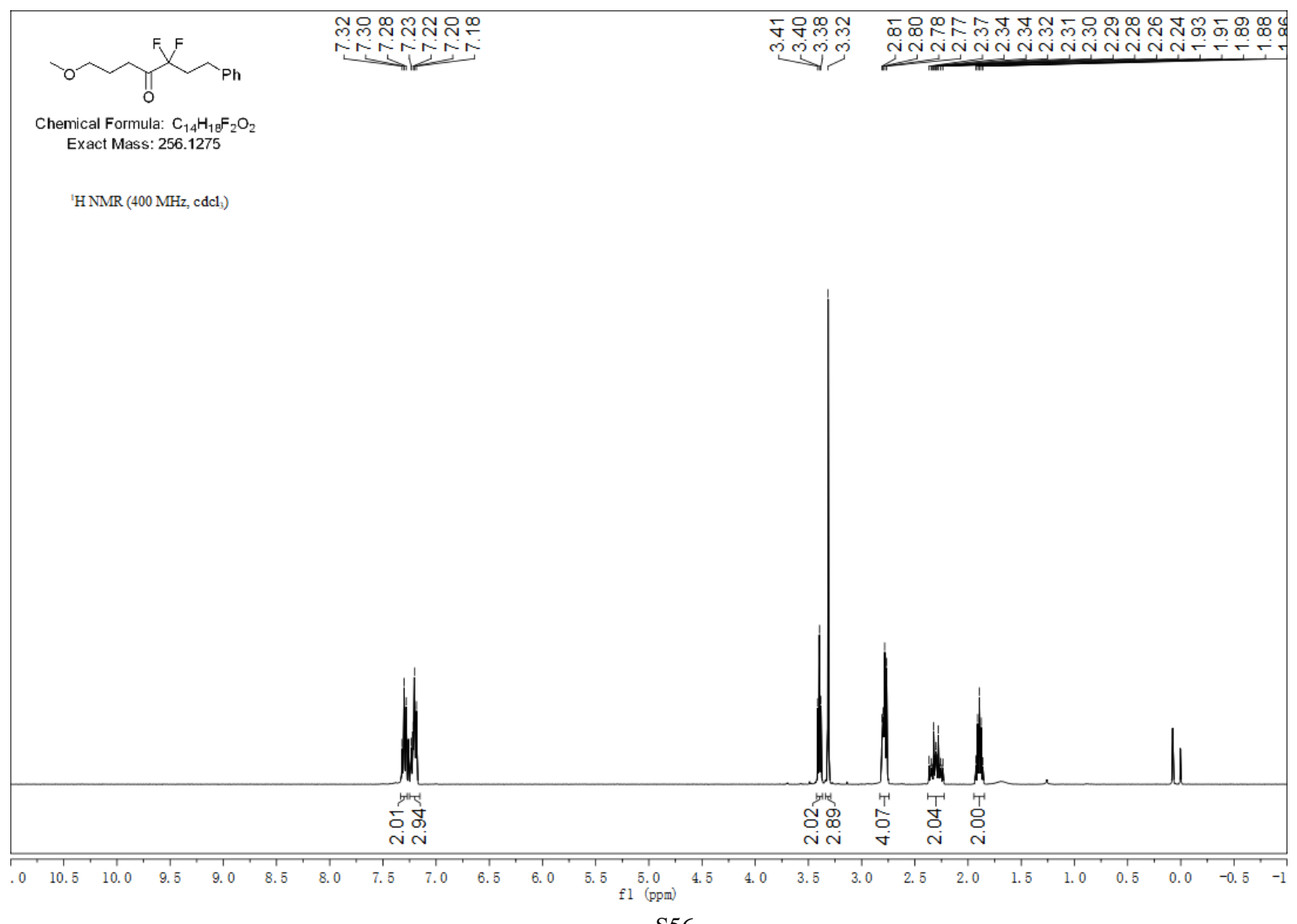



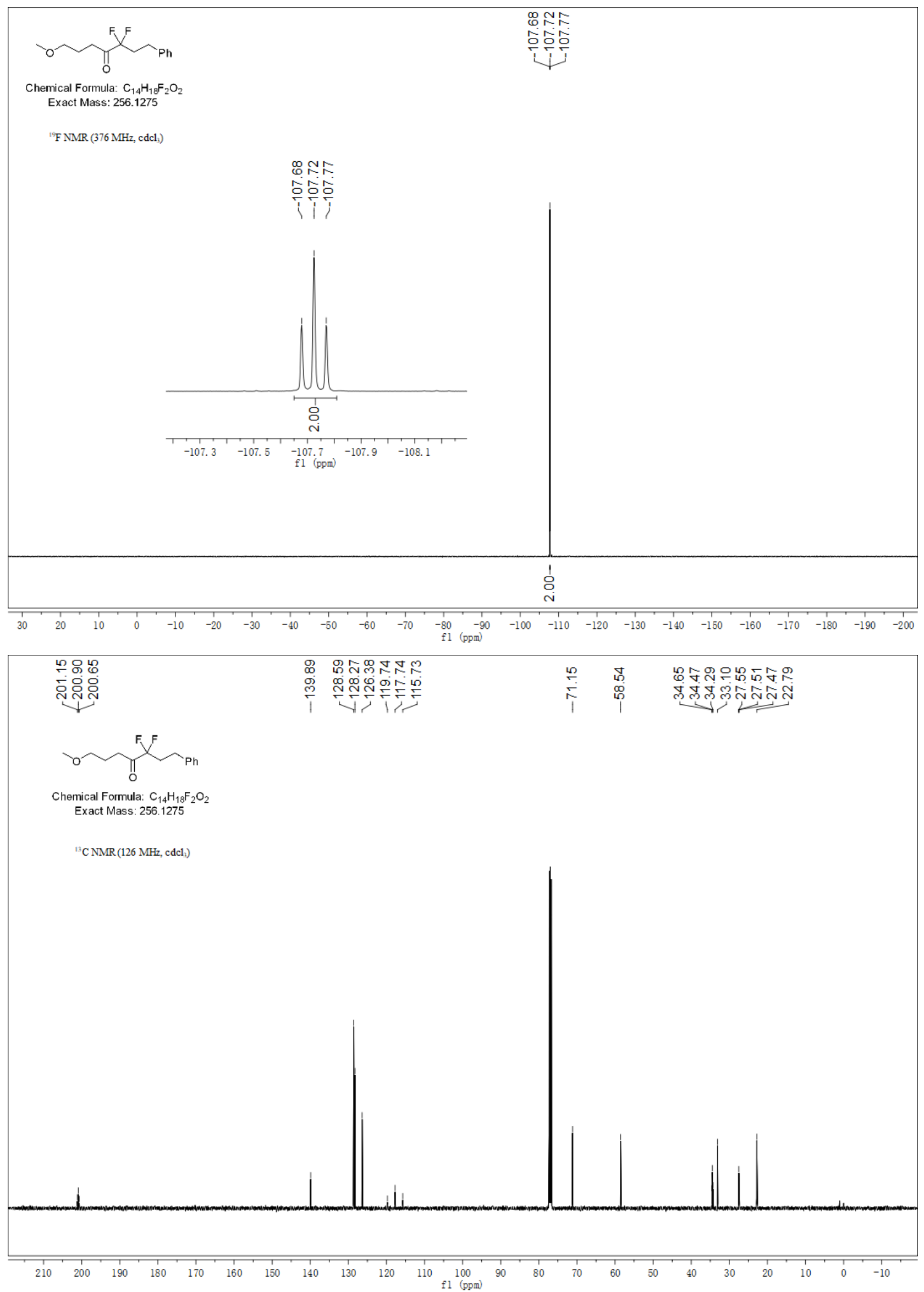
3,3-Difluoro-7-phenoxy-1-phenylheptan-4-one (3j).
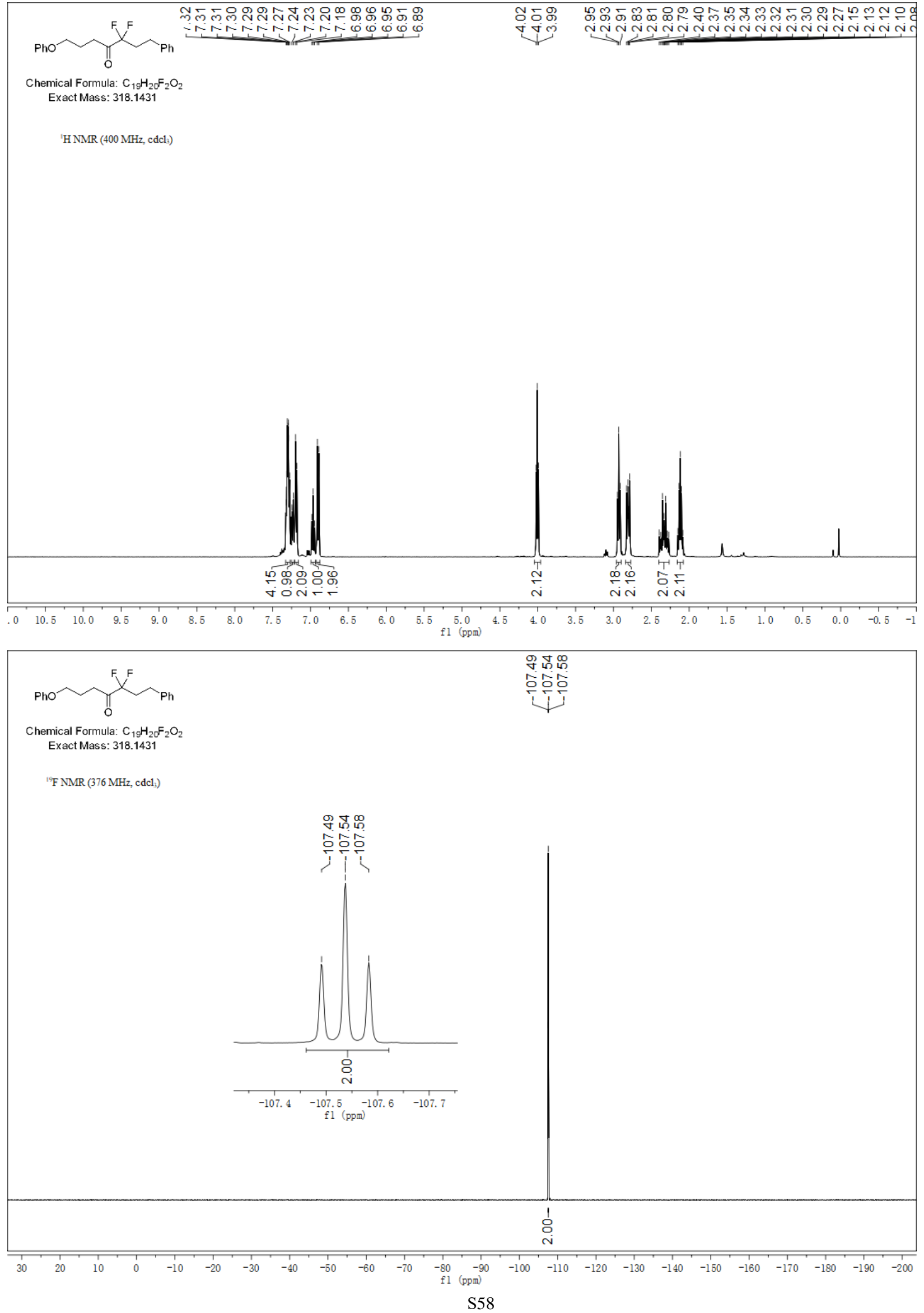


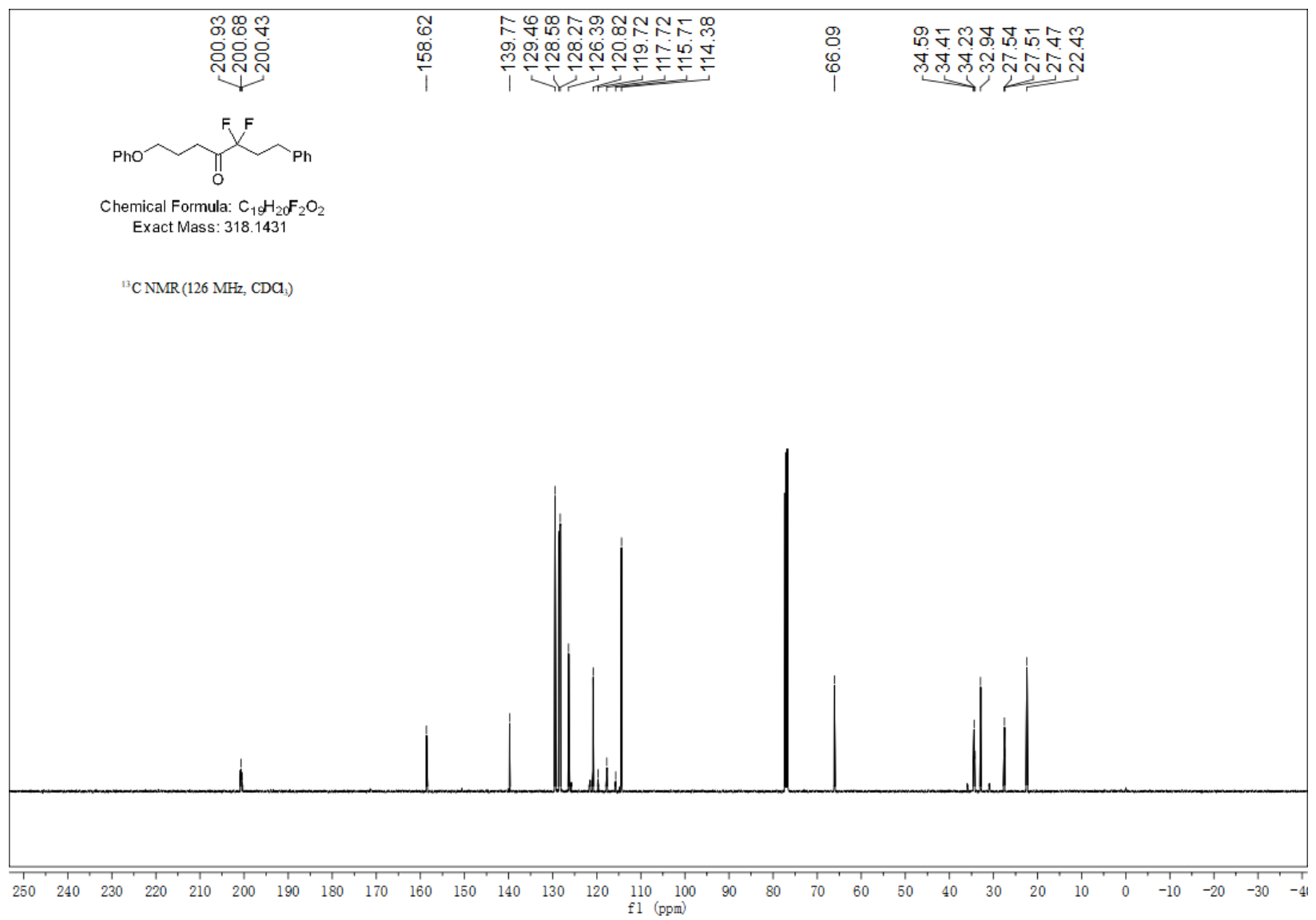

5,5-Difluoro-1-phenyltridecan-4-one (3k).

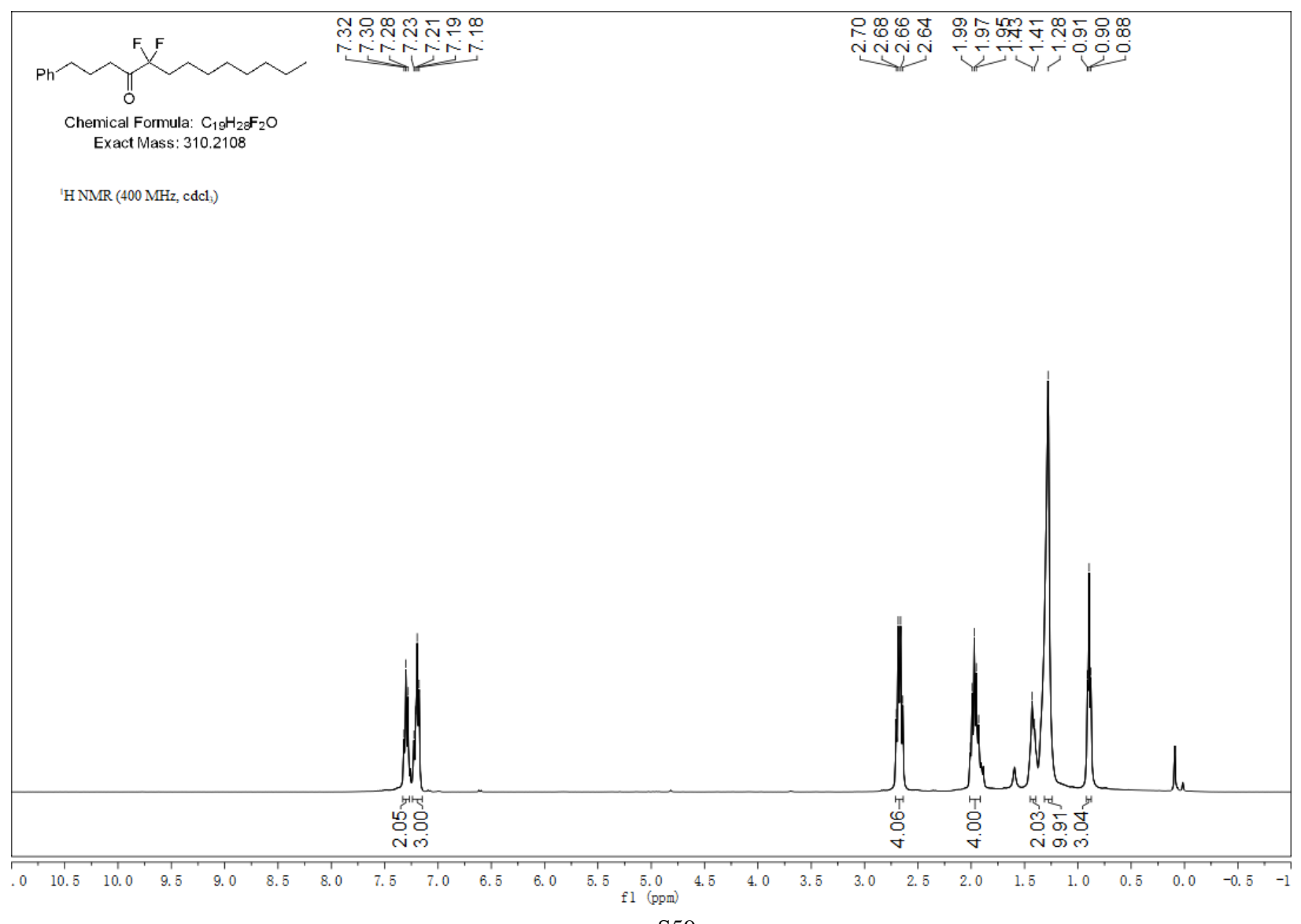



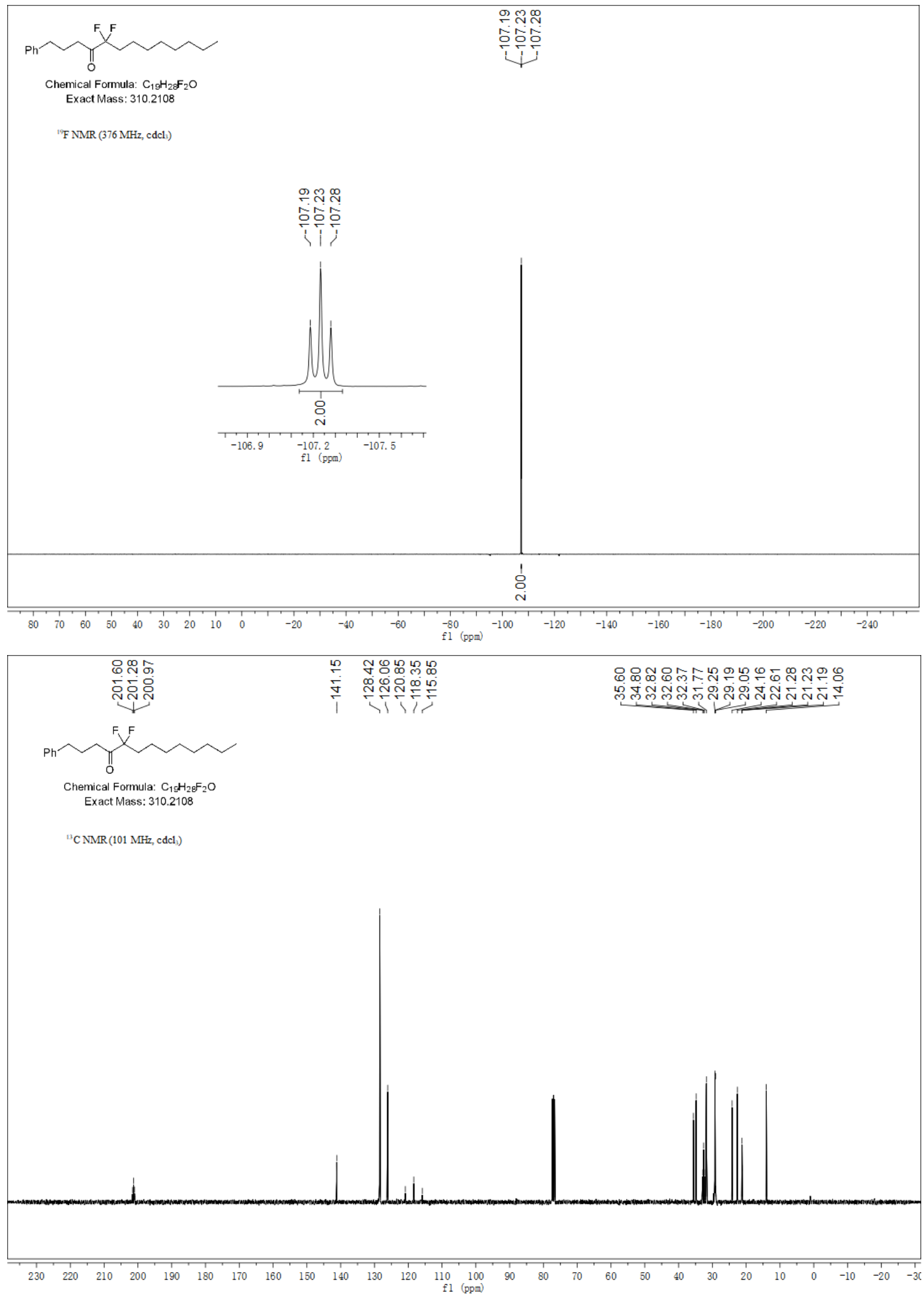
5,5-Difluoro-6-0xo-9-phenylnonyl acetate (3I).
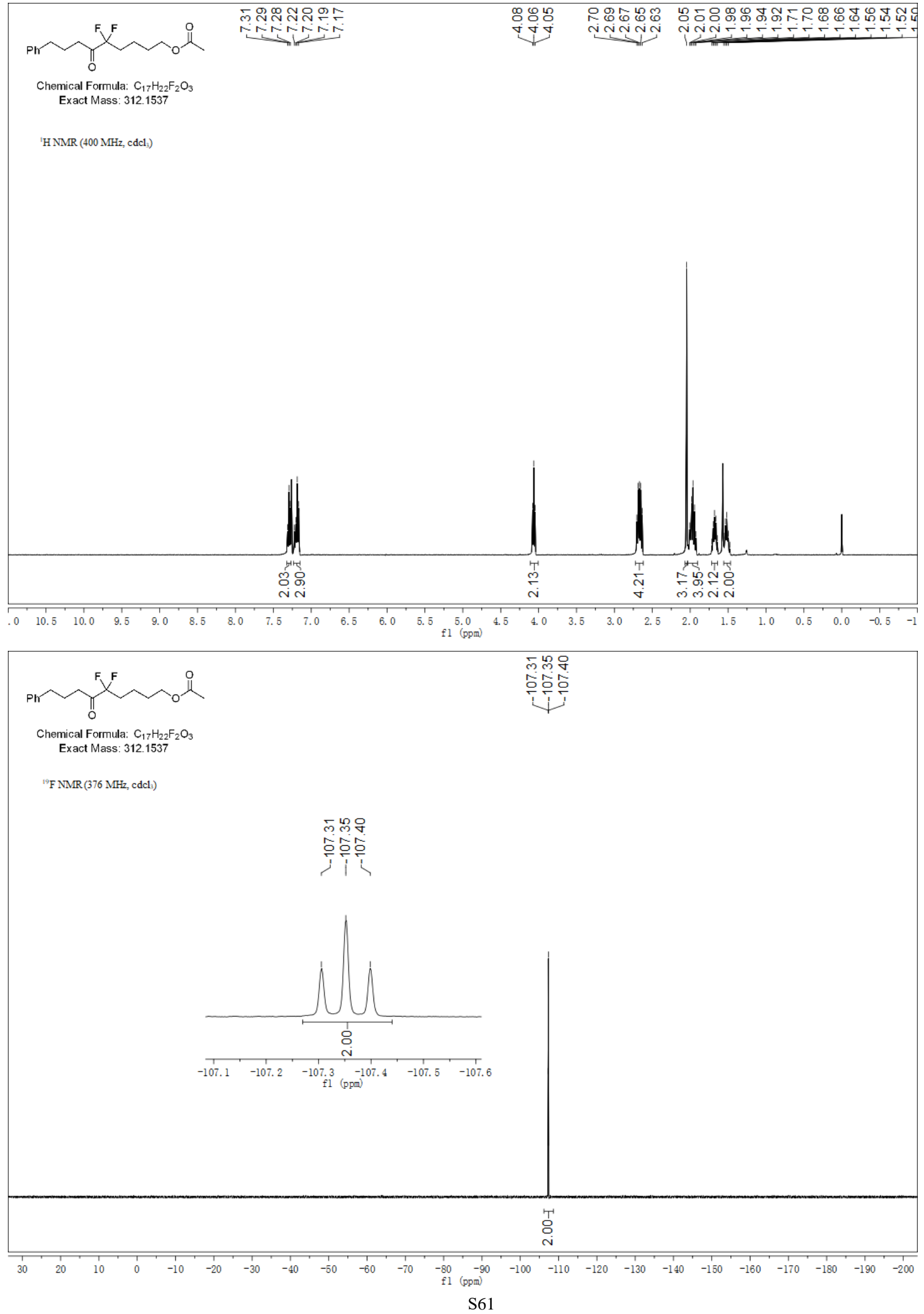


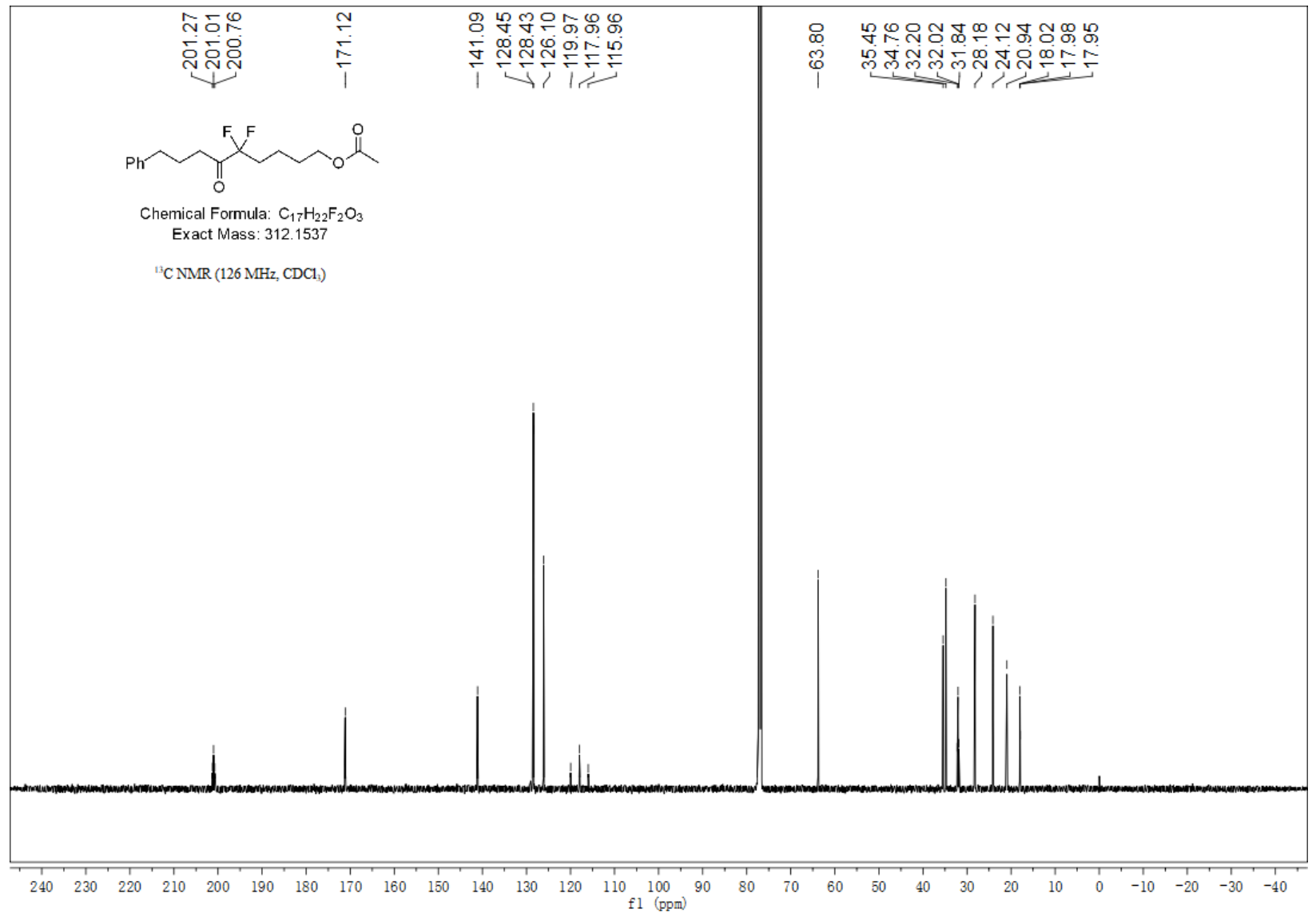

8-((tert-Butyldimethylsilyl)oxy)-5,5-difluoro-1-phenyloctan-4-one (3m).

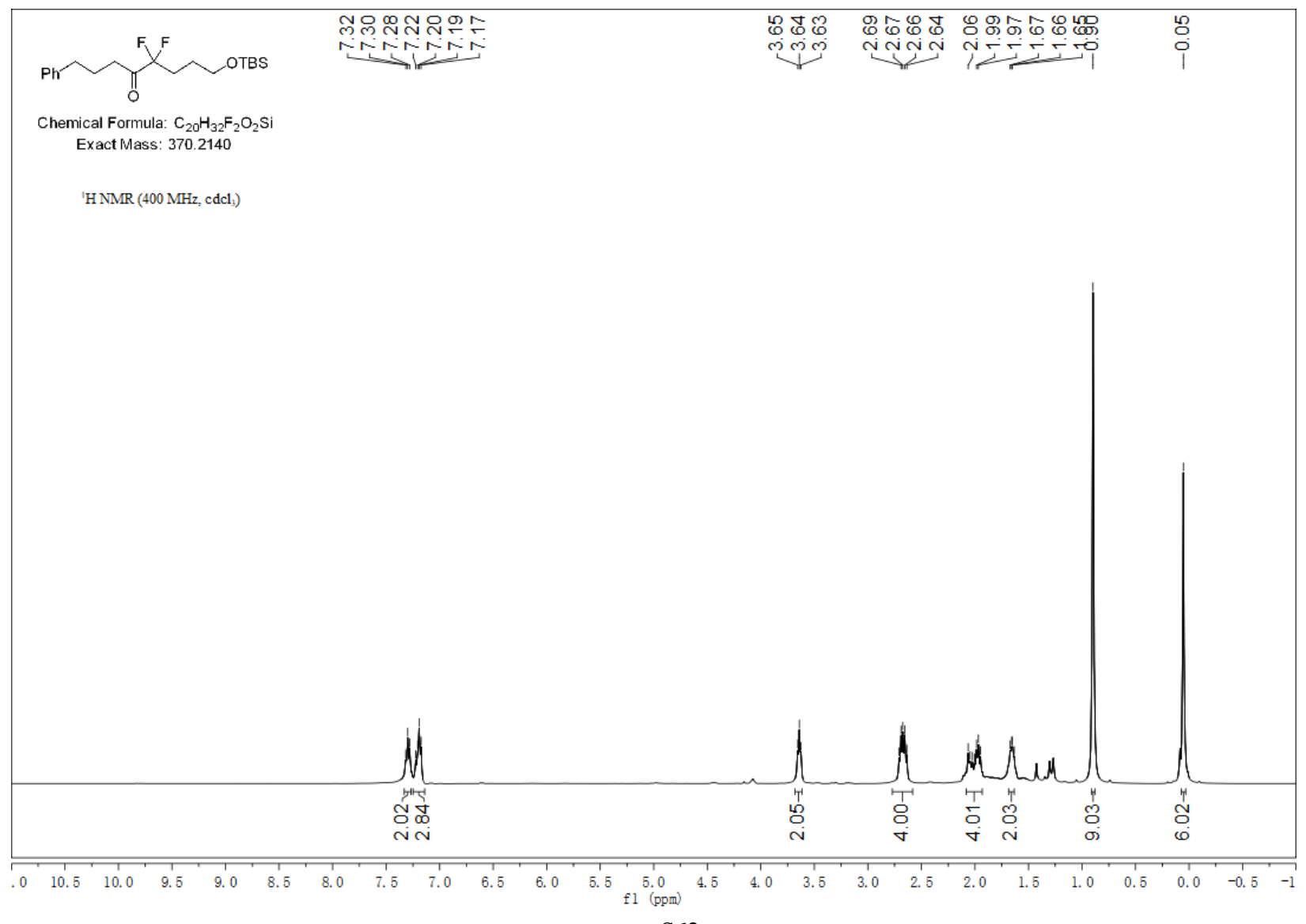



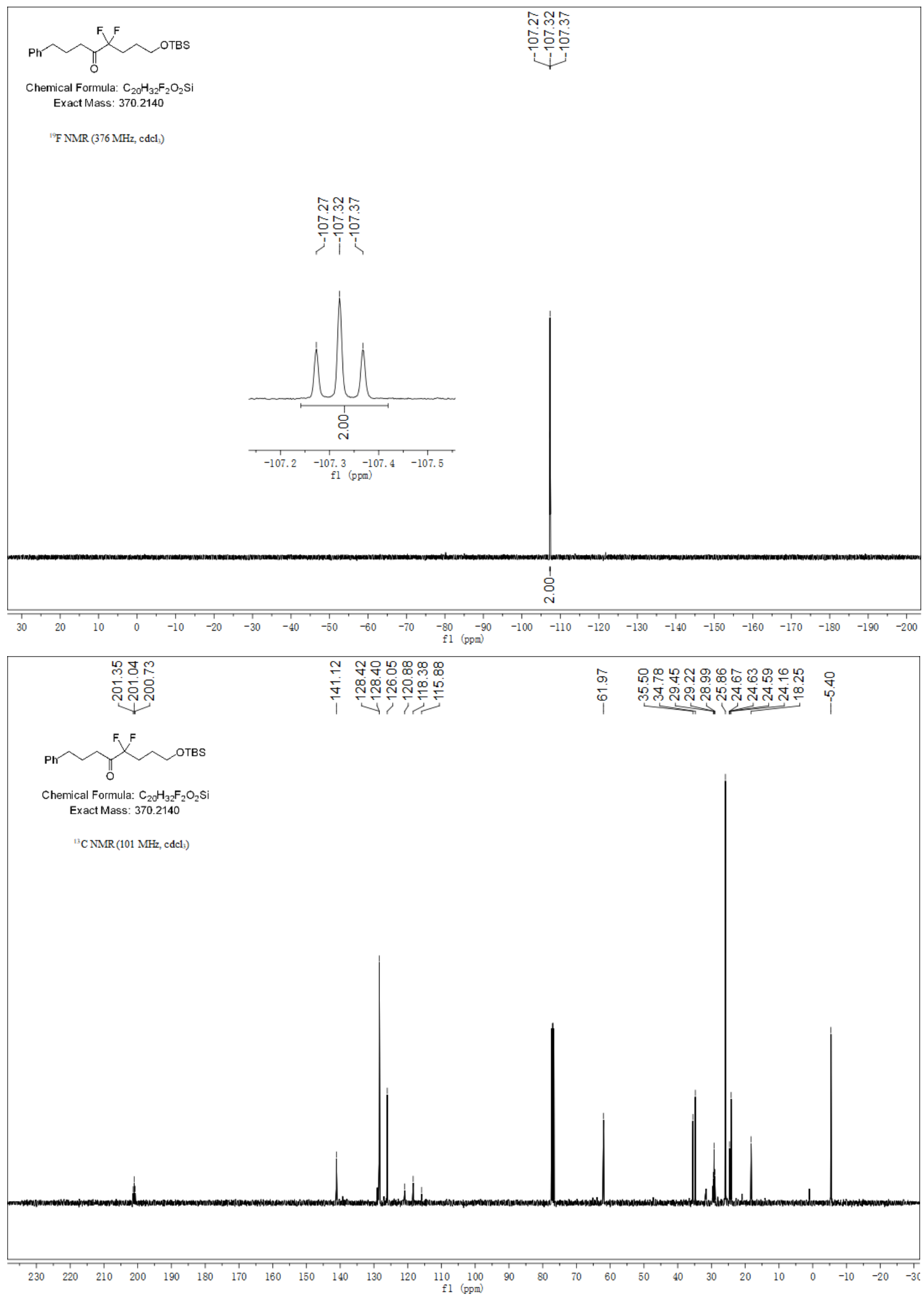


\section{0-Chloro-5,5-difluoro-1-phenyldecan-4-one (3n).}
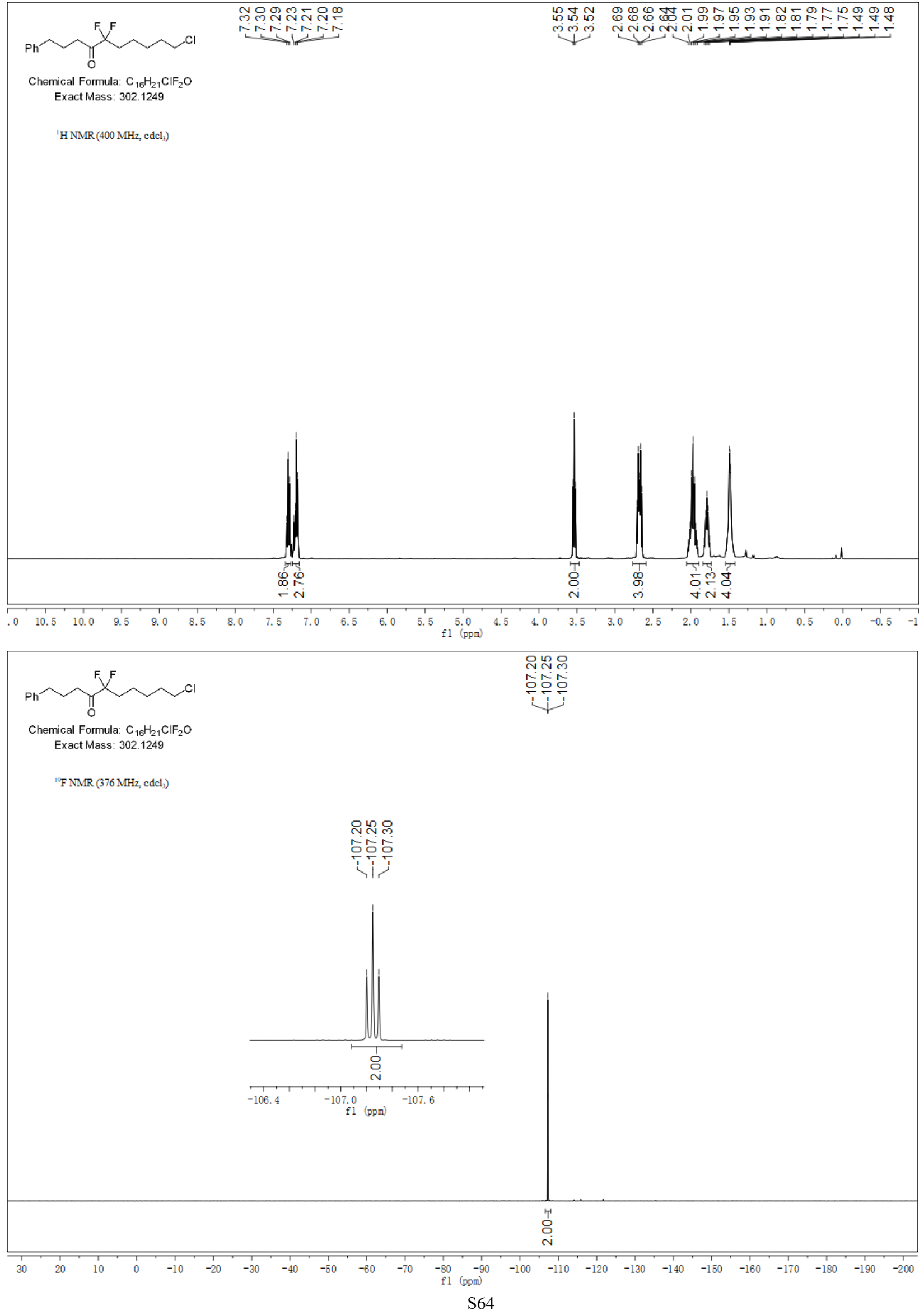


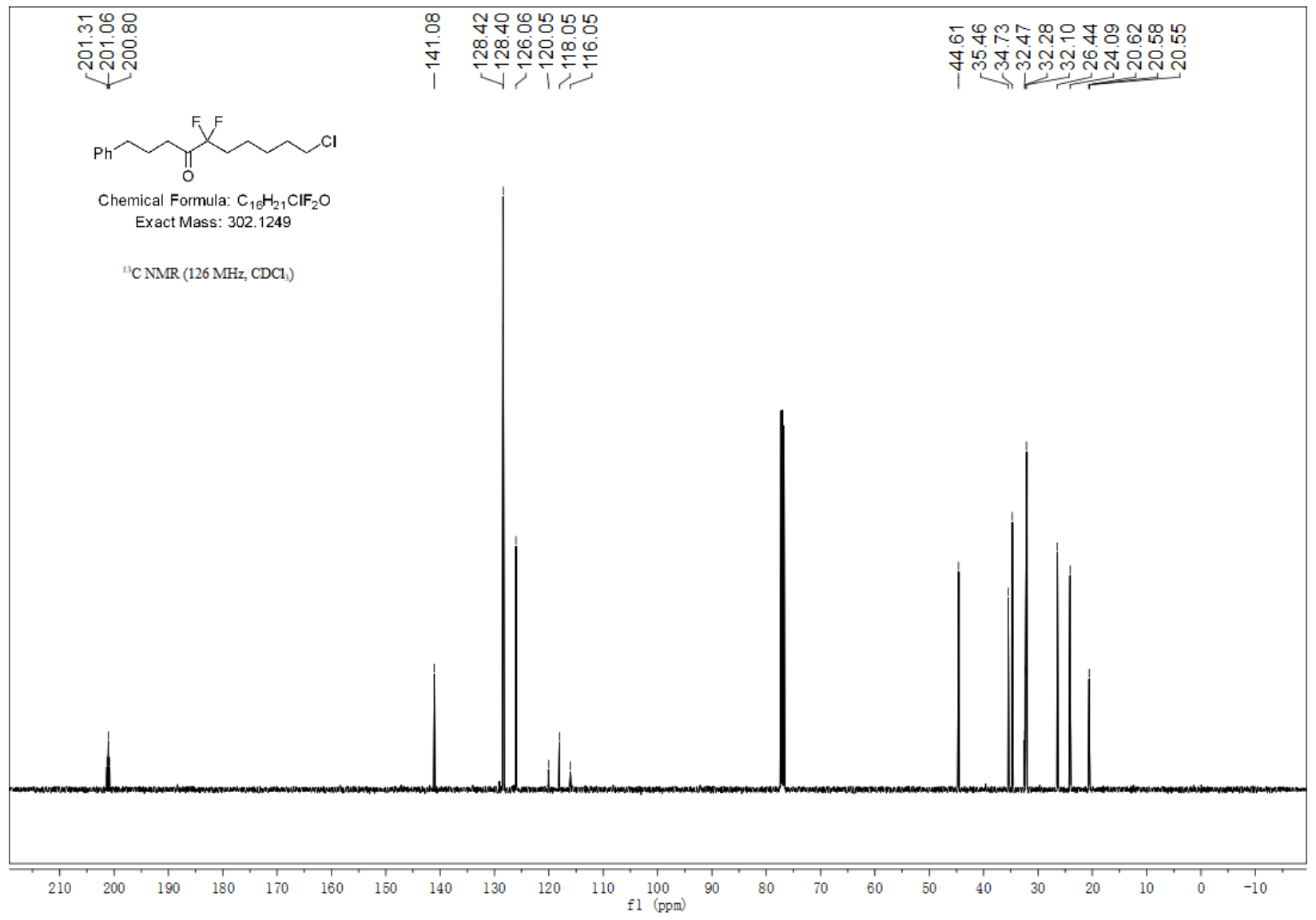

6,6-Difluoro-7-oxo-10-phenyldecanenitrile (3o).

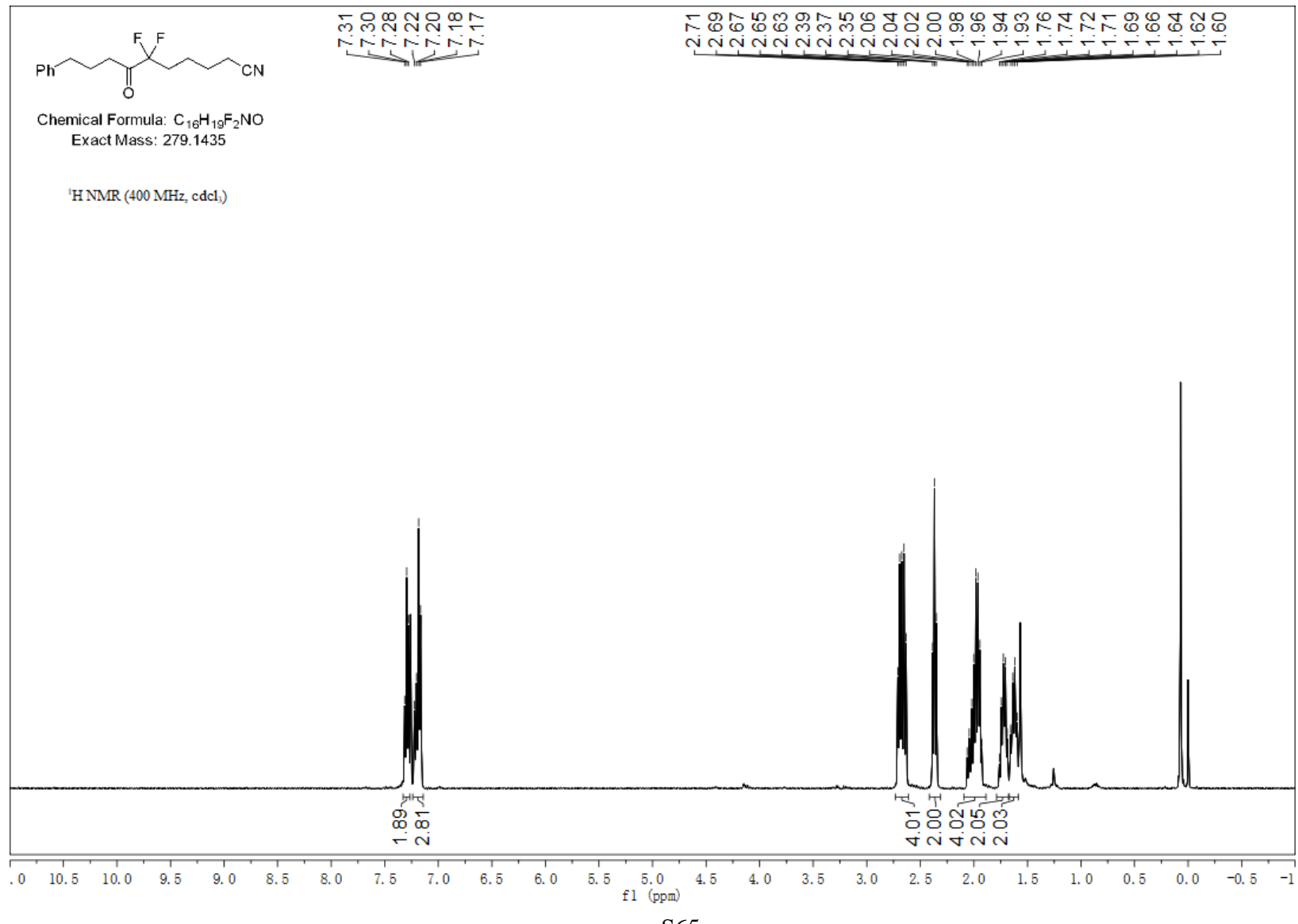



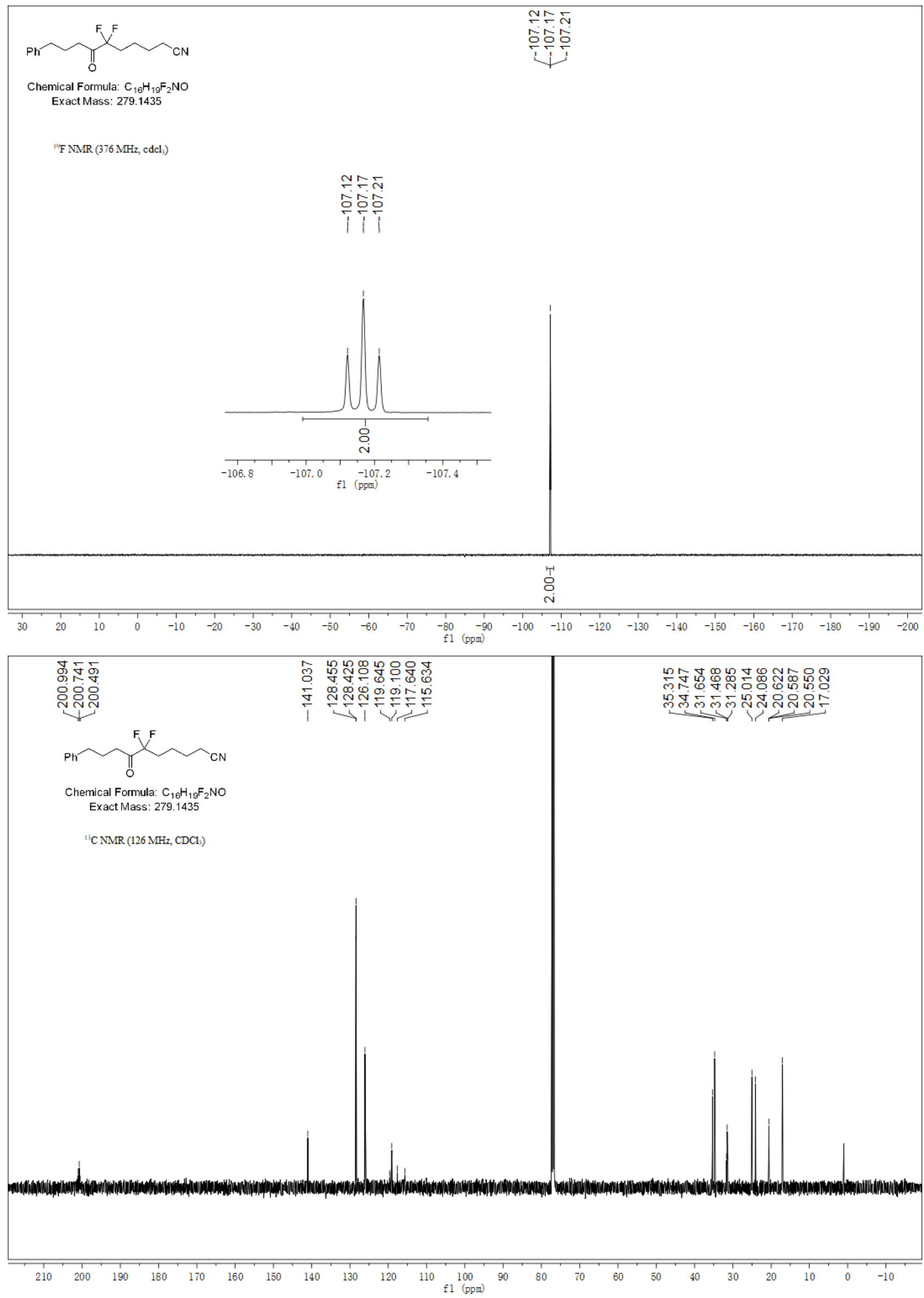
Methyl 4-(2,2-difluoro-3-oxo-6-phenylhexyl)benzoate (3p).
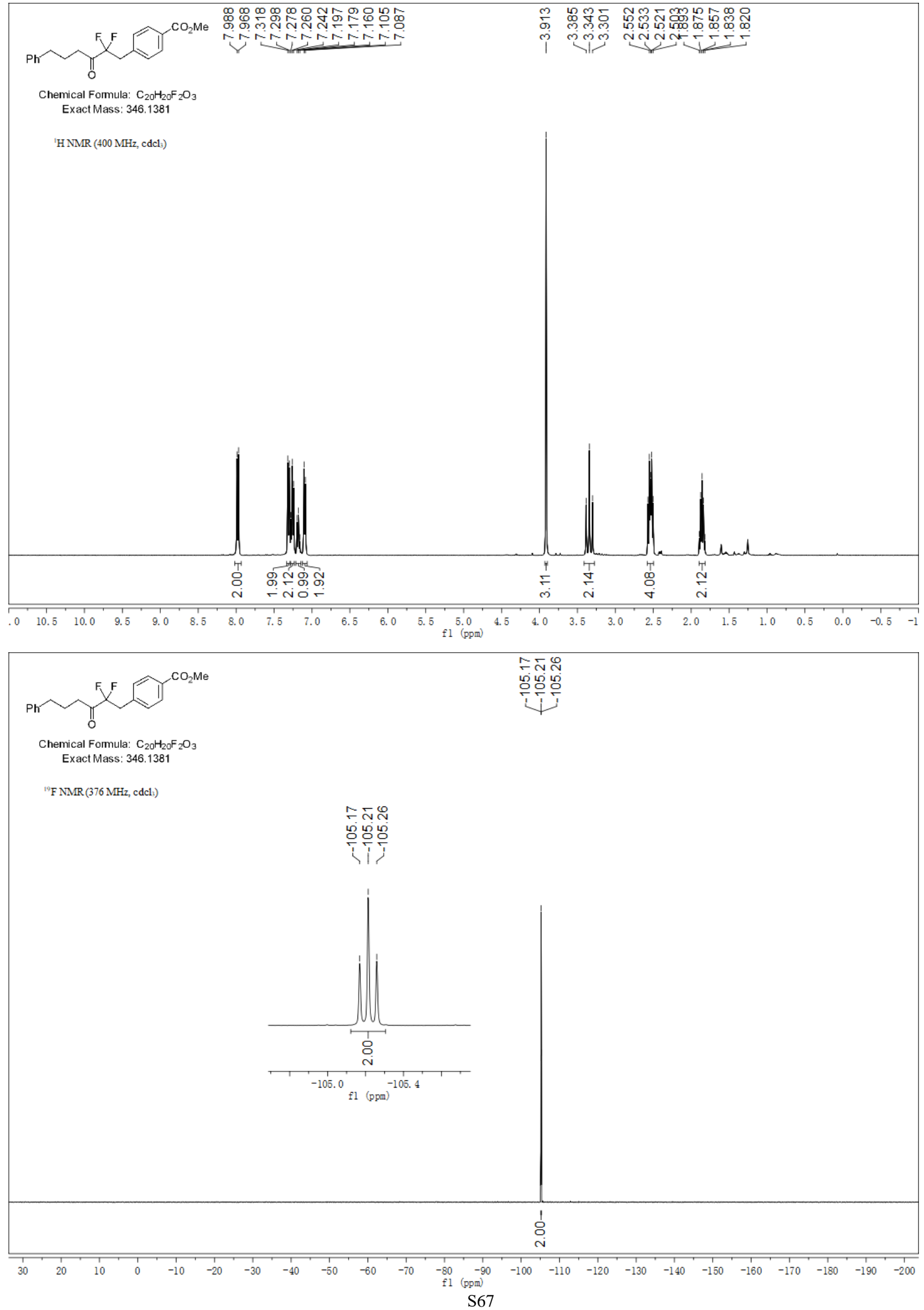


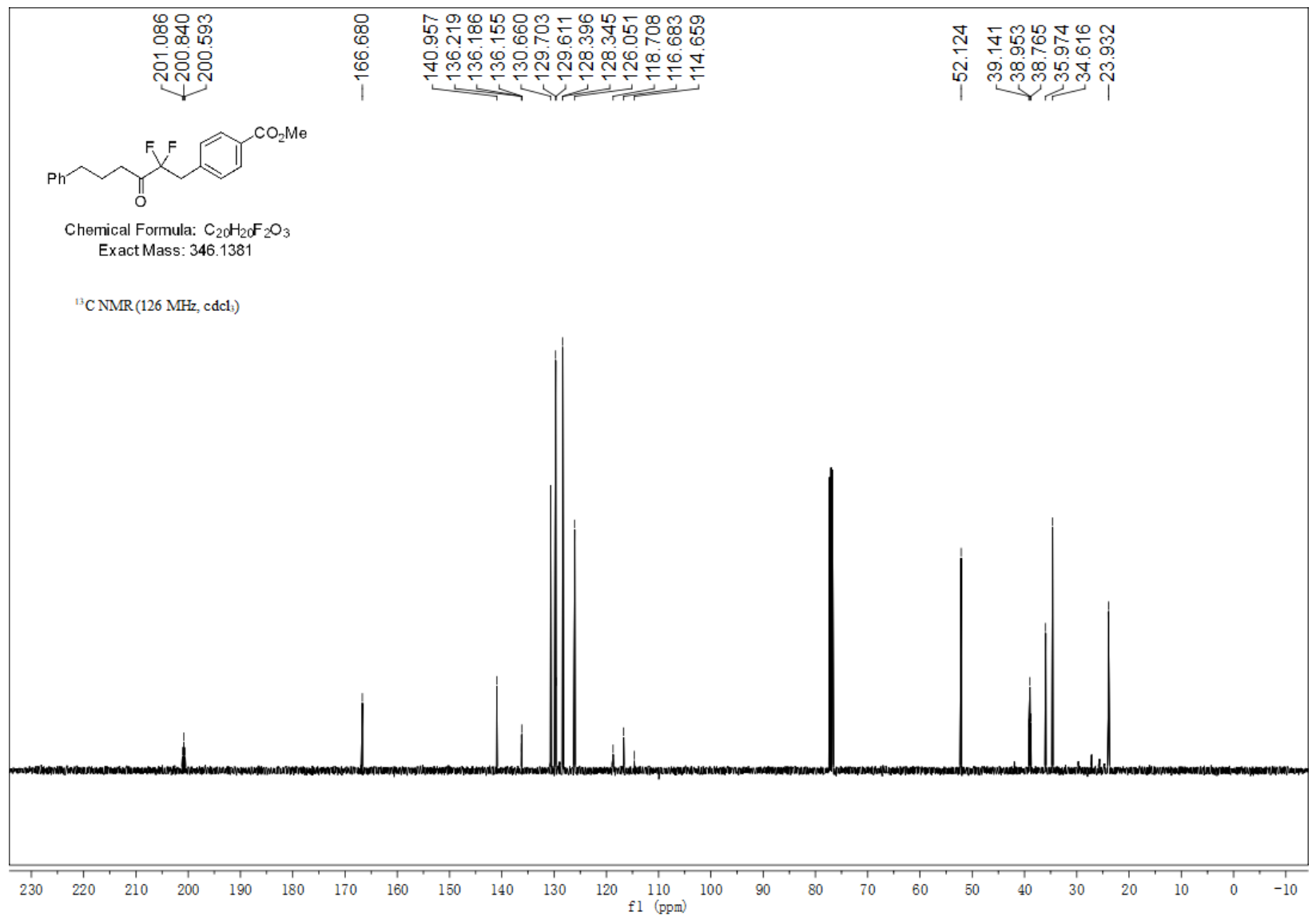

4,4-Difluoro-5-oxo-8-phenyloctyl 4-chlorobenzoate (3q).

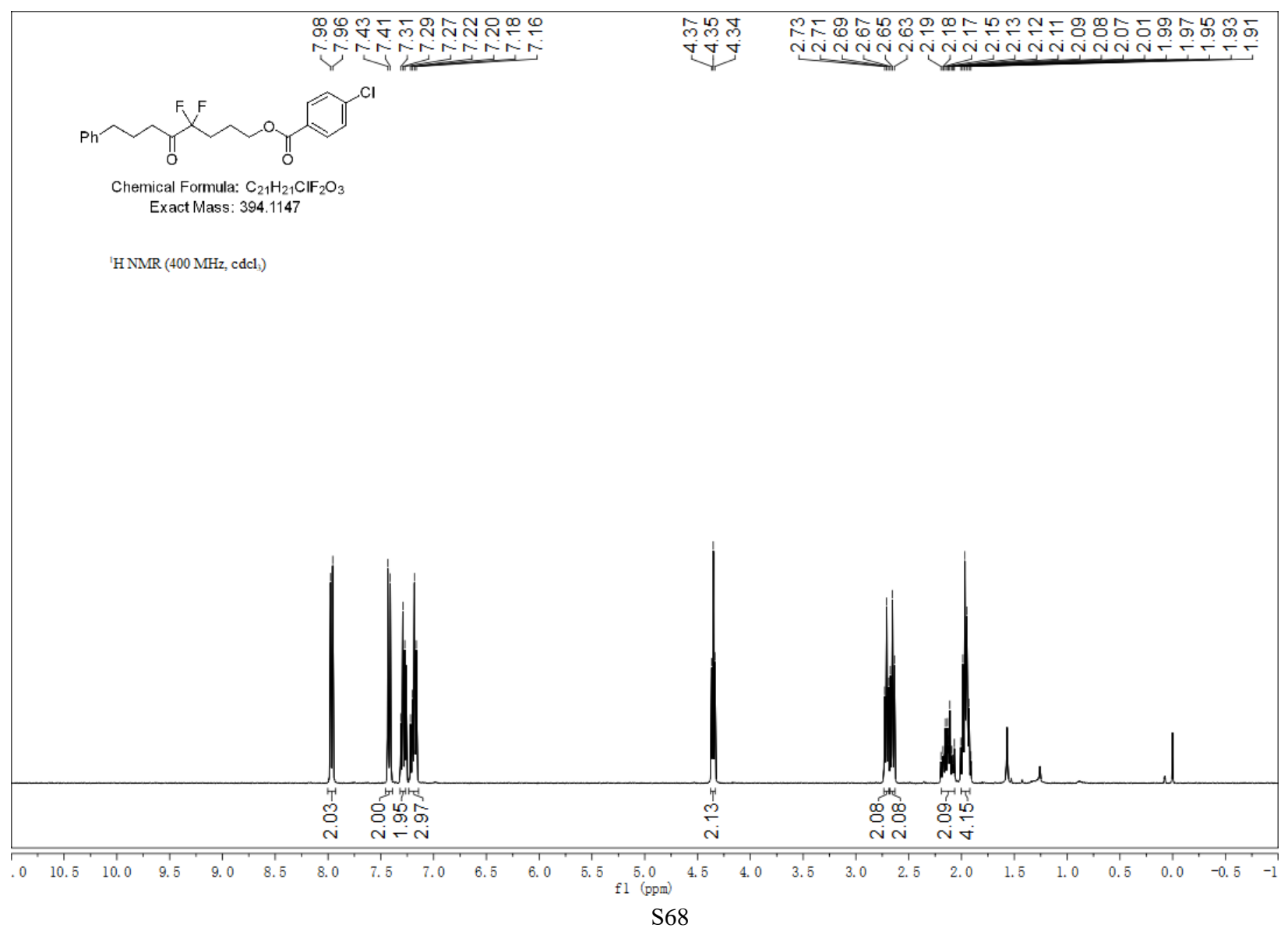



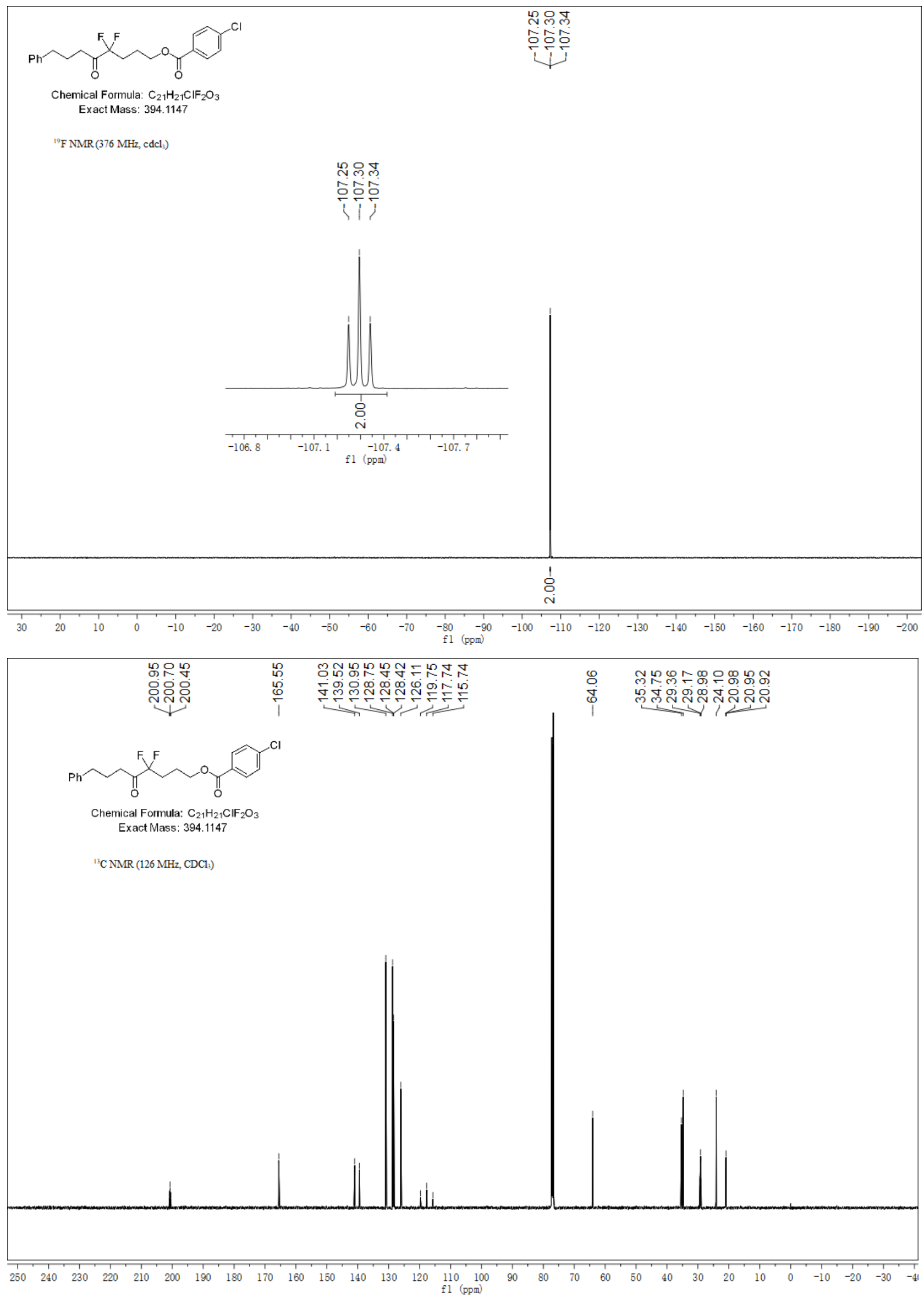
4,4-Difluoro-5-oxo-8-phenyloctyl benzoate (3r).
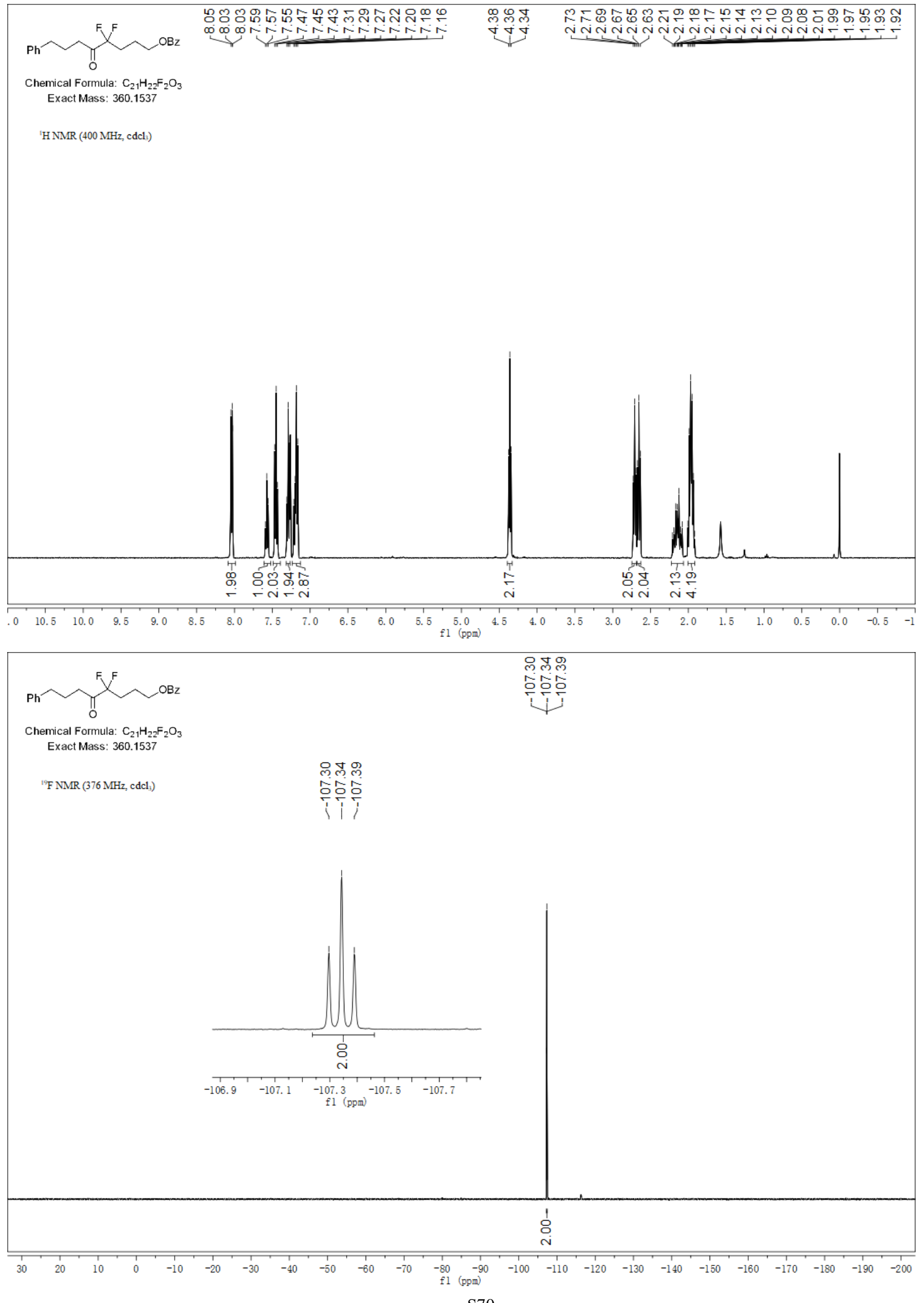


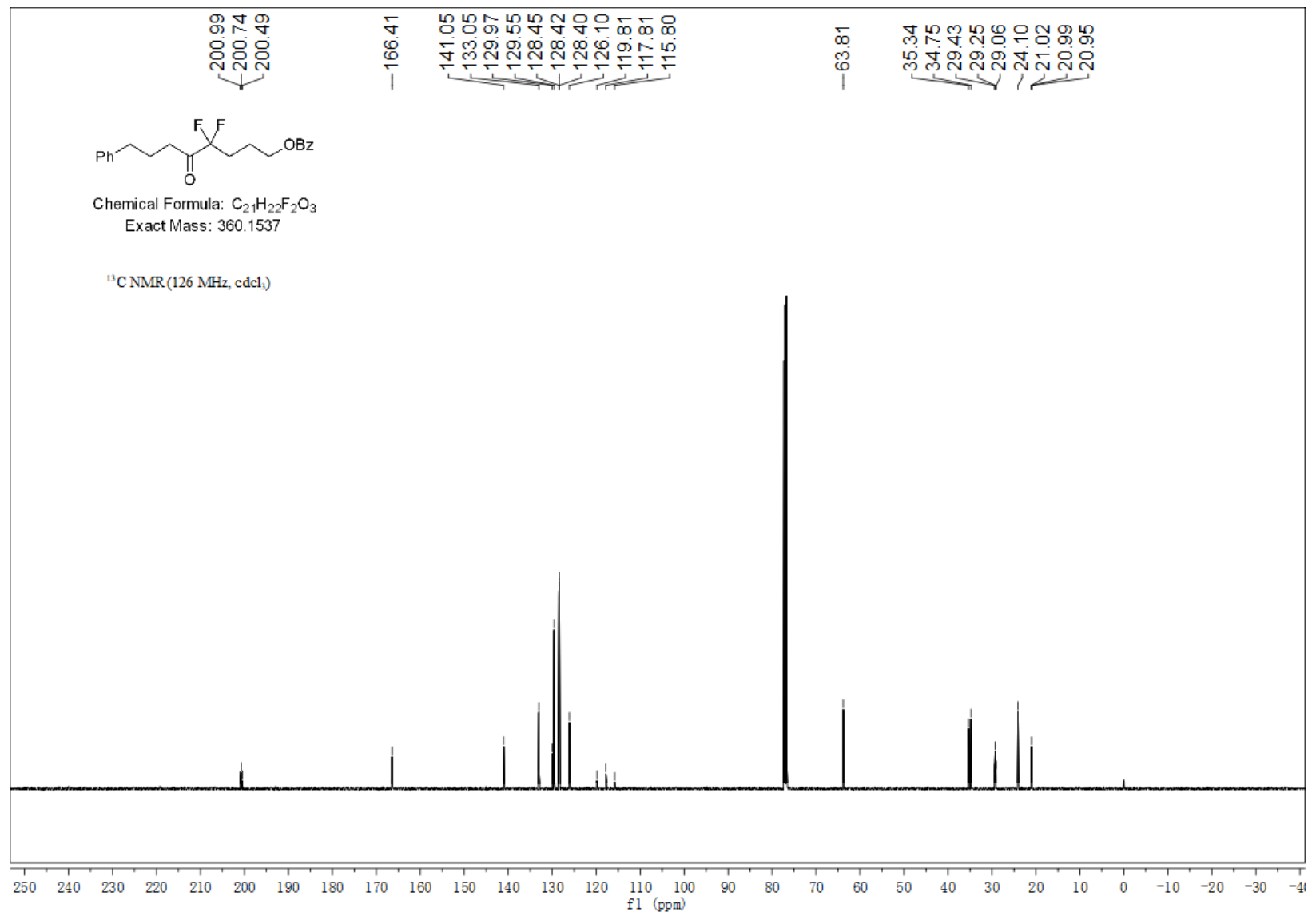

4,4-Difluoro-8-methoxy-5-oxooctyl benzoate (3s).

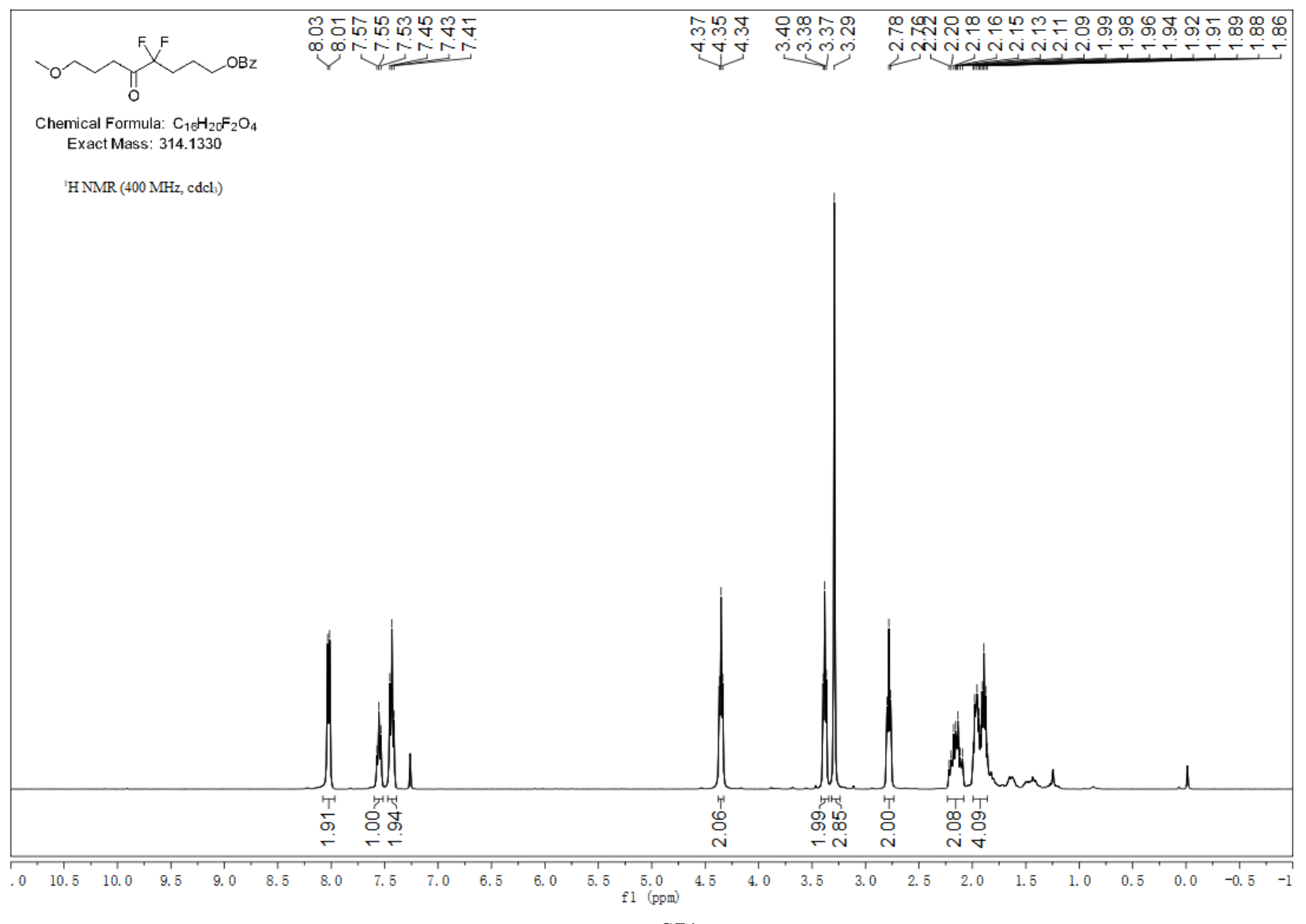



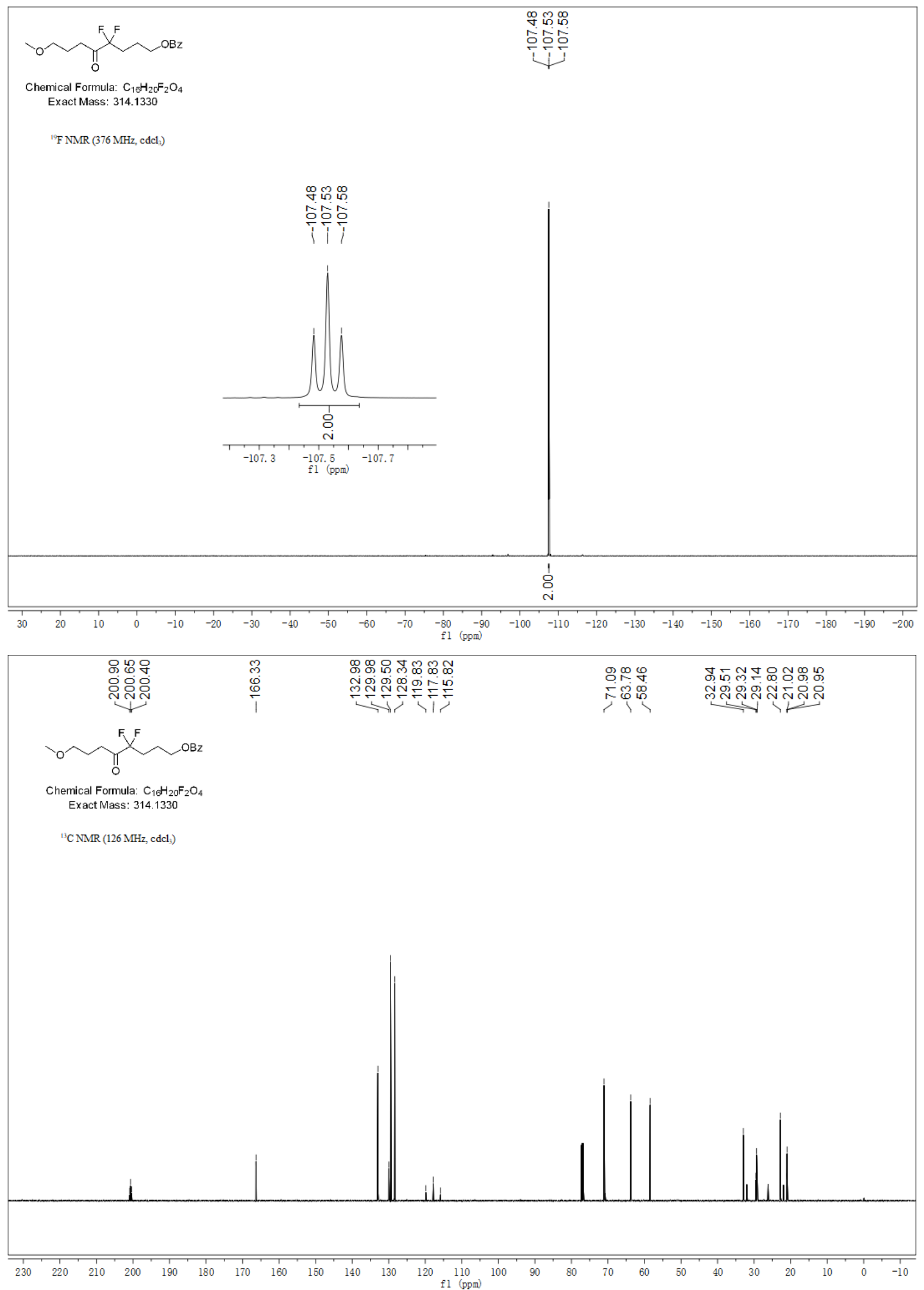
9-(tert-Butoxy)-4,4-difluoro-5,9-dioxononyl benzoate (3t).
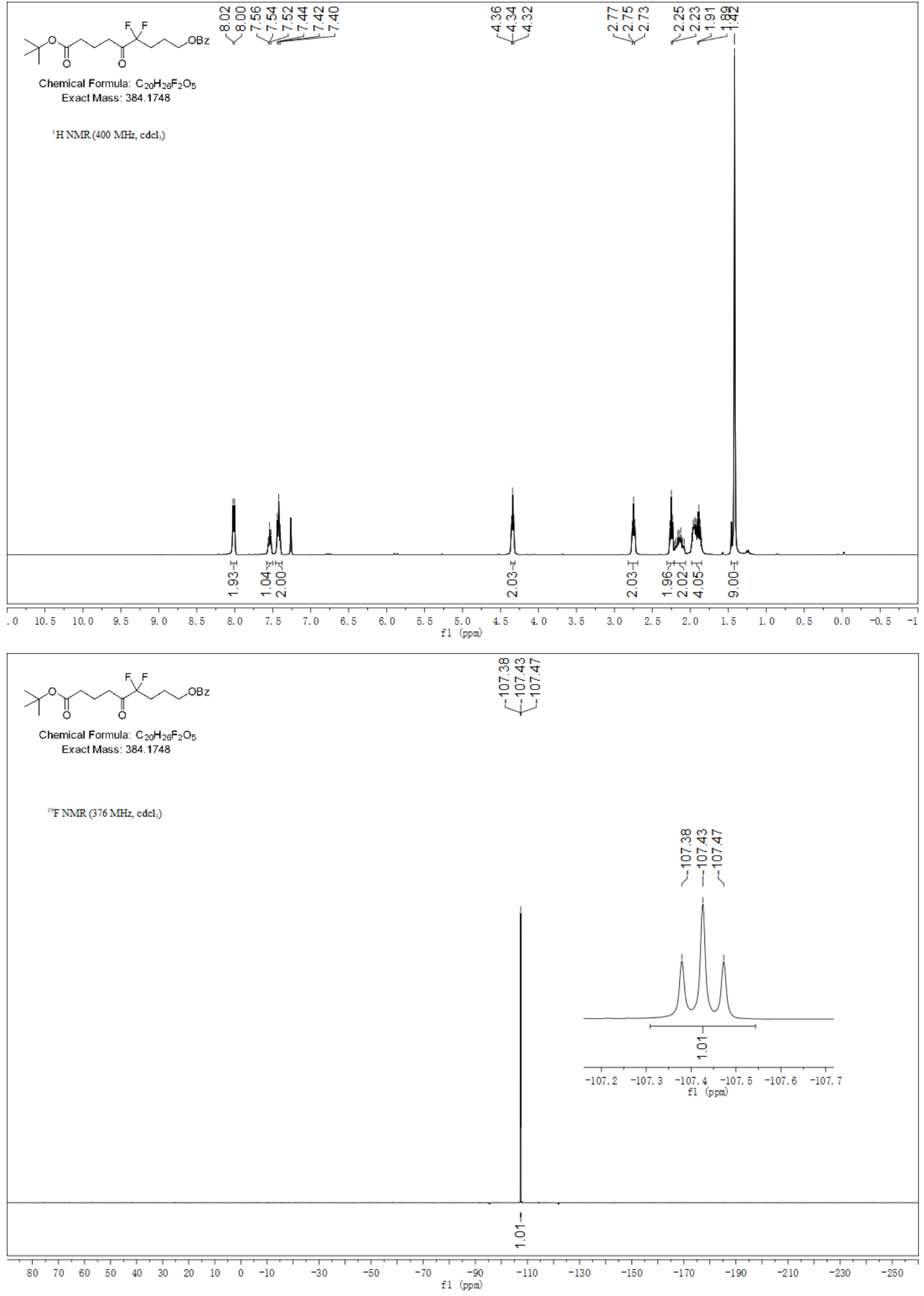


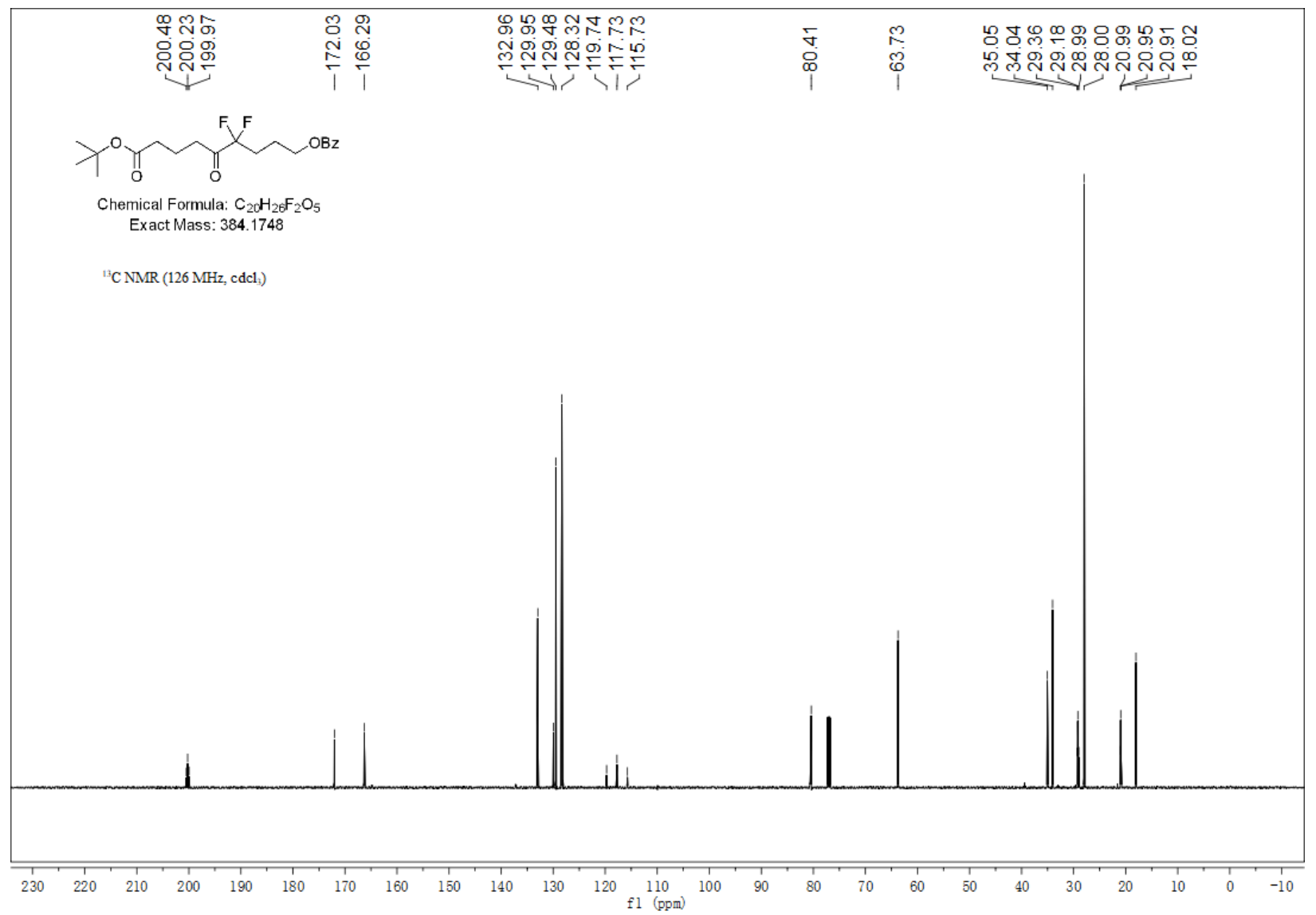

Methyl 4-(7-(tert-butoxy)-2,2-difluoro-3,7-dioxoheptyl)benzoate (3u).

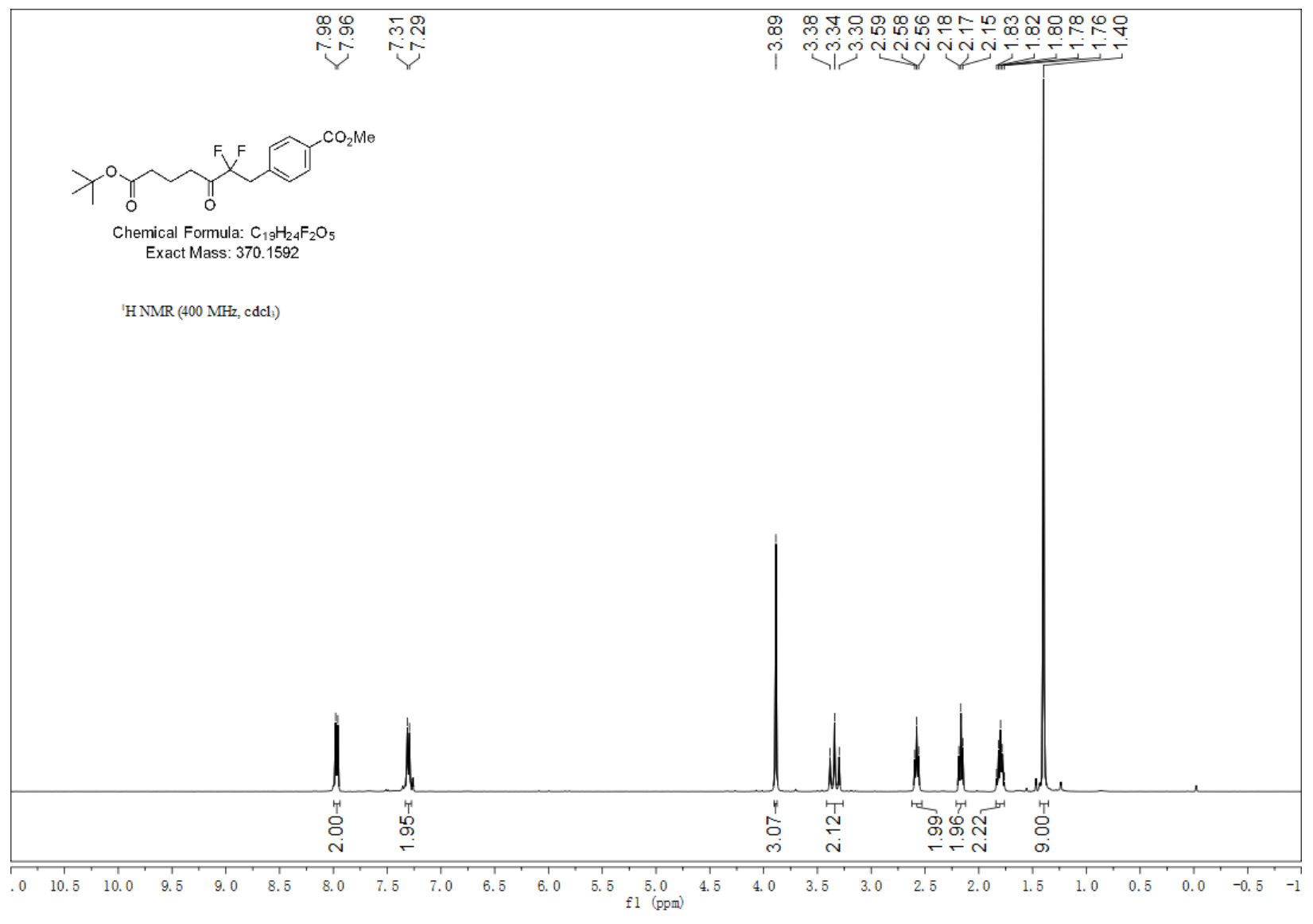



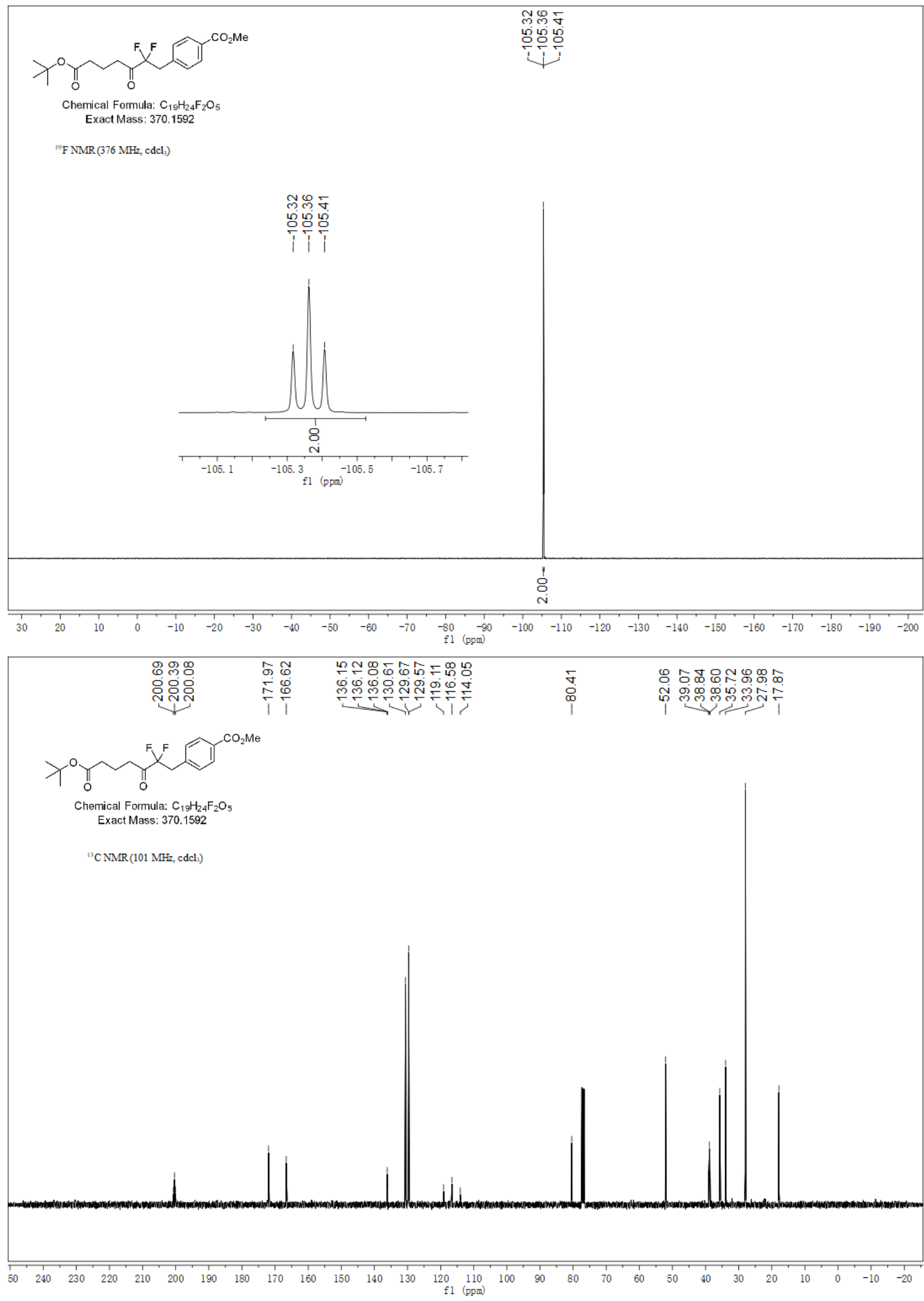
1-((tert-Butyldimethylsilyl)oxy)-2,2-difluoro-6-phenylhexan-3-one (3v).
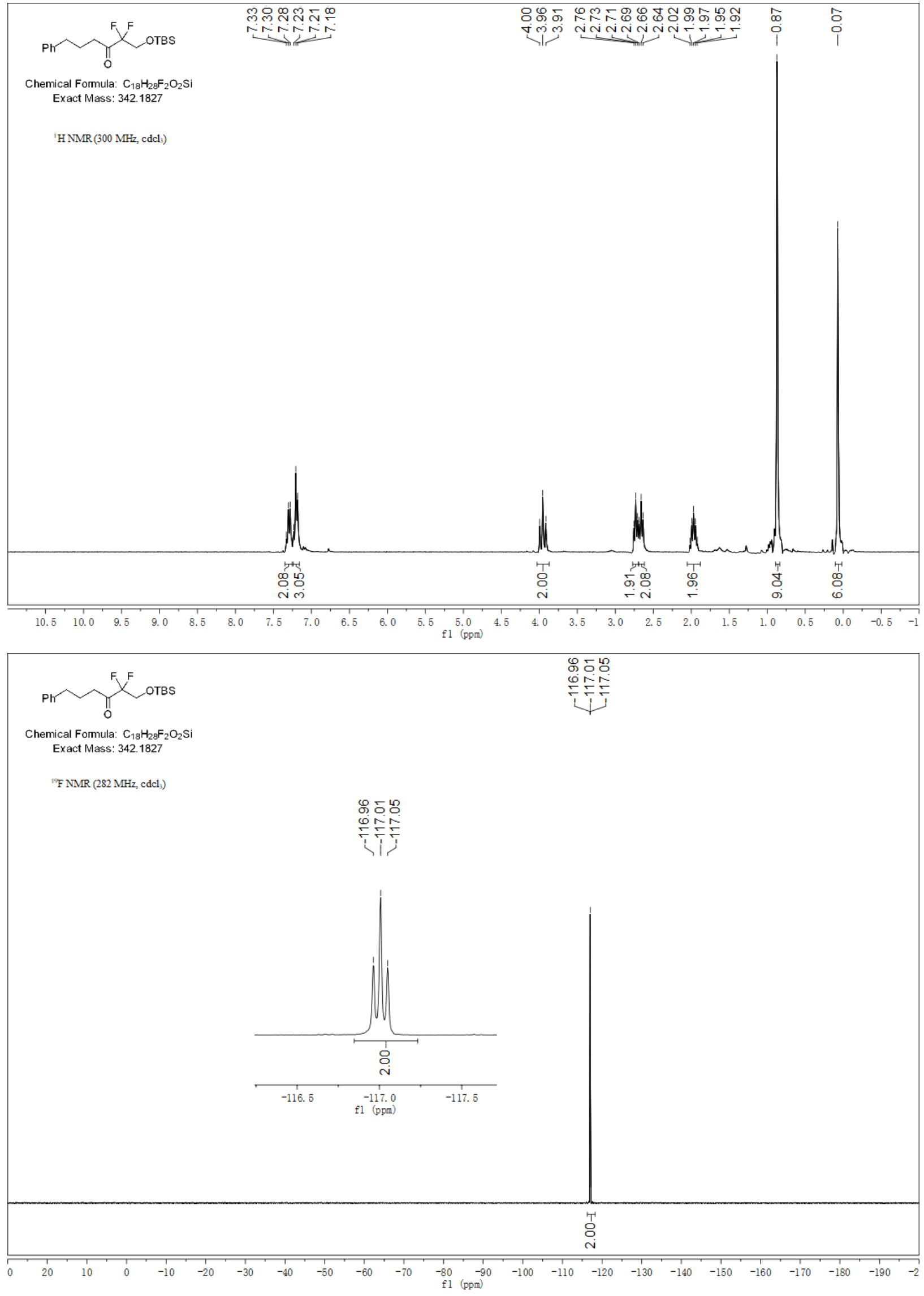


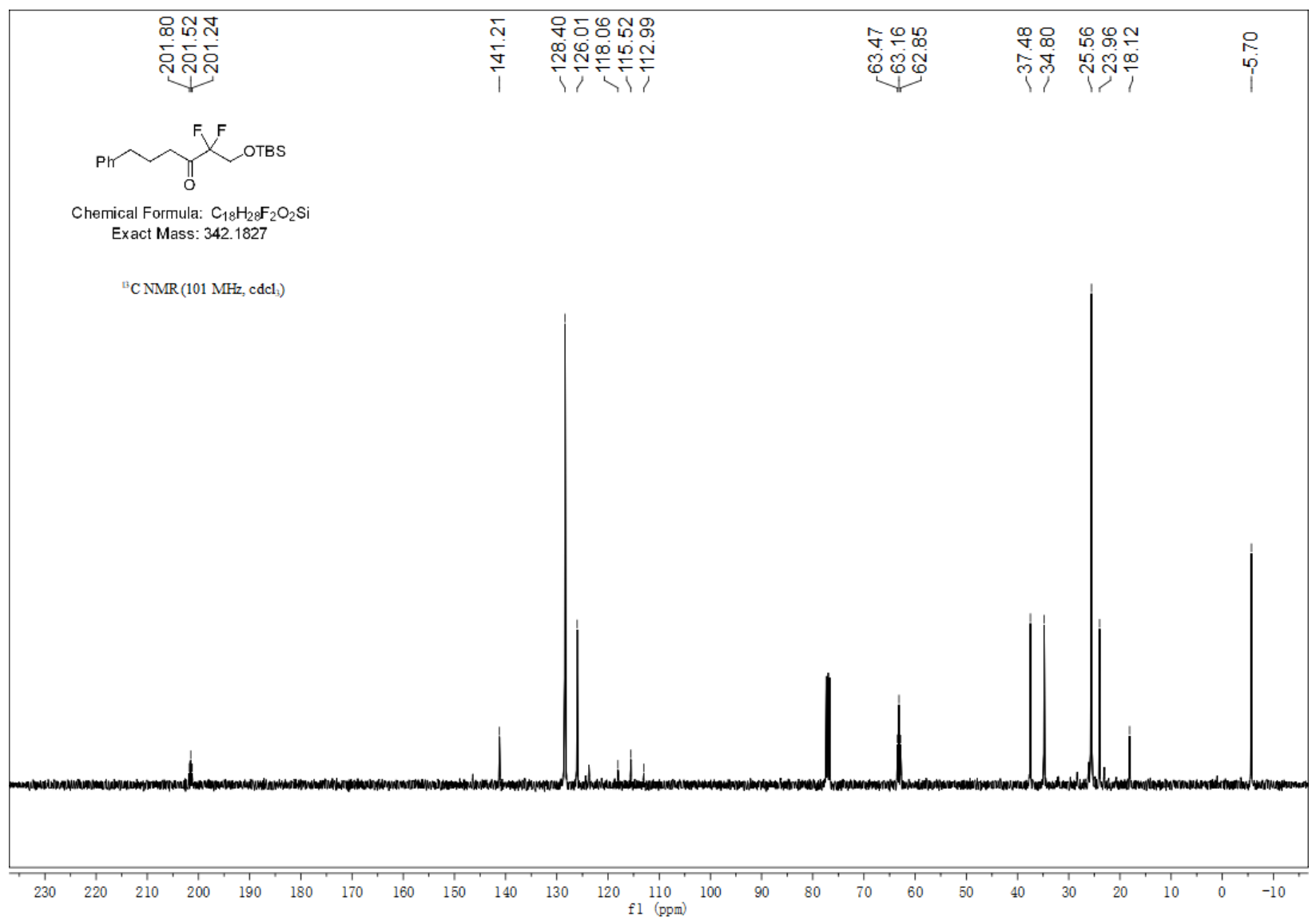

tert-Butyl-7-((tert-butyldimethylsilyl)oxy)-6,6-difluoro-5-oxoheptanoate (3w).

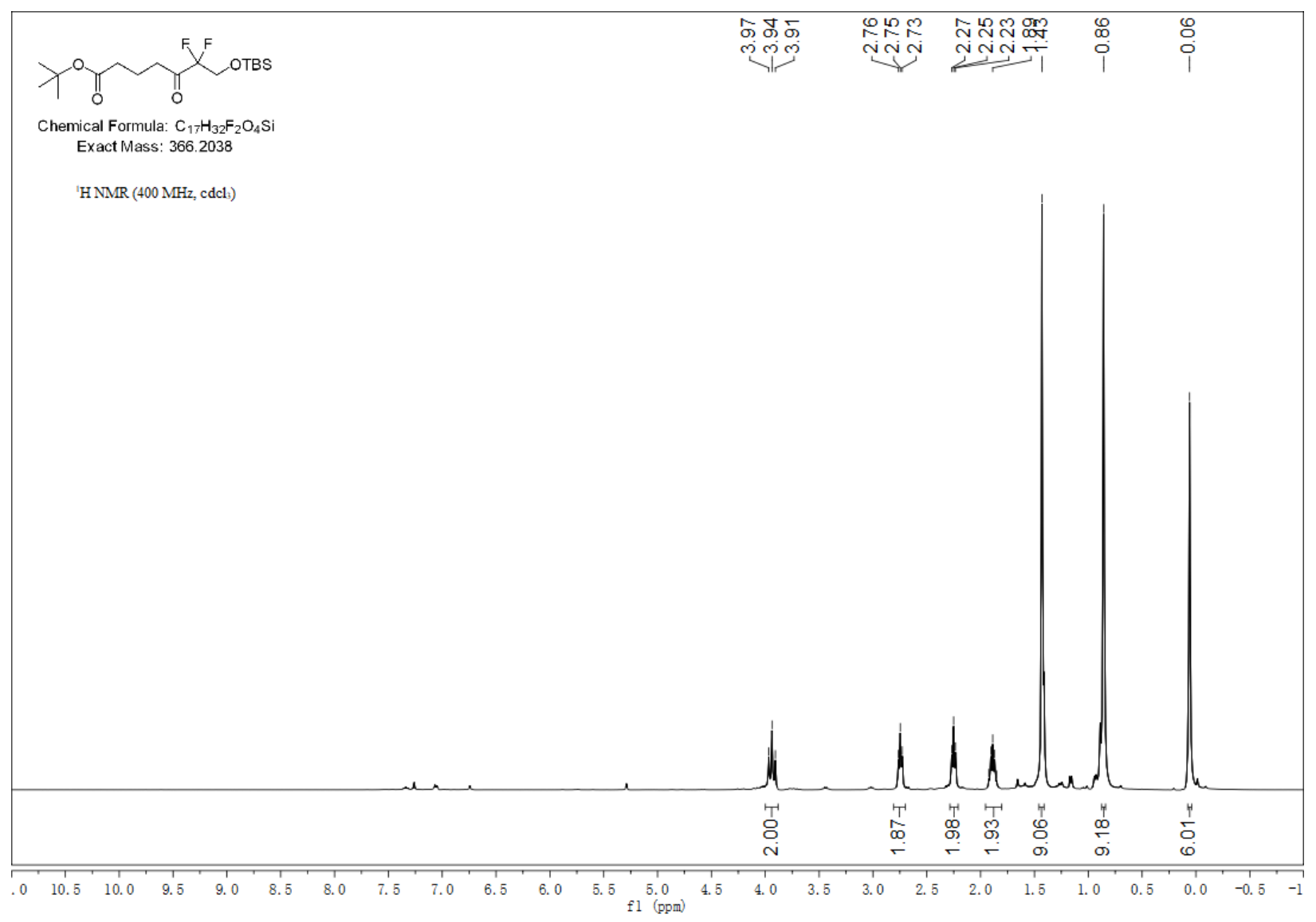



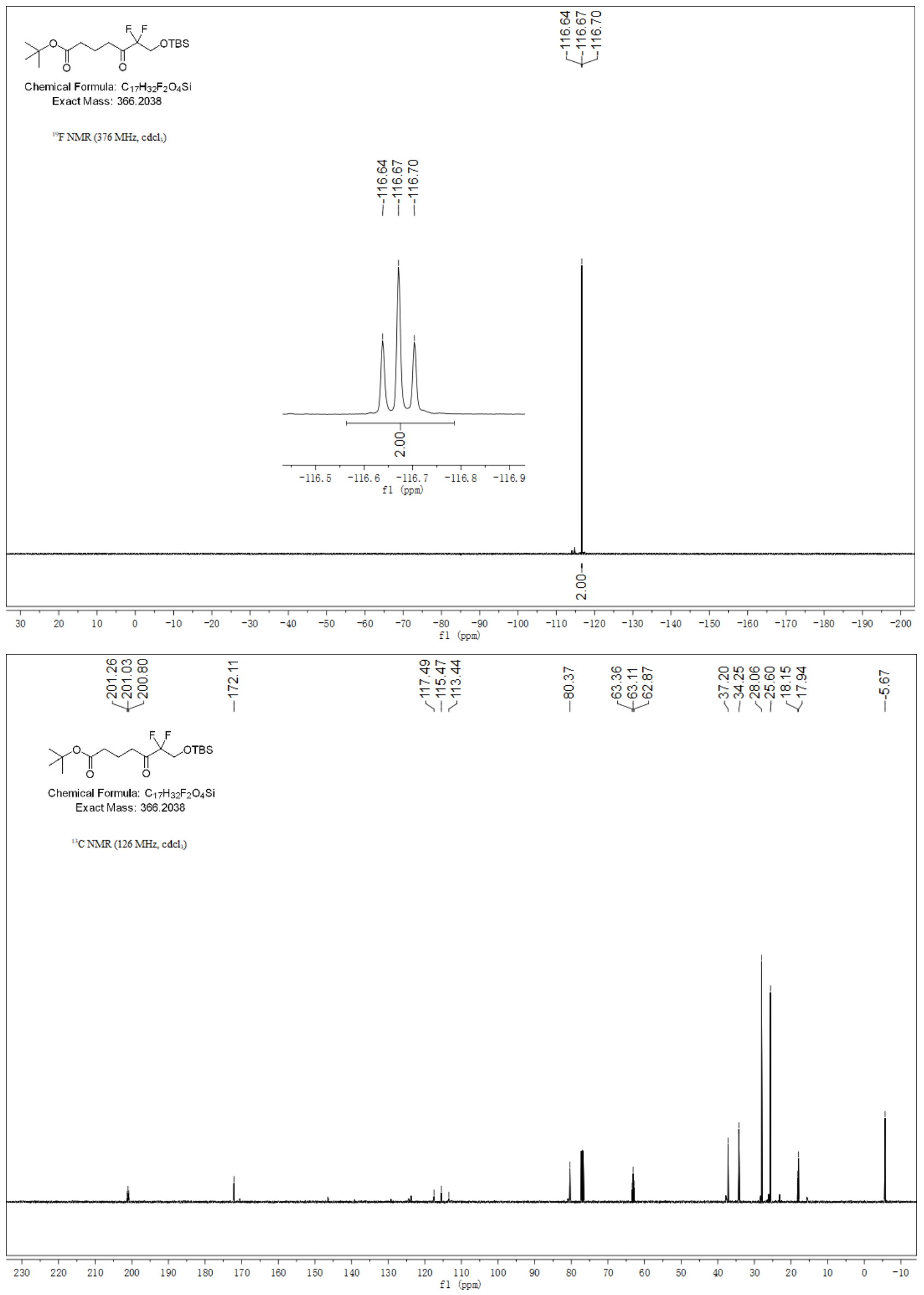
$N, N$-Diethyl-2,2-difluoro-3-oxo-6-phenylhexanamide (7a).
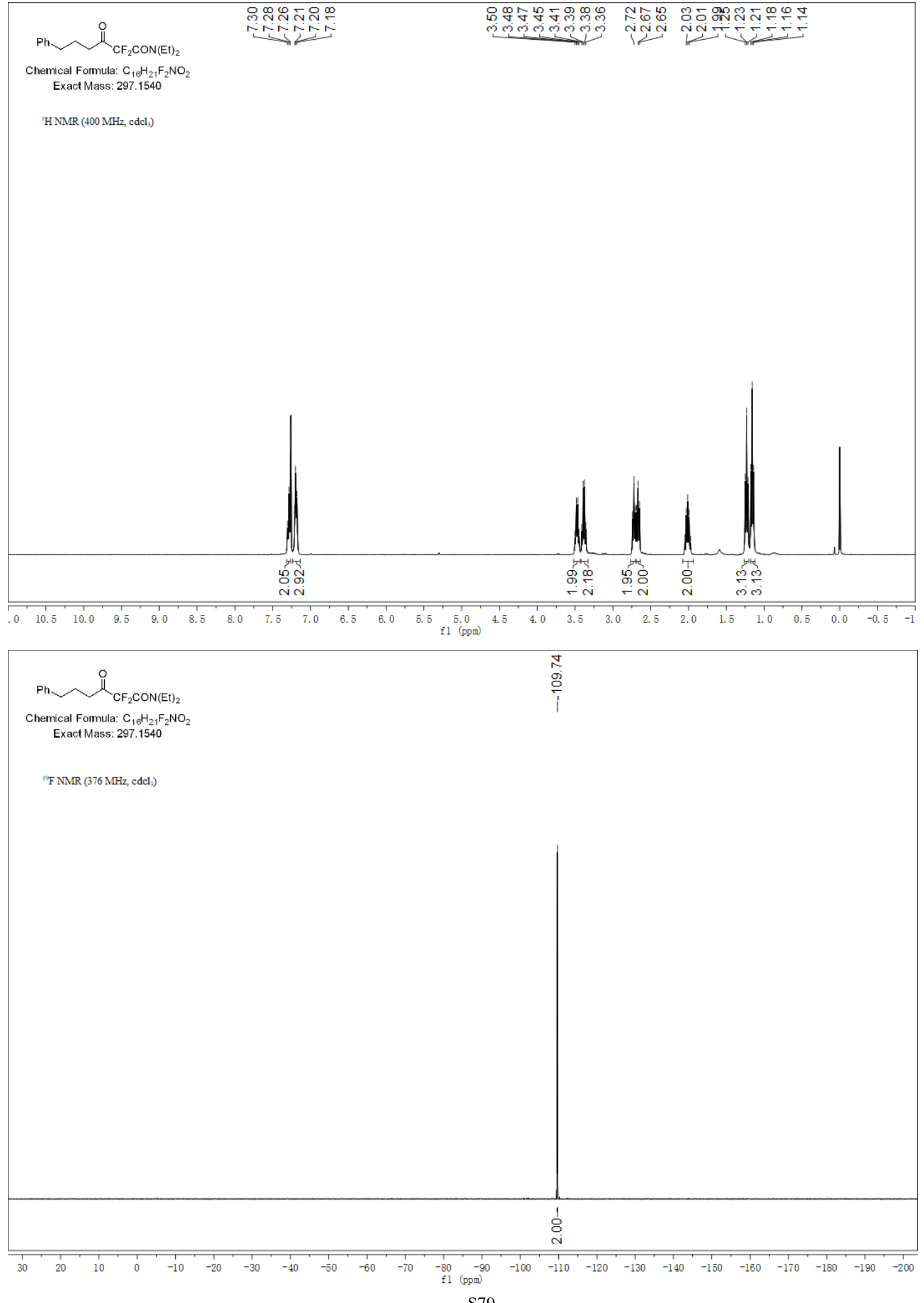


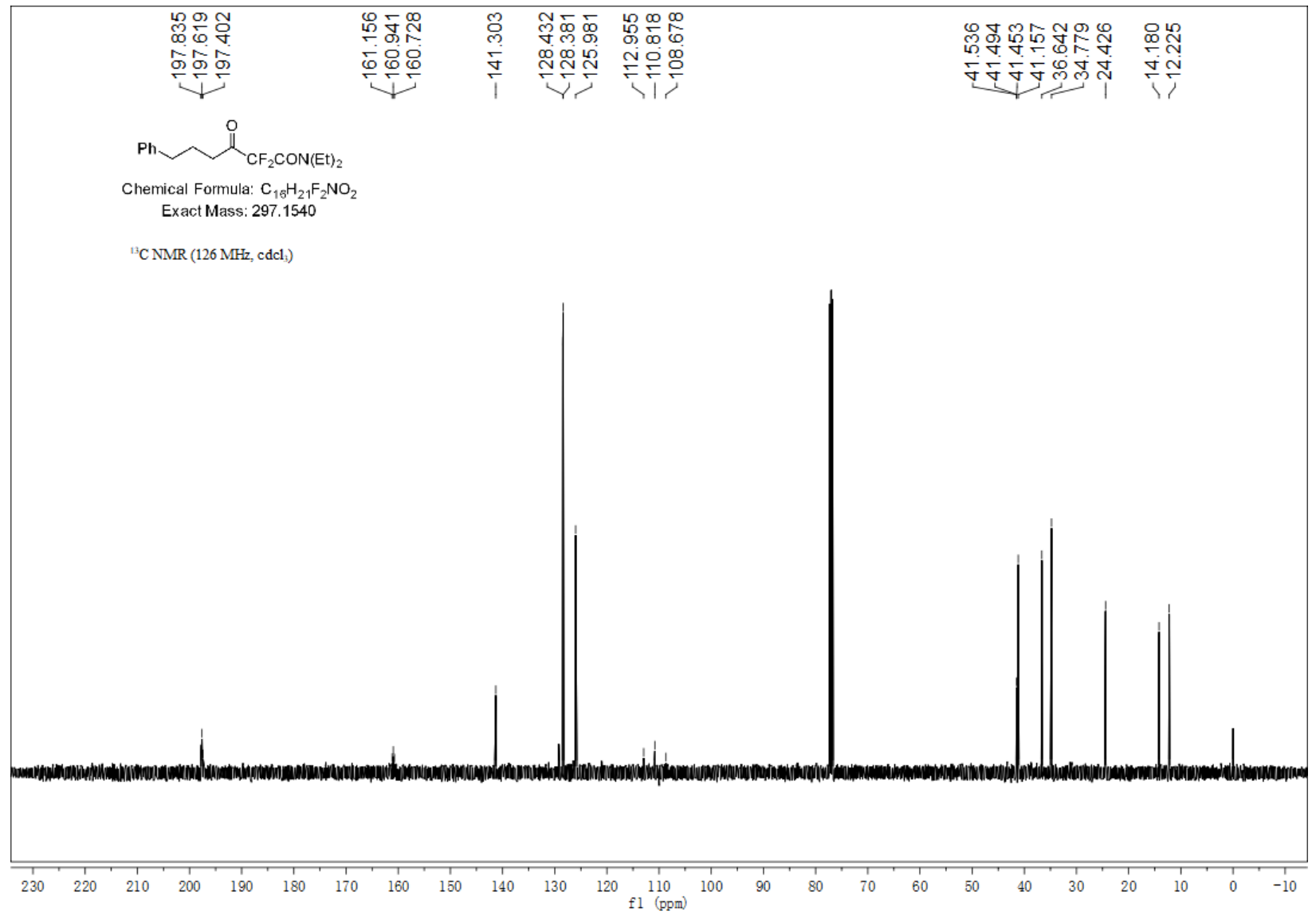

6-(Benzyloxy)- $N, N$-diethyl-2,2-difluoro-3-oxohexanamide (7b).

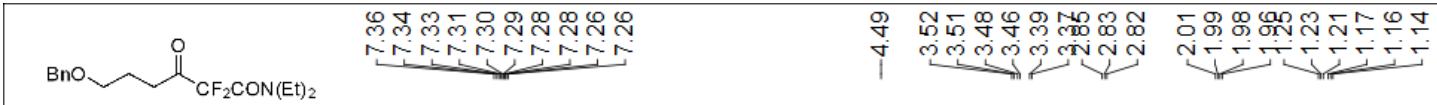

Chemical Formula: $\mathrm{C}_{17} \mathrm{H}_{23} \mathrm{~F}_{2} \mathrm{NO}_{3}$

Exact Mass: 327.1646

'H NMR ( $\left.400 \mathrm{MHz}, \mathrm{cdcl}_{2}\right)$

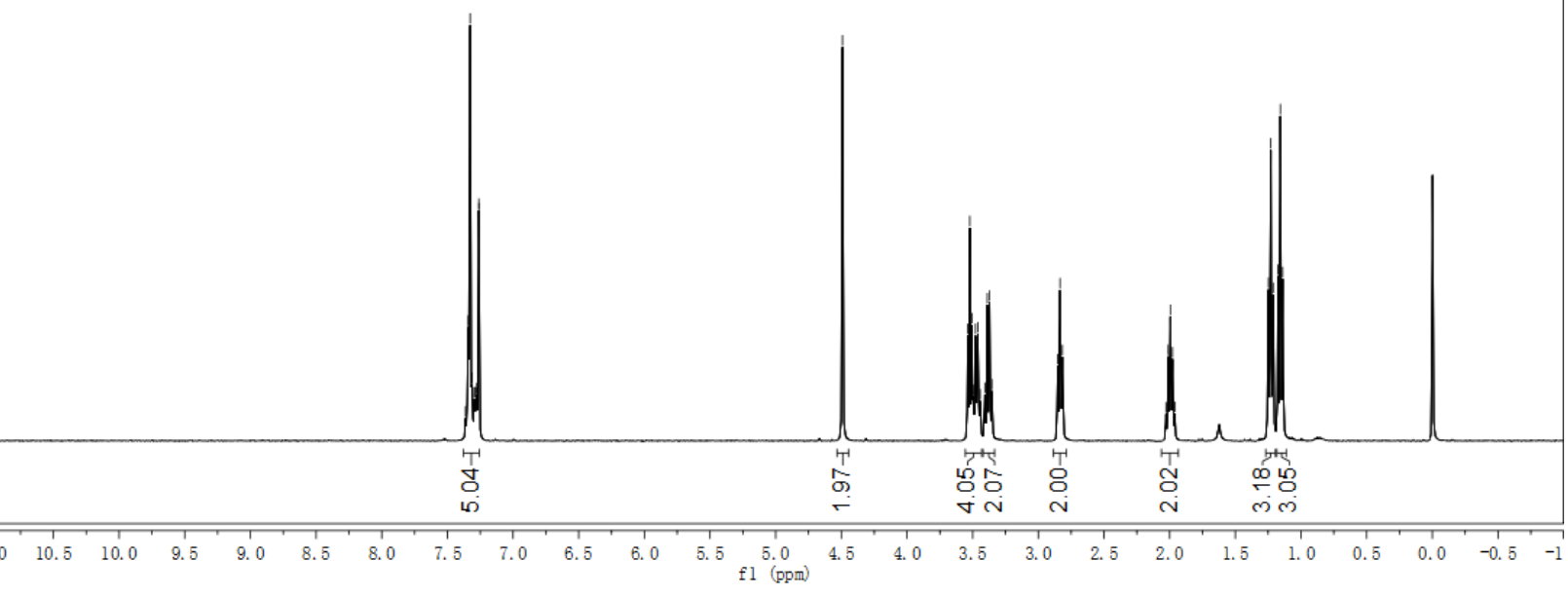



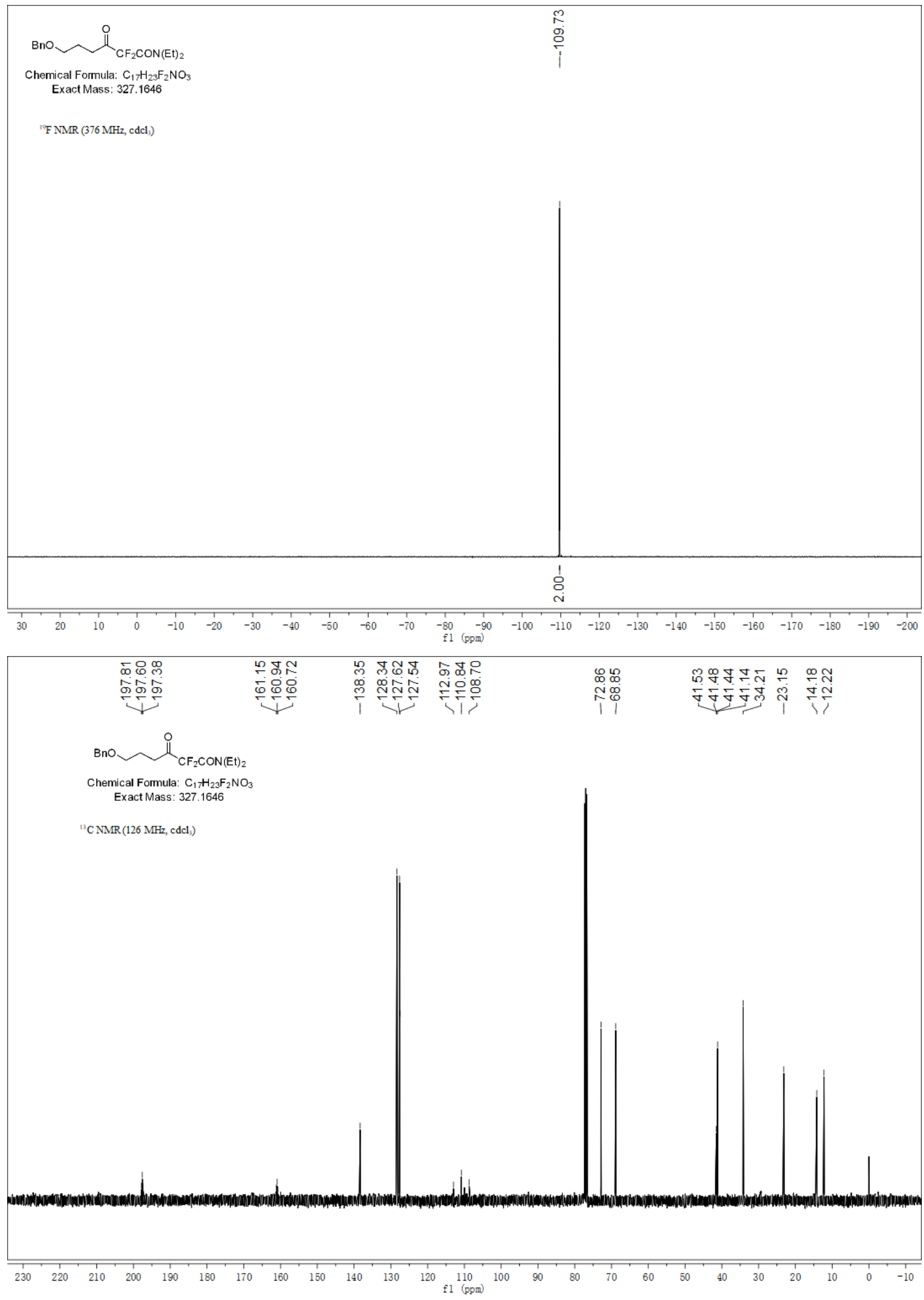
tert-Butyl-7-(diethylamino)-6,6-difluoro-5,7-dioxoheptanoate (7c).
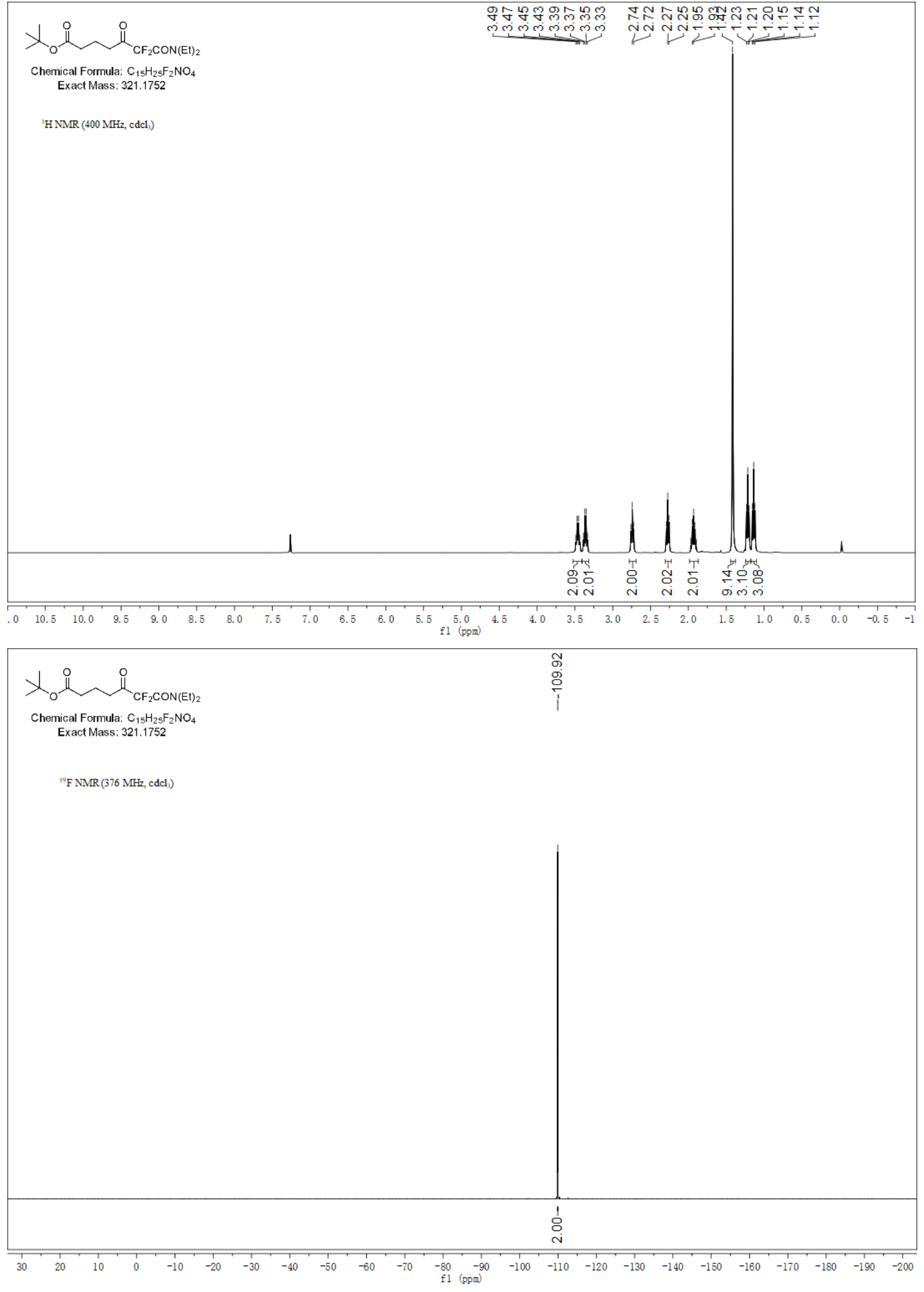


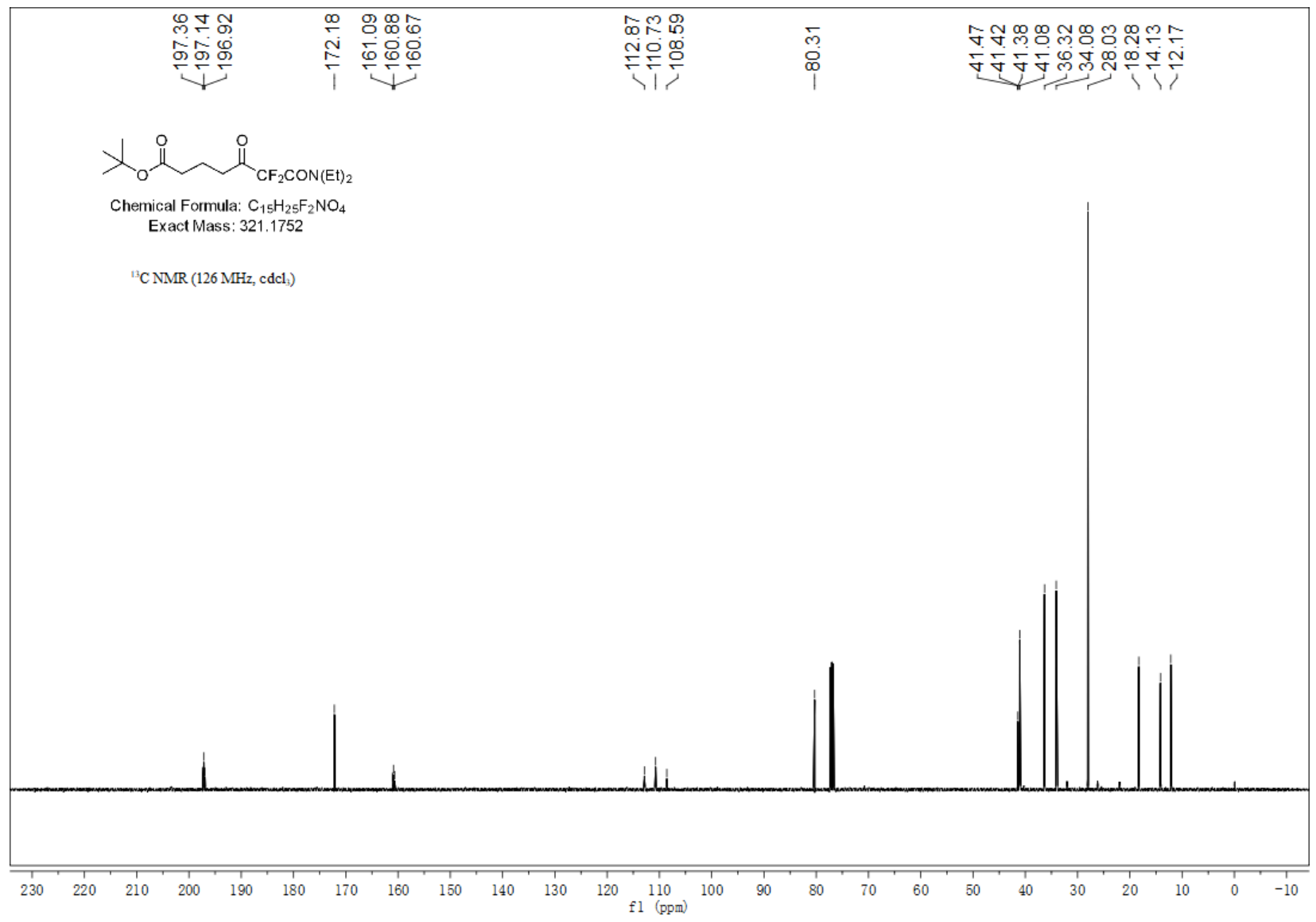

9-(Diethylamino)-8,8-difluoro-7,9-dioxononyl 2,4,6-trifluorobenzoate (7d).

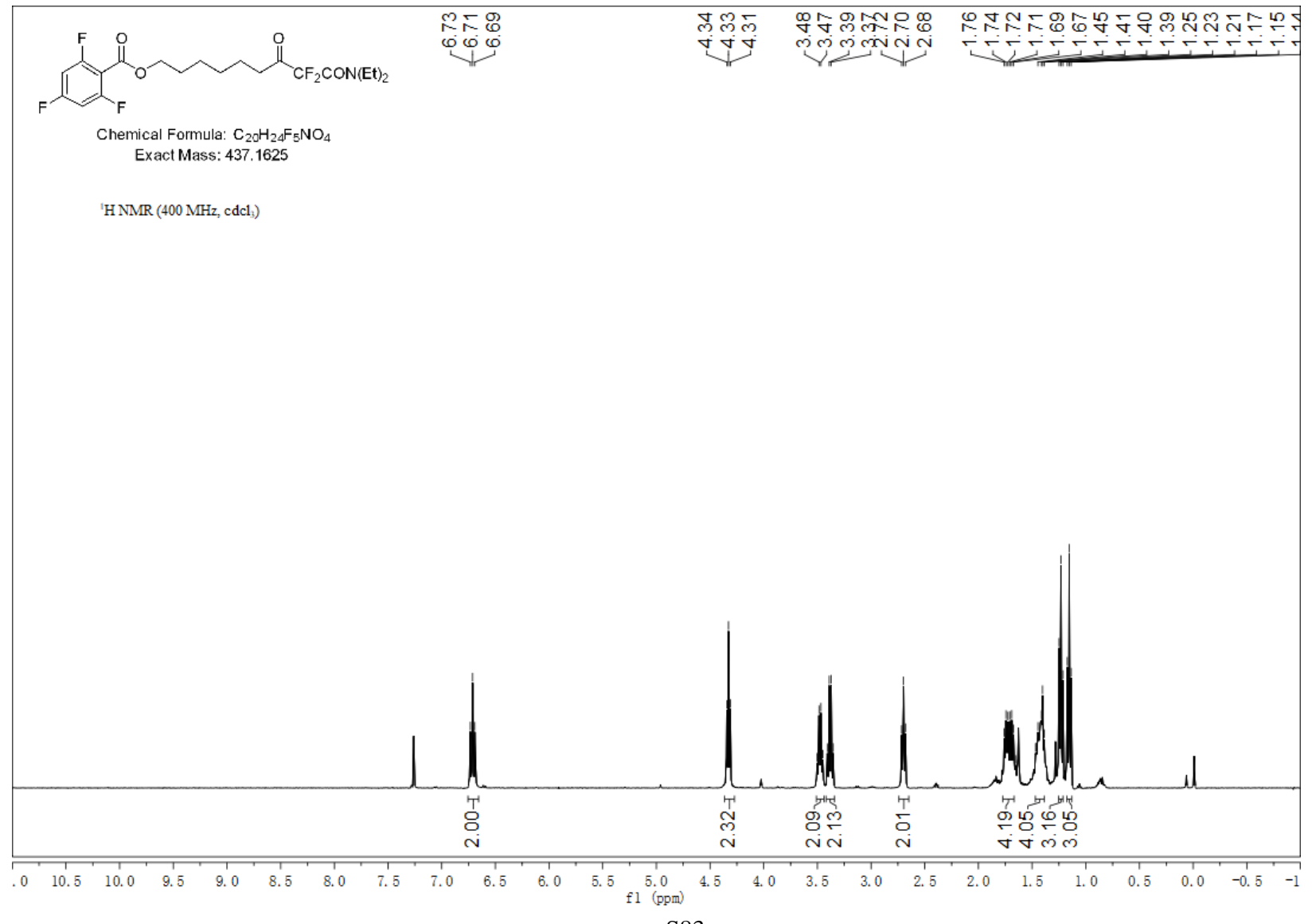



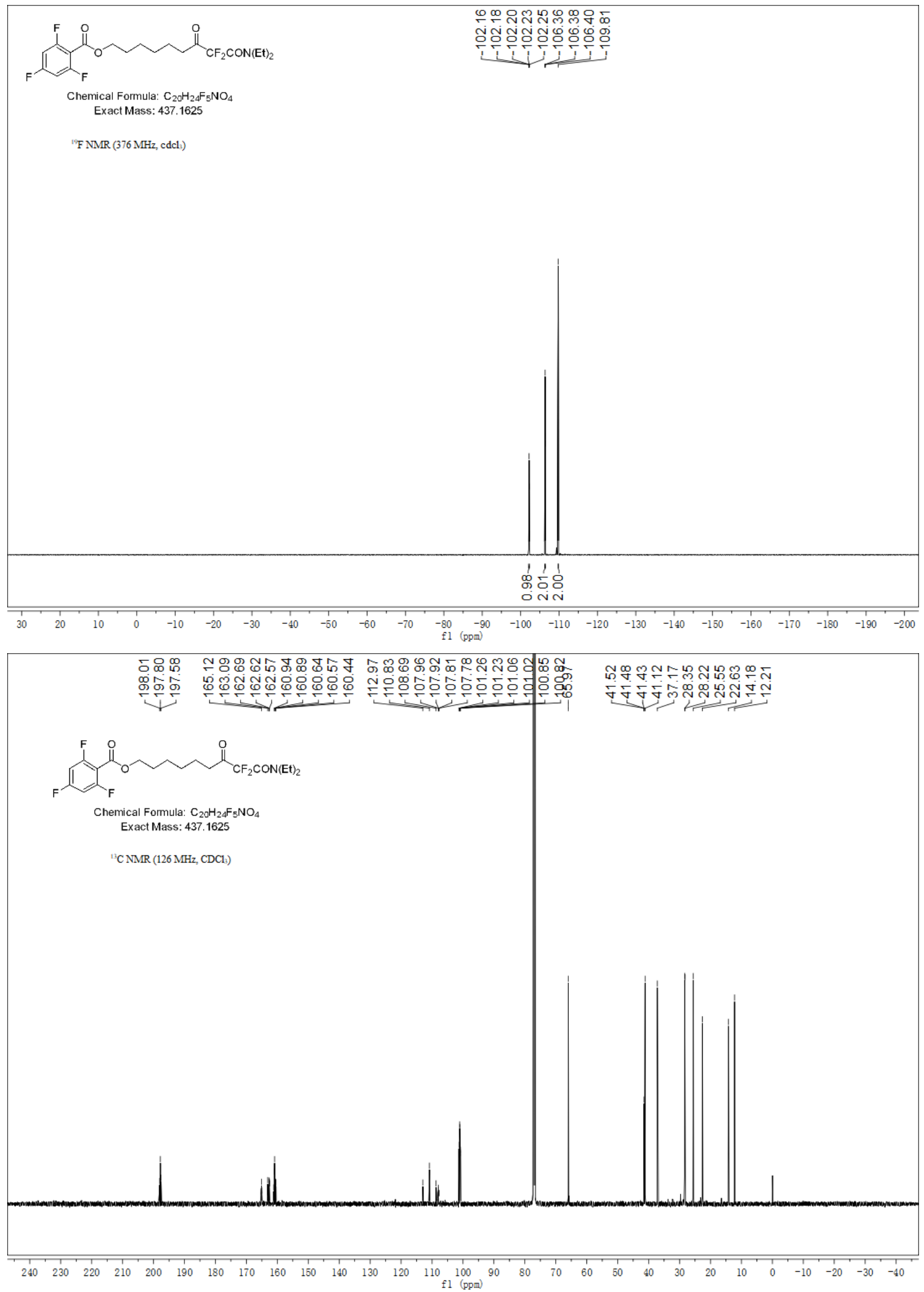
9-(Diethylamino)-8,8-difluoro-7,9-dioxononyl-4-methylthiazole-5-carboxylate (7e).
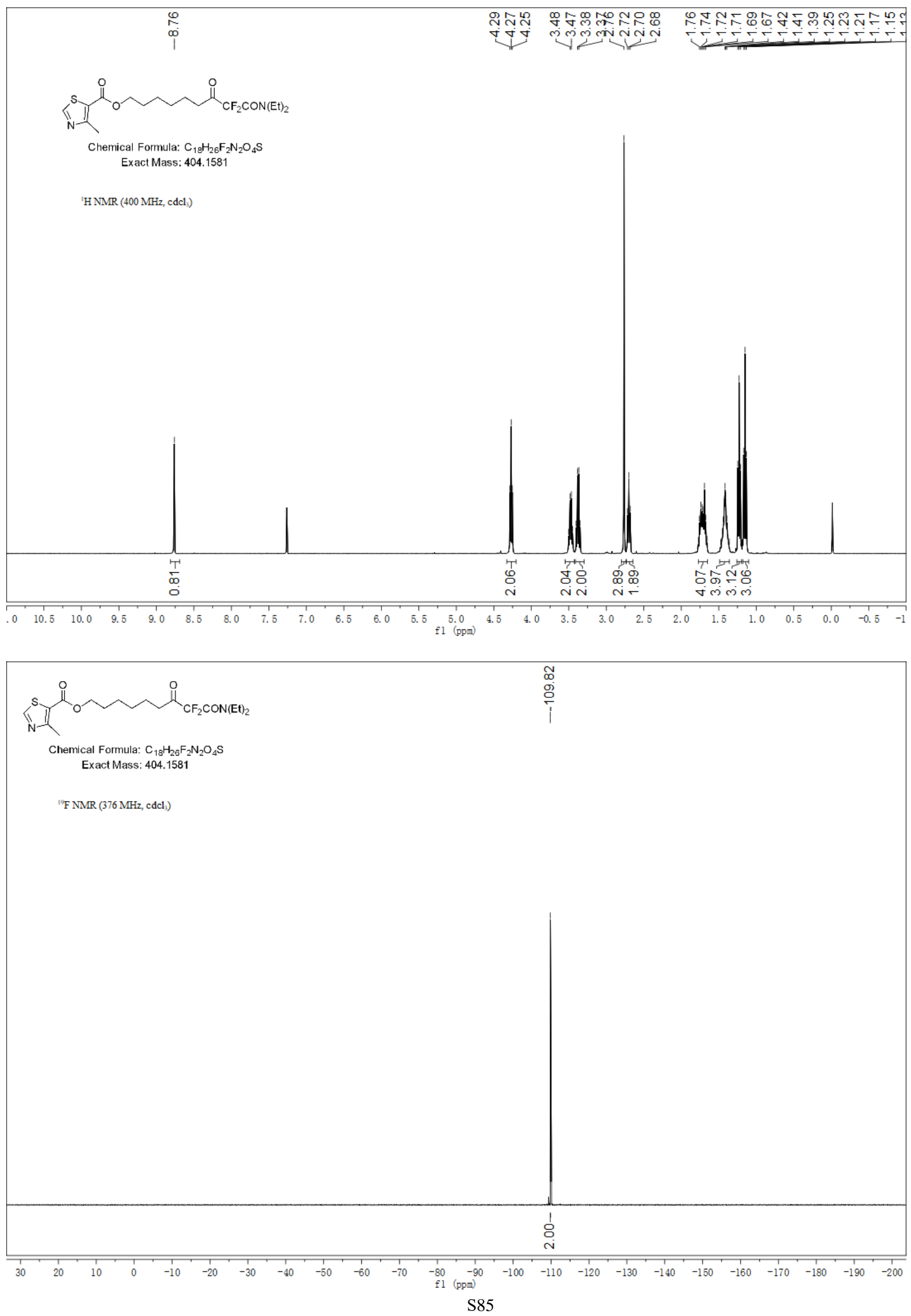


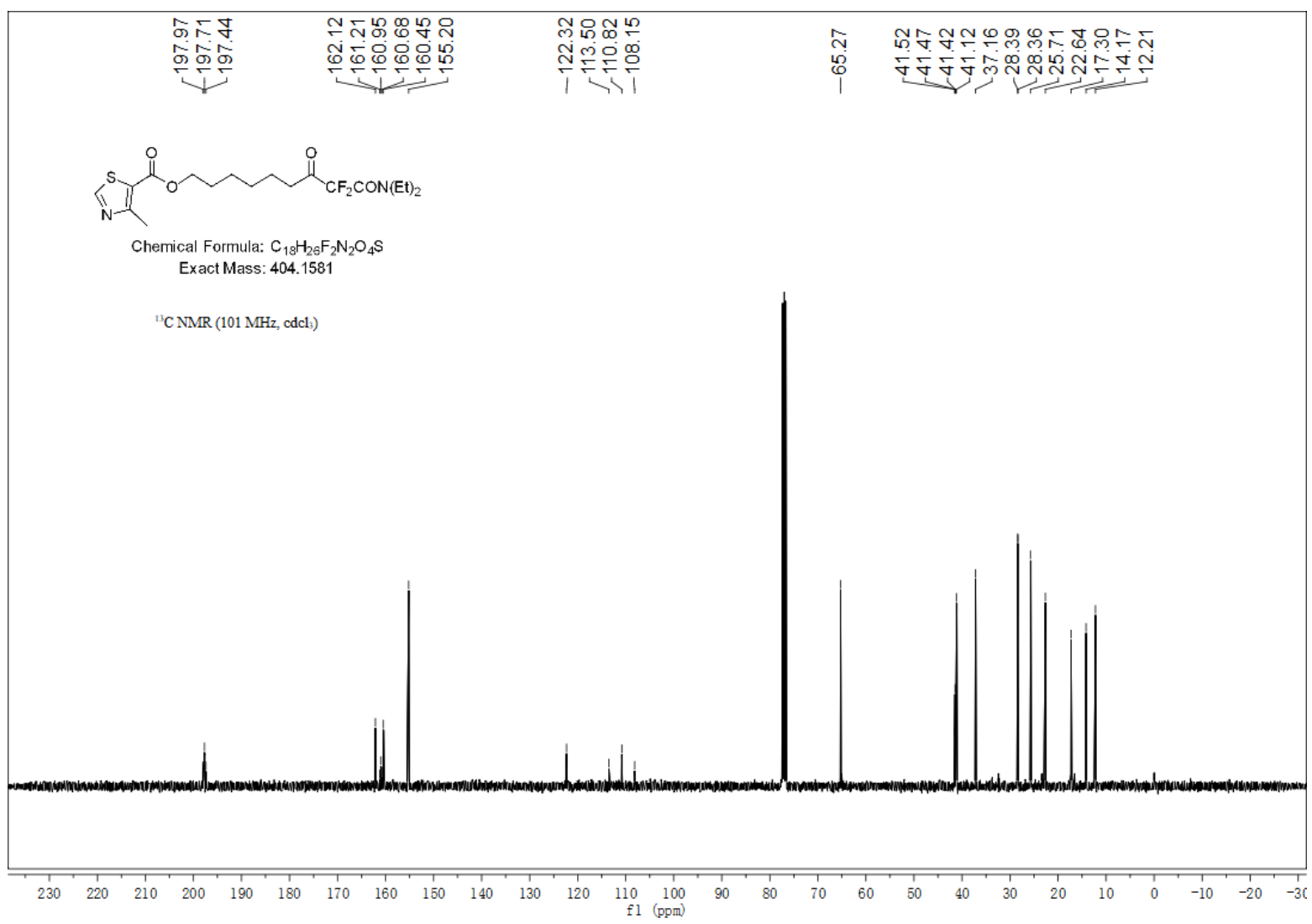

(S)-9-(Diethylamino)-8,8-difluoro-7,9-dioxononyl 2-((tert-butoxycarbonyl)amino)propanoate (7f).

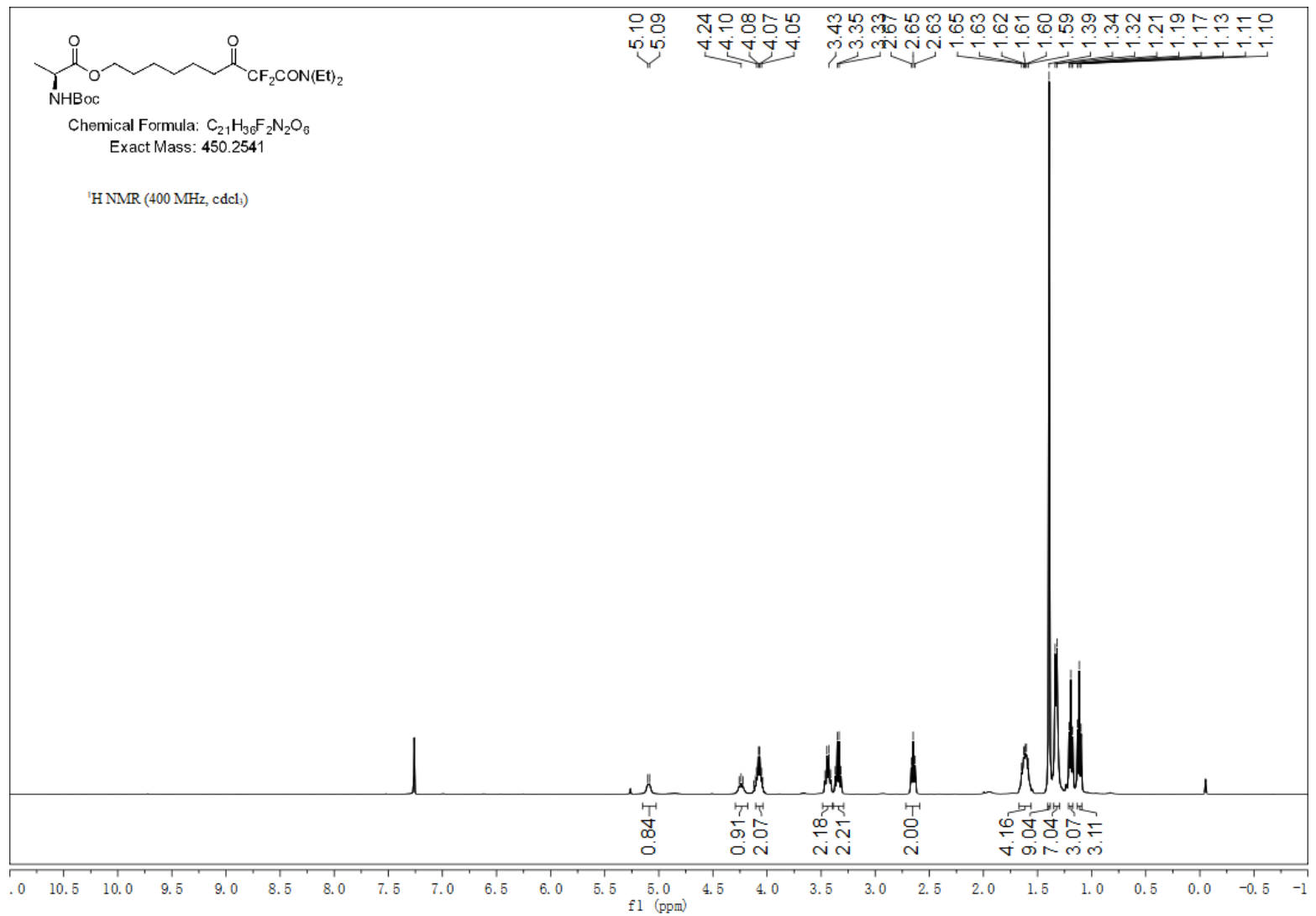



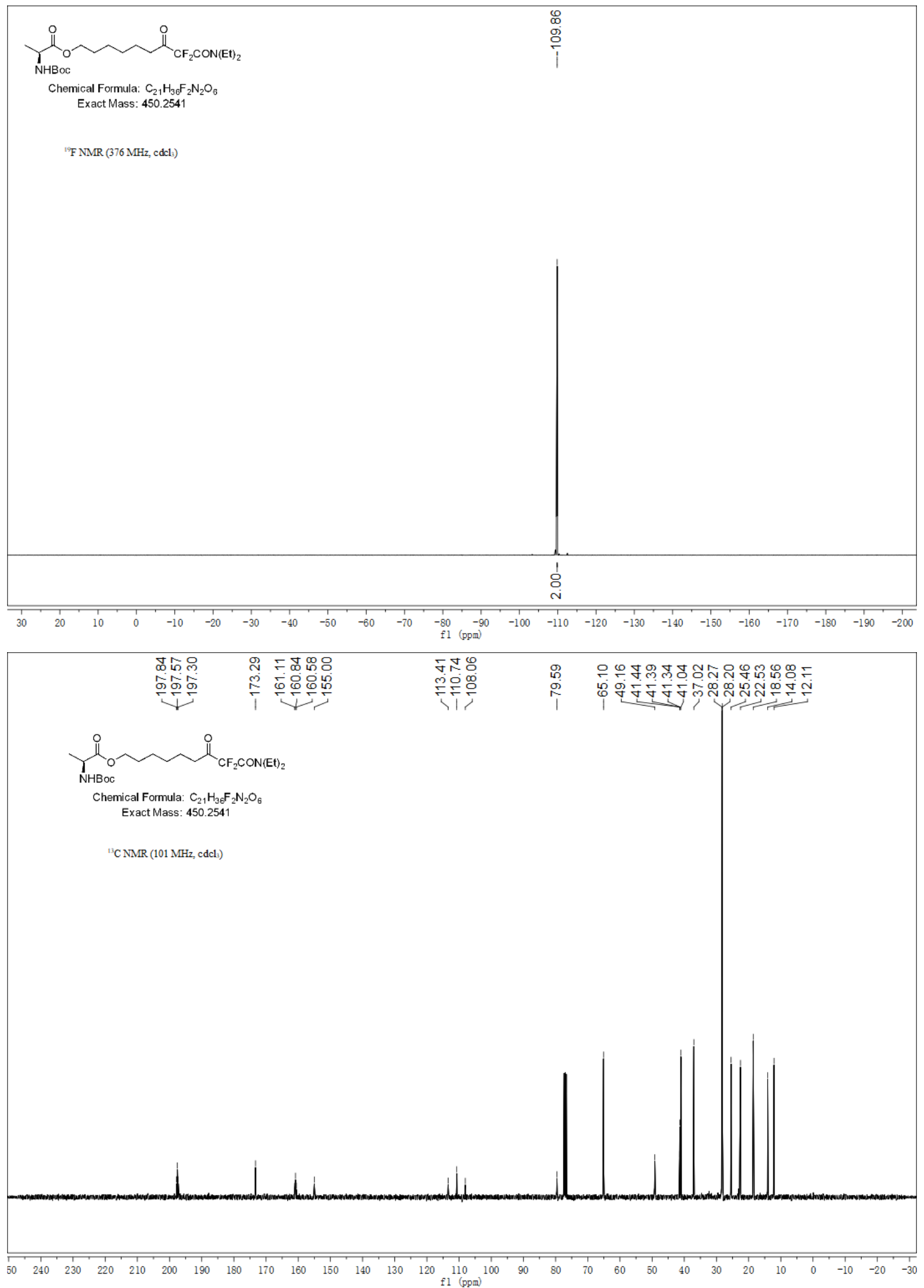
N-Butyl-2,2-difluoro-3-oxo-6-phenylhexanamide (7g).
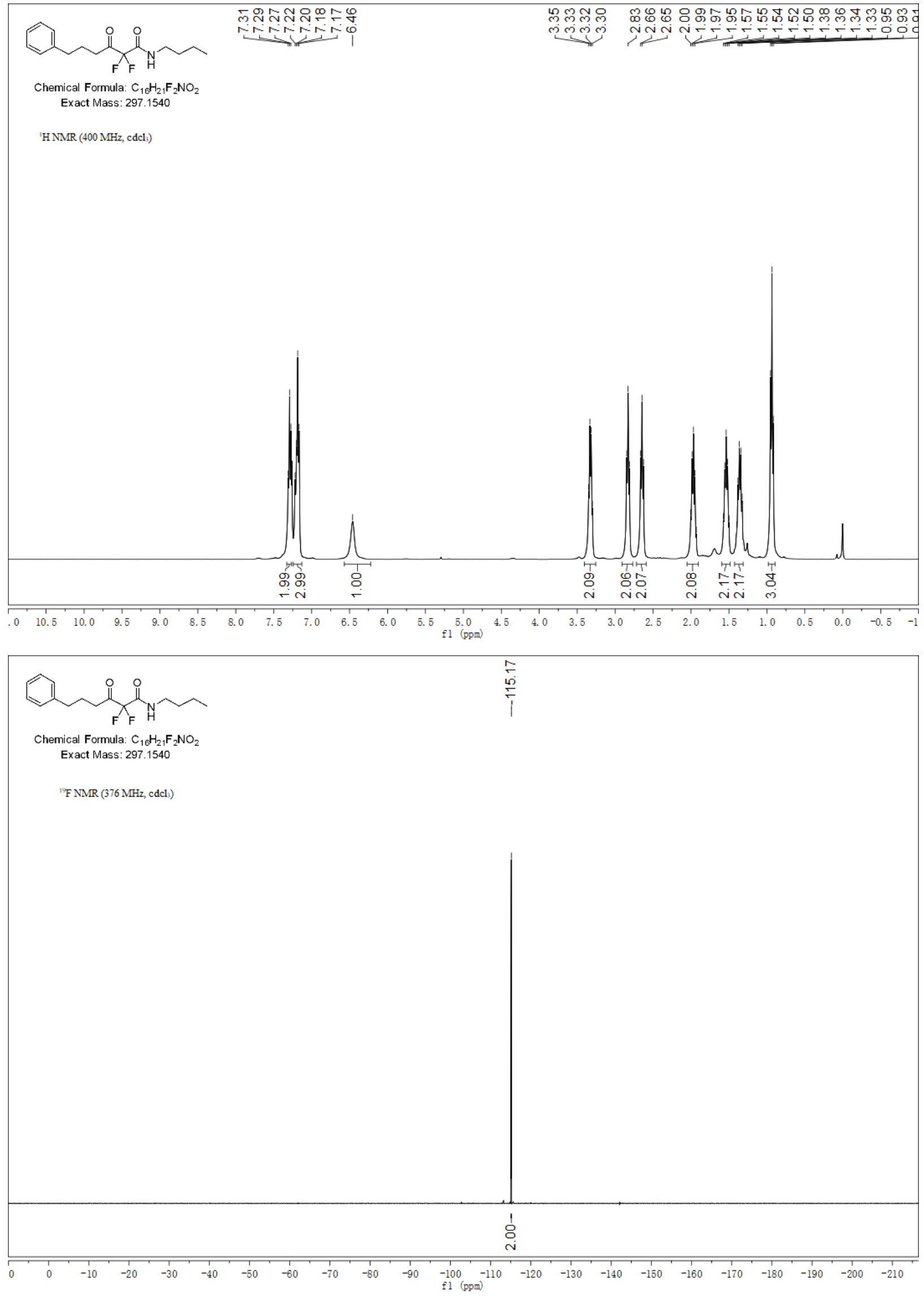


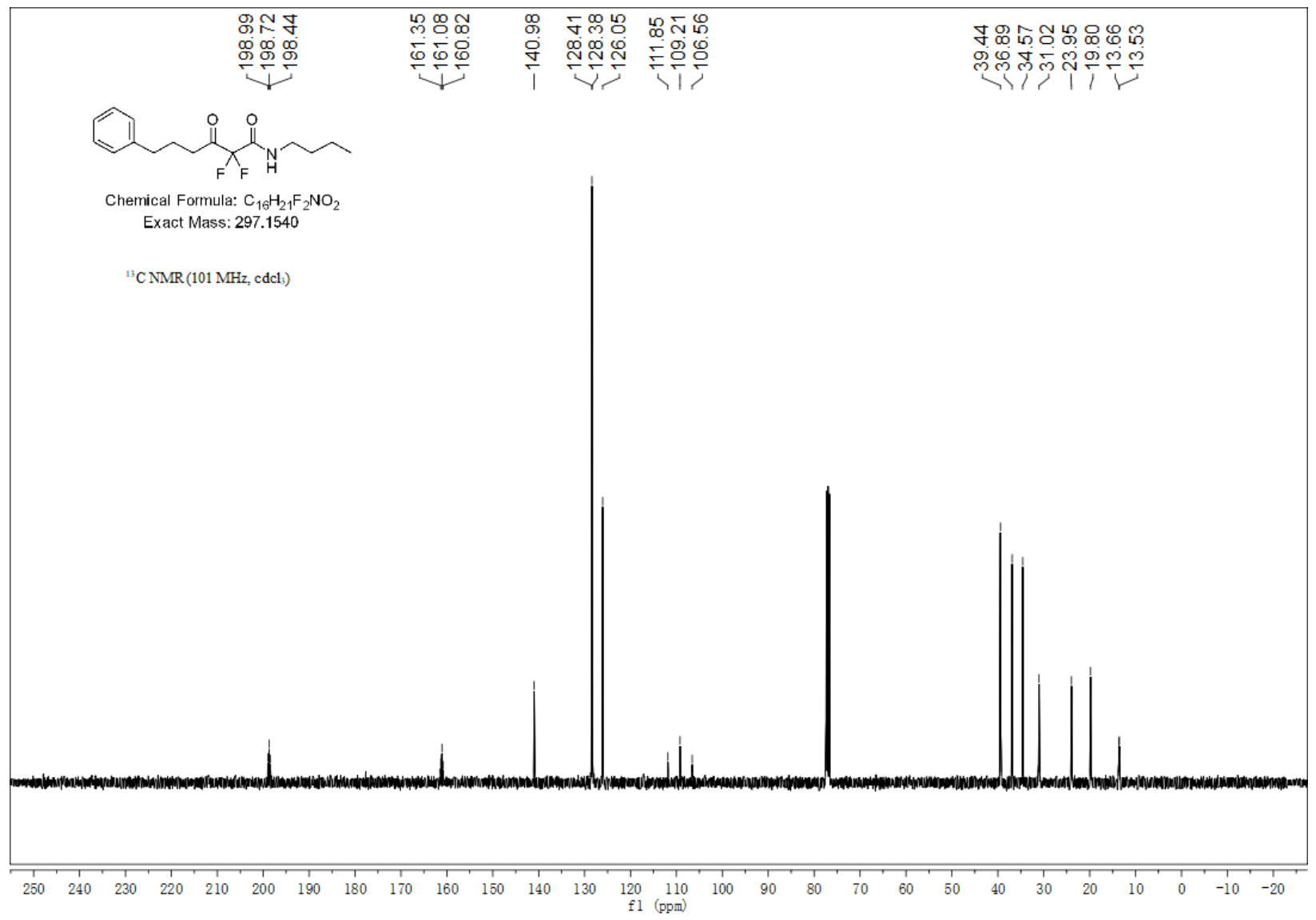

9-Ethoxy-8,8-difluoro-7,9-dioxononyl-2,4,6-trifluorobenzoate (7h).

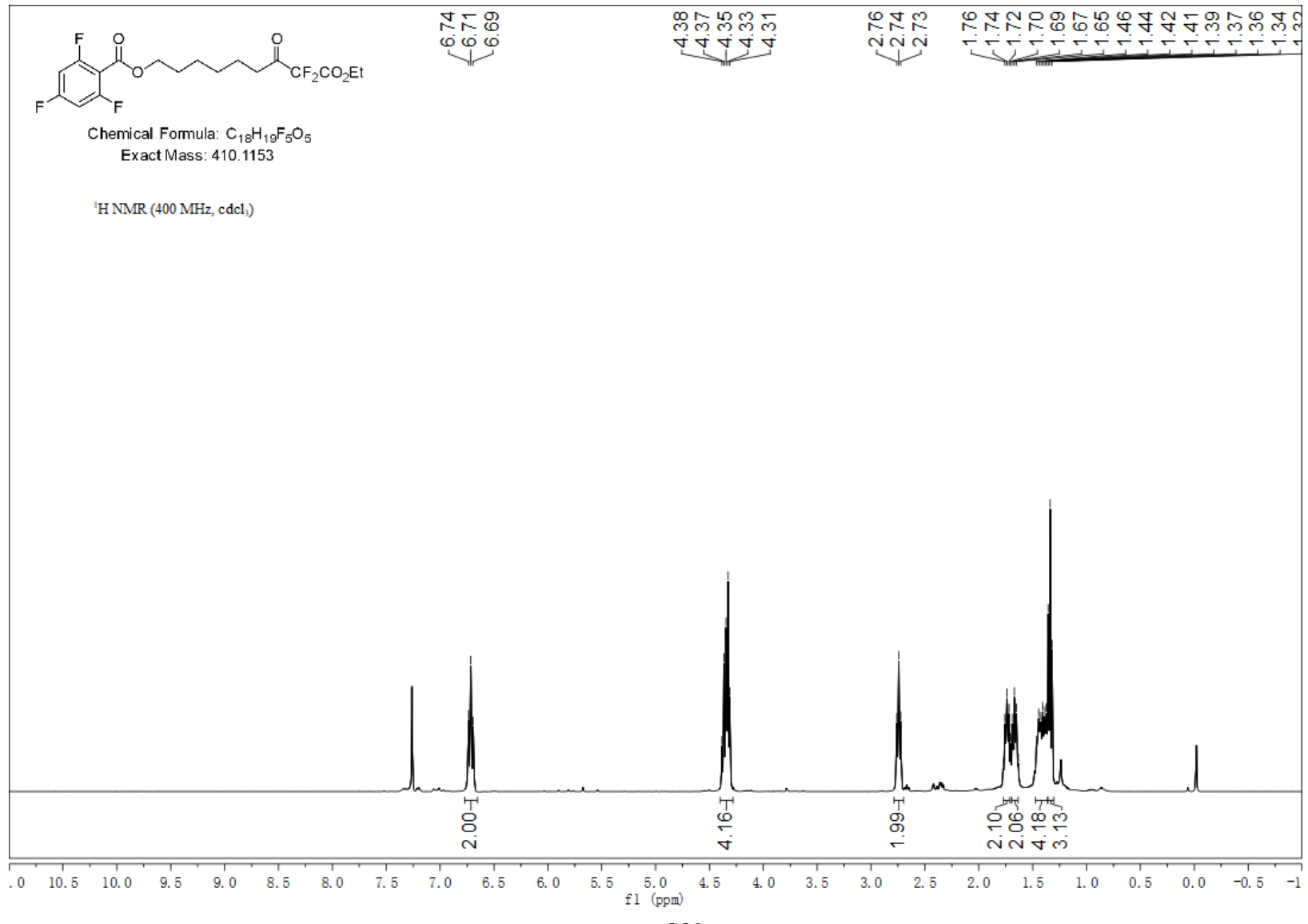



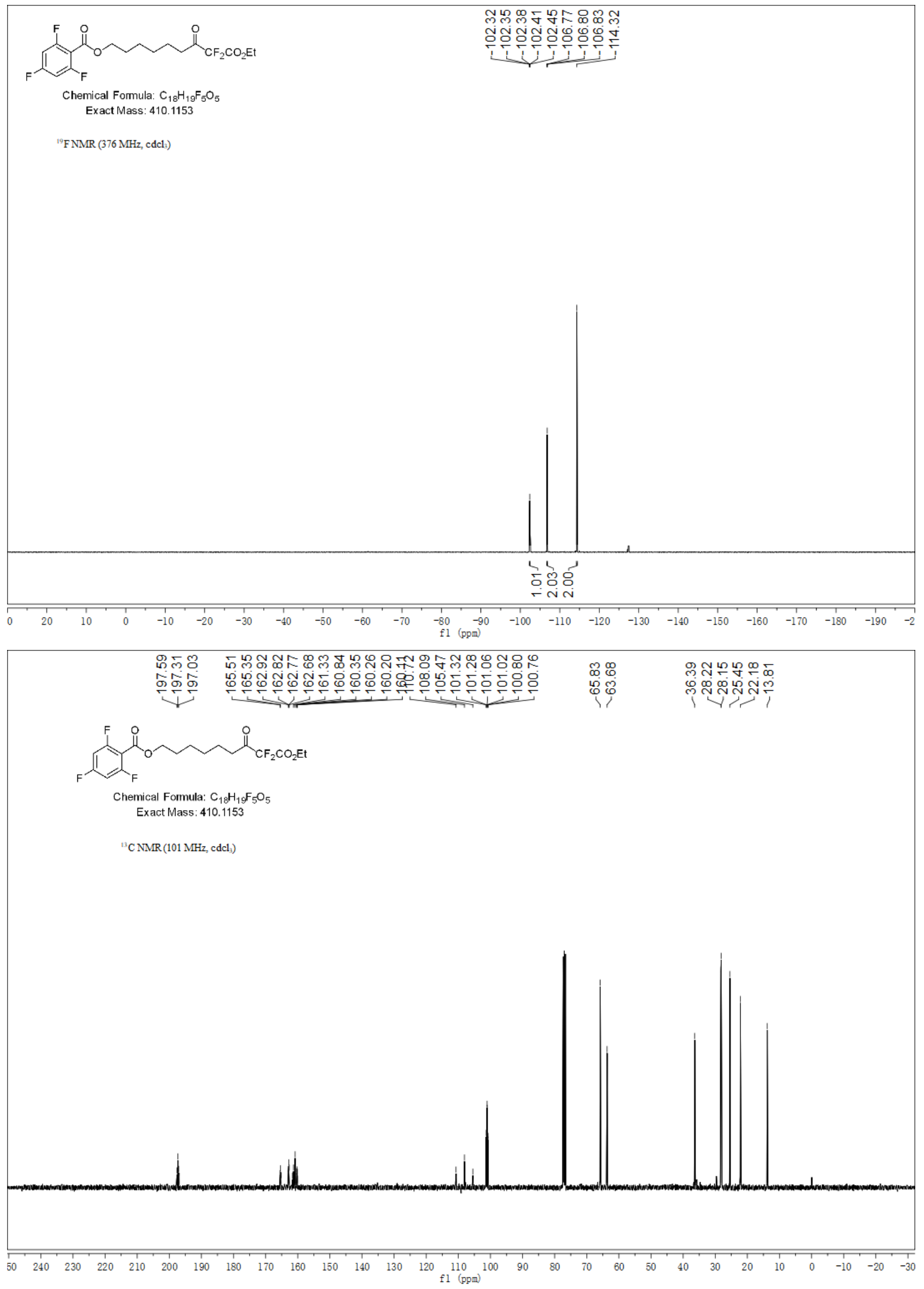
1,1-Difluoro-1-(5-methylbenzo[d] oxazol-2-yl)-5-phenylpentan-2-one (7i).
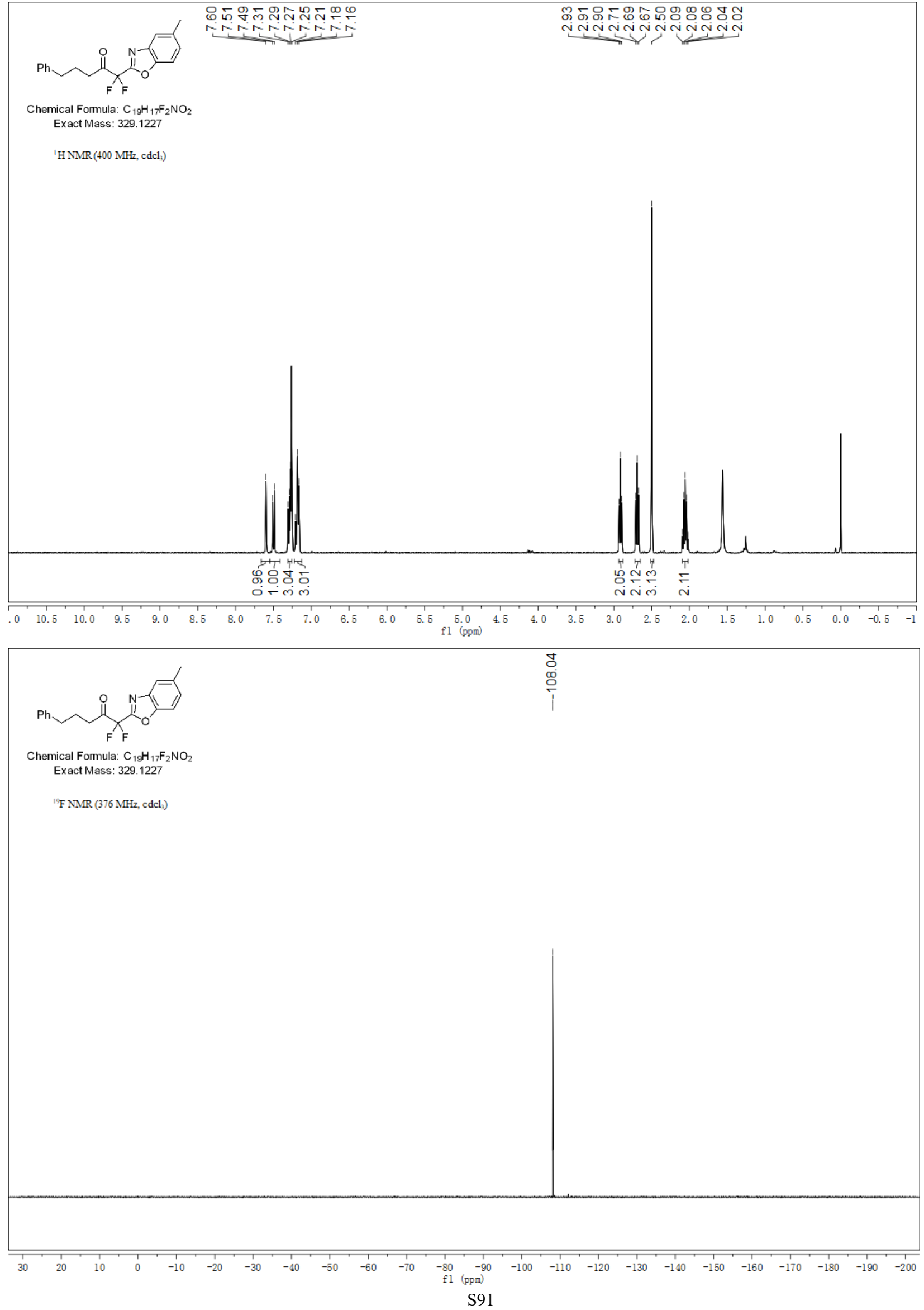


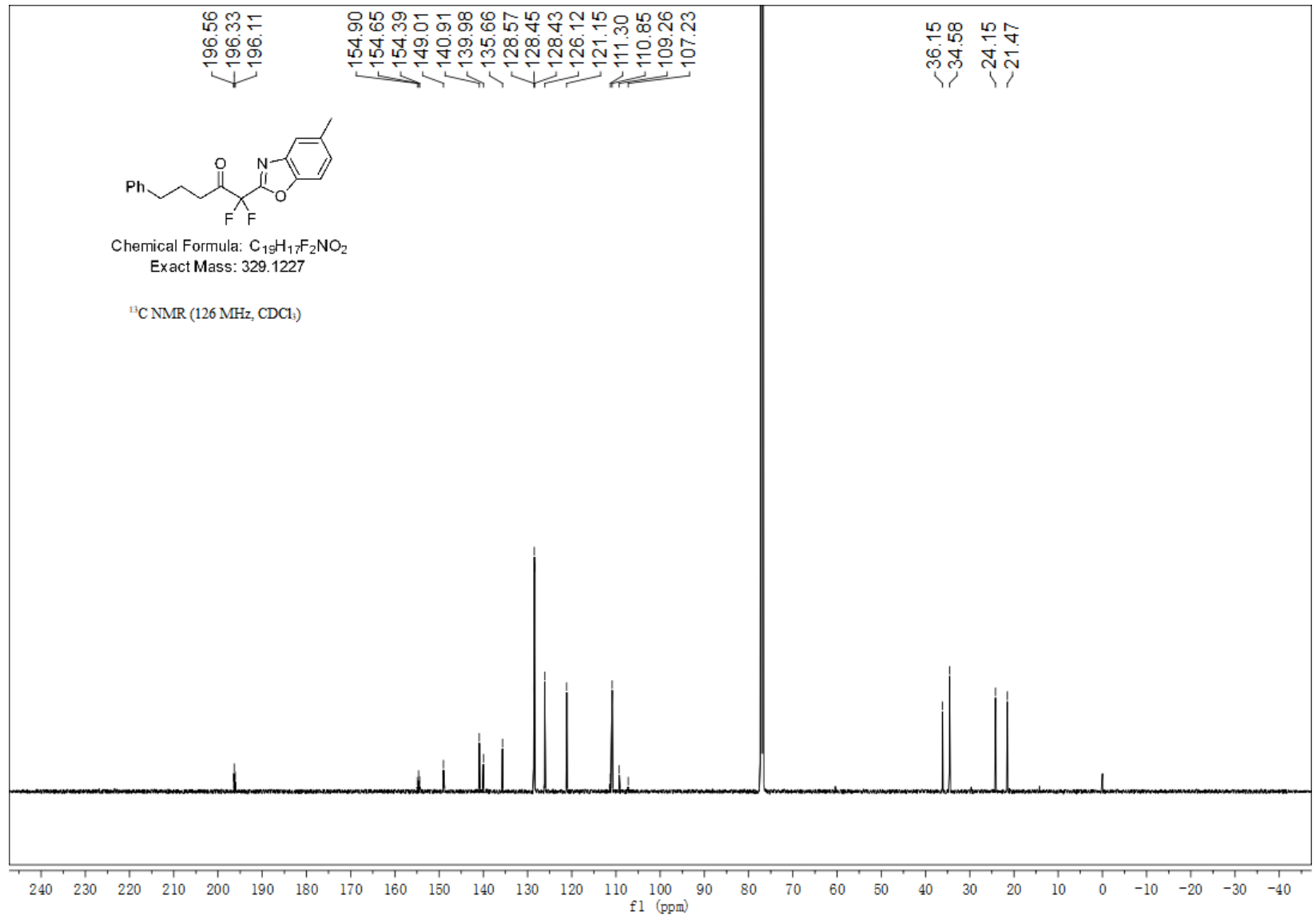

3,3-Difluoro-7-phenyl-1-(triisopropylsilyl)hept-1-yn-4-one (7j).

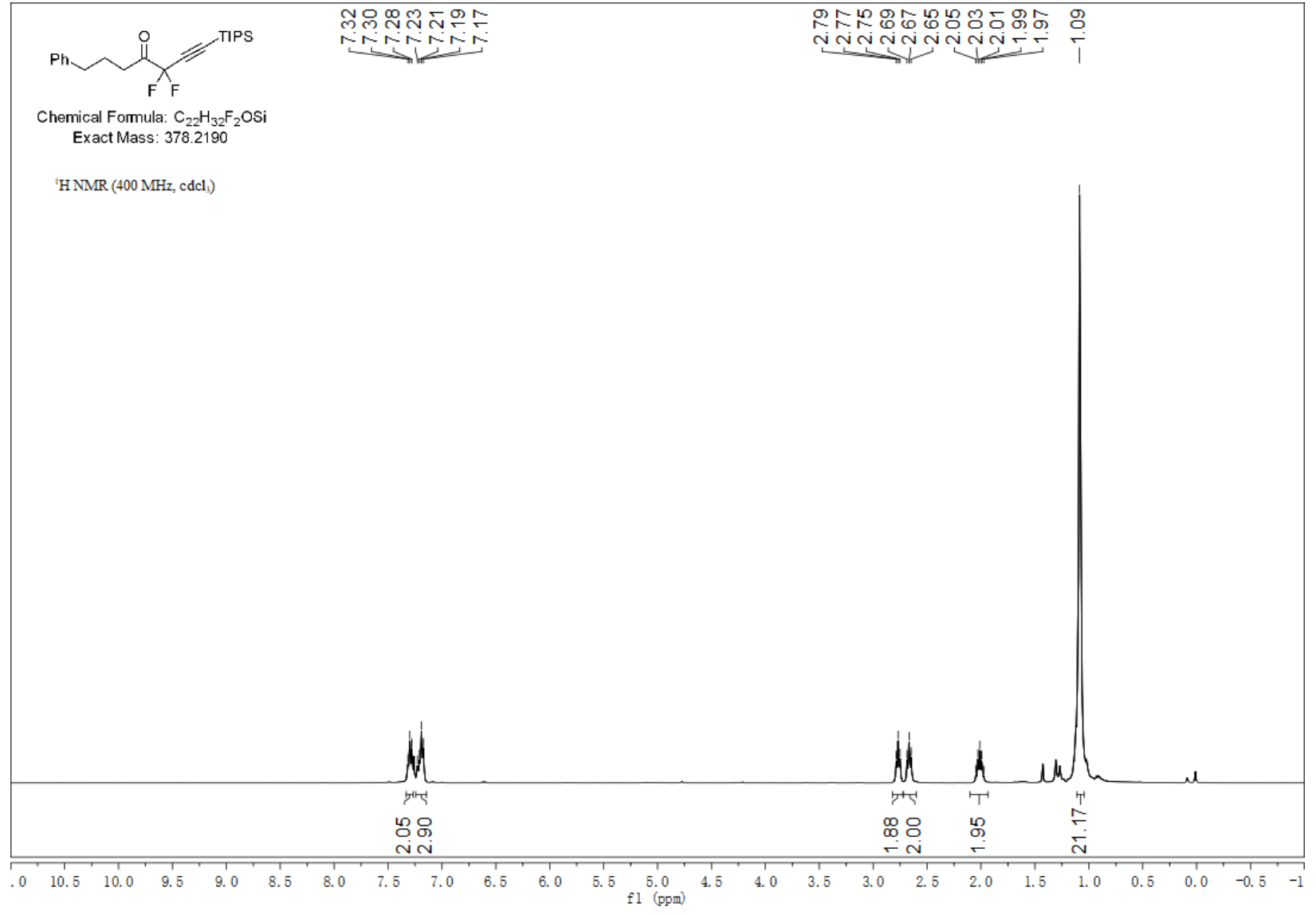



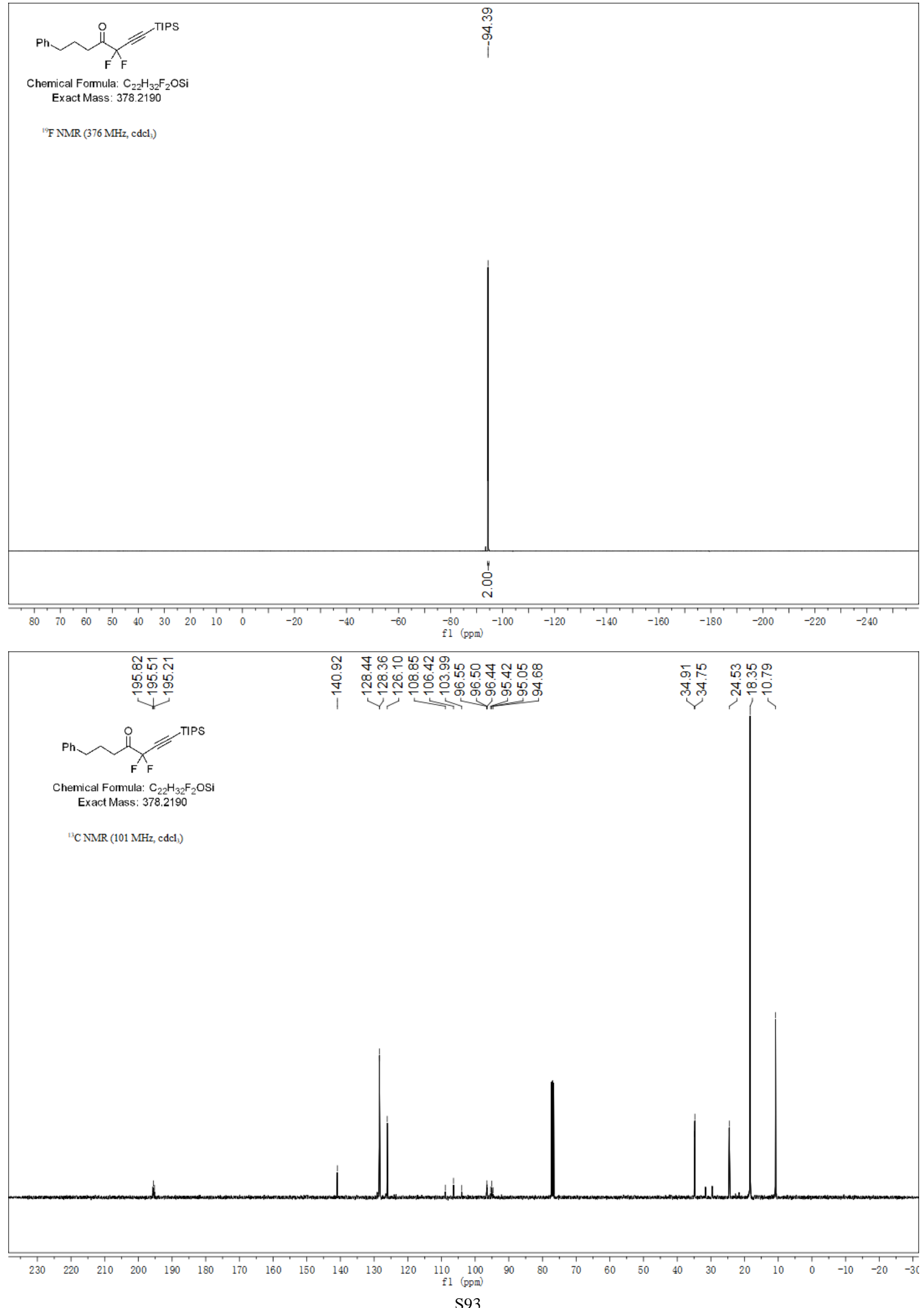
$(3 \mathrm{a} R, 4 R, 5 R, 7 R, 8 S, 9 R, 9 \mathrm{a} R, 12 R)-8$-Acetoxy-7-(4,4-difluoro-3-oxo-6-phenylhexyl)-4,7,9,12tetramethyl-3-oxodecahydro-4,9a-propanocyclopenta[8]annulen-5-yl 2-acetoxyacetate (9).
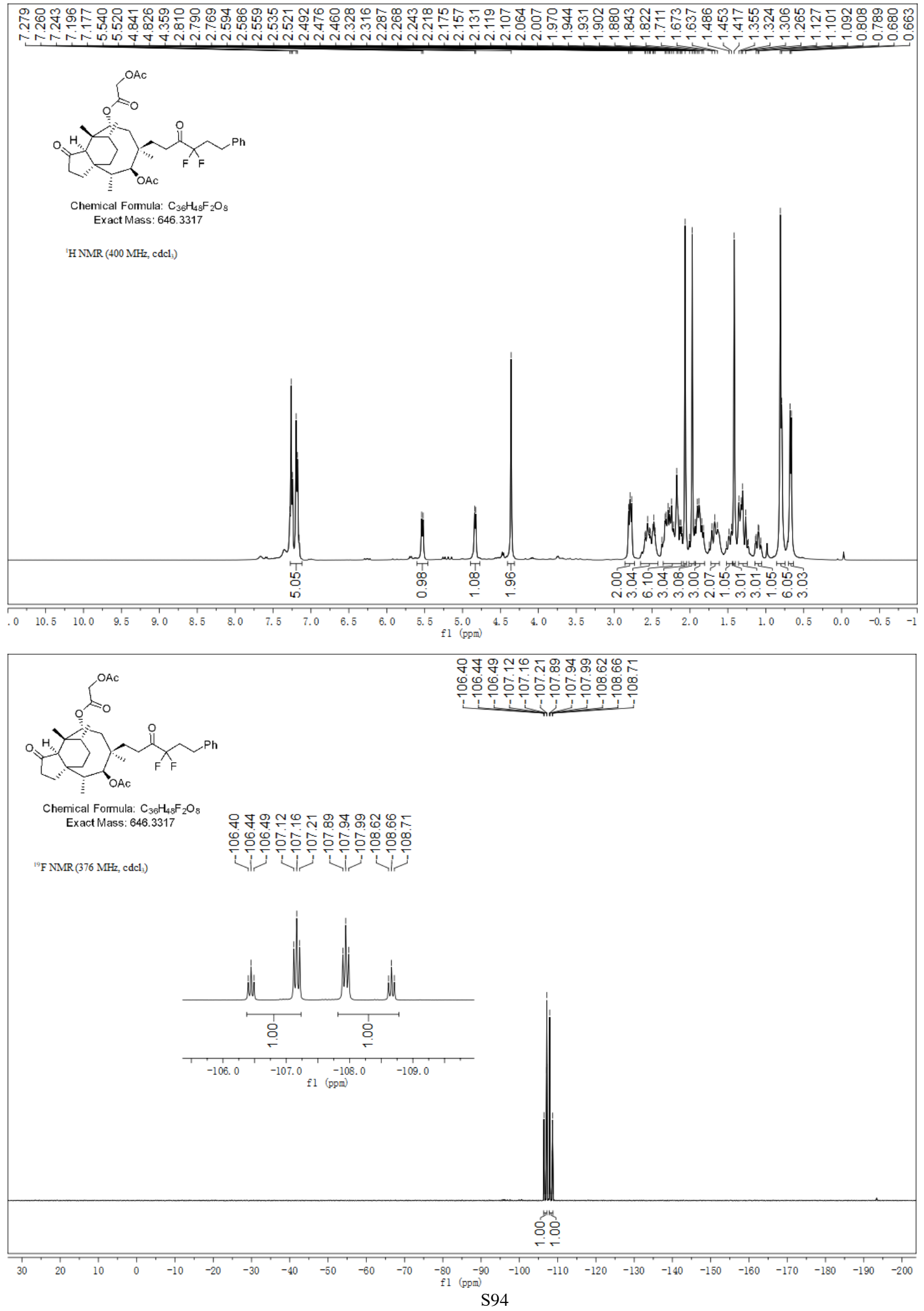


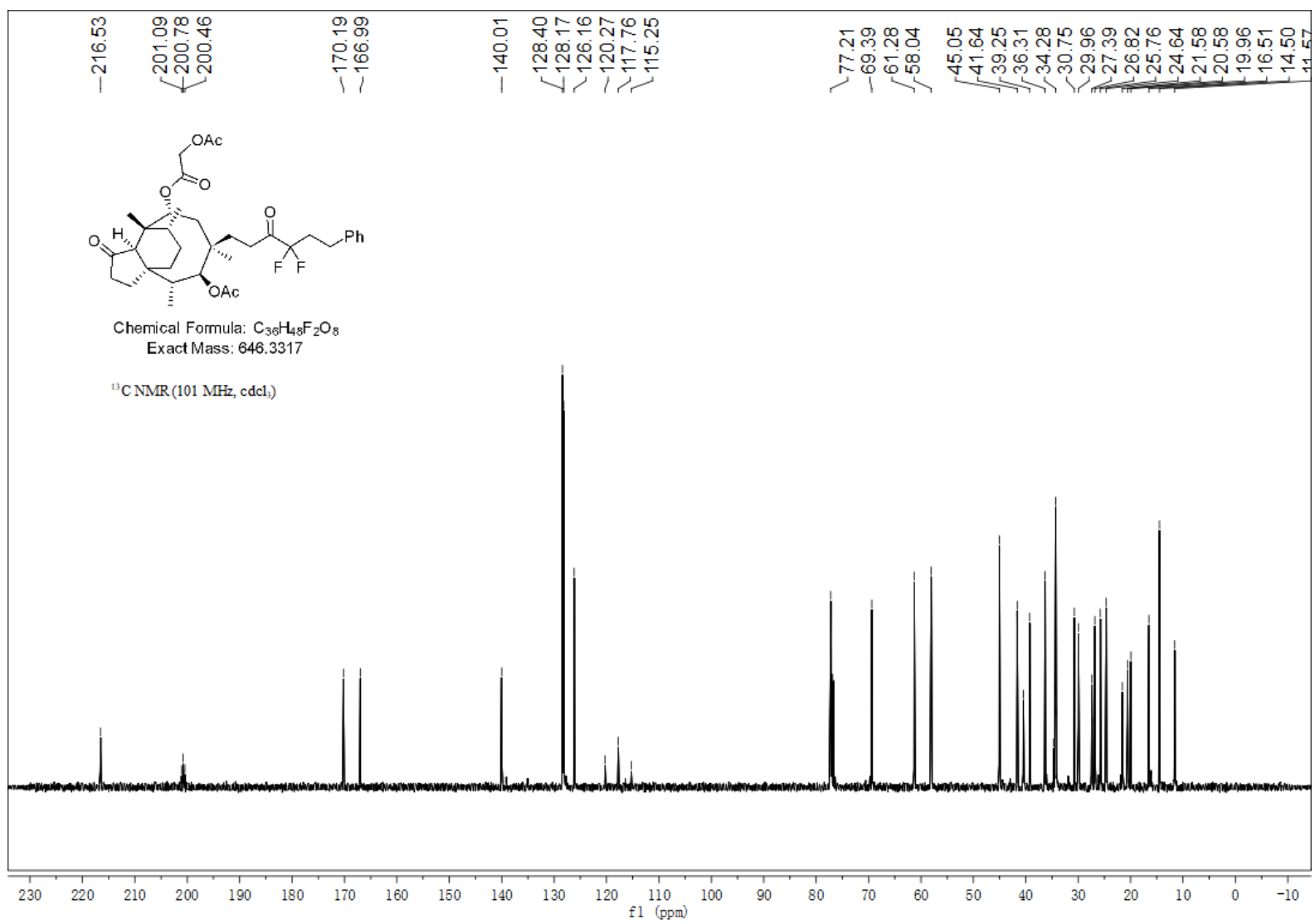

(3a $R, 4 R, 5 R, 6 a S)-4-(4,4-D i f l u o r o-3-0 x o-6-p h e n y l h e x y l)-2-o x o h e x a h y d r o-2 H-$ cyclopenta[$[b]$ furan-5-yl benzoate (11).

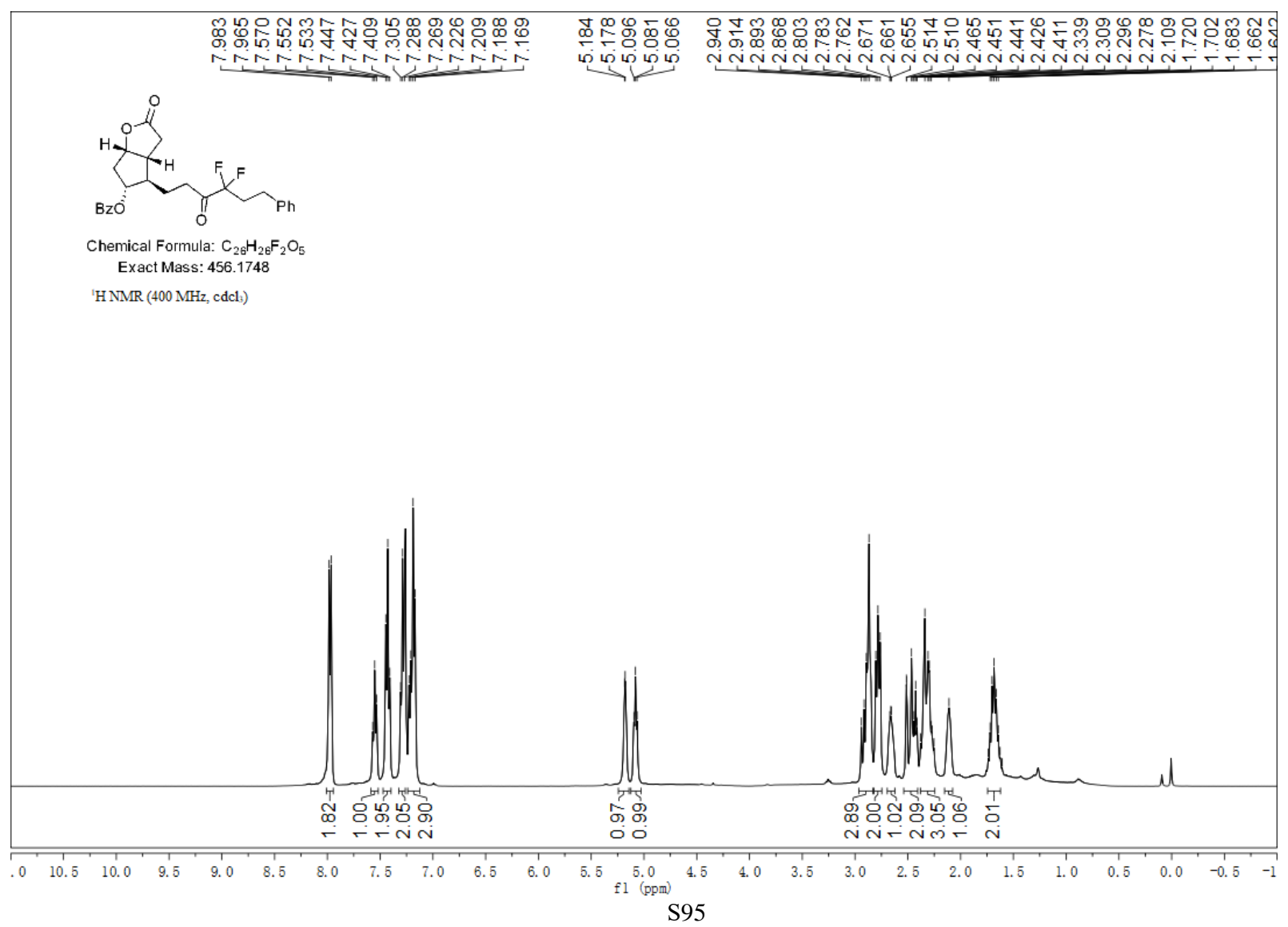



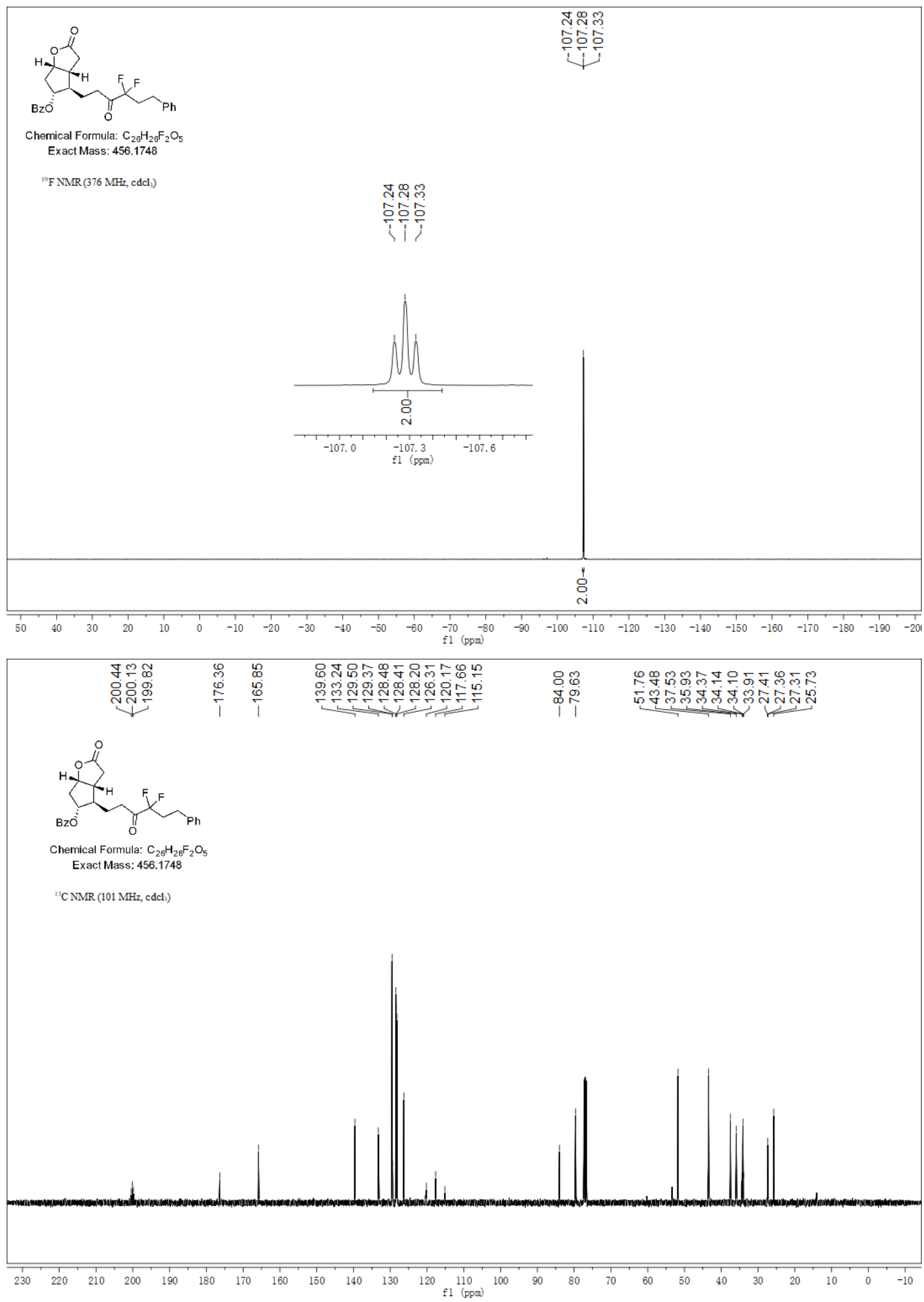
3,3-Difluoro-1,7-diphenylheptan-4-ol (12).
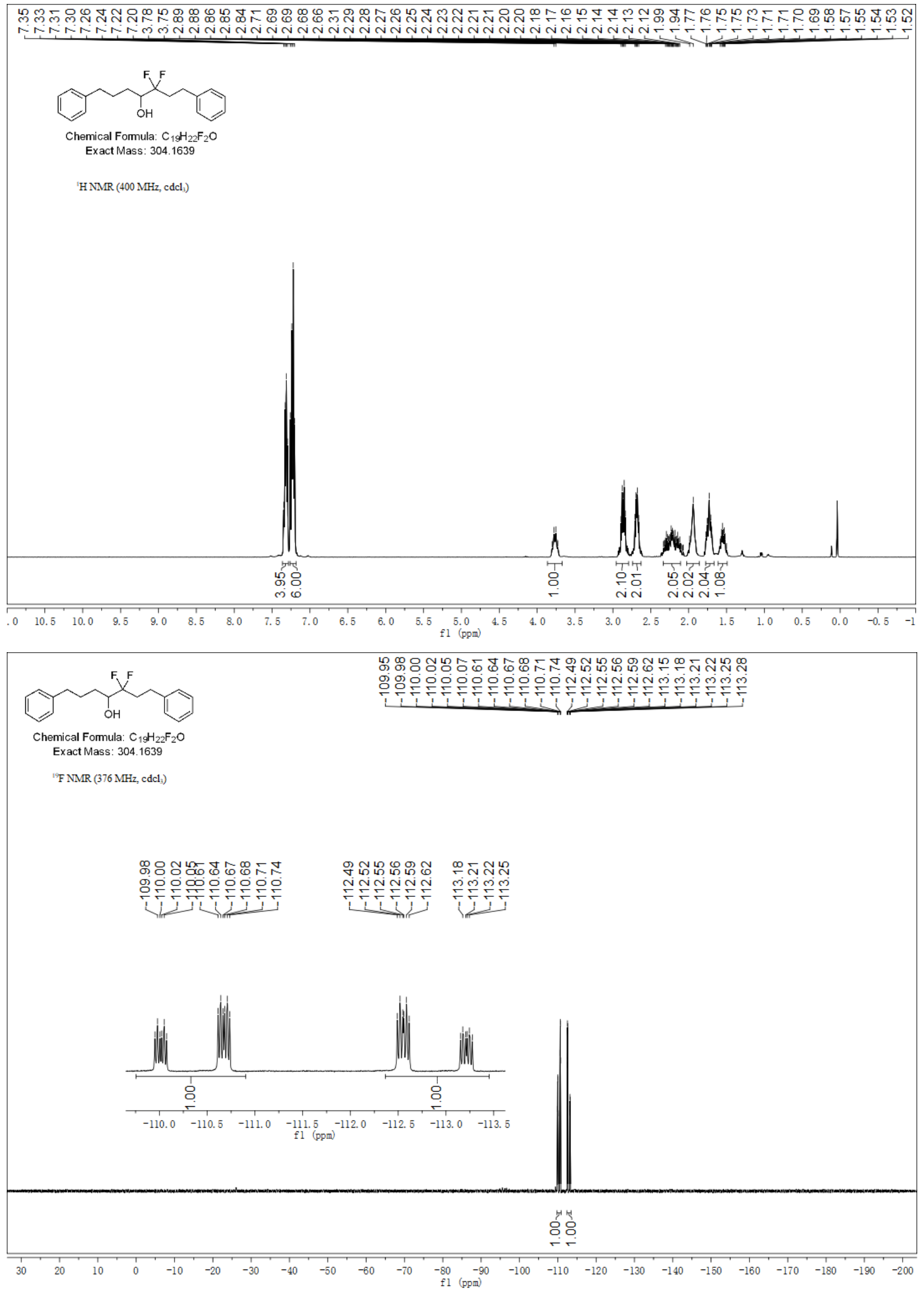


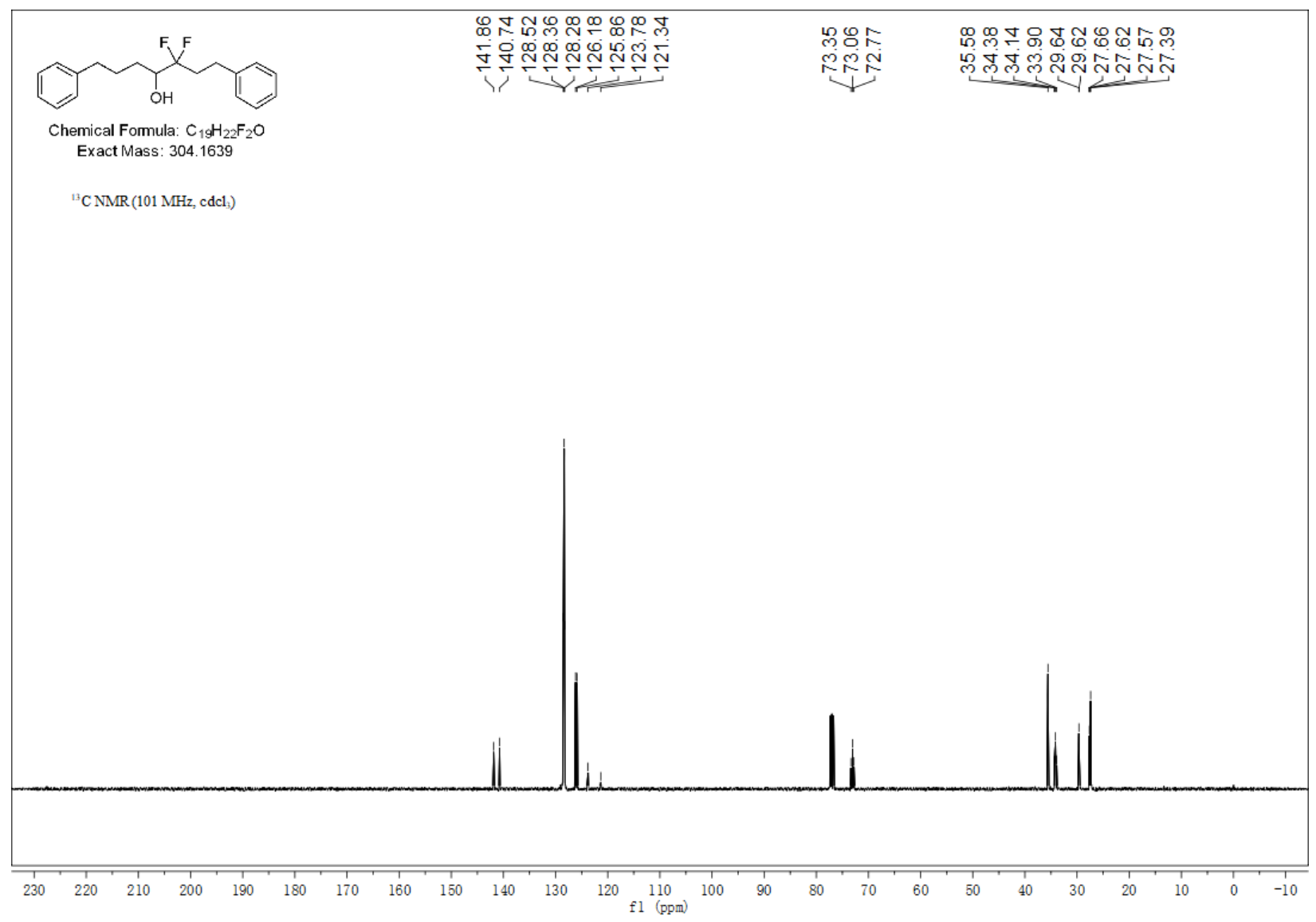

(3,3-Difluoro-4-methyleneheptane-1,7-diyl)dibenzene (13).

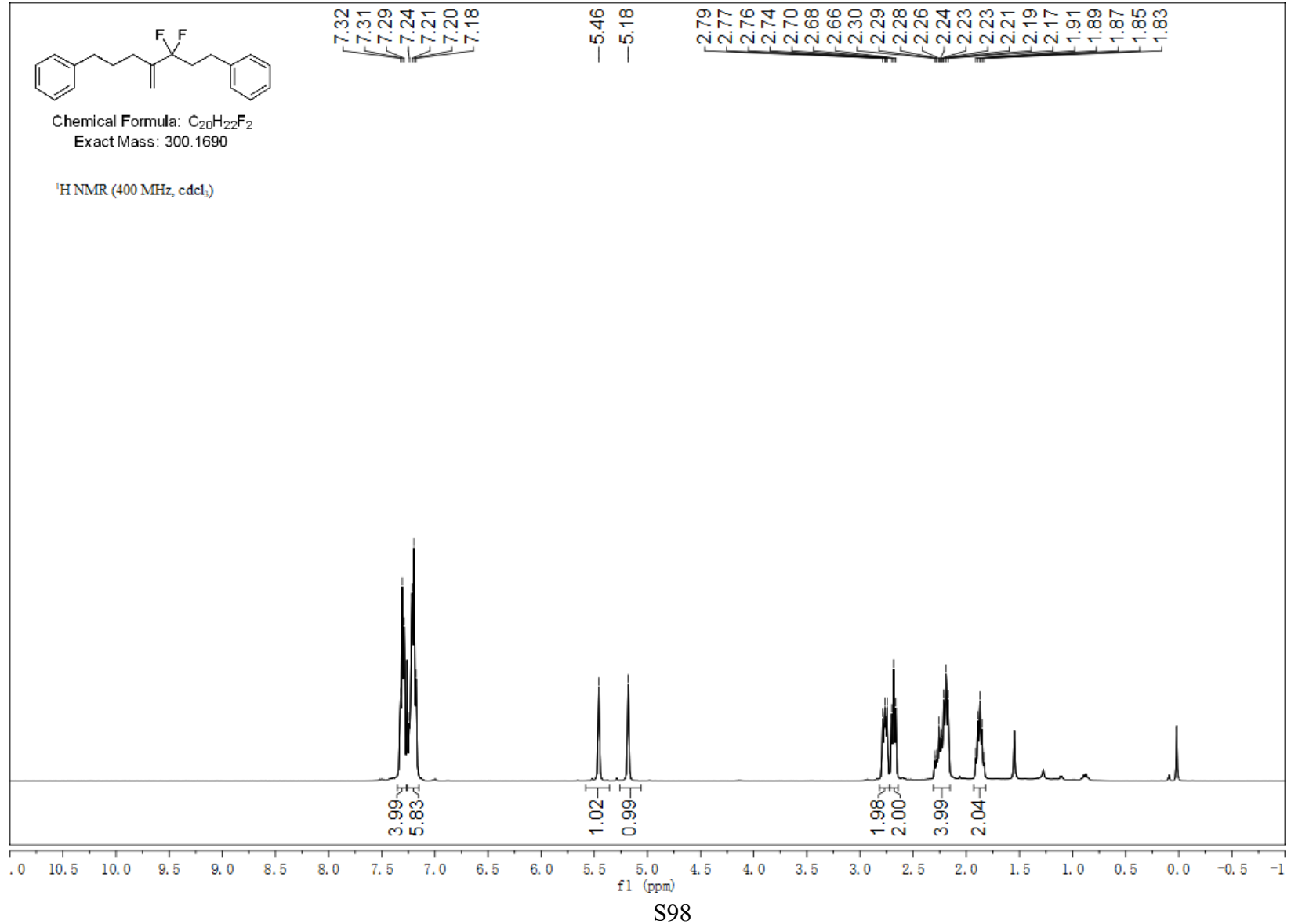



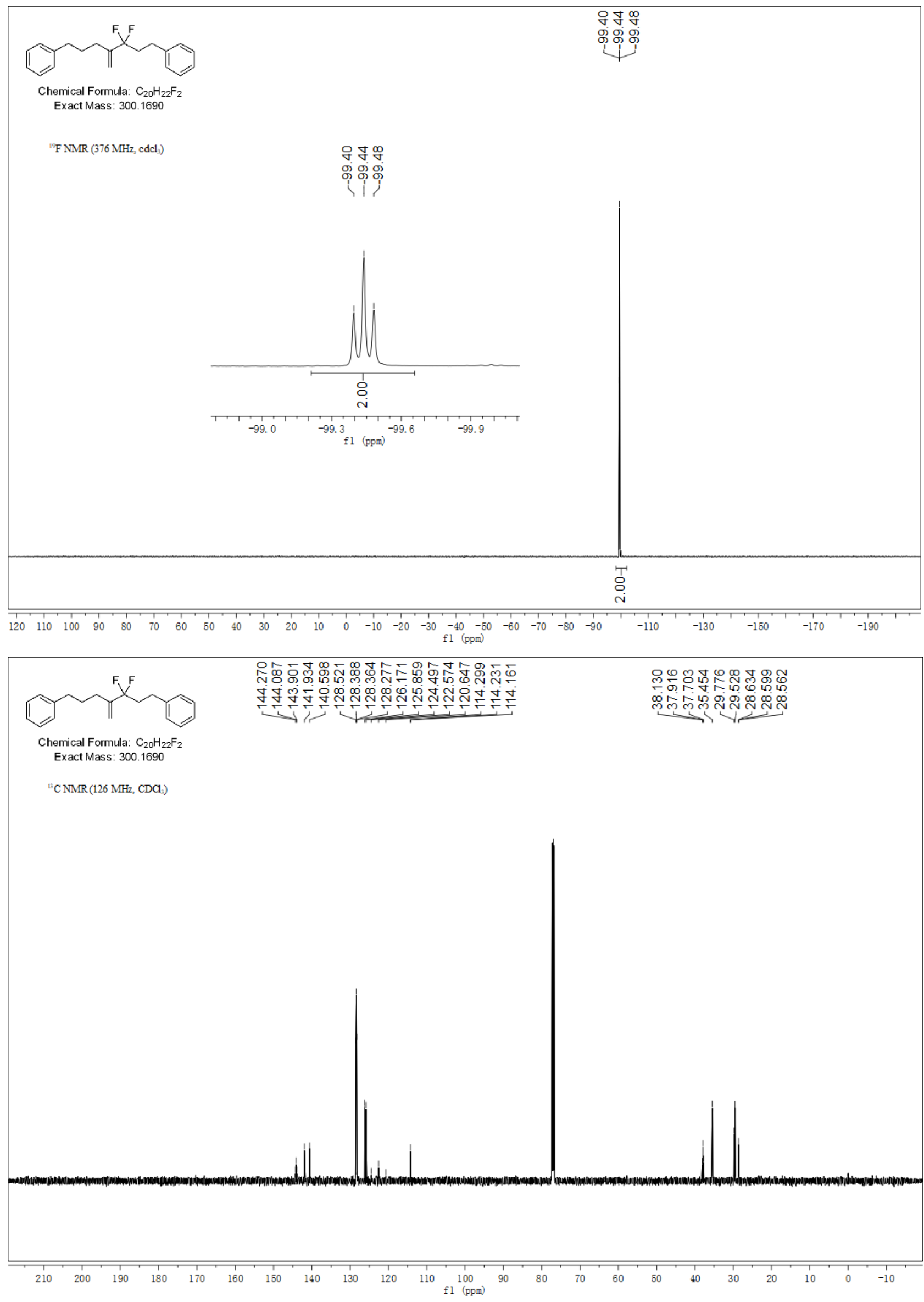
4-Ethynyl-3,3-difluoro-1,7-diphenylheptan-4-ol (14).
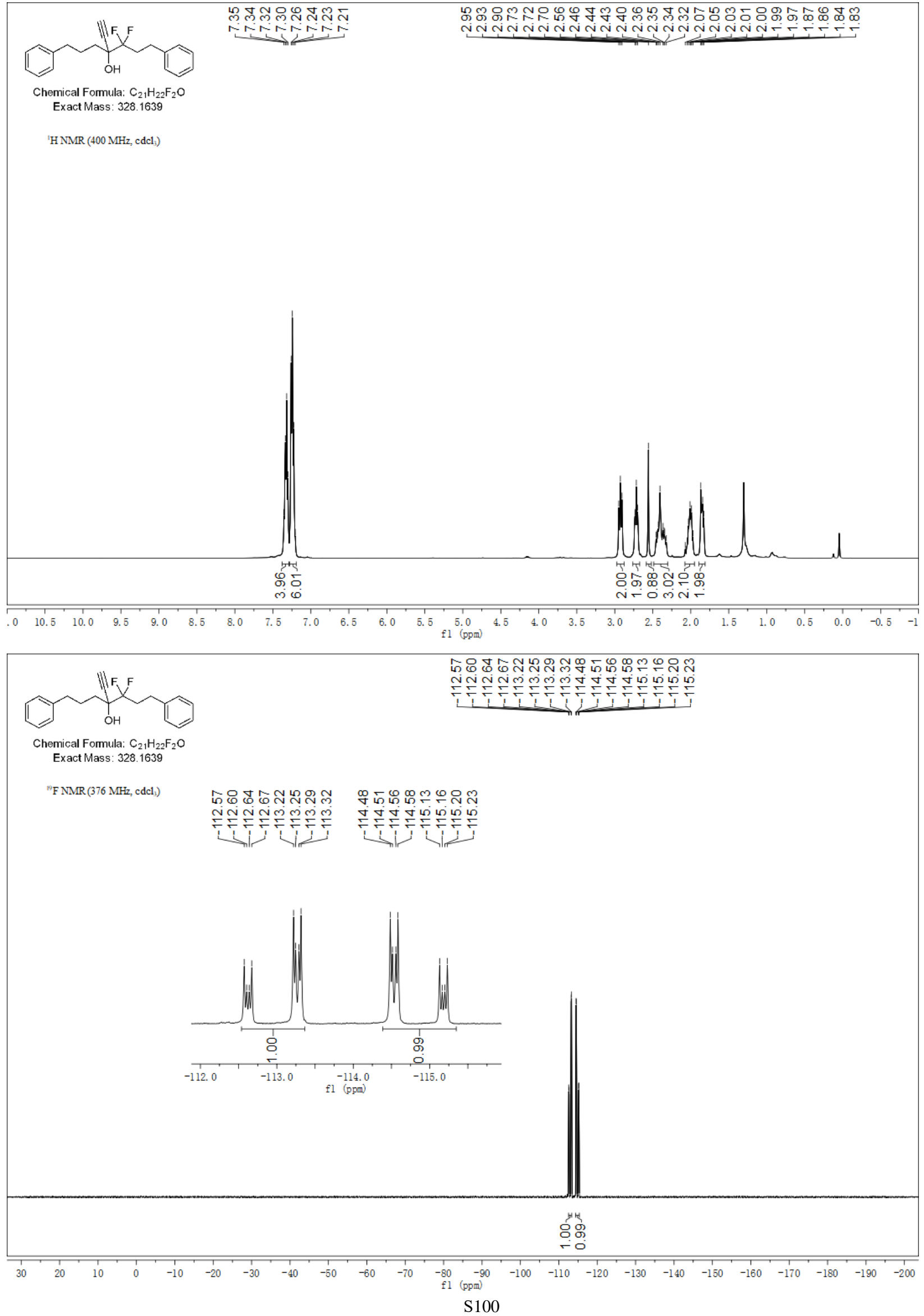


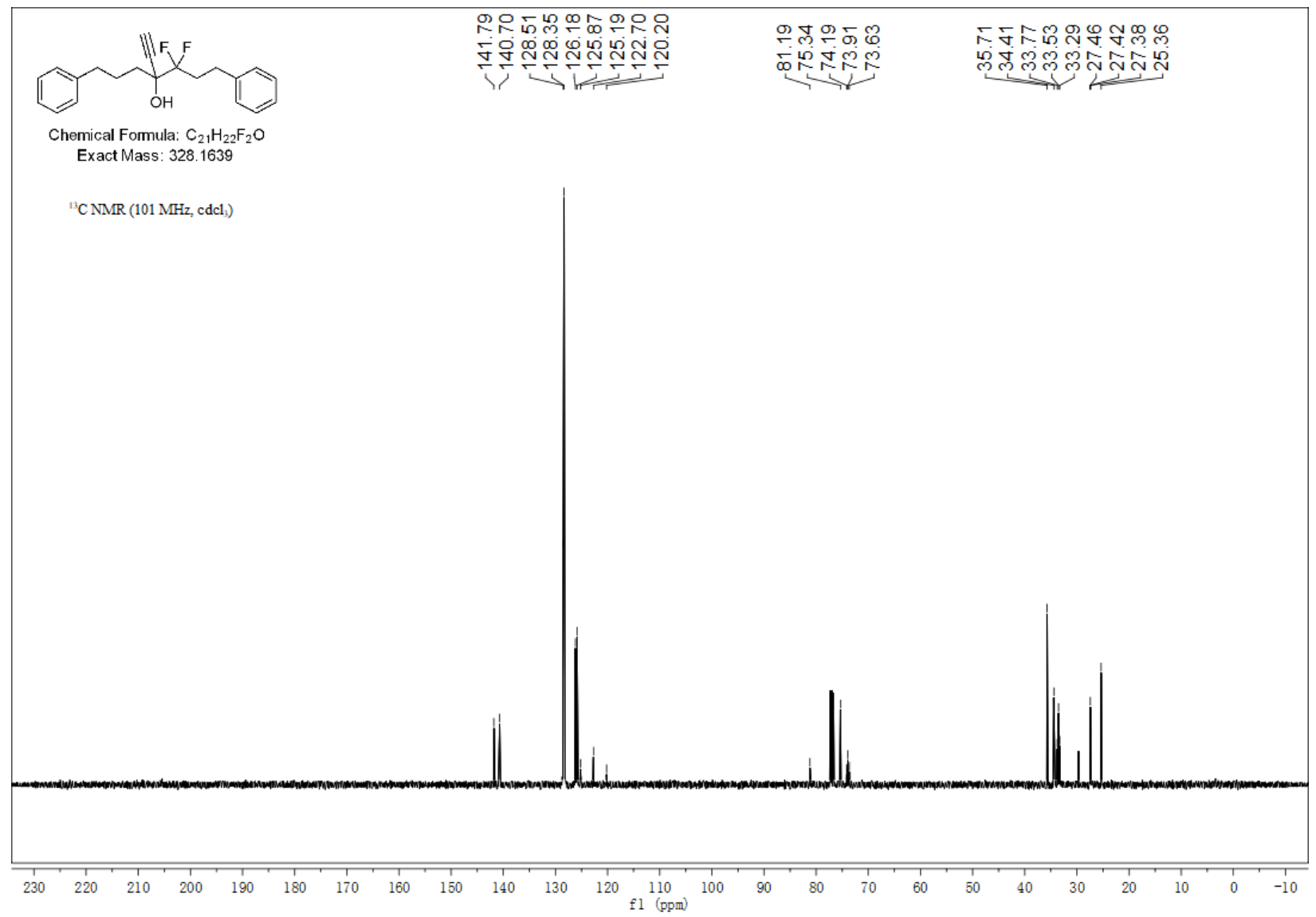

4-(2,2-Difluoro-4-phenylbutyl)-1,2-dihydronaphthalene (16).

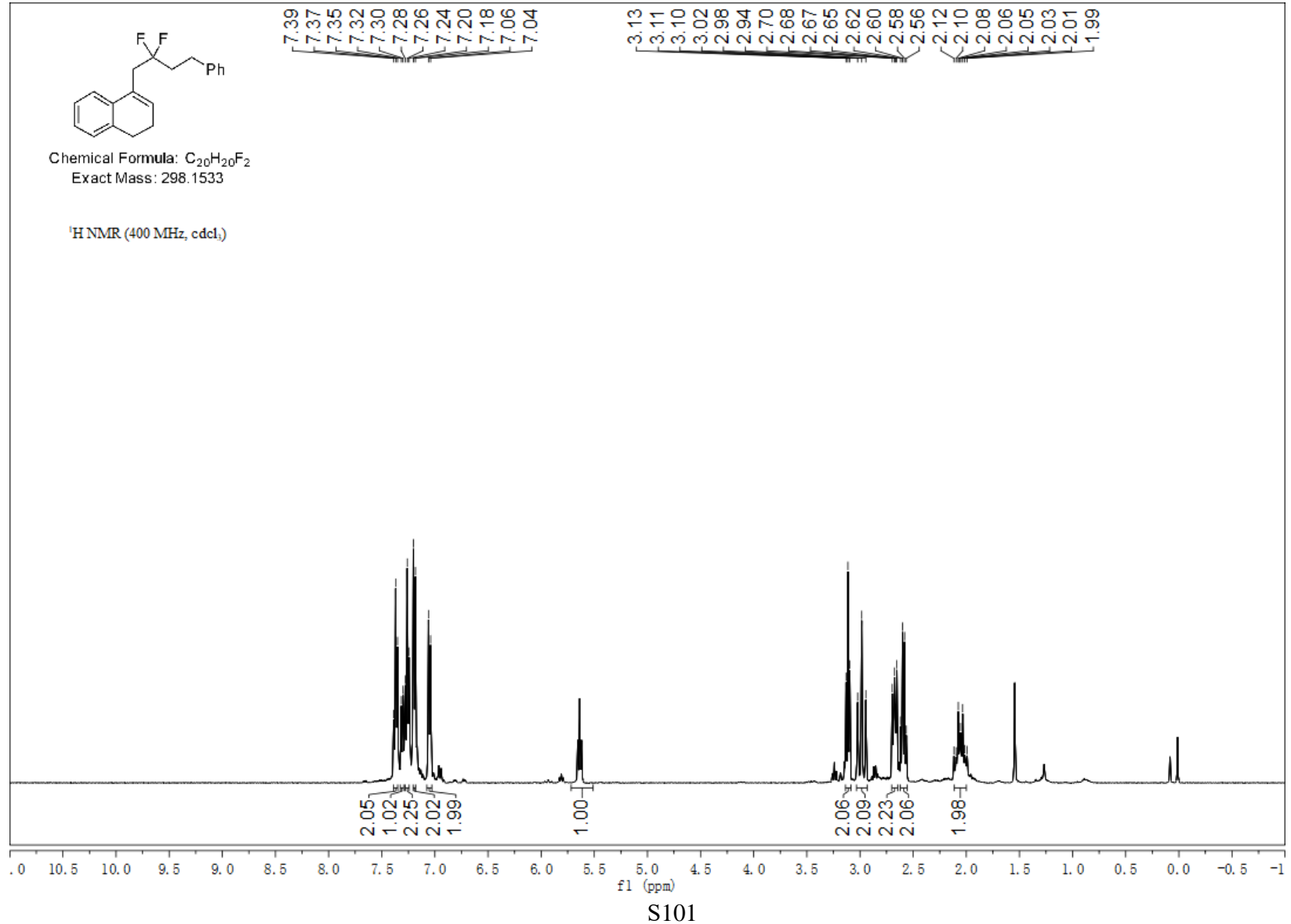



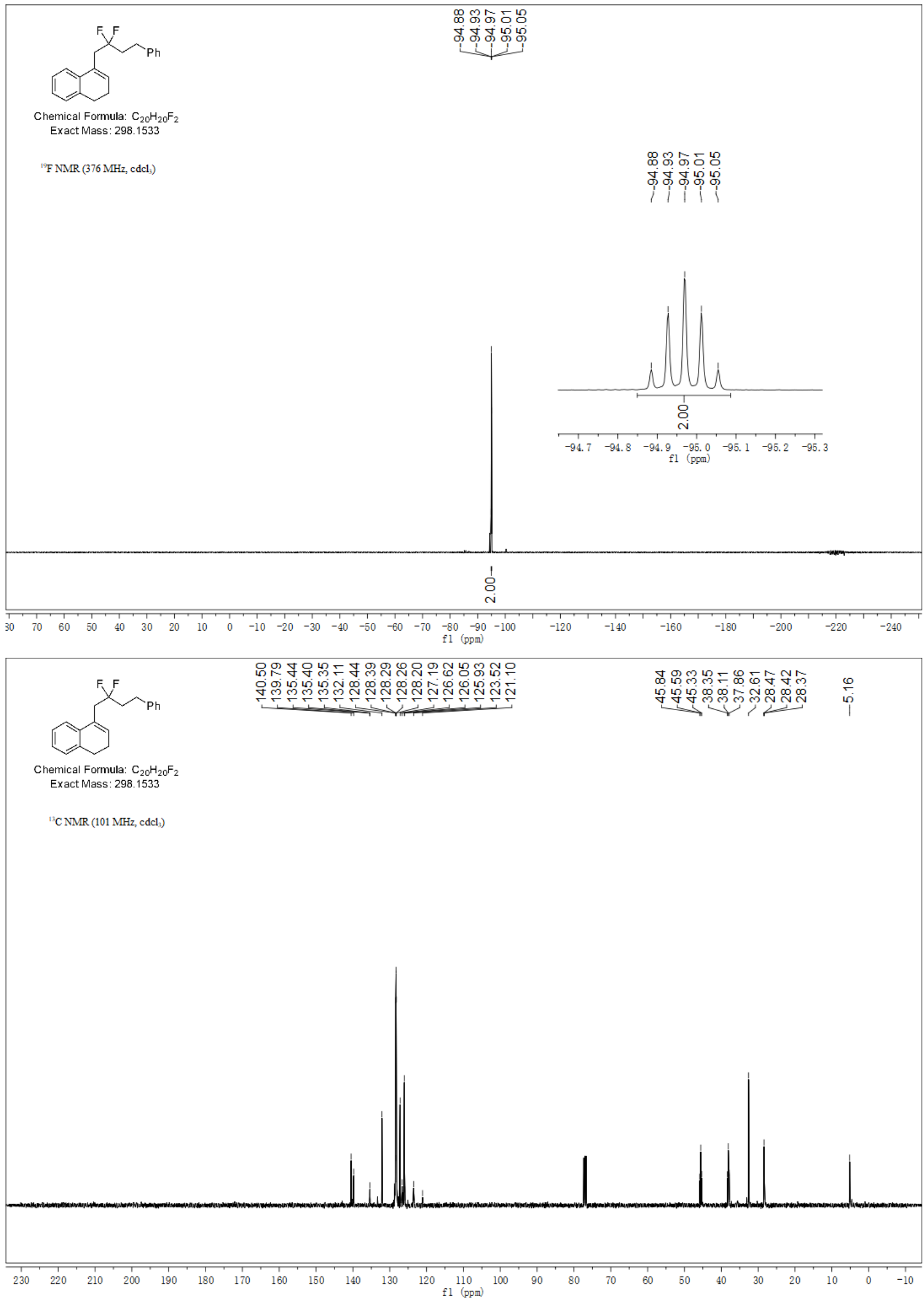